\title{
THE APPLICATION OF MODEL REDUCTION TECHNIQUES TO MEMS STRUCTURES
}

\author{
By \\ Marsha Bissessarsingh
}

\begin{abstract}
A thesis
presented to Carleton University in fulfilment of the

thesis requirement for the degree of MASTER OF APPLIED SCIENCE in ELECTRICAL ENGINEERING
\end{abstract}

Ottawa, Ontario, Canada

(C) Marsha C. Bissessarsingh, 2008 


$\begin{array}{ll}\begin{array}{l}\text { Library and } \\ \text { Archives Canada }\end{array} & \begin{array}{l}\text { Bibliothèque et } \\ \text { Archives Canada }\end{array} \\ \begin{array}{l}\text { Published Heritage } \\ \text { Branch }\end{array} & \begin{array}{l}\text { Direction du } \\ \text { Patrimoine de l'édition }\end{array} \\ \begin{array}{l}\text { 395 Wellington Street } \\ \text { Ottawa ON K1A 0N4 } \\ \text { Canada }\end{array} & \begin{array}{l}\text { 395, rue Wellington } \\ \text { Ottawa ON K1A 0N4 } \\ \text { Canada }\end{array}\end{array}$

Your file Votre référence ISBN: 978-0-494-40631-1 Our file Notre référence ISBN: 978-0-494-40631-1

NOTICE:

The author has granted a nonexclusive license allowing Library and Archives Canada to reproduce, publish, archive, preserve, conserve, communicate to the public by telecommunication or on the Internet, loan, distribute and sell theses worldwide, for commercial or noncommercial purposes, in microform, paper, electronic and/or any other formats.

The author retains copyright ownership and moral rights in this thesis. Neither the thesis nor substantial extracts from it may be printed or otherwise reproduced without the author's permission.
AVIS:

L'auteur a accordé une licence non exclusive permettant à la Bibliothèque et Archives Canada de reproduire, publier, archiver, sauvegarder, conserver, transmettre au public par télécommunication ou par l'Internet, prêter, distribuer et vendre des thèses partout dans le monde, à des fins commerciales ou autres, sur support microforme, papier, électronique et/ou autres formats.

L'auteur conserve la propriété du droit d'auteur et des droits moraux qui protège cette thèse. $\mathrm{Ni}$ la thèse ni des extraits substantiels de celle-ci ne doivent être imprimés ou autrement reproduits sans son autorisation.
In compliance with the Canadian Privacy Act some supporting forms may have been removed from this thesis.

While these forms may be included in the document page count, their removal does not represent any loss of content from the thesis.
Conformément à la loi canadienne sur la protection de la vie privée, quelques formulaires secondaires ont été enlevés de cette thèse.

Bien que ces formulaires aient inclus dans la pagination, il n'y aura aucun contenu manquant.

\section{Canada}




\section{Abstract}

Increased complexity of MEMS devices has emphasized the need for highly accurate mathematical representations. This is achieved through a system of second order differential equations and discretized through modeling techniques such as Finite Element Methods (FEM) and a tool based on beam theory, SUGAR. The ability to analyze these models through further mathematical manipulations is limited by the dimension of the resulting model size. Model reduction techniques based on Krylov subspace methods are a practical solution to achieving this, but face limitations in achieving stable responses as the parameters of the model are varied. An analysis and comparison of existing Krylov subspace methods is presented in addition to a reformulation of the techniques. This method proves to guarantee stability of reduced models and significantly reduce computational effort required. 


\section{Acknowledgements}

I would like to sincerely thank Professor Tom Smy who has gone above and beyond the role of a supervisor in so many ways. His unwavering patience, support and encouragement through many trying times has been incomparable.

I would also like to thank so many of my friends for listening, caring and guiding me to the light at the end of the tunnel. In addition, I would also like to mention all the professors and support staff in the Department of Electronics whose sense of humor has made my time at Carleton fun and interesting. 
To my fish, Charlie. We did it buddy. 


\section{Table of Contents}

$\begin{array}{ll}\text { Abstract } & \text { ii }\end{array}$

$\begin{array}{ll}\text { Acknowledgements } & \text { iii }\end{array}$

Table of Contents $\quad$ v

List of Figures $\quad$ x

List of Acronyms $\quad$ xvi

List of Symbols $\quad$ xvii

Definition of Terms $\quad$ xx

1 Introduction 1

1.1 Background and Motivation $\ldots \ldots \ldots \ldots \ldots$

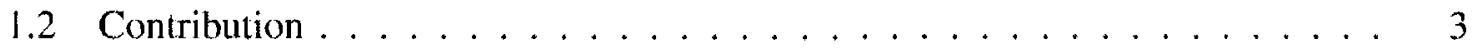

1.3 Organization of Thesis ......................... 4

2 Micro-electromechanical Systems and Modeling Techniques 6

2.1 Micro-electromechanical Systems . . . . . . . . . . . . . . 6

2.2 Finite Element Modeling . . . . . . . . . . . . . . . . . . . . . 9

2.2 .1 Basic FEM theory . . . . . . . . . . . . . . . 11

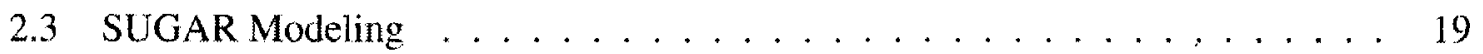

2.3.1 Overview of SUGAR Modeling Theory and Implementation . . . . . 20

2.4 Analysis of MEMS . . . . . . . . . . . . . . . . . . . . . . . . . . . . . . . . .

2.4.1 Frequency Domain Analysis . . . . . . . . . . . . . . . 25

2.4 .2 Time Domain Analysis . . . . . . . . . . . . . . 26

2.4 .3 Damping in MEMS . . . . . . . . . . . . . . . . . 27

System Behavior ........................ 28 
3 Review of Model Reduction Techniques

3.1 Model Reduction of Second Order Systems . . . . . . . . . . . . . . . 31

3.2 Moments of a System . . . . . . . . . . . . . . . . . . 33

3.3 Concepts of Direct Moment Matching Techniques . . . . . . . . . . . . . . . 34

3.4 Indirect Moment Matching Techniques . . . . . . . . . . . . . . . . 35

3.5 Krylov subspaces . . . . . . . . . . . . . . . . . . . . . . . . . . . . . . . . . .

3.6 The Arnoldi Algorithm . . . . . . . . . . . . . . . . . . . 37

3.7 Model Reduction of Second Order Systems via Arnoldi . . . . . . . . . . . . . 41

3.8 The Second Order Arnoldi (SOAR) Algorithm . . . . . . . . . . . . . . . . . . 44

3.9 Model Reduction of Second Order Systems via SOAR . . . . . . . . . . . 47

3.10 Conclusion . . . . . . . . . . . . . . . . . . 51

4 Stability and Passivity of Model Reduction $\quad \mathbf{5 3}$

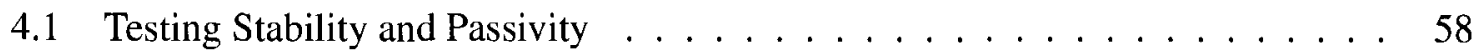

4.1 .1 Results and Conclusions . . . . . . . . . . . . . . . . 61

5 Frequency Response of Krylov Based Model Reduction Techniques 64

5.1 Error Analysis . . . . . . . . . . . . . . . . . . . . . . . . . . . . . . 64

5.2 FEM Cantilever Model . . . . . . . . . . . . . . . . . . . 65

5.2.1 Cantilever Models with Damping $=0 \times M \ldots \ldots 65$

540-node Cantilever . . . . . . . . . . . . . . 65

2700-node Cantilever . . . . . . . . . . . . . . 68

5.2.2 Cantilever Models with Damping $=10^{-2} \times M \ldots \ldots 71$

540 -node Cantilever . . . . . . . . . . . . . . . . 71

2700-node Cantilever . . . . . . . . . . . . . . . . 73

5.2.3 Cantilever Models with Damping $=10^{-1} \times M \ldots \ldots 75$

540-node Cantilever . . . . . . . . . . . . . . . . 75

2700-node Cantilever . . . . . . . . . . . . . . . . 77

5.2.4 Conclusion on Frequency Response of FEM Models . . . . . . . . . . 79

5.3 SUGAR Models . . . . . . . . . . . . . . . . . . . . . . 80

5.3.1 SUGAR Models and the Arnoldi Algorithm . . . . . . . . . . . . . 80

150-element SUGAR model . . . . . . . . . . . . . . . . . . . . 80

500 -element SUGAR model . . . . . . . . . . . . . . . 81

5.3.2 SUGAR Models and the SOAR Procedure . . . . . . . . . . . . . 85

150-element SUGAR model . . . . . . . . . . . . . . . 85

500-element SUGAR Model . . . . . . . . . . . . . . . . . . . . 88

5.3.3 Conclusion on Frequency Response of SUGAR Models . . . . . . . 90

5.4 Conclusion . . . . . . . . . . . . . . . . . . . . . 90

6 Time Domain Analysis of Krylov Based Model Reduction Techniques 92

6.1 Error Analysis . . . . . . . . . . . . . . . . . . . . . . . . . . . . . . . . . . .

6.2 FEM Cantilever Model . . . . . . . . . . . . . . . . . . . 94 
6.2.1 Cantilever Models with Damping $=0 \times M \ldots \ldots 95$

Small Cantilever Built with 540 nodes . . . . . . . . . . . . . 95

Large Cantilever Built with 2700 nodes . . . . . . . . . . . . . . . 99

6.2.2 Cantilever Models with Damping=0.01 $\times M \ldots \ldots$. . . . . . . . 102

Small Cantilever Built with 540-nodes . . . . . . . . . . . . . 102

Large Cantilever Built with 2700-nodes . . . . . . . . . . . . . . . 105

6.2.3 Cantilever Models with Damping $=10^{-1} \times M \ldots . . . . . . . . .107$

Small Cantilever Built with 540 nodes . . . . . . . . . . . . . . 107

Large Cantilever Built with 2700-nodes . . . . . . . . . . . . . . 110

6.2.4 Conclusion on the Time Domain Analysis of FEM models . . . . . . . 110

6.3 SUGAR Models . . . . . . . . . . . . . . . . . . . . . 111

6.3.1 Numerical Analysis of SUGAR Models . . . . . . . . . . . . . . . 111

Conclusion on the Time Domain Analysis of SUGAR Models . . . . . 117

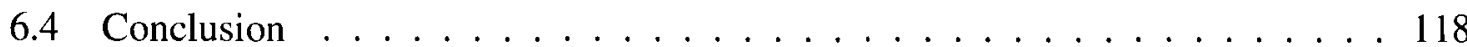

7 The Impact of Damping on Model Reduction 121

7.1 Motivation . . . . . . . . . . . . . . . . 121

7.2 The Contribution of Damping . . . . . . . . . . . . . . . 122

7.2.1 Moments of a Second Order System . . . . . . . . . . . . . . . . 123

Summary of Results of Moment Analysis: Analytical and Numerical . 125

7.2.2 The Effect of Damping on the orthogonal $Q$-matrix . . . . . . . . . 126

7.2.3 Effect of Damping on the Stability of Arnoldi and SOAR . . . . . . . . 128

7.2.4 Summary on the Effects of Damping on the Stability of Reduced Models 133

7.3 Undamped Krylov Subspace Methods and Second-Order Systems . . . . . . 134

7.3.1 The Undamped SOAR Procedure . . . . . . . . . . . . . . . . . . 136

7.3.2 The Undamped Arnoldi Algorithm . . . . . . . . . . . . . . . . 136

7.3.3 Numerical Analysis of Undamped Krylov Subspace Methods . . . . . 137

Stability Results . . . . . . . . . . . . . . . . . . . . 138

7.3 .4 Time Domain Analysis . . . . . . . . . . . . . . . . . . 141

7.3.5 Numerical Analysis of Complex Structures . . . . . . . . . . . . . . 144

7.3.6 Serpentine ... . . . . . . . . . . . . . . . . . 144

Response of the Serpentine Structure . . . . . . . . . . . . 145

7.3.7 H-shape Resonator . . . . . . . . . . . . . . . . . . . . . . . . 147

Response of the H-Shape Resonator . . . . . . . . . . . . . . . . 148

7.3 .8 Elevated Inductor . . . . . . . . . . . . . . . . . . . 150

Response of the Elevated Inductor . . . . . . . . . . . . . . 151

7.3.9 SUGAR Models and SOAR . . . . . . . . . . . . . . . 154

7.4 Conclusion . . . . . . . . . . . . . . . . . 157

8 Conclusion $\quad 159$

$\begin{array}{lll}\text { Appendix A } & \text { Moment analysis } & 164\end{array}$ 
Appendix C Frequency Response of the Cantilever Beam $<10^{-2} \quad 174$

C.1 1350-node Cantilever Model with Damping $=0.0 \times M \ldots \ldots \ldots \ldots 174$

C.2 1350-node Cantilever Model with Damping $=10^{-2} \times M \ldots \ldots \ldots$

C.2.1 1350-node Cantilever Model with Damping $=10^{-1} \times M \ldots \ldots . .179$

1350-node Cantilever . . . . . . . . . . . . . . . . . . . 179

C.3 Cantilever Models with Damping $=1.0 \times M \ldots \ldots \ldots \ldots 18 \ldots \ldots$

C.3.1 540-node Cantilever . . . . . . . . . . . . . . . . . 181

C.3.2 1350-node Cantilever . . . . . . . . . . . . . . . . . . 183

C.3.3 2700-node Cantilever . . . . . . . . . . . . . . . . . 185

C.4 Cantilever Models with Damping $=10^{-3} \times M \ldots \ldots \ldots \ldots$. . . . 187

540-node Cantilever . . . . . . . . . . . . . . . . . . . . 187

C.4.1 1350-node Cantilever . . . . . . . . . . . . . . . . . . . . . 189

C.4.2 2700-node Cantilever . . . . . . . . . . . . . . . . . . . . 191

C.5 Cantilever Models with Damping $=10^{-4} \times M \ldots \ldots \ldots$. . . . . 193

540-node Cantilever . . . . . . . . . . . . . . . . 193

C.5.1 1350-node Cantilever . . . . . . . . . . . . . . . . . 195

C.5.2 2700-node Cantilever . . . . . . . . . . . . . . . . . 197

C.6 Cantilever Models with Damping $=10^{-5} \times M \ldots \ldots \ldots \ldots$. . . . 199

C.6.1 540-node Cantilever . . . . . . . . . . . . . . . . . . . . . 199

C.6.2 1350-node Cantilever . . . . . . . . . . . . . . . . . . . 201

C.6.3 2700-node Cantilever . . . . . . . . . . . . . . . . . . 203

Appendix D Additional Time Domain Response for the FEM Cantilever Model 206

D.1 FEM Cantilever Model with Damping $=0 \times M \ldots \ldots \ldots \ldots$

Medium Cantilever built with 1350 tetrahedrons . . . . . . . . 206

D.2 FEM Cantilever Model with Damping $=10^{-2} \times M \ldots \ldots \ldots \ldots$

Medium Cantilever built with 1350-nodes . . . . . . . . . . . 210

D.3 FEM Cantilever Model with Damping $=10^{-1} \times M \ldots \ldots \ldots \ldots . \ldots 213$

Medium Cantilever Built with 1350 tetrahedrons . . . . . . . . . 213

D.4 FEM Cantilever Model with Damping $=1 \times M \ldots \ldots \ldots \ldots \ldots$

D.4.1 Small Cantilever Built with 540 tetrahedrons . . . . . . . . . 216

D.4.2 Medium Cantilever Built with 1350 tetrahedrons . . . . . . . . 219

D.4.3 Large Cantilever Built with 2700 tetrahedrons . . . . . . . . . . . 222

D.5 Cantilever Model with Damping $\leq D=10^{-3} \times M \ldots \ldots \ldots$

D.5.1 Small Cantilever Built with 540-nodes . . . . . . . . . . . . . . 224

D.5.2 Medium Cantilever Built with 1350-nodes . . . . . . . . . . . 231

D.5.3 Large Cantilever Built with 2700-nodes . . . . . . . . . . . 237

$\begin{array}{lll}\text { Appendix E } & \text { Further Eigenvalue Numerical Results } & 243\end{array}$ 


\section{List of Figures}

2.1 Micro-mirror MEMS system . . . . . . . . . . . . . . . . 7

2.2 Modeled MEMS Structures . . . . . . . . . . . . . . . . . . 15

2.3 One Block decomposed into Tetrahedron Elements . . . . . . . . . . . 16

2.4 The Linear Tetrahedron Element . . . . . . . . . . . . . . . . . . . . 16

2.5 Linear Tetrahedron Elements Assembled into a Cantilever . . . . . . . . . . . 17

2.6 Sparsity of MEMS Representative matrices modeled using FEM . . . . . . . 18

2.7 Beam Element used in SUGAR . . . . . . . . . . . . . . . . . . . . . . . . . . . . . .

2.8 Cantilever created with 6 SUGAR Elements . . . . . . . . . . . . . . . 23

2.9 Sparsity of MEMS Representative matrices modeled using SUGAR . . . . . . 24

2.10 Damping Response of an Analytical Cantilever . . . . . . . . . . . . . . . 29

3.1 The Arnoldi Algorithm . . . . . . . . . . . . . . . . . . . . . . . . . 40

3.2 The SOAR procedure . . . . . . . . . . . . . . . 47

5.1 Comparison of frequency response for the full system and reduced model of a 540 node Cantilever with Damping=0 $\times M \ldots \ldots \ldots 6$

5.2 Frequency Response . . . . . . . . . . . . . . . . . . . . 67

5.3 Comparison of frequency response for the full system and reduced model of a 2700 node Cantilever with Damping=0 $\times M \ldots \ldots$. . . . . . . 68

5.4 Frequency Response . . . . . . . . . . . . . . . . . . . . . . . . . 69

5.5 Comparison of frequency response for the full system and reduced model of a 540 node Cantilever with Damping $=10^{-2} \times M \ldots \ldots . \ldots 71$

5.6 Frequency Response . . . . . . . . . . . . . . . . . . . . . 72

5.7 Comparison of frequency responses for the full system and reduced model of a 2700-node Cantilever with Damping $=10^{-2} \times M \ldots \ldots 73$

5.8 Frequency Response . . . . . . . . . . . . . . . . . . . . . . 74

5.9 Comparison of frequency response for the full system and reduced model of a 540 node Cantilever with Damping $=10^{-1} \times M \ldots \ldots . \ldots 75$

5.10 Frequency Response . . . . . . . . . . . . . . . . . . . . 76

5.11 Comparison of frequency response for the full system and reduced model of a 2700 node Cantilever with Damping $=10^{-1} \times M \ldots \ldots 77$

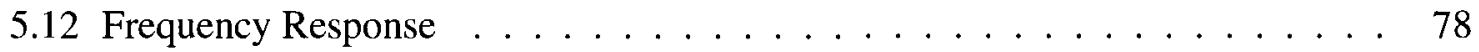


5.13 Frequency Response for a SUGAR Model with 150 elements using Arnoldi .

5.14 Relative Percentage Error of the Frequency Domain Response for a SUGAR Model with 150 elements using Arnoldi . . . . . . . . . . . . . . . 82

5.15 Frequency Response for a SUGAR Model with 500 elements using Arnoldi . . 83

5.16 Relative Percentage Error of the Frequency Domain Response for a SUGAR Model with 500 elements using Arnoldi . . . . . . . . . . . . . . . . . 84

5.17 Frequency Response for a SUGAR Model with 150 elements using SOAR . . . 86

5.18 Relative Percentage Error of the Frequency Domain Response for a SUGAR Model with 150 elements using SOAR . . . . . . . . . . . . 87

5.19 Frequency Response for a SUGAR Model with 500 elements using SOAR . . . 88

5.20 Relative Percentage Error of the Frequency Domain Response for a SUGAR Model with 500 elements using SOAR . . . . . . . . . . . . .

6.1 Comparison of time domain response for the full system and reduced models of a 540-node Cantilever with Damping=0 $\times M \ldots \ldots$. . . . . . . . 96

6.2 SOAR Cantilever Results . . . . . . . . . . . . . . . . . . . . . . . 98

6.3 Comparison of time domain response for the full system and reduced models of a 2700 -node Cantilever with Damping=0 $\times M \ldots \ldots$. . . . . . . . . 99

6.4 SOAR Cantilever Results . . . . . . . . . . . . . . . . . . . . 101

6.5 Comparison of time domain response for the full system and reduced models of

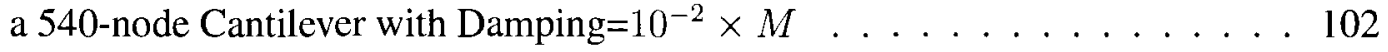

6.6 SOAR Cantilever Results . . . . . . . . . . . . . . . . . . . . . . . 104

6.7 Comparison of time domain response for the full system and reduced models of a 2700 -node Cantilever with Damping $=10^{-2} \times M \ldots \ldots . . \ldots 105$

6.8 SOAR Cantilever Results . . . . . . . . . . . . . . . . . . . . . 106

6.9 Comparison of time domain response for the full system and reduced systems of a 540-node Cantilever with Damping $=10^{-1} \times M \ldots \ldots 107$

6.10 SOAR Cantilever Results . . . . . . . . . . . . . . . . . . . . . . . 109

6.11 Comparison of time domain response for the full system and SOAR reduced model of a 2700-node Cantilever with Damping $=10^{-1} \times M$, with 10 moments . 110

6.12 Time Domain Response for a SUGAR Model with 150-elements . . . . . . . . 113

6.13 Time Domain Response for a SUGAR Model with 500-elements . . . . . . . . 114

6.14 Relative Error of the Time Domain Response for a SUGAR Model with 150elements . . . . . . . . . . . . . . . . . . 115

6.15 Relative Error of the Time Domain Response for a SUGAR Model with 500elements ........................... 116

7.1 Rank Results . . . . . . . . . . . . . . . . . . . . . . . . 127

7.2 Eigenvalues of Arnoldi Reduced Models with a Range in Damping, 540-node reduced model with 30 moments . . . . . . . . . . . . . . . . 130

7.3 Eigenvalues of SOAR Reduced Models with a Range in Damping, 540-node reduced model with 30 moments . . . . . . . . . . . . . . . . 132 
7.4 Relative Errors of Reduced Models using Rayleigh Damping . . . . . . . . . . 135

7.5 Eigenvalues of Reduced Models using Rayleigh Damping, $\alpha=1$. . . . . . . . 139

7.6 Eigenvalues of Reduced Models using Rayleigh Damping, $\alpha=10$. . . . . . . 140

7.7 Time domain Response Comparison of Reduced Models and Full model using Rayleigh Damping . . . . . . . . . . . . . . . . . . . . . . . 142

7.8 Relative Errors of the TD Simulation for Reduced Models using Rayleigh Damping . . . . . . . . . . . . . . . . . . . . . 143

7.9 Serpentine structure used to verify $\mathrm{Q}$ formed with $\mathrm{D}=0 \ldots \ldots \ldots$

7.10 Serpentine structure used to verify $Q$ formed with $D=0$ - Overdamped . . . . . 145

7.11 Serpentine structure used to verify $Q$ formed with $D=0$ - Approaching Critical . 145

7.12 Serpentine structure used to verify $Q$ formed with $D=0$ - Underdamped . . . . . 146

7.13 Serpentine structure used to verify $Q$ formed with $D=0$ - No Damping . . . . . 147

$7.14 \mathrm{H}$-shape Resonator structure used to verify $\mathrm{Q}$ formed with $\mathrm{D}=0$. . . . . . . . . 148

$7.15 \mathrm{H}$-shape Resonator used to verify $\mathrm{Q}$ formed with $\mathrm{D}=0$ - Overdamped . . . . . 148

7.16 H-shape Resonator used to verify q0 - Approaching Critical . . . . . . . . . . . 149

7.17 H-shape Resonator used to verify Q formed with $\mathrm{D}=0$ - Underdamped . . . . . 149

7.18 H-shape Resonator used to verify $Q$ formed with $D=0$ - No Damping . . . . . . 150

7.19 Elevated Inductor structure used to verify $Q$ formed with $D=0 \ldots \ldots$. . . . . 151

7.20 Elevated Inductor used to verify $Q$ formed with $D=0$ - Overdamped . . . . . 152

7.21 Elevated Inductor used to verify $Q$ formed with $\mathrm{D}=0$ - Approaching Critical . . 152

7.22 Elevated Inductor used to verify $Q$ formed with $D=0$ - Underdamped . . . . . . 153

7.23 Elevated Inductor used to verify $Q$ formed with $\mathrm{D}=0$ - No Damping . . . . . 153

7.24 Relative error of 150-element SUGAR Model used to verify Q formed with $\mathrm{D}=0$, with SOAR . . . . . . . . . . . . . . . . . 155

7.25 Relative error of 150-element SUGAR Model used to verify Q formed with $\mathrm{D}=0$, with SOAR . . . . . . . . . . . . . . . . . 155

7.26 Time domain response of a 150-element SUGAR Model used to verify $Q$ formed with $\mathrm{D}=0$, all 16 companion forms, . . . . . . . . . . . . 156

7.27 Relative error of 150-element SUGAR Model used to verify $\mathrm{Q}$ formed with $\mathrm{D}=0$, with Arnoldi . . . . . . . . . . . . . . . . . . . . 157

C.1 Comparison of frequency response for the full system and reduced model of a 1350 node Cantilever with Damping=0 $\times$. . . . . . . . . . . . . 175

C.2 Frequency Response . . . . . . . . . . . . . . . . . . . 176

C.3 Comparison of frequency responses for the full system and reduced model of a

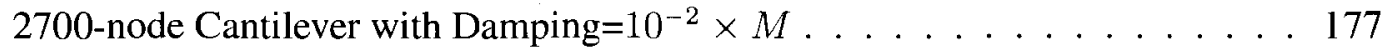

C.4 Frequency Response . . . . . . . . . . . . . . . . . . . . 178

C.5 Comparison of frequency response for the full system and reduced model of a

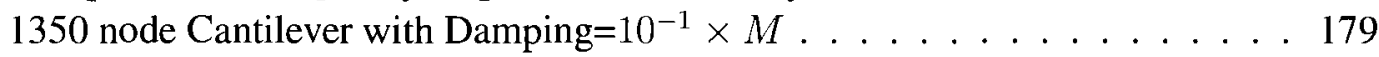

C.6 Frequency Response . . . . . . . . . . . . . . . . . . . . . . . . 180

C.7 Comparison of frequency responses for the full system and reduced model of a 540 node Cantilever with Damping=1.0 $\times M \ldots \ldots$. . . . . . . 181 
C.8 Frequency Response . . . . . . . . . . . . . . . . . . . . . . . . . . . . . . . 182

C.9 Comparison of frequency response for the full system and reduced model of a 1350 node Cantilever with Damping $=1.0 \times M \ldots \ldots \ldots$. . . . . . 183

C.10 Frequency Response . . . . . . . . . . . . . . . . . . . . . . . . . . . 184

C.11 Comparison of frequency response for the full system and reduced model of a 2700 node Cantilever with Damping $=1.0 \times M \ldots \ldots \ldots$. . . . . . 185

C.12 Frequency Response . . . . . . . . . . . . . . . . . . . . . . . 186

C.13 Comparison of frequency responses for the full system and reduced model of a 540-node Cantilever with Damping $=10^{-3} \times M \ldots \ldots \ldots$. . . . 187

C.14 Frequency Response . . . . . . . . . . . . . . . . . . . . . . 188

C.15 Comparison of frequency responses for the full system and reduced model of a 1350-node Cantilever with Damping $=10^{-3} \times M \ldots \ldots \ldots$. . . . . 189

C.16 Frequency Response . . . . . . . . . . . . . . . . . . . . . . . . . . . . 190

C.17 Comparison of frequency responses for the full system and reduced model of a 2700-node Cantilever with Damping $=10^{-3} \times M \ldots \ldots$. . . . . . . 191

C.18 Frequency Response . . . . . . . . . . . . . . . . . . . . . . . . . 192

C.19 Comparison of frequency responses for the full system and reduced model of a 540-node Cantilever with Damping $=10^{-4} \times M \ldots \ldots \ldots$

C.20 Frequency Response . . . . . . . . . . . . . . . . . . . . . . . . . . 194

C.21 Comparison of frequency responses for the full system and reduced model of a 1350-node Cantilever with Damping $=10^{-4} \times M \ldots \ldots \ldots \ldots$

C.22 Frequency Response . . . . . . . . . . . . . . . . . . . . . . . . 196

C.23 Comparison of frequency responses for the full system and reduced model of a 2700-node Cantilever with Damping $=10^{-4} \times M \ldots \ldots$. . . . . . . 197

C.24 Frequency Response . . . . . . . . . . . . . . . . . . . . . . . . . . . 198

C.25 Comparison of frequency responses for the full system and reduced model of a 540-node Cantilever with Damping $=10^{-5} \times M \ldots \ldots . . . \ldots . .199$

C.26 Frequency Response . . . . . . . . . . . . . . . . . . . . . . . 200

C.27 Comparison of frequency responses for the full system and reduced model of a 1350-node Cantilever with Damping $=10^{-5} \times M \ldots \ldots \ldots \ldots 20 \ldots$

C.28 Frequency Response . . . . . . . . . . . . . . . . . . . . . . . . 202

C.29 Comparison of frequency responses for the full system and reduced model of a 2700-node Cantilever with Damping $=10^{-5} \times M \ldots \ldots \ldots \ldots$

C.30 Frequency Response . . . . . . . . . . . . . . . . . . . . . . 204

D.1 Comparison of time domain response for the full system and Arnoldi reduced model of a 1350-node Cantilever with Damping=0 $\times M \ldots \ldots$. . . . . 207

D.2 Comparison of time domain response for the full system and SOAR reduced model of a 1350-node Cantilever with Damping $=0 \times M \ldots \ldots 207$

D.3 SOAR Cantilever Results . . . . . . . . . . . . . . . . . . . . . . . . 209

D.4 Comparison of time domain response for the full system and Arnoldi reduced model of a 1350-node Cantilever with Damping $=10^{-2} \times M$, with 30 moments . 210 
D.5 Comparison of time domain response for the full system and SOAR reduced model of a 1350-node Cantilever with Damping $=10^{-2} \times M$, with 10 moments . 210

D.6 SOAR Cantilever Results . . . . . . . . . . . . . . . . . . 212

D.7 Comparison of time domain response for the full system and Arnoldi reduced models of a 1350-node Cantilever with Damping=0.1 $\times M$, with 70 moments . 213

D.8 Comparison of time domain response for the full system and SOAR reduced model of a 1350-node Cantilever with Damping=0.1 $\times M$, with 10 moments . 213

D.9 SOAR Cantilever Results . . . . . . . . . . . . . . . . . . 215

D.10 Comparison of time domain response for the full system and SOAR reduced model of a 540-node Cantilever with Damping=1 $\times M$, with 10 moments . . 216

D.11 SOAR Cantilever Results . . . . . . . . . . . . . . . . . . 218

D.12 Comparison of time domain response for the full system and Arnoldi reduced model of a 1350-node Cantilever with Damping $=1 \times M$, with 90 and 110 moments 219

D.13 Comparison of time domain response for the full system and SOAR reduced model of a 1350-node Cantilever with Damping=1*M, with 10 moments . . . . 219

D.14 SOAR Cantilever Results . . . . . . . . . . . . . . . . . . . . 221

D.15 Comparison of time domain response for the full system and SOAR reduced model of a 2700-node Cantilever with Damping=1 $\times M$, with 10 moments . . 222

D.16 SOAR Cantilever Results . . . . . . . . . . . . . . . . . . . . . 223

D.17 Comparison of time domain Response for the full system and Arnoldi reduced models of a 540-node Cantilever with Damping $=10^{-3} \times M, 10^{-4} \times M$ and $10^{-5} \times M$, with 10 moments . . . . . . . . . . . . . . . 225

D.18 Comparison of time domain response for the full system and SOAR reduced model of a 540-node Cantilever with Damping $=10^{-3} \times M, 10^{-4} \times M$ and $10^{-5} \times$ $M$, with 10 moments . . . . . . . . . . . . . . . 226

D.19 SOAR Cantilever Results . . . . . . . . . . . . . . . . . . . . . 228

D.20 SOAR Cantilever Results . . . . . . . . . . . . . . . . . . . . . . . . . . . . . . . . . . . . . . . . . . . . . . . . . . . . .

D.21 SOAR Cantilever Results . . . . . . . . . . . . . . . . . 230

D.22 Comparison of time domain Response for the full system and Arnoldi reduced models of a 1350-node Cantilever with Damping $=10^{-3} \times M, 10^{-4} \times M$ and $10^{-5} \times M$, with 10 moments . . . . . . . . . . . . . 231

D.23 Comparison of time domain response for the full system and SOAR reduced model of a 1350-node Cantilever with Damping $=10^{-3} \times M, 10^{-4} \times M$ and $10^{-5} \times M$, with 10 moments . . . . . . . . . . . . . 232

D.24 SOAR Cantilever Results . . . . . . . . . . . . . . . . . . . 234

D.25 SOAR Cantilever Results . . . . . . . . . . . . . . . . . . . . . . . . . . . . . . . . . . . . . . . . . .

D.26 SOAR Cantilever Results . . . . . . . . . . . . . . . . . . . 236

D.27 Comparison of time domain Response for the full system and Arnoldi reduced models of a 2700-node Cantilever with Damping $=10^{-3} \times M, 10^{-4} \times M$ and $10^{-5} \times M$, with 10 moments . . . . . . . . . . . . . . 237 
D.28 Comparison of time domain response for the full system and SOAR reduced model of a 2700 -node Cantilever with Damping $=10^{-3} \times M, 10^{-4} \times M$ and $10^{-5} \times M$, with 10 moments . . . . . . . . . . . . . . . . 238

D.29 SOAR Cantilever Results . . . . . . . . . . . . . . . . . . . . . . . 240

D.30 SOAR Cantilever Results . . . . . . . . . . . . . . . . . . . . . . 241

D.31 SOAR Cantilever Results . . . . . . . . . . . . . . . . . . . . . . 242

E.1 Eigenvalues of Reduced Models using Rayleigh Damping (a) . . . . . . . . . . 244

E.2 Eigenvalues of Reduced Models using Rayleigh Damping (b) . . . . . . . . . . 245

E.3 Eigenvalues of Reduced Models using Rayleigh Damping (c) . . . . . . . . . . 246

E.4 Eigenvalues of Reduced Models using Rayleigh Damping (d) . . . . . . . . . . 247 


\section{List of Acronyms}

FEM Finite Element Method

RLC (R)esistor-(I)nductor-(C)apacitor Resonant Circuit

SOAR Second Order Arnoldi

VLSI Very Large Scale Integration 


\section{List of Symbols}

\begin{tabular}{|c|c|}
\hline$A$ & First-order system matrix \\
\hline$A_{r}$ & Cross-sectional area (SUGAR) \\
\hline$B$ & First-order system matrix \\
\hline$[B]$ & Matrix relating strains and nodal displacements \\
\hline$c$ & Coupling constant \\
\hline$\partial x$ & Relative Error \\
\hline$[d]_{e}$ & Element damping matrix in SUGAR \\
\hline$D$ & Global damping matrix \\
\hline$\left[D^{(e)}\right]$ & Element damping matrix in FEM \\
\hline$D_{c r}$ & Critical damping \\
\hline$E$ & First-order system matrix \\
\hline$E_{m}$ & Young's modulus \\
\hline$F$ & Force vector for a second-order differential equation \\
\hline$G$ & First-order system matrix \\
\hline$h(t)$ & Impulse response \\
\hline$H(s)$ & Transfer function \\
\hline$H_{i}(x)$ & Hermitian matrix \\
\hline$I$ & Identity matrix \\
\hline$I_{m}$ & Moment of Inertia \\
\hline
\end{tabular}

xvii 


\begin{tabular}{|c|c|}
\hline$k_{n}$ & Zero of a system \\
\hline$[k]_{e}$ & Element stiffness matrix in SUGAR \\
\hline$\left[K^{(e)}\right]$ & Element stiffness matrix in FEM \\
\hline$K$ & Global stiffness Matrix \\
\hline$l^{T}$ & Incidence matrix of points with applied forces \\
\hline$L$ & Length of beam (SUGAR) \\
\hline$[m]_{e}$ & Element stiffness matrix in SUGAR \\
\hline$m_{n}$ & Moment of a system \\
\hline$M$ & Global mass matrix \\
\hline$M_{0}$ & Moment of rotation \\
\hline$\left[M^{(e)}\right]$ & Element mass matrix \\
\hline$n$ & Number of iterations used to create reduced model \\
\hline$N$ & Number of variables in original state-space representation \\
\hline$\left[N_{i}\right]$ & Shape function matrix \\
\hline$p_{n}$ & Pole of a system \\
\hline$q$ & Orthogonal vector \\
\hline$Q$ & Orthogonal matrix \\
\hline$R$ & Force vector for a first-order differential equation \\
\hline$s$ & Frequency point \\
\hline$u(t)$ & Input vector \\
\hline$u(x)$ & Axial displacement \\
\hline$w$ & Width of beam \\
\hline
\end{tabular}

xviii 


$\begin{array}{ll}W & \text { General square matrix } \\ V & \text { General square matrix } \\ X(s) & \text { Input response in the frequency domain } \\ Y(s) & \text { Output response in the frequency domain } \\ V o l & \text { Volume of tetrahedron } \\ z(t) & \text { Input vector } \\ \rho & \text { Density } \\ \mu & \text { Viscosity constant } \\ \zeta & \text { Damping factor } \\ \alpha & \text { Rayleigh constant } \\ \beta & \text { Rayleigh constant } \\ \kappa & \text { Krylov subspace } \\ \mathfrak{R} & \text { Real space } \\ \mathbb{C} & \text { Complex plane } \\ v(x) & \text { Poisson's ratio } \\ \theta(x) & \text { Angle of rotation } \\ & \end{array}$

$\Delta \quad$ Distance or gap from the device to the substrate 


\section{Definition of Terms}

Actuator

Axial

displacement

Backward Euler method

Castigliano's theorem

Congruent

Transformation

Controllable subspace

Couette flow

Differential equation a mechanical device for moving or controlling a mechanism or system.

displacement along the centerline/axis

an implicit method, meaning that we have to solve an equation to find

$y_{n+1}$, where $y_{n+1}=y_{n}+h f\left(y_{n+1}, t_{n+1}\right)$

the partial derivative of the internal (strain) energy expressed in terms of the beam deflections with respect to the displacement under a concentrated force gives that the value of that force

a transformation of the form $A \rightarrow P^{\mathrm{T}} A P$, where $A$ and $P$ are square matrices, $P$ is invertible, and $P^{\mathrm{T}}$ denotes the transpose of $P$

Given a linear system $\dot{x}=A x+B u$, where $x(t) \in \mathfrak{R} \quad$ and $u(t) \in \mathfrak{R}^{m}$, the system is controllable if from any given condition, $x_{0}$, there exists an input that brings the state to any other state within any given (nonzero) interval

the laminar flow of a viscous fluid in the space between two parallel plates, one of which is moving relative to the other a mathematical equation for an unknown function of one or several 
variables that relates the values of the function itself and of its derivatives of various orders

Discretization transforming continuous models and equations into discrete (individual or separate) parts

Eigenvalue eigenvalues are a special set of scalars associated with a linear system of equations (i.e., a matrix equation) that are sometimes also known as characteristic roots. They are found by solving $\operatorname{det}(A-\lambda I)=0$

Eigenvector an eigenvector of a given linear transformation is a vector which is multiplied by a constant called the eigenvalue during that transformation

Forward Euler an explicit method, i.e., $y_{n+1}$ is given explicitly in terms of known quantities such as $y_{n}$ and $f\left(y_{n}, t_{n}\right)$. Explicit methods are very easy to implement, however, the drawback arises from the limitations on the time step size to ensure numerical stability. The stability criterion for the forward Euler method requires the step size $h$ to be less than 0.2 $y_{n+1}=y_{n}+h f\left(y_{n}, t_{n}\right)$

Gram-Schmidt a method for orthogonalizing a set of vectors in an inner product space, process most commonly the Euclidean space $\mathrm{R}^{n}$. The Gram-Schmidt process takes a finite, linearly independent set $S=\left\{v_{1}, \ldots, v_{n}\right\}$ and generates an orthogonal set $S=\left\{u_{1}, \ldots, u_{n}\right\}$ that spans the same subspace as $S$. 
Hessenberg a is one that is "almost" triangular. To be exact, an upper Hessenberg matrix matrix has zero entries below the first subdiagonal, and a lower Hessenberg matrix has zero entries above the first superdiagonal.

Ill-conditioning occurs when the condition number of a matrix is too large. The condition number is the ratio of the largest to the smallest singular value in the singular value decomposition of a matrix.

Indefinite A form which is neither positive definite nor negative definite is called indefinite.

Inertia a property of matter by which it remains at rest or in uniform motion in the same straight line unless acted upon by some external force

Krylov subspace the Krylov subspace generated by an $n$-by- $n$ matrix, $A$, and an $n$-vector, $b$, is the subspace spanned by the vectors $b, A b, \ldots, A^{n-1} b$.

Linear isotropic linear isotropic theory of elasticity - an isotropic material has point theory of elasticity symmetry. The linear elastic behavior, used to describe the material properties of an element, assumes that strain is proportional to the stress on the element. This assumption explains how deformations appear when stress is applied and how they disappear when stress is removed. Lumped Model a way of simplifying the behavior of spatially distributed systems into a topology consisting of discrete entities that approximate the behavior of 
the distributed system under certain assumptions.

The lumped element model of electronic circuits makes the simplifying assumption that each element is finite point in space, and that the wires connecting elements are perfect conductors.

Moment of inertia

Negative definite Observable subspace

Orthogonal basis

Orthonormal basis

Padé approximant the rotational analog of mass. It is the inertia of a rigid rotating body with respect to its rotation.

a matrix is negative definite when all the eigenvalues are negative Given a linear system $\dot{x}=A x+B u, y=C x+D u$ where $x(t) \in \mathfrak{R}^{n}$, $u(t) \in \mathfrak{R}^{m}$, and $y(t) \in \mathfrak{R}^{p}$ the system is observable if an input and output trajectory, it is possible to uniquely determine the initial state a set of elements whose span is dense in the space, in which the elements are mutually orthogonal a set of elements whose span is dense in the space, in which the elements are mutually orthogonal and of magnitude one i.e. a normalized orthogonal basis the "best" approximation of a function by a rational function of given order. A Padé approximant often gives better approximation of the function than truncating its Taylor series and it may still work where the Taylor series does not converge. For these reasons Padé approximants 
are used extensively in computer calculations. It is found by expanding the Taylor series about a point, $\mathrm{x}_{0}$, and setting the resulting series to zero and solving for the co-efficients.

Parameterization the process of defining or deciding the 'parameters' - usually of some model - that are relevant to the question being asked of that model. If for example the model is of wind turbine with a particular interest in the efficiency of power generation, then the parameters of interest will probably include the number, length and pitch of the blades.

Passivity the ability of a component that consumes but does not produce energy or one that is incapable of power gain. The device being modeled should always maintain stability in its mathematical form and, if it is initially passive, it should remain passive

Poisson's ratio a rod-like specimen subjected to uniaxial tension will exhibit some shrinkage in the lateral direction for most materials. The ratio of lateral strain and axial strain is defined as Poisson's ratio.

Positive definite a positive-definite matrix is a matrix, $M$, with all eigenvalues $\lambda_{i}$ positive.

QR decomposition (also called the QR factorization) of a matrix is a decomposition of the matrix into an orthogonal and a triangular matrix. 
Stability (in control theory) often means that for any bounded input over any amount of time, the output will also be bounded.

State space representation a mathematical model of a physical system as a set of input, output and state variables related by first-order differential equations The state space representation (also known as the "time-domain approach") provides a convenient and compact way to model and analyze systems with multiple inputs and outputs.

Stiffness the capacity of a mechanical system to sustain loads without excessive changes of its geometry (deformations).

Steady state a situation in which all state variables are constant in spite of ongoing processes that strive to change them.

Subspace a subset of a vector space that is closed under addition and scalar multiplication

System-on-a- System-on-a-chip or system on chip (SoC or SOC) refers to integrating chip all components of a computer or other electronic system into a single integrated circuit (chip)

Transducer devices which transform an input signal (mainly an electrical signal) into motion eg motor, micromirror

Transient A transient event is a short-lived oscillation in a system caused by a 
sudden change of voltage, current or load

Transverse deflection

Viscosity

Young's modulus the movement resulting that is perpendicular to the direction of the

applied force, eg if the beam is displaced in $\mathrm{x}$, the movement in the $\mathrm{yz}$ plane is the transverse deflection.

a measure of the resistance of a fluid to being deformed by either shear stress or extensional stress. It is commonly perceived as "thickness", or resistance to flow.

The modulus of elasticity in tension, also known as Young's modulus $E$, is the ratio of stress to strain on the loading plane along the loading direction. It is a measure of stiffness. 


\section{Chapter 1}

\section{Introduction}

\subsection{Background and Motivation}

The modeling of complex engineering, medical, and chemical systems is an extremely relevant and interesting area of study as it allows for numerically accurate mathematical approximations of these systems. Many of these systems can be described by second-order differential equations, such as those in structural dynamics, aeronautics, control systems, circuit simulations, biological and biochemical processes, and micro-electromechanical systems(MEMS) to name a few [1].

The size of these mathematical models, more commonly obtained with modeling techniques such as Finite Element Analysis and Finite Differences, is often too large for any further numerical analysis and as such, analysis and design problems become difficult or impossible to implement and solve within easily available resources [2]. There is obviously a clearly defined need to be able to form small accurate representations of these large models to achieve rapid design for easy analysis of the response of these systems in order to reduce the overall cycle time from design and testing to manufacturing and implementation. The most efficient technique for 
this computer aided design is known as model reduction.

As the complexity of engineering devices increases, the ability to model these systems quickly and accurately is increasingly important. However, with increasing complexity comes the need for highly refined discretization techniques resulting in extremely large numerical models. The result is an exponential need for computational power for the simplest of calculations, and even the ability to store the original information representing these models in memory can become impossible. This is the main reason why model reduction of complex systems is a beneficial tool in the scientific community.

Currently, many model reduction techniques implemented are primarily focused on modeling first order differential equations and thus, folding higher order systems into an equivalent mathematical companion form first order system. While this provides a good solution for simpler systems and is extremely useful in circuit simulations, the resulting companion form for second-order systems can often lead to instabilities and loss of the physical meaning of the original more complex systems. Another issue that arises is that many model reduction techniques do not have clearly defined desired error thresholds or number of moments that should be taken according to the size of the model or even when scaling of system matrices is needed, leading to educated guesses at some stage in the process. This, in itself, poses its own engineering pitfalls.

As the growing trend toward highly sophisticated electrical circuits, devices and systems continues, the need for a more concise and accurate model reduction technique becomes more apparent.

The main goal of this thesis is to examine the stability and robustness of model reduction techniques based on Krylov subspace methods. More specifically, the Arnoldi algorithm and 
the Second Order Arnoldi (SOAR) procedure when used to model the mechanical aspects of micro-electromechanical systems (MEMS). In particular, two different modeling techniques, Finite Element Method (FEM) modeling and a tool created by the Berkeley Sensor and Actuator Center at the University of California at Berkeley, SUGAR, will be used as tools to model MEMS. FEM is commonly used in structural engineering and allows for the modeling of highly complex structures. SUGAR was created to allow for the modeling of MEMS systems with many interconnected devices. This thesis will assess criteria, such as stability and accuracy and address the effect of damping which is often overlooked by researchers, and attempt to identify the criteria and conditions better suited to each method of modeling and model reduction technique.

\subsection{Contribution}

Micro-electromechanical systems (MEMS) are often modeled using complex mathematical techniques via partial and ordinary second-order differential equations, which allows designers the ability to couple different physical aspects of the device, such as heat transfer, electrostatics, and fluid dynamics. Consequently, mathematical models representing MEMS are very complex and with high complexity comes high dimension, so modeling techniques, like FEM, produce large models to accurately define the device.

Consequently, the main topic of this thesis is the modeling of MEMS structures, represented by second-order differential equations via two modeling techniques: FEM and SUGAR, and applying model reduction techniques based on Krylov subspace methods, the Arnoldi algorithm and the SOAR procedure. The main contributions will be: 
1. A review of the stability of the model reduction techniques by looking at existing mathematical tests that have been defined in the literature to ensure stability and passivity. A focus will be placed on reforming the second-order differential equation into a first order companion form and the resulting stability and passivity of the new system.

2. Extensive numerical analysis in the time domain and the frequency domain will be conducted to survey the performance of the model reduction techniques. The dimensions of models as well as the damping will be varied in order to show any benefits of either model reduction technique. Also, the MEMS will be modeled with both FEM and SUGAR and all of the above the analysis will be performed using the two types of modeling techniques.

3. The contribution of damping on model reduction is examined with an emphasis on how it affects the stability of model reduction. In addition, a procedure which simplifies the model reduction process, is very robust and eliminates the need to generate more than one orthogonal matrix, will be discussed and numerically examined.

\subsection{Organization of Thesis}

This thesis provides a review of MEMS, modeling techniques and the standard concepts in model reduction, with a focus on Krylov-based model reduction methods for implementing second-order systems: model reduction based on the companion form of the second-order system using the Arnoldi Algorithm and direct model reduction of the second-order system using a Second Order Arnoldi (SOAR) method. 
The stability criteria for model reduction and which methods provide a better basis for guaranteeing stability and passivity of the reduced model will be examined, as well as any existing conditions that must be taken into account for stability. A more thorough investigation of this result will be performed through extensive simulations of a basic cantilever structure, of varying model sizes, in the frequency and time domain.

The role of damping in model reduction will then be examined and the behavior of both SOAR and Arnoldi with increasing complexity will also be considered. Finally, an observation that makes the Arnoldi algorithm more robust will be discussed. 


\section{Chapter 2}

\section{Micro-electromechanical Systems and Modeling Techniques}

\subsection{Micro-electromechanical Systems}

Micro-electromechanical Systems (MEMS) are the integration of mechanical elements, sensors, actuators, and electronics on a common silicon substrate through microfabrication technology [3]. The fabrication of MEMS systems is done in essentially two parts: 1) electronic components are fabricated using integrated circuit processes and 2) the mechanical components are fabricated using micromachining processes that selectively etch away parts of the substrate, usually silicon, or add new structural layers to form the mechanical and electromechanical devices. For example, the micro-mirror shown in Figure 2.1, is a simple MEMS structure where electronics and micromachining are combined. The micro-mirror is electrostatically actuated and is supported on a torsional beam with two separate electrodes connected at each end of the mirror, allowing it to be tilted about the axis of the torsion beam. The mirror acts as its own electrode. This device has applications in display technologies and as optical switches. 


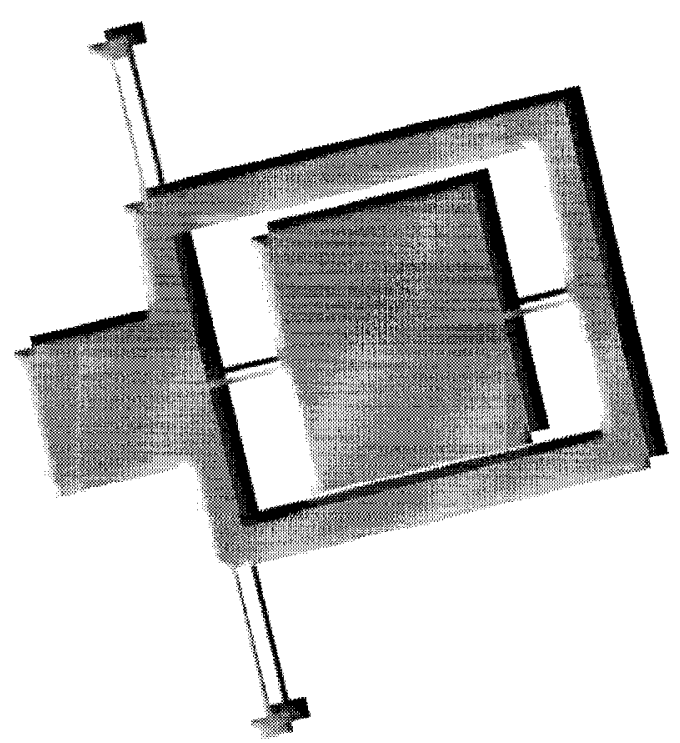

Figure 2.1: Micro-mirror MEMS system

MEMS are found extensively in all aspects of modern technology where systems-on-a-chip are becoming more commonly used for all types of applications including telecommunication circuits, computers, cell phones, and cameras, to name a few. As everyday users become more sophisticated in their need for smaller devices with more integrated features, the extent to which MEMS and NEMS (nano-electromechanical systems) are used is growing exponentially, as is the complexity of these structures [4]. Designers must now take into consideration coupled domain problems that arise from amalgamating so many different features in very small feature sizes, for example the design of a microscale thermal anemometer couples heat transfer, electrostatics, and fluid dynamics [4].

It is evident that good modeling tools to allow designers to test and integrate features before actual fabrication are very important. This is where modeling techniques such as the Finite Element Method (FEM), Finite Difference Methods, Beam Theory, and Finite Volume Methods, 
can be very useful.

At the systems engineering level, the physical response of MEMS can be characterized by mathematical formulations represented by ordinary and partial differential equations in which the most essential properties of the device are included, such as thermal and material properties. These mathematical models can be extended to include the coupling of domains, thus allowing designers to incorporate many features in their design considerations, and still allow MEMS to be effectively modeled through several available techniques including those stated previously.

One of the most useful representation of many MEMS is the general second-order differential equation of motion given by:

$$
\begin{gathered}
M \ddot{z}(t)+D \dot{z}(t)+K z(t)=F u(t) \\
y(t)=l^{T} z(t)
\end{gathered}
$$

where $M, D$, and $K$ are the mass, damping, and stiffness matrices respectively, $F$ is the excitation vector, and $y(t)$ is the output vector, $u(t)$ is the input vector and $l^{T}$ is the (generalized) incidence matrix of points with applied forces, thermal input, currents and so on.

Devices modeled in this thesis will focus on MEMS represented by the general discretized second-order differential equation stated above, as the primary goal here is to evaluate model reduction techniques based on Krylov subspaces with regards to the mechanical properties of MEMS.

This thesis will initially look at a simple MEMS cantilever structure modeled primarily in FEM and then using a new modeling tool called SUGAR. Other real-world MEMS structures will then be modeled in FEM, including a serpentine device which is sensitive to very small forces due to its structure, thus making it useful for low frequency applications; an actuator which is a device for moving or controlling the motion of a mechanism or system; and 
a resonator which is a device that exhibits resonance at particular frequencies and is used in telecommunication devices and acoustic systems.

These structures are shown in Figure 2.2, where the fixed ends are indicated by the red spheres and yellow spheres indicate where the forces are applied to the structure.

The purpose of modeling these more complex structures is to examine how well modeling and model reduction techniques match the real system.

\subsection{Finite Element Modeling}

Finite element method (FEM) modeling is a method initially developed for the numerical solution of complex structures in structural mechanics, but has been extended for use in many modern applications, including the accurate analysis of the static and dynamic behavior of complex structures at the micron and nanometer scale. Finite element analysis (FEA) achieves accurate simulation of the desired system by defining the structure through a set of finite elements that are interconnected by nodes, which in turn define a grid or mesh.

Elements may have varying physical properties such as thickness, density, Young's modulus, moment of inertia, Poisson's ratio, and thermal properties to name a few. Structures can also be modeled with different types of elements, depending on the nature of the problem and its complexity. These elements can be simple one dimensional elements with one node at each end, suitable for modeling trusses, beams, and cables, or highly complex 3-D elements such as tetrahedrons and hexahedrons, which are more suitable for modeling engineering structures such as aircraft components and MEMS. 
The resulting mesh created by the interconnected nodes contains these material and structural properties of the system, allowing the response of the structure to different environmental and loading conditions to be accurately achieved. The importance of this lies in the design and validation process of these complex engineering systems, leading to savings in both time and cost.

The main meshing technique used in this thesis is finite element methods (FEM). In fact, an in-house developed FEM tool was extended to generate all the models for which model reduction was performed. This program was integrated with a mechanical simulation tool, also built in-house at Carleton University, Department of Electronics, called ATAR. ATAR is used for the decomposition of the models initially into linear blocks which were then decomposed into three dimensional linear FEM tetrahedron elements with four nodes and three degrees of freedom, in $x, y$ and $z$, such as that shown in Figure 2.4. As noted, the nodes of the mesh interconnect elements, and the three degrees of freedom allows the elements to move in three dimensions. Each block contains twelve tetrahedrons, see Figure 2.3.

Once the mesh is formed, a FEM model is then defined by applying boundary conditions to the mesh as a mesh only defines the topology, connectivity and geometry of a structure. The MEMs models generated with FEM are specified with boundary conditions that fix one part of the structure and applies a force of $5 \mu N$ at the free ends. This is more clearly seen in Figure 2.2.

Although the choice of the linear tetrahedron presents many disadvantages over higher order isoparametric elements in that curved boundaries become increasingly complicated to model, which leads to a simple closed form evaluation of the element stiffness matrix and force vector being impossible, the primary focus of this thesis will be on model reduction techniques using 
Krylov subspace methods. The choice of the linear tetrahedron for modeling the MEMs structures presented here was mainly due in part to the ability to test and use the existing modeling tool already available, so the mechanical modeling will focus on the dynamic analysis of these MEMs structures to determine displacements and forces.

\subsubsection{Basic FEM theory}

A comprehensive review of FEM is beyond the scope of this thesis, so a summary of the key aspects of an element formulation and the general formation of the resulting FEM mesh will be discussed [5].

MEMS structures are generally described by the parametrized ordinary differential equation

$$
\sum_{N}:\left\{\begin{array}{c}
M \ddot{z}(t)+D \dot{z}(t)+K z(t)=F u(t) \\
y(t)=l^{T} z(t)
\end{array}\right.
$$

where $M, D$, and $K$ are the mass, damping, and stiffness matrices used in static and dynamic analysis and $F$ defines the loading or applied forces on the structure, $y(t)$ is the output vector, $u(t)$ is the input vector and $l^{T}$ is the incidence matrix of points with applied forces, thermal input, currents and so on. As stated previously, the FEM tool used to model the MEMS structures here implements the linear tetrahedron element for decomposing MEMS structures into a mesh or grid. Figure 2.3 illustrates the tetrahedron containing the four nodes or coordinates that define a single element. These coordinates specify the location of the element in the entire mesh or global matrix, and carries important information about the size of the mesh defined by the element. The basic steps in FEM are first to find the element mass, $\left[M^{(e)}\right]$, stiffness, $\left[K^{(e)}\right]$, and damping, $\left[D^{(e)}\right]$, matrices for each element in the matrix, then assemble the elements into a global matrix that characterizes the MEMS structure being considered [5]. 
An element mass matrix, $\left[M^{(e)}\right]$, for a tetrahedron is defined as

$$
\left[M^{(e)}\right]=\iint_{V o l^{(c)}} \int \rho[N]^{T}[N] d V o l
$$

where $\rho$ is the density of the material, $V o l$ is the volume of the structure, and $N$ is the number of elements the model has been discretized into.

Equation 2.2 .2 can be simplified to the $12 \times 12$ matrix

$$
\left[M^{(e)}\right]=\frac{\rho V o l^{(e)}}{20}\left[\begin{array}{cccccccccccc}
2 & 0 & 0 & 1 & 0 & 0 & 1 & 0 & 0 & 1 & 0 & 0 \\
0 & 2 & 0 & 0 & 1 & 0 & 0 & 1 & 0 & 0 & 1 & 0 \\
0 & 0 & 2 & 0 & 0 & 1 & 0 & 0 & 1 & 0 & 0 & 1 \\
1 & 0 & 0 & 2 & 0 & 0 & 1 & 0 & 0 & 1 & 0 & 0 \\
0 & 1 & 0 & 0 & 2 & 0 & 0 & 1 & 0 & 0 & 1 & 0 \\
0 & 0 & 1 & 0 & 0 & 2 & 0 & 0 & 1 & 0 & 0 & 1 \\
1 & 0 & 0 & 1 & 0 & 0 & 2 & 0 & 0 & 1 & 0 & 0 \\
0 & 1 & 0 & 0 & 1 & 0 & 0 & 2 & 0 & 0 & 1 & 0 \\
0 & 0 & 1 & 0 & 0 & 1 & 0 & 0 & 2 & 0 & 0 & 1 \\
1 & 0 & 0 & 1 & 0 & 0 & 1 & 0 & 0 & 2 & 0 & 0 \\
0 & 1 & 0 & 0 & 1 & 0 & 0 & 1 & 0 & 0 & 2 & 0 \\
0 & 0 & 1 & 0 & 0 & 1 & 0 & 0 & 1 & 0 & 0 & 2
\end{array}\right]
$$

Similarly, the element damping matrix, $\left[D^{(e)}\right]$, is defined as

$$
\left[D^{(c)}\right]=\iint_{V o l} \int \mu[N]^{T}[N] d V o l
$$

and is the same as the element mass matrix but with a scaling factor of the viscosity, $\mu$, instead of the density, $\rho,[5]$.

The element stiffness matrix, $\left[K^{(c)}\right]$, is more complicated and must be carefully constructed for each element. The direction in which the nodes are defined for each element must be the same for all calculations involved. 
It is defined as

$$
\left[K^{(e)}\right]=\iint_{V o l} \int[B]^{T}[C][B] d V o l
$$

where

$$
[B]=\frac{1}{6 V o l}\left[\begin{array}{cccccccccccc}
b_{i} & 0 & 0 & b_{j} & 0 & 0 & b_{k} & 0 & 0 & b_{l} & 0 & 0 \\
0 & c_{i} & 0 & 0 & c_{j} & 0 & 0 & c_{k} & 0 & 0 & c_{l} & 0 \\
0 & 0 & d_{i} & 0 & 0 & d_{j} & 0 & 0 & d_{k} & 0 & 0 & d_{l} \\
c_{i} & b_{i} & 0 & c_{j} & b_{j} & 0 & c_{k} & b_{k} & 0 & c_{l} & b_{l} & 0 \\
0 & d_{i} & c_{i} & 0 & d_{j} & c_{j} & 0 & d_{k} & c_{k} & 0 & d_{l} & c_{l} \\
d_{i} & 0 & b_{i} & d_{j} & 0 & b_{j} & d_{k} & 0 & b_{k} & d_{l} & 0 & b_{l}
\end{array}\right]
$$

where

$$
a_{i}=\left[\begin{array}{ccc}
x_{j} & y_{j} & z_{j} \\
x_{k} & y_{k} & z_{k} \\
x_{l} & y_{l} & z_{l}
\end{array}\right], b_{i}=-\left[\begin{array}{ccc}
1 & y_{j} & z_{j} \\
1 & y_{k} & z_{k} \\
1 & y_{l} & z_{l}
\end{array}\right], c_{i}=-\left[\begin{array}{ccc}
x_{j} & 1 & z_{j} \\
x_{k} & 1 & z_{k} \\
x_{l} & 1 & z_{l}
\end{array}\right], d_{i}=-\left[\begin{array}{ccc}
x_{j} & y_{j} & 1 \\
x_{k} & y_{k} & 1 \\
x_{l} & y_{l} & 1
\end{array}\right]
$$

The other constants are defined by the cyclic interchange of the subscripts in the order $l, i, j$, and $k[5]$.

$$
[C]=\frac{E_{m}}{(1+v)(1-2 v)}\left[\begin{array}{cccccc}
(1-v) & v & v & 0 & 0 & 0 \\
0 & (1-v) & v & 0 & 0 & 0 \\
0 & 0 & (1-v) & 0 & 0 & 0 \\
0 & 0 & 0 & \frac{(1-2 v)}{2} & 0 & 0 \\
0 & 0 & 0 & 0 & \frac{(1-2 v)}{2} & 0 \\
0 & 0 & 0 & 0 & 0 & \frac{(1-2 v)}{2}
\end{array}\right]
$$

where $E$ represents the Young's modulus, and $v$ Poisson's ratio.

Since $[B]$ and $[C]$ are independent of $x, y$, and $z$ the stiffness matrix can be obtained as follows

$$
\left[K^{(e)}\right]=V^{(e)}[B]^{T}[D][B] d V o l
$$


with $V o l^{(e)}$ representing the volume element

$$
V o l^{(e)}=\frac{1}{6}\left[\begin{array}{cccc}
1 & x_{i} & y_{i} & z_{i} \\
1 & x_{j} & y_{j} & z_{j} \\
1 & x_{k} & y_{k} & z_{k} \\
1 & x_{l} & y_{l} & z_{l}
\end{array}\right]
$$

Once the element matrices are calculated, the FEA method then assembles each element matrix into the global mass, damping and stiffness matrices. A detailed methodology of this process can be found in [5].

Figure 2.5 shows a cantilever after the assembly process.

The global matrices formed from modeling MEMS with FEM are very large, sparse, and symmetrical as can be seen in Figure 2.6. However, despite the sparsity of the matrices, the large dimensions make fast and efficient numerical computations difficult to perform. In addition, some mathematical algorithms such as a Gaussian elimination, can introduce non-zero elements into the system where there were previously none, resulting in matrices that grow increasing dense with any further numerical manipulations. The consequence of denser matrices is greater computational times and the need for greater computer memory allocation.

This is where model reduction techniques play a key role in the successful analysis of MEMS realized through mathematical modeling techniques such as FEM. Model reduction algorithms utilize the symmetry and sparsity of the global matrices and captures the most crucial information about the system via a projection to a much smaller model of the original system. 


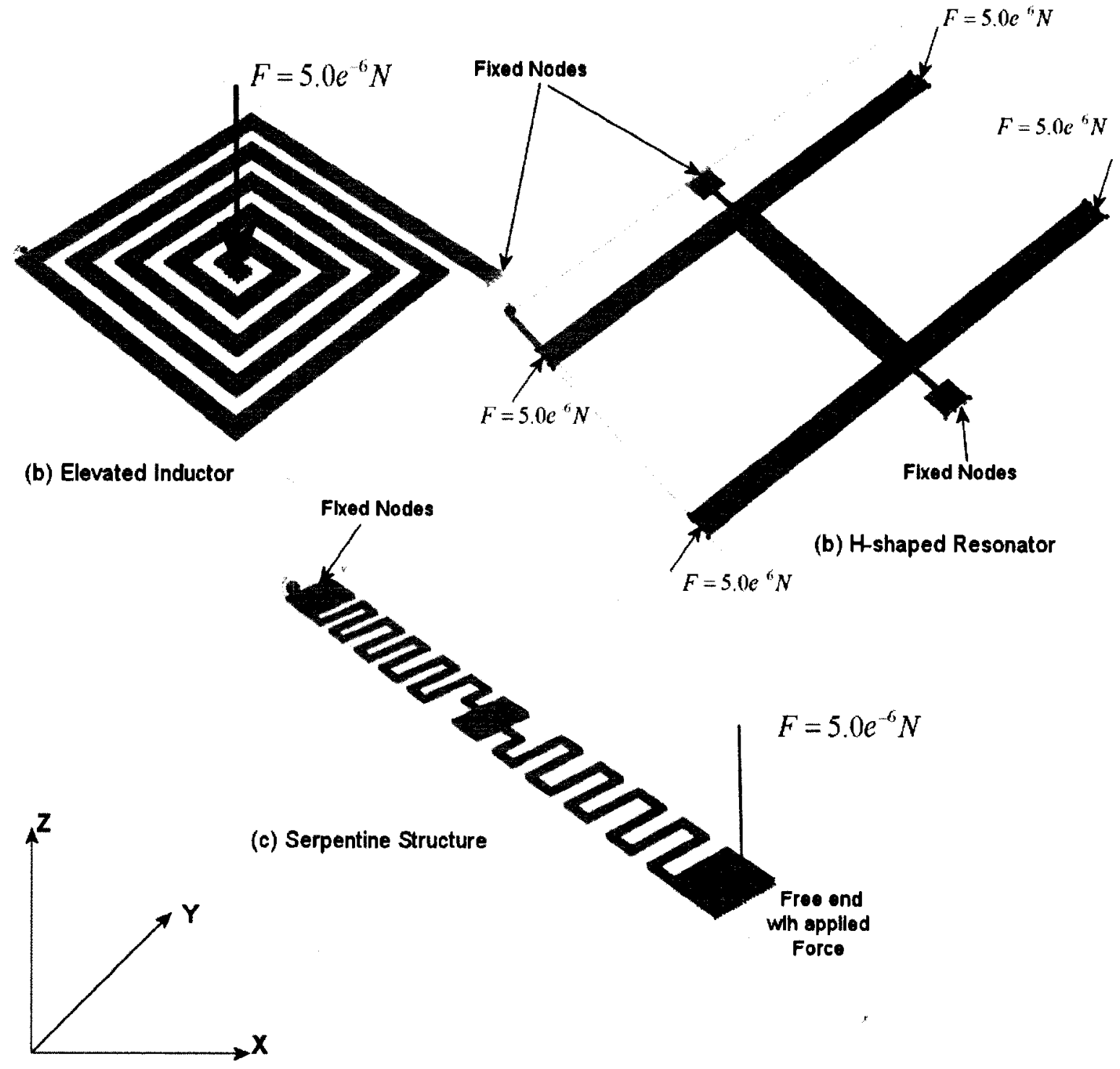

Figure 2.2: Modeled MEMS Structures 


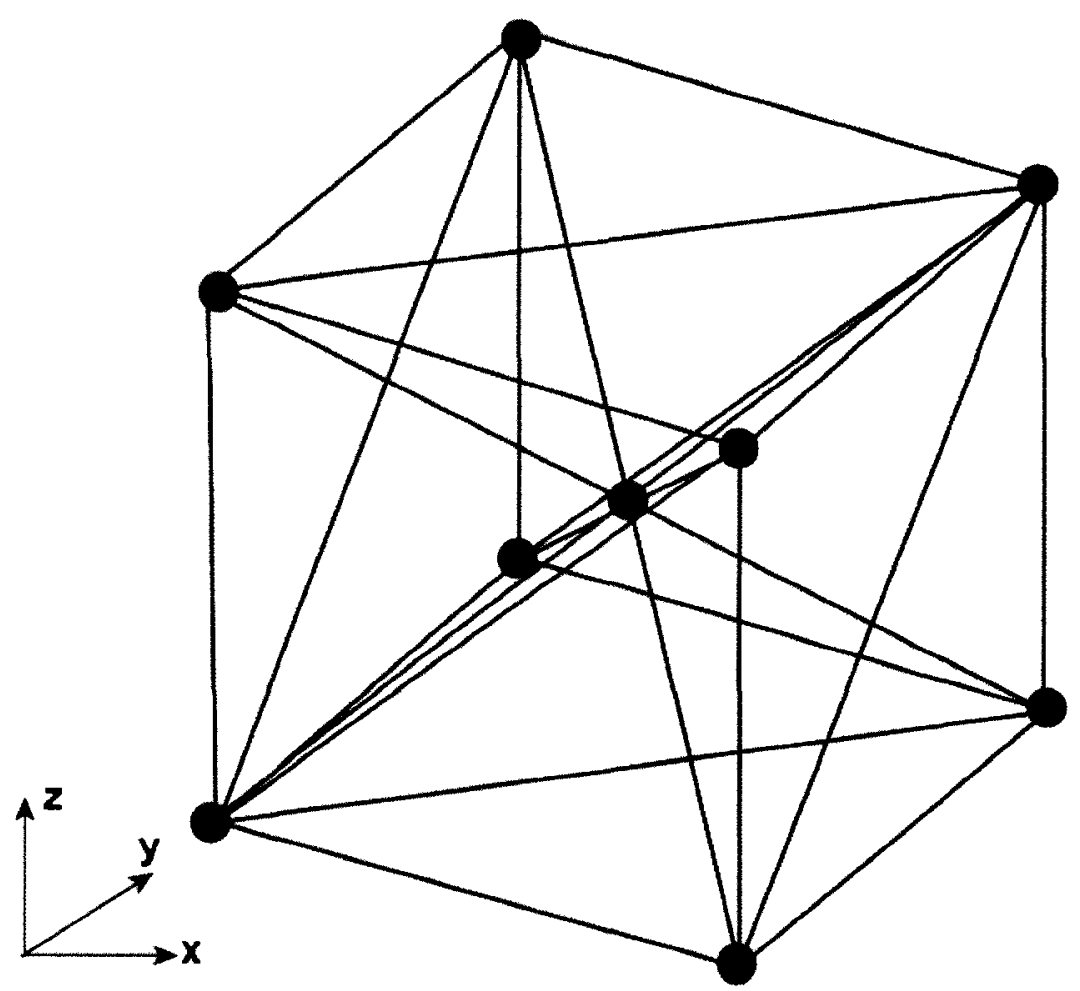

Figure 2.3: One Block decomposed into Tetrahedron Elements

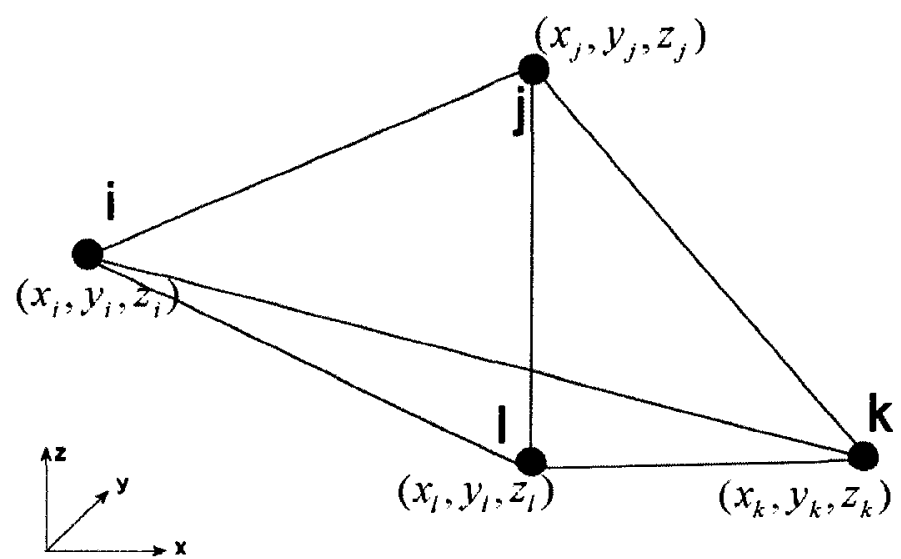

Figure 2.4: The Linear Tetrahedron Element 


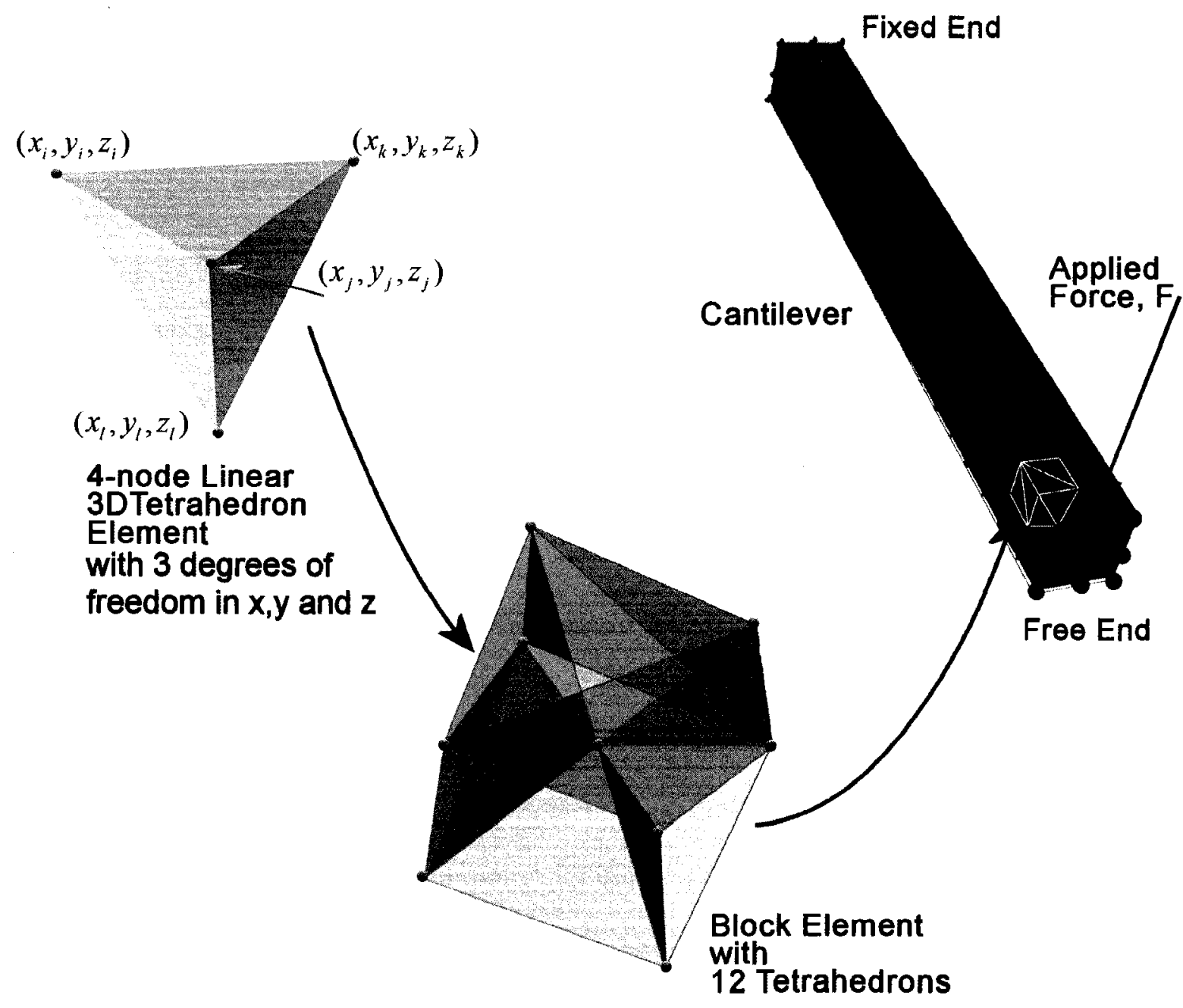

Fixed Nodes

Nodes with applied force

Figure 2.5: Linear Tetrahedron Elements Assembled into a Cantilever 


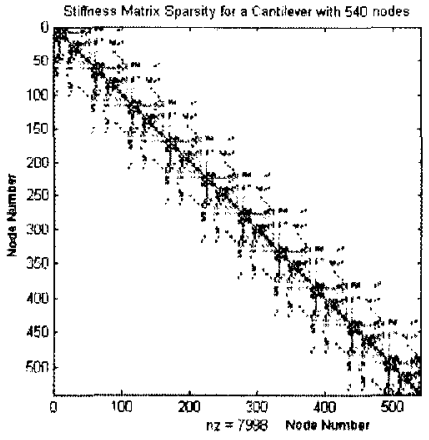

(a) Sparsity of $\mathrm{K}$

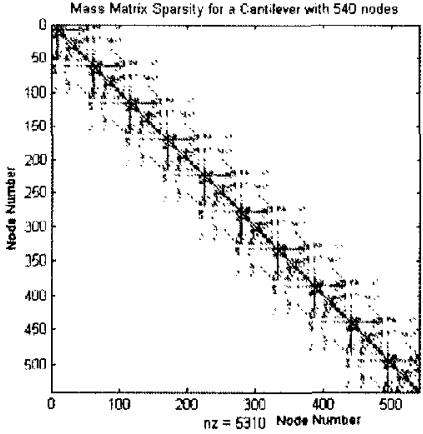

(b) Sparsity of M

Figure 2.6: Sparsity of MEMS Representative matrices modeled using FEM 


\subsection{SUGAR Modeling}

SUGAR is a modeling tool developed at the Berkeley Sensor and Actuator Center at the University of California at Berkeley [6], [7], [8], [9] to perform static, steady state, modal, and transient analysis of three dimensional structures and electrical circuits. SUGAR is based on Euler-Bernoulli beam theory which is a simplification of the linear isotropic theory of elasticity and provides a means of calculating the load-carrying and deflection characteristics of beams [10].

The driving force behind the development of SUGAR was to create a set of algorithms that could provide a modeling tool for higher system level simulations rather than device level simulation. SUGAR is targeted to designers who implement systems that integrate a very large number of components. Finite element analysis is excellent for modeling single components, but the dimensional issues posed by FEM models means that the analysis of a system described by thousands of components is currently very difficult to perform. SUGAR strives to overcome these issues and is an attractive alternative.

The approach SUGAR takes to solving this challenge can be likened to a circuit approach to MEMS design with the emphasis being on hierarchical design, system simulation, and layout synthesis. Its aim is to quickly and accurately simulate large numbers of interconnected MEMS devices [6].

As noted above, many MEMS can be represented by lumped models and their performance can be described by parameterized ordinary differential equations as in equation 2.2.1, which contains the structural properties of the device such as material properties and device geometry. 
Interconnected MEMS devices, built with a tool such as SUGAR, are linked together much in the same way as finite element models are, with nodes. However, unlike FEM, SUGAR models each device by breaking down its structure into simple beam elements connected at each end with a single node.

\subsubsection{Overview of SUGAR Modeling Theory and Implementation}

The basic beam element described in the implementation of SUGAR is shown below in Figure 2.7. In SUGAR [6], when two beams are coupled together their common interconnecting

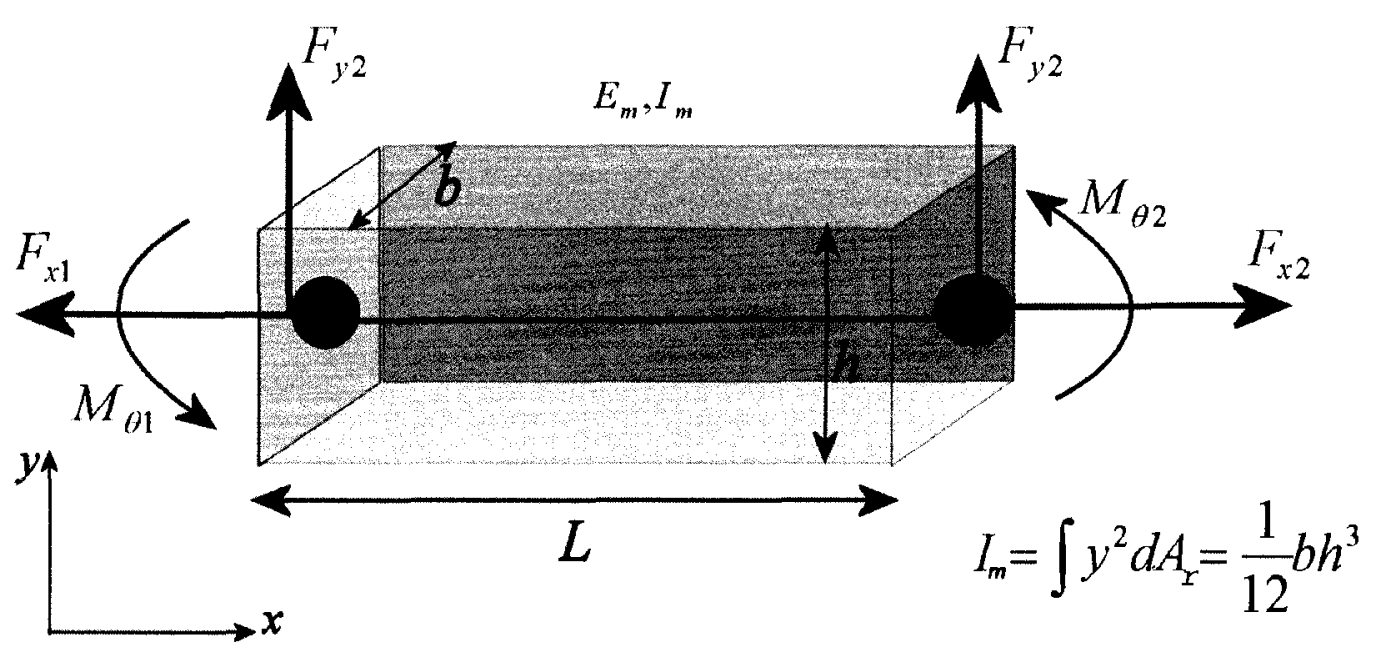

Figure 2.7: Beam Element used in SUGAR

node have the same deflection and slope, thus satisfying continuity conditions. The beam's transverse deflection, $v(x)$, axial displacement, $u(x)$ and angle of rotation, $\theta(x)$ are described by three degrees of freedom at each node as shown in Figure 2.7, and the equation of equilibrium for the area between interconnecting nodes is described by equation 2.3.1.

$$
\frac{\partial^{4}}{\partial x^{4}} v(x)=0
$$


Solving for $v(x)$ and imposing boundary conditions at the end nodes to solve for the coefficients of $v(x)$ and $\theta(x)$ results in

$$
v(x)=H_{1}(x) v_{1}+H_{2}(x) \theta_{1}+H_{3}(x) v_{2}+H_{3}(x) \theta_{2}
$$

where the cubic functions $H_{i}(x)$ are the Hermitian shape functions.

The stiffness of the beam can be obtained using the Castigliano's theorem

$$
F_{i}=\frac{\partial S}{\partial p_{i}}
$$

where $F_{i}$ is the force or moment, $p_{i}$ is the displacement coordinate for degree of freedom $i=$ $1,2,3$ for $p=x, y, \theta$ respectively.

The strain energy, $S$, for a beam with uniform cross-section and considering a linear system is given as

$$
S=\frac{E_{m} I_{m}}{2} \int_{0}^{L}\left(\int_{0}^{L} \frac{\partial^{2}}{\partial x^{2}} v(x)\right)^{2} d x+\frac{E_{m} A_{r}}{2} \int_{0}^{L}\left(\int_{0}^{L} \frac{\partial^{2}}{\partial x^{2}} u(x)\right)^{2}
$$

To find the stiffness in terms of the strain energy equation 2.3.2 is substituted into equation 2.3.4, and equation 2.3.3 is then applied, resulting in equation 2.3.5

$$
k_{i j}=E_{m} I_{m} \int_{0}^{L} H_{i}^{\prime \prime}(x) H_{j}^{\prime \prime}(x) d x+E_{m} A_{r} \int_{0}^{L} H_{i}^{\prime}(x) H_{j}^{\prime}(x) d x
$$

which simplifies to yield the element stiffness matrix as follows,

$$
[k]_{e}=\frac{E_{m}}{L^{3}}\left[\begin{array}{cccccc}
A_{r} L^{2} & 0 & 0 & -A_{r} L^{2} & 0 & 0 \\
0 & 12 I_{m} & 12 E_{m} I_{m} & 0 & -12 I_{m} & -6 I_{m} L \\
0 & 6 I_{m} L & 6 E_{m} I_{m} L & 0 & 6 I_{m} L & 2 I_{m} L \\
-E_{m} A_{r} L^{2} & 0 & 0 & A_{r} L^{2} & 0 & 0 \\
0 & -12 I_{m} & -12 E_{m} I_{m} & 0 & 12 I_{m} & -6 I_{m} L \\
0 & 6 I_{m} L & 6 E_{m} I_{m} L & 0 & -6 I_{m} L & 4 I_{m} L
\end{array}\right]
$$


where $E_{m}$ is Young's modulus, $A_{r}$ is the cross-sectional area of the beam, $I_{m}$ is the moment of inertia, and $L$ is the length of the beam.

A mass matrix is found by equating internal and external work due to virtual displacements [6], giving

$$
m_{i j}=E_{m} I_{m} \int_{0}^{L} \rho(x) A_{r}(x) H_{i}(x) H_{j}(x) d x
$$

where $\rho x$ is the density of the beam. This simplifies into the element mass matrix for a uniform beam with $\rho A_{r}$ being constant, as shown in equation 2.3 .8

$$
[m]_{e}=\frac{\rho A_{r} L}{420}\left[\begin{array}{cccccc}
140 & 0 & 0 & 70 & 0 & 0 \\
0 & 156 & 22 L & 0 & 54 & -13 L \\
0 & 22 L & 4 L^{2} & 0 & 13 L & -3 L^{2} \\
70 & 0 & 0 & 140 & 0 & 0 \\
0 & 54 & 13 L & 0 & 156 & -22 L \\
0 & -13 L & -3 L^{2} & 0 & -22 L & 4 L^{2}
\end{array}\right]
$$

Similarly, the element damping matrix is found by using a simple Couette flow model

$$
[d]_{e}=\frac{\mu L w}{420 \Delta}\left[\begin{array}{cccccc}
140 & 0 & 0 & 70 & 0 & 0 \\
0 & 156 & 22 L & 0 & 54 & -13 L \\
0 & 22 L & 4 L^{2} & 0 & 13 L & -3 L^{2} \\
70 & 0 & 0 & 140 & 0 & 0 \\
0 & 54 & 13 L & 0 & 156 & -22 L \\
0 & -13 L & -3 L^{2} & 0 & -22 L & 4 L^{2}
\end{array}\right]
$$

where $\mu$ is the viscosity of the fluid environment, and $\Delta$ is the distance or gap from the device to the substrate. The damping matrix for SUGAR is similar to that formed in modeling with FEM in that they are both scaled mass matrices. However, the scaling factors for SUGAR and FEM are different. Consequently, to demonstrate underdamping, critical damping and overdamping for SUGAR models, the magnitude of the scaling factor will be different for SUGAR. 
As with the FEM, each element matrix is assembled into a global matrix which in turn defines the overall structure of the system. This is done by mapping the local coordinate and numbering system of each element matrix to the global system. Figure 2.8 shows 6 SUGAR elements assembled to form a cantilever. Generally, in SUGAR a cantilever can be represented with only one element fixed at one end, but many elements are used here for the purposed of applying model reduction techniques. The overall sparsity of the matrices are shown in Figure 2.9 and the global system matrices form the same representation of the MEMS system modeled in FEM, i.e

$$
\left\{\begin{array}{c}
M \ddot{z}(t)+D \dot{z}(t)+K z(t)=F u(t) \\
y(t)=l^{T} z(t)
\end{array}\right.
$$

where $M, D$, and $K$ are the mass, damping, and stiffness matrices respectively. $F$ is the excitation vector, $y(t)$ is the output vector.

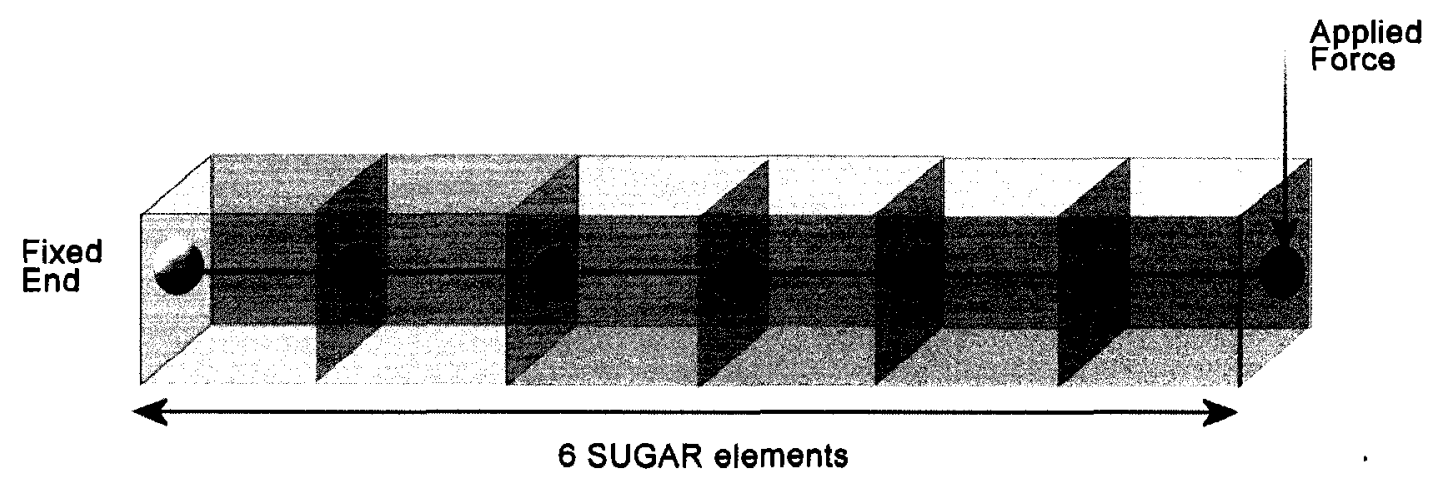

Figure 2.8: Cantilever created with 6 SUGAR Elements

Again, the usefulness of employing model reduction techniques for large SUGAR modeled systems becomes evident, as these techniques can take advantage of the sparsity of such systems as discussed previously. 


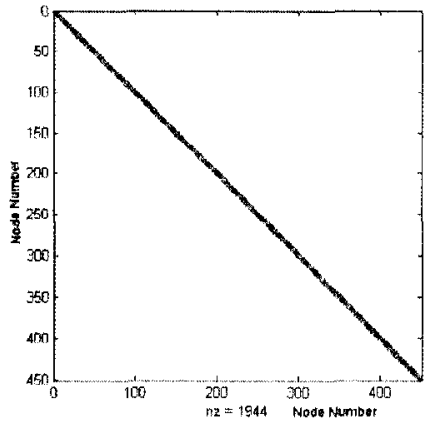

(a) Sparsity of $\mathrm{K}$

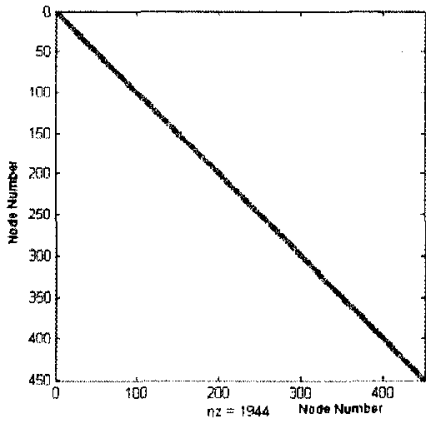

(b) Sparsity of $\mathrm{M}$

Figure 2.9: Sparsity of MEMS Representative matrices modeled using SUGAR 


\subsection{Analysis of MEMS}

From previous discussions, it is evident that modeling MEMS presents a myriad of mathematical and physical considerations that must be taken into account. The coupling of growing complexity, the need for greater accuracy in modeling, and the exponential number of devices and components interconnected in one system, make accurate and comprehensive modeling techniques essential for all these requirements to be met.

This thesis will evaluate the accuracy of model reduction techniques based on Krylov subspace methods by extensively analyzing the response of the reduced model in the frequency and time domain while varying the number of moments and the mechanical damping of the system in modeling mechanical MEMS.

\subsubsection{Frequency Domain Analysis}

Dynamic systems, such as MEMS, are often analyzed in the frequency domain. The main advantage of performing a frequency domain analysis over a time domain analysis is that it is much simpler and does not require additional transient analysis to be performed. Time domain simulators are usually highly complex tools that have the ability to handle difficult mathematical situations by varying the type of algorithm used to achieve a stable response. Simple methods, such as backward Euler, often cannot achieve stable time domain analysis unless the system is very simple or very small time steps are taken. An analysis in the frequency domain provides useful information about many of the structures' important properties, including the range of frequency they operate at within predefined error limits. It also captures important characteristics of a device such as resonant modes. Hence, researchers often choose to perform a frequency 
domain analysis in much of their work on modeling techniques and in model reduction.

However, while a response in the frequency domain can appear to provide numerically stable and accurate results, the frequency domain analysis often fails to capture unstable responses that might actually be relevant to the device when used to examine the response of models that have had model reduction techniques applied. The time domain analysis is a better method for identifying unstable reduced models as will be demonstrated in this thesis.

\subsubsection{Time Domain Analysis}

The time domain response provides a useful tool to conclusively demonstrate the stability of systems and how they respond to varying excitations in time. The analysis done in the time domain was carried out using an in-house software tool called OptiSpice. Initial work was done in Matlab but Optispice was much faster and better suited for the purposes of this thesis.

When modeling any device or component, it is important that the system maintains its original stability and passivity. Passivity refers to the ability of a component that consumes but does not produce energy or one that is incapable of power gain. The device being modeled should always maintain stability in its mathematical form and, if it is initially passive, it should remain passive. The time domain analysis is very important as it is more sensitive to any instability in the system.

In this thesis, particular attention is paid to the time domain analysis of MEMS structures, with special emphasis placed on modeling structures with a range of damping factors, as damping is often left out of many modeling situations. 


\subsubsection{Damping in MEMS}

Damping is the ability of a mechanical system, either deliberately engendered via interactions with outside systems, or inherent to the system, to restrain or diminish any vibrational motion by reducing the amplitude of any oscillations.

In any dynamic system, even at the MEMS level, there is some form of mechanical-energy dissipation producing damping. Generally, in the analysis of these systems, damping is ignored so that dynamic characteristics such as mode shapes or undamped natural frequencies can be studied. However, while damping does play a very important role in the dynamic analysis of structures, because it is so often overlooked, there is a lack of information about the different types of damping present in systems, so exact mathematical representations of these effects do not currently exist [11].

The most effective way to currently treat damping within a modal analysis framework is to use what is commonly known in mathematical modeling as proportional damping, and is formally known as Rayleigh Damping [12], which has the form

$$
D=\alpha M+\beta K
$$

where $D, M$, and $K$ are the damping, mass, and stiffness matrix of the dynamic system. $\alpha$ and $\beta$ are predefined constants.

The main advantage of using Rayleigh damping as the damping matrix is the fact that by using an orthogonal transformation, a structure having $N$-degrees of freedom can be reduced to $n$-number of uncoupled equations [12]. This is directly applicable to the Krylov subspace 
model reduction techniques described in Chapter 3, and the orthogonal $Q$ matrix generated by these techniques.

\section{System Behavior}

For the second-order system described in this thesis, the damping is represented by the first derivative co-efficient, the matrix $D$.

If the system is underdamped, the system oscillates at the natural frequency which is a factor of the damping ratio, $\xi$, defined as

$$
\xi=\frac{D_{i j}}{D_{c r}}=\frac{D_{i j}}{2 \sqrt{M_{i j} K_{i j}}}
$$

where $D_{c r}$ is the critical damping of the system.

An underdamped system has $0<\xi<1$, and is representative of systems that slowly reduces in the magnitude of its oscillations until it reaches an equilibrium or steady state position.

At critical damping $\xi=1$. Critical damping occurs when the system reaches equilibrium as quickly as possible with no oscillations.

When a system is over-damped $\xi>1$, and the system goes slowly to equilibrium but with no oscillations.

Figure 2.10 illustrates an underdamped and a critically damped theoretical cantilever system.

In the time and frequency domain analysis of reduced models of mechanical MEMS, the role and effect of damping will be looked at extensively to determine the relevance of including it in mathematically modeling real structures. The damping used will be modeled using the 


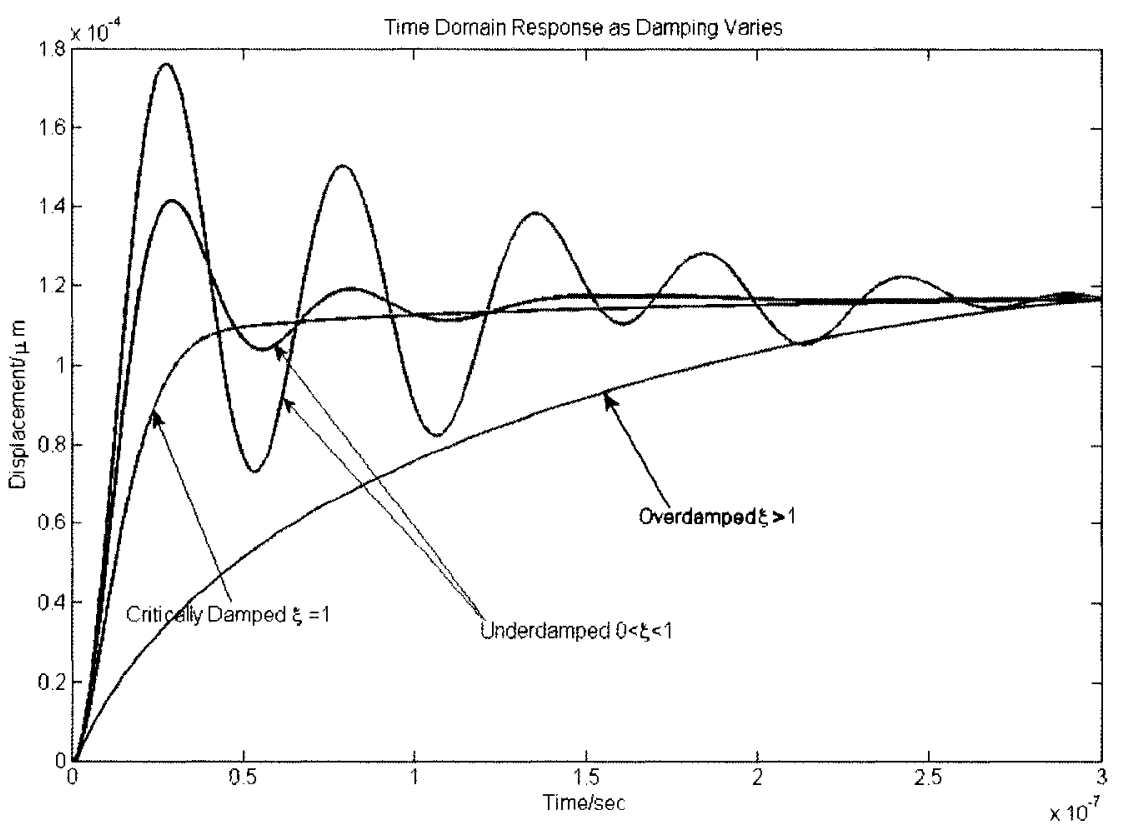

Figure 2.10: Damping Response of an Analytical Cantilever

proportional Rayleigh damping outlined above. 


\section{Chapter 3}

\section{Review of Model Reduction Techniques}

The primary goal of any model reduction technique is to acquire the most accurate representation of the behavior of the original system, and despite the existence of numerous methods for the implementation of model reduction, the main reduction methods fall into three main categories : 1) direct moment matching based on Padé approximation, 2) indirect moment matching based on finding the dominant eigenvalues of a system, and 3) balancing and truncation methods.

Asymptotic Waveform Evaluation (AWE) is an example of explicit or direct moment matching that uses the Padé approximation to extract the dominant poles and residues of the system [13]. However, the Padé approximation technique is known to suffer instability and sensitivity problems [14] due to ill-conditioning problems that can lead to the reduced model lacking passivity.

Indirect or implicit moment matching techniques seek to overcome these issues by extracting the leading eigenvalues instead of the dominant poles of the original system. These methods of model reduction are based on Krylov subspace methods. 
Balancing and truncation methods try to delete (or truncate) the states that contribute smaller amounts of energy to the outputs but need a lot of input energy to change, so that the resulting state-space coordinate transformation of input-to-state map and the state-to-output map are aligned. These methods result in highly accurate approximations to the original systems but the computational demand of these algorithms, coupled with increasing complexity of the systems to be modeled, makes it an impractical solution for the implementation of model reduction [15], [16].

Krylov subspace methods can efficiently compute a basis for the principal controllable and observable subspace [17]. These include Lanczos, Indirect Moment Matching, and SOAR model reduction techniques where the reduced model can be obtained by projecting the statespace on these subspaces. Krylov methods only require matrix-vector multiplications, which makes them extremely attractive and efficient for large-scale systems [16].

For the purposes of this thesis, the focus will be on Krylov subspace methods for model reduction and more specifically indirect moment matching model reduction using the Arnoldi algorithm and the second-order Arnoldi reduction(SOAR) technique.

\subsection{Model Reduction of Second Order Systems}

With larger-scaled systems and increasing complexity, robust and numerically accurate model reduction techniques are becoming very attractive and practical solutions for approximating higher order systems such as those represented by second-order differential equations. Model reduction of these systems must maintain the behavior of the original system in both the time and the frequency domain, and also preserve the physical or mechanical structure of the system 
[18].

Many systems are typically modeled by sets of ordinary differential equations of secondorder or higher, and most model reduction techniques are designed for solving these systems by collapsing them into a state-space model. This transformation results in a set of first-order differential equations to which existing reduction techniques are applied [19].

The reformulation of second-order ordinary differential equations into the first-order model, although mathematically equivalent, presents a number of disadvantages. Mainly, it ignores the physical meaning of the original system matrices because the reduced-order system is no longer second-order, and important properties inherent to the original structure, such as symmetry, are lost. Also, the numerical stability and passivity of the reduced system cannot be guaranteed even if the original system is stable and passive. This occurs because the resulting first-order matrices can be very ill-conditioned, leading to numerical instability and a consequent loss of passivity. Although this can be overcome by scaling, the scaling of matrices for the purposes of model reduction is more of a trial and error process rather than an exact science [1], [20].

In an attempt to overcome the loss of stability and passivity,the use of Krylov subspace methods were proposed to reduce second-order systems while preserving their structure via indirect moment matching techniques and include the Arnoldi algorithm and the Second Order Arnoldi (SOAR) procedure. The Arnoldi algorithm applies a projection onto the Krylov subspace after rewriting the second-order system into a first-order equivalent system, while SOAR applies a projection directly to the second-order model [18]. 


\subsection{Moments of a System}

As the model reduction techniques being examined are based on indirect moment matching techniques, the moments of a system will be defined here.

Consider the Taylor series of the transfer function $H(s)$ at a point $s=0$. In the $s$ domain, the transfer function of a linear network, $H(s)$, is defined as the ratio of the output to the input under zero initial conditions:

$$
H(s)=\frac{Y(s)}{X(s)}
$$

If $H(s)$ is expanded around $s=0$ using a Taylor series expansion,

$$
\begin{gathered}
H(s)=\sum_{n=0}^{\infty} m_{n} s^{n} \\
H(s) \approx \tilde{H}(s)=H(0)+s \frac{(H(0))^{(1)}}{1 !}+s^{2} \frac{(H(0))^{(2)}}{2 !}+\ldots .+s^{n} \frac{(H(0))^{(n)}}{n !}
\end{gathered}
$$

where $n$ is the $n^{\text {th }}$ derivative.

Simplifying, it can be rewritten as

$$
H(s) \approx \tilde{H}(s)=m_{0}+m_{1} s+m_{2} s^{2}+\ldots . .=\sum_{l=0}^{\infty} m_{n} s^{n}, m_{n}=s^{n} \frac{(H(0))^{(n)}}{n !}
$$

The $n$-th coefficient of the Taylor series expansion, $m_{n}$, of $H(s)$ is also identical to the time domain moments of the impulse response, $h(t)$. This can be seen using the Laplace transform.

The coefficients of the Taylor series expansion, $m_{n}$, are generally referred to as moments [21]. 


\subsection{Concepts of Direct Moment Matching Techniques}

Moment matching techniques, such as Asymptotic Waveform Evaluation (AWE), are based on the extraction of dominant poles and residues of a system by finding the Taylor series coefficients of the system's impulse response expanded about $s=0$ in the frequency domain [22].

Given a single input/output system, the moments, $m_{n}$, of the transfer function, $H(s)$, of order $n$, expanded about $s=0$, is

$$
H(s)=\frac{Y(s)}{X(s)}=\frac{1+a_{1} s+a_{2} s^{2}+\ldots .+a_{n} s^{n}}{b_{1} s+b_{2} s^{2}+\ldots .+b_{n} s^{n}} \approx m_{1} s+m_{2} s^{2}+\ldots .+m_{n} s^{n}
$$

To clearly illustrate the relation between the moment, poles, and residues of the system, equation 3.3.1 can be rewritten in partial fractions [23] as

$$
H(s)=\frac{k_{1}}{s-p_{1}}+\frac{k_{2}}{s-p_{2}}+\ldots+\frac{k_{n}}{s-p_{n}}+c
$$

or more concisely

$$
H(s)=c+\sum_{n=1}^{N_{p}} \frac{k_{n}}{s-\lambda_{n}}
$$

where $p_{n}$ and $k_{n}$ are the $n_{t h}$ pole residue pair, $N_{p}$ is the total number of system poles and $c$ is the direct coupling constant.

The AWE technique for direct moment matching calculates the first $2 q$ moments of the transfer function, $H(s)$, around $s=0$ in order to find the first $q$ dominant poles and residues [24]. However, techniques such as AWE that employ single point expansion, approximate the behavior of a linear or linearized system by extracting a reduced order model of size $q$ via a Padé 
approximation, which can often lead to a solution with unstable poles even for low-order approximations. In addition, AWE will become numerically unstable at higher order moment approximations due to rapid convergence to the eigenvector corresponding to the largest eigenvalue. Hence, ill-conditioning issues become a problem as moments calculated after larger values of $n$ do not add any new information to the moment-matrix and become almost identical [21], [24], [25], [26], [27], [28].

To overcome this limitation, indirect moment matching techniques based on Krylov subspace iterative methods were introduced.

\subsection{Indirect Moment Matching Techniques}

Indirect Moment Matching Techniques are based on Krylov subspace iterative methods that extract the leading eigenvalues rather than the dominant poles, see Section 3.5. This is achieved by implicitly matching the moments of the system using a different set of vectors that have the same span of the moment vectors but which are more numerically stable. Stability is achieved via a congruent transformation of a square matrix, $W$, where $A=V^{T} W V$ [23], where $V$ is square and non-singular.

A single input system with dimension $N$ is shown below

$$
\begin{aligned}
& C \dot{x}(t)+G x(t)=R u(t), \quad C, G \in \Re^{N \times N}, \quad R \in \Re^{N \times 1}, \quad l \in \Re^{N \times 1} \\
& y=l^{T} x(t)
\end{aligned}
$$

The resulting transfer function formed has the general form

$$
H(s)=l^{T}(I-s A)^{-1} B \text { where } A=-G^{-1} C, B=-G^{-1} R
$$


and the generalized eigenvalue problem from equation 3.4 .2 is

$$
\operatorname{det}[E-\lambda A]=0
$$

where the matrix $E$ is the identity matrix, $I$,

Applying a congruent transformation to equation 3.4.3 gives

$$
\operatorname{det}\left[V^{T} E V-\lambda V^{T} A V\right]=0
$$

One of the fundamental properties of a congruent transformation is that, for square nonsingular congruence transforms, the eigenvalues for the generalized symmetric eigenvalue problems, shown in equation 3.4.3, are preserved after the transform is applied as in equation 3.4.4 [29]. In other words, the poles/eigenvalues of the system represented by the transfer function, $H(s)$ shown in equation 3.4.2, are identical. As a result, the ill-conditioning problem observed by the Padé approximation is avoided, and the reduced model system can retain passivity and stability. This holds only if, for the generalized eigenvalue problem shown in equation 3.4.3, the general matrix $E$ is symmetric, non-negative definite, and the general matrix $A$ is symmetric definite.

\subsection{Krylov subspaces}

The Krylov subspace method is an example of an iterative method which is used to solve linear systems when a direct approach to finding the solution of the system becomes computationally expensive. This is most applicable in solving large sparse matrices when direct solutions, such as those using Gaussian elimination, start introducing non-zero entries into otherwise zero entries of the matrix; thereby resulting in greater data storage requirements and greater difficulty 
in solving the system in subsequent numerical manipulations.

Iterative methods work on the premise of making an initial guess of the real solution and working toward building a good approximation to the solution of the system by finding one or more leading eigenvalues of the system. This is done by replacing the original system by one that is much more easily solved [30].

The Krylov subspace method solves $A x=b$ by assuming an initial guess, $x_{0}=r$, usually with $r=b$ and performing repeated matrix-vector multiplications to obtain more accurate approximations, $x_{n}$, where $n$ is the number of iterations used [31], [32]. This subspace is denoted as $\kappa(A, r, n)$ and is useful as any vector that is a combination of the moments of the system can be expressed as a linear combination of the columns of $\kappa(A, r, n)$.

$$
\kappa(A, r, n) \equiv \operatorname{span}\left\{r, A r, A^{2} r, \ldots . A^{n-1} r\right\} \quad A \in \Re^{N \times N}, \quad x, b \in \Re^{n \times 1}
$$

\subsection{The Arnoldi Algorithm}

The Arnoldi algorithm for model reduction builds an orthonormal basis, $Q \in \Re^{N \times n}$ for $\kappa(A, r, n)$ [33] [34], and the Hessenberg matrix, $H_{n} \in \Re^{n \times n}$, related to the original matrix as illustrated in equation 3.6.1. It is basically a modified version of the Gram-Schmidt procedure for the Krylov subspace method [32].

Arnoldi [34] discovered that eigenvalues of a Hessenberg matrix formed from the matrix $A$, provide an accurate approximation to some of the eigenvalues of the original system, and developed an efficient, well-conditioned technique for approximating these eigenvalues for solving large sparse linear systems of equations, such as those that arise in finite element modeling used 
to model MEMS systems in this thesis.

The system matrix, $A$, can be used to find an upper Hessenberg matrix $H_{n}$ by the similarity transformation such that

$$
A \kappa=\kappa H_{n}
$$

where $n$ is the number of iterations and $\kappa$ is the Krylov subspace spanned in equation 3.5.1

However, the computation of $H_{n}$ involves finding the inverse of a dense matrix, so $\kappa$ and can lead to expensive computational effort and is likely to be ill-conditioned since the columns of $\kappa$ are formed based on the sequence $A^{n} r$ and become parallel to each other at larger values of $n[21]$.

In order to overcome the ill-conditioning problem, the Arnoldi algorithm replaces $K$ by taking advantage of the fact that a real matrix is orthogonal if

$$
Q^{T} Q=I
$$

where $I$ is the identity matrix.

Additionally, it is known that if the matrix is square, then $Q^{-1}=Q^{T}$, which effectively eliminates the large computational effort that would normally be required in finding the inverse.

The orthogonal matrix, $Q$, is formed such that the leading $n$ columns of $Q$ span the same space as $\kappa$ such that

$$
\kappa(A, r, n)=\operatorname{colsp}\left(\left[\begin{array}{llll}
r & A r & \ldots & A^{n-1} r
\end{array}\right]\right)=\operatorname{colsp}[Q]
$$

$Q$ is then formed by decomposing $\kappa$ using the $\mathrm{QR}$-decomposition where

$$
\kappa=Q R_{u}
$$


where $R_{u}$ is an upper triangular matrix.

Substituting equation 3.6.4 into equation 3.6.1,

$$
\begin{aligned}
& H_{n}=\kappa^{-1} A \kappa \\
& =\left(Q R_{u}\right)^{-1} A\left(Q R_{u}\right)
\end{aligned}
$$

and with some manipulation, the result is

$$
A=Q H Q^{T} \text { or } H=R_{u} H_{n} R_{u}^{-2}=Q^{T} A Q
$$

As both $R_{u}$ and $R_{u}^{-1}$ are upper triangular and $H_{n}$ is upper Hessenberg, $H$ is also upper Hessenberg.

Only the leading $n$ columns of the orthogonal matrix $Q$ are used and $n<<N$, the Hessenberg matrix formed in equation 3.6.6 has dimensions $\Re^{n \times n}$, thus reducing the original system from $\Re^{N \times N}$ to $\Re^{n \times n}$.

Another useful property of the Arnoldi algorithm is that it allows columns of $Q$ to be computed one at a time so only the required number of columns desired are formed, thereby keeping the computational effort low.

If the matrix $Q$ is decomposed into vectors as

$$
Q=\left[\begin{array}{lllll}
{\left[q_{1}\right]} & {\left[q_{2}\right]} & {\left[q_{3}\right]} & \ldots & {\left[q_{n}\right]}
\end{array}\right]
$$

Equation 3.6.6 becomes

$$
H=\left[\begin{array}{ccccc}
h_{11} & h_{12} & h_{13} & \cdots & \cdots \\
h_{21} & h_{22} & h_{23} & \cdots & \cdots \\
0 & h_{32} & h_{33} & \cdots & \cdots \\
\vdots & \vdots & \vdots & \cdots & \vdots \\
\vdots & \vdots & \vdots & \cdots & \vdots
\end{array}\right]=\left[\begin{array}{c}
{\left[q_{1}\right]} \\
{\left[q_{2}\right]} \\
{\left[q_{3}\right]} \\
\vdots \\
{\left[q_{n}\right]}
\end{array}\right] A\left[\begin{array}{lllll}
{\left[q_{1}\right]} & {\left[q_{2}\right]} & {\left[q_{3}\right]} & \cdots & {\left[q_{n}\right]}
\end{array}\right]
$$


The columns, $q_{i}$ of the orthogonal matrix have $\left\|q_{i}\right\|_{2}=1$, where $q_{i}$ is the $i$ th column of the matrix. This implies that $q_{i}^{T} q_{i}=1$ and are orthogonal to each other or $q_{i}^{T} q_{j}=0$.

This reduction of a large matrix to a small upper Hessenberg matrix using the matrix $Q$ is known as the Arnoldi alogirthm.

The Amoldi algorithm can be summarized as shown in the pseudocode outlined in Figure 3.1

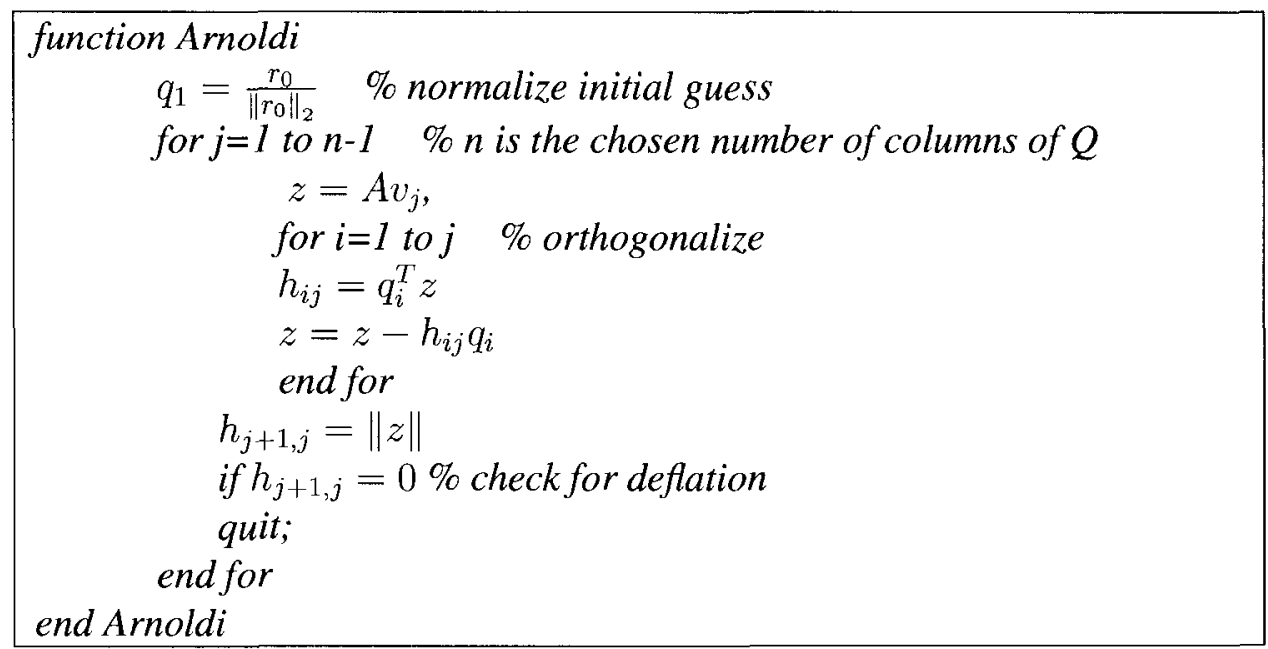

Figure 3.1: The Arnoldi Algorithm

The following describes the basic steps in the Arnoldi algorithm [35] used in the model reduction.

Step 1: The initial solution or guess, $x_{0}$, is formed by an expansion around $s=0$. For the purposes of this thesis, it is assumed that the matrices are square so all the vectors used to calculate the moments are linearly independent. The initial vector, $v_{1}$, is set to

$$
v_{1}=\frac{r_{0}}{\left\|r_{0}\right\|_{2}}
$$

where $r_{0}$ is the initial solution vector found after the deletion of the dependent vectors. 
Step 2: For $j=1 \ldots n-1$,

(a) $A v_{j}$ is computed and set to $v_{j+1}$

(b) $v_{j+1}$ is orthogonalized using a modified Gram-Schimdt algorithm

(c) Second normalization

(d) Check for deflation

(e) Go to step 2(a) and repeat without increasing $j$

At each step, the algorithm multiplies the previous Arnoldi vector $v_{j}$ by $A$ and then orthonormalizes the resulting vector $v_{j+1}$ against all previous $v$-vectors by a modified Gram-Schmidt procedure. It will stop if the vector computed in step $2(d)$ vanishes, that is if $h_{j+1, j}=0$ at iteration $j$ since this happens only if the starting vector, $r_{0}$, is a linear combination of $j$ eigenvectors of $A$, which is a result of the moments of the reduced-system being identical to those of the original system [36].

A disadvantage of the Arnoldi algorithm is that each new Arnoldi vector must be orthogonal to all previously generated vectors, so the computational cost grows with the dimension of the subspace [37].

\subsection{Model Reduction of Second Order Systems via Arnoldi}

For the purposes of this thesis, systems of higher order are modeled with the following secondorder differential equation

$$
\sum_{N}:\left\{\begin{array}{c}
M \ddot{z}(t)+D \dot{z}(t)+K z(t)=F u(t) \\
y(t)=l^{T} z(t)
\end{array}\right.
$$


given that $z(0)=z_{0}$ and $\dot{z}(0)=\dot{z}_{0}$

with $t=$ time variable,

$N=$ state-space dimension, $x(t) \in \Re^{N}$, with $m$ inputs and $p$ outputs

$u(t), y(t)$ are the input force and response respectively

$M, D, K$ are the system matrices i.e. Mass, Damping, and Stiffness matrices

For $D=0$, the system is a purely oscillatory i.e. undamped.

As the Arnoldi algorithm is designed for first-order systems, equation 3.7.1 is linearized into a first-order companion form by making $v=\frac{d z}{d t}$ and rewriting the equation to be

$$
\begin{aligned}
& {\left[\begin{array}{cc}
M & D \\
0 & I
\end{array}\right]\left[\begin{array}{l}
\dot{v} \\
v
\end{array}\right]+\left[\begin{array}{cc}
0 & K \\
-I & 0
\end{array}\right]\left[\begin{array}{l}
v \\
z
\end{array}\right]=\left[\begin{array}{l}
F \\
0
\end{array}\right]} \\
& y=l^{T} z(t)
\end{aligned}
$$

where $\mathrm{I}$ is an $N \times N$ identity matrix

If

$$
C=\left[\begin{array}{cc}
M & D \\
0 & I
\end{array}\right], G=\left[\begin{array}{cc}
0 & K \\
0 & -I
\end{array}\right], R=\left[\begin{array}{c}
F \\
0
\end{array}\right], x(t)=\left[\begin{array}{l}
v \\
z
\end{array}\right]
$$

Equation 3.7 .2 can be rewritten in the form

$$
\begin{aligned}
& C \dot{x}(t)+G x(t)=R u(t) \\
& y=l^{T} x(t)
\end{aligned}
$$

where $C, G \in \Re^{N \times N}, R \in \Re^{N \times 1}, L \in \Re$, and $N$ is the total number of state space variables. Equation 3.7.4 is now in the first-order form in which the Arnoldi algorithm can be implemented.

By multiplying both sides by $G^{-1}$ and rearranging, equation 3.7 .4 becomes 


$$
\begin{gathered}
A \dot{x}(t)=x(t)-B u(t) \\
y(t)=l^{T} x(t)
\end{gathered} \quad, \quad A=-G^{-1} C, \quad B=-G^{-1} R
$$

Taking the Laplace transform of equation 3.7.5

$$
\begin{aligned}
& s A X(s)=X(s)-B U(s) \\
& Y(s)=l^{T} X(s)
\end{aligned}
$$

Rearranging equation 3.7.6 results in the transfer function of the form

$$
H(s)=\frac{Y(s)}{X(s)}=l^{T}(I-s A)^{-1} B
$$

To obtain the reduced model of the original system using the Arnoldi algorithm, a change of variables is applied by mapping the original system variable, $x$ of dimension $N$, to the smaller vector, $\hat{x}$, using the orthogonal matrix generated by the Arnoldi Algorithm, $Q,[21]$ such that

$$
x=Q \hat{x}
$$

Applying this mapping to the original system equations, the reduced model of order $\Re^{q \times q}$ is achieved. This can be represented as

$$
\hat{H}(s)=\frac{Y(s)}{X(s)}=l^{T} Q\left(I-s Q^{T} A Q\right)^{-1} Q^{T} R=L^{T} Q(I-s H)^{-1} Q^{T} R
$$

A comparison of the original transfer function, $Y(s)$, and the reduced transfer function, $\hat{Y}(s)$, shows that the eigenvalues of $H$ are the eigenvalues of the reduced system and represent a good approximation of the leading eigenvalues of $A$ and, hence, the poles of the original transfer function [21], [38]. 


\subsection{The Second Order Arnoldi (SOAR) Algorithm}

The Arnoldi algorithm requires systems represented by second-order differential equations to be reformulated by folding the original system into first-order resulting in the loss of the secondorder form. In contrast, the SOAR procedure computes an orthonormal basis of the secondorder Krylov subspace directly by choosing a subspace which the full order system is projected onto.

This direct projection takes advantage of the linearized structure of the matrix as follows [18], [39], [40], [2]:

A general second-order system has the form

$$
\begin{aligned}
& M \ddot{z}(t)+D \dot{z}(t)+K z(t)=F u(t) \\
& y(t)=l^{T} z(t)
\end{aligned}
$$

This second-order system can be rewritten into the first-order system

$$
\begin{aligned}
& C \dot{x}(t)+G x(t)=\hat{b} u(t) \\
& y(t)=\hat{l}^{T} x(t)
\end{aligned}
$$

where

$$
C=\left[\begin{array}{cc}
D & M \\
-I & 0
\end{array}\right], G=\left[\begin{array}{cc}
K & 0 \\
0 & I
\end{array}\right], R=\left[\begin{array}{c}
F \\
0
\end{array}\right], x(t)=\left[\begin{array}{c}
v(t) \\
\dot{v}(t)
\end{array}\right], \hat{l}=\left[\begin{array}{l}
l \\
0
\end{array}\right]
$$

The transfer function of the system given in equation 3.8 .1 is

$$
H(s)=l^{T}\left(s^{2} M+s D+K\right)^{-1} F
$$

and the equivalent transfer function for equation 3.8.2 is

$$
H(s)=l^{T}(s C+G)^{-1} F
$$


Re-arranging equation 3.8 .4 as

$$
H(s)=l^{T}\left(I+s G^{-1} C\right)^{-1} B=\hat{l}^{T}(I-s A)^{-1} B
$$

where $A=-G^{-1} C$ and $B=G^{-1} F$

Expanding the matrix $A$ gives

$$
A=-G^{-1} C=\left[\begin{array}{cc}
-K^{-1} D & -K^{-1} M \\
I & 0
\end{array}\right]:=\left[\begin{array}{cc}
\hat{A} & \hat{B} \\
I & 0
\end{array}\right]
$$

where $\hat{A}=-K^{-1} D$ and $\hat{B}=-K^{-1} M$

The second-order Krylov subspace can then be stated as

$$
\begin{aligned}
& \kappa(A, B, n)=\kappa\left(G^{-1} C, G^{-1} F, n\right) \\
& \kappa(A, B, n)=\operatorname{span}\left\{G^{-1} F, \quad\left(G^{-1} C\right) G^{-1} F, \quad\left(G^{-1} C\right)^{2} G^{-1} F, \quad \cdots \quad,\left(G^{-1} C\right)^{n-1} G^{-1} F\right\}
\end{aligned}
$$

If $r_{n}$ is denoted as follows

$$
\begin{aligned}
& r_{n}=\left[\begin{array}{l}
r_{n}^{d} \\
r_{n}^{v}
\end{array}\right]=\left(G^{-1} C\right) B=\left(G^{-1} C\right)\left[\begin{array}{c}
r_{n-1}^{d} \\
r_{n-1}^{v}
\end{array}\right] \\
& r_{n}=\left[\begin{array}{cc}
K^{-1} D & K^{-1} M \\
-I & 0
\end{array}\right]\left[\begin{array}{l}
r_{n-1}^{d} \\
r_{n-1}^{v}
\end{array}\right]=\left[\begin{array}{c}
K^{-1} D r_{n-1}^{d}+K^{-1} M r_{n-1}^{v} \\
-r_{n-1}^{d}
\end{array}\right]
\end{aligned}
$$

It is observed that the $r_{n}^{d}$ is the vector corresponding to the displacement portion and $r_{n}^{v}$ is the vector corresponding to the velocity portion of the vector $r_{n}$ respectively. This implies that the $n t h$ velocity portion of the $r_{n}^{v}$ vector is the $(n-1)-t h$ displacement of the $r_{n-1}^{d}$. In other words the velocity portion, $r_{n}^{v}$, of $r_{n}$ is one step-delay of the first portion $r_{n-1}^{d}$ of $r_{n-1}$.

In addition, let

$$
\begin{aligned}
& r_{0}=K^{-1} F \\
& r_{1}=\hat{A} r_{0} \\
& r_{n}=\hat{A} r_{n-1}+r_{0}+\hat{B} r_{n-2} \text { for } n \geq 2
\end{aligned}
$$


where $\left\{r_{n}\right\}$ is the second-order Krylov vector sequence.

The subspace spanned by this sequence is called the second-order Krylov subspace, $\left\{\hat{A}^{n-1}, r_{0}\right\}$. The projection subspace spanned by $\left\{r_{n}\right\}$ of $\Re^{N}$ should be able to provide sufficient information for dimension reduction as it does for the Krylov subspace, $\kappa\left\{\hat{A}, \hat{B}, r_{0}, n\right\}$ of $\Re^{2 N}$ [36], [41].

Formally, an $n t h$ second-order Krylov subspace with matrices $A$ and $B$ and starting vector, $r_{0}$ is defined by [42] as

$$
\kappa\left(\hat{A}, \hat{B}, r_{0}, n\right)=\operatorname{span}\left\{r_{0}, r_{1}, \ldots . . r_{n-1}\right\}
$$

The SOAR procedure can be summarized as shown in Figure 3.2.

The main difference between SOAR and the Arnoldi algorithm is that, in SOAR, the nonzero elements of $t_{i j}$ of the $(n+1) \times n$ upper Hessenberg matrix, $\hat{H}_{n}$, are chosen to enforce the orthonormality of the vectors $q_{j}$ of dimensions $N$, but in the Arnoldi algorithm, the nonzero elements, $h_{i j}$ of the $(n+1) \times n$ upper Hessenberg matrix, $\hat{H}_{n}$, are determined to ensure orthonormality of the vectors $\left\{v_{j}\right\}$ of dimension $2 N$ [40], [42], [43].

Also, using the second-order Krylov subspace method, SOAR, as the projection method, the reduced system will not only match the same number of moments as the one derived based on the projection on the Krylov subspace, $\kappa_{n}$, it will also preserve the second-order forms of the original system $\sum_{N}$. 


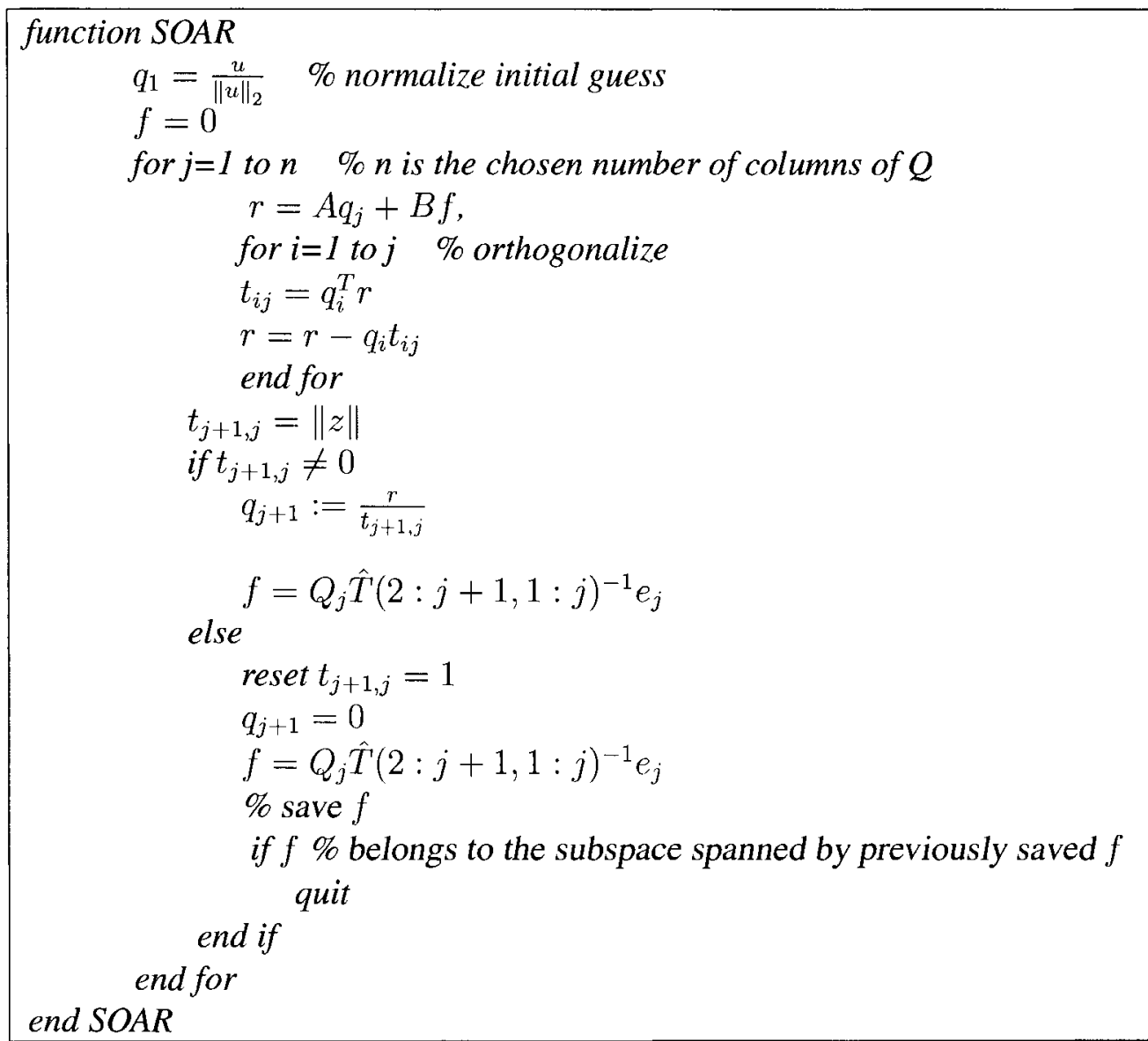

Figure 3.2: The SOAR procedure

\subsection{Model Reduction of Second Order Systems via SOAR}

Recall that the second-order differential equation being considered in this thesis is

$$
\sum_{N}:\left\{\begin{array}{c}
M \ddot{z}(t)+D \dot{z}(t)+K z(t)=F u(t) \\
y(t)=L z(t)
\end{array}\right.
$$

given that $z(0)=z_{0}, \dot{z}(0)=\dot{z}_{0}$

with $t=$ time, $z(t) \in \Re^{N}$,

$N=$ state space dimension,

$z(t), y(t)$ are the input force and output measurement respectively

$M, D, K \in \Re^{N \times N}$ are system matrices i.e. Mass, Damping, and Stiffness matrices 
Taking the Laplace Transform of equation 3.9.1,

$$
\left\{\begin{array}{c}
s^{2} M Z(s)+s D Z(s)+K Z(s)=F U(s) \\
Y(s)=L Z(s)
\end{array}\right.
$$

where $Z(s), Y(s)$, and $U(s)$ represent the Laplace Transform of $z(t), y(t)$, and $u(t)$ respectively.

By eliminating $Z(s)$ in equation 3.9.2 the transfer function of the system, $H(s)$, is found below as

$$
H(s)=l^{T}\left(s^{2} M+s D+K\right)^{-1} F
$$

The transfer function $H(s)$ can be written in the linearized form as follows

$$
H(s)=\hat{l}^{T}(s C+G)^{-1} \hat{F}
$$

where

$$
C=\left[\begin{array}{cc}
D & M \\
-I & 0
\end{array}\right] \quad G=\left[\begin{array}{cc}
K & 0 \\
0 & I
\end{array}\right] \quad \hat{F}=\left[\begin{array}{l}
F \\
0
\end{array}\right] \quad \hat{l}=\left[\begin{array}{l}
l \\
0
\end{array}\right]
$$

As with the Arnoldi algorithm, the reduced model can be expressed by a simple change of state variables, where the state vector, $x(t)$, of the original system given in equation 3.9 .1 , is substituted by another state vector, $z(t)$, constrained to the subspace, $G$, and spanned by the orthogonal matrix, $Q$, as follows:

$$
x(t)=Q_{n} z(t)
$$

Substituting equation 3.9 .6 and using the same principal of orthogonality as the Arnoldi algorithm, where a real matrix is orthogonal if $Q^{T} Q=I$ and, if the matrix is square then 
$Q^{-1}=Q^{T}$, the reduced matrices of size $\Re^{(n+1) \times n}$, where $n$ is the desired size of the subspace, can be written as

$$
\sum_{n}:\left\{\begin{array}{c}
Q^{T} M Q \ddot{z}(t)+Q^{T} D Q \dot{z}(t)+Q^{T} K Q z(t)=Q^{T} F u(t) \\
Q^{T} y(t)=Q^{T} l^{T} Q z(t)
\end{array}\right.
$$

or more simply as

$$
\sum_{n}:\left\{\begin{array}{c}
M_{r e d} \ddot{z}(t)+D_{r e d} \dot{z}(t)+K_{r e d} z(t)=F_{r e d} u(t) \\
y_{\text {red }}(t)=l_{\text {red }} z(t)
\end{array}\right.
$$

where $M_{r e d}=Q^{T} M Q, D_{\text {red }}=Q^{T} D Q, K_{\text {red }}=Q^{T} K Q, F_{r e d}=Q^{T} F, y_{\text {red }}=Q^{T} y$, and $l_{\text {red }}=Q^{T} l^{T}$

This change of state variables can also be applied to the transfer function in equation 3.9.6, and $H(s)$ restated as

$$
H_{r e d}(s)=l_{r e d}^{T}\left(s^{2} M_{r e d}+s D_{r e d}+K_{r e d}\right)^{-1} F_{r e d}=l_{r e d}^{T}\left(s C_{r e d}+G_{r e d}\right)^{-1} b_{r e d}
$$

where

$$
C_{r e d}=\left[\begin{array}{cc}
D_{r e d} & M_{r e d} \\
-I & 0
\end{array}\right] \quad \text { and } \quad G_{r e d}=\left[\begin{array}{cc}
K_{r e d} & 0 \\
-I & 0
\end{array}\right]
$$

Furthermore, $C_{r e d}$ and $G_{r e d}$ can also be expressed as

$$
C_{r e d}=Q^{T} C Q \text { and } G_{r c d}=Q^{T} G Q
$$

which in turn implies that the moments of the system can be written as

$$
m_{l}^{[n]}=l_{r e d}^{T}\left(-G_{r e d}^{-1} C_{r e d}\right)^{l}\left(G_{r e d}^{-1} F_{r e d}\right) \quad \text { for } \quad l \geq 0
$$


Expanding about the point, $s_{0}$,

$$
H(s)=l^{T}\left(\left(s-s_{0}\right)^{2} M+\left(s-s_{0}\right) \tilde{D}+\tilde{K}\right)^{-1} F
$$

where $\tilde{D}=2 s_{0} M+D$, and $\tilde{K}=2 s_{0}^{2} M+s_{0} D+K$ and $s_{0}$ is an arbitrary but fixed value such that the matrix, $\tilde{K}$, is non-singular. $\tilde{D}, \tilde{K}$ and the expansion point, $s_{0}$, form the basis for the initial guess for the second-order Krylov subspace projection.

By applying the SOAR procedure, the orthonormal basis, $Q$, for the second-order Krylov subspace, $G\left(A, B ; r_{0}\right)$ can be generated with the matrices $A=-K^{-1} \tilde{D}$ and $B=-K^{-1} \tilde{M}$ and $r_{0}=K^{-1} b$ about the expansion point $s_{0}$.

The reduced transfer function in equation 3.9.13 is then

$$
H_{r e d}(s)=l_{r e d}^{T}\left(\left(s-s_{0}\right)^{2} M_{r e d}+\left(s-s_{0}\right) \tilde{D}_{r e d}+\tilde{K}_{r e d}\right)^{-1} F_{r e d}
$$

where $M_{r e d}=Q^{T} M Q, \tilde{D}_{r e d}=Q^{T} D Q, \tilde{K}_{r e d}=Q^{T} K Q, F_{r e d}=Q^{T} b, y_{\text {red }}=Q^{T} y$, and $l_{r e d}=Q^{T} l^{T}$

$H_{\text {red }}$ can be manipulated algebraically to be expressed in the desired, simplified form for the reduced transfer function as

$$
H_{r e d}(s)=l_{r e d}^{T}\left(s^{2} M_{r e d}+s D_{r e d}+K_{r e d}\right)^{-1} F_{r e d}
$$

where $M_{r e d}=Q^{T} M Q, D_{r e d}=Q^{T} D Q, K_{r e d}=Q^{T} K Q, F_{r e d}=Q^{T} b, y_{r e d}=Q^{T} y$, and $l_{\text {red }}=Q^{T} l^{T}$

The transformed matrix triplet, $(M, \tilde{D}, \tilde{K})$ is used to generate an orthonormal basis, $Q$ of the second-order projection subspace, $G,[40]$. 


\subsection{Conclusion}

From a theoretical standpoint, model reduction techniques of second-order systems projected into the second-order Krylov subspace have many advantages over model reduction based on the Arnoldi algorithm which requires the second-order system to be reformulated to a first-order companion form. This, in itself, is where the disadvantages of Arnoldi arise, as the structural properties and characteristics inherent to a second-order system are lost. The most obvious of these being the loss of symmetry of the sparse matrices. Although the companion form can be formulated to retain symmetry, the resulting matrices often need to be scaled to avoid other issues such as ill-conditioning problems, and the consequent loss of stability and passivity. Another disadvantage of the Arnoldi implementation for model reduction lies in the fact that the companion form results in an orthogonal matrix which is expectedly dense but also $2 N \times n$ the size, compared to the SOAR implementation which results in the an orthogonal matrix of $(N \times(n+1)$ dimension, where $n$ is the desired number of moments. This can become a problem if the size of the system to be modeled is very large, and size limitations can also be a consequence of the Arnoldi algorithm.

However, these disadvantages are based on the theoretical analysis of the two model reduction techniques, so a thorough numerical analysis will be performed in Chapter 4 to compare the accuracy and advantages of either system. This analysis will take three cantilevers modeled with FEM and will compare the number of moments taken to generate the reduced model and the level of accuracy each achieves compared to the full or real model. The same will be done 
for two models implemented using SUGAR modeling. In addition, all the models will be realized with different amounts of damping to examine what effect increasing complexity has on the outlined model reduction techniques. 


\section{Chapter 4}

\section{Stability and Passivity of Model Reduction}

When performing model reduction techniques, two of the most important properties of the original, full system that must be preserved are stability and passivity of the original system.

For the generalized state-space, single-input/single-output system defined as

$$
\begin{aligned}
& E \dot{x}(t)=A x(t)+B u(t) \\
& y=l^{T} x(t)
\end{aligned}
$$

with the transfer function is defined as

$$
H(s)=l^{T}(s E-A)^{-1} B
$$

where $E, A \in \Re^{N \times N}, B \in \Re^{N \times m}, l \in \Re^{p \times N}, u \in \Re^{m}, y \in \Re^{p}$, and $x \in \Re^{N}$. In this case, $p=m=1$.

For a system to be stable, control theory states that the complex eigenvalues of the resulting matrix, $E^{-1} A$, must have strictly negative real parts. However, stability does not guarantee passivity [44].

Passivity, in engineering applications, refers to systems or components that consume, but do not produce energy so that they are incapable of power gain. It is highly desirable for modeled 
passive systems to have reduced models that are also passive in order to avoid non-physical behaviors which can cause unstable time-domain behaviors due to the loss of passivity if further applications and simulations of the reduced system are performed [45].

Model reduction techniques that use direct moment matching based on a Padé approximation can be unstable as a result of ill-conditioning issues associated directly with the Padé approximation. However, even the accepted solution to overcoming these issues, indirect moment matching techniques based on Krylov subspace methods for model reduction, cannot guarantee stability and passivity of the reduced models.

Recapitulating, Krylov methods perform model reduction through a congruent transformation which preserves the positive-realness of the system matrices that are internal to the statespace representation. While this is useful in RLC circuit simulations, it cannot be practically extended to other kinds of systems without facing pitfalls. The most prominent being that it is the input-output transfer function of the system that determines whether or not the state-space model represents a passive system, not the internal representation of the model. Also, in the reformulation of second-order systems to first-order, the algorithms implemented for model reduction, such as the Arnoldi algorithm, can result in asymmetric and non-positive semidefinite system matrices. The loss of such important physical properties means that a change of basis by congruent transformation may be impossible and can lead to loss of sparsity of the model and, hence, become completely impractical to implement for large-scale systems [45].

To further illustrate this point, an examination of Krylov methods that call for the implementation of the model reduction of a second-order system via a reformulation to a first-order system and the conditions that should be met to ensure the stability and passivity of the reduced 
model will be performed here.

For the system defined by the equations given in 4.0 .1 , the conditions that should guarantee stability and passivity according to [20], [35], [46], [47], [44], [48], [49], [50] are outlined below.

Firstly, any linear dynamic system is passive if, and only if its transfer function is positive real.

For positive realness this implies that

1. $H(s)$ is analytic for $\operatorname{Re}(s)>0$, i.e. there are no poles in the right half complex plane,

2. $H(\bar{s})=\overline{H(s)}$ for all $s \in \mathbb{C}$ where $\mathbb{C}$ is the complex plane,

3. $H(s)+(H(s))^{H} \geq 0$ for all $s>\mathbb{C}_{+}$

4. $\operatorname{Re}\left(w^{H} H(s) w\right) \geq 0$ for all $s \in C$ with $\operatorname{Re}(s)>0$ and $w \in \mathbb{C}^{m}$

where $\overline{(.)}$ denotes the conjugate complex and $(.)^{H}$ denotes the hermitian which is the complex conjugate of the transpose of a complex matrix.

Lemma 3.1 If $A+A^{T} \preceq 0, E=E^{T} \succeq 0$ and $l=B^{T}$, then the corresponding transfer function, $H(s)$, given in equation 4.0.2, is stable and positive real. More specifically, if the state-space model is symmetric, that is $A=A^{T}, B=l^{T}$, and $A$ is negative semidefinite so that $\operatorname{Re}\{H(s)\}=\operatorname{Re}\{s I-A\}=-\frac{1}{2}\left(A+A^{T}\right)=-A \preceq 0$, the system is positive real [51], [52].

For the detailed proof of this, see [48], [53], [54], [55], [56]. If the conditions outlined in Lemma 3.1 are satisfied, then the system is passive. As noted earlier, passivity, as defined 
by these conditions, is a property of the transfer function not its realization, so a system can still be passive and the conditions stated here not necessarily true. However, since passivity guarantees stability, if the system is passive it must be stable, so the conditions for stability are as follows [35],

Corollary 3.1 The system is stable if $A+A^{T} \preceq 0, E=E^{T} \succeq 0$.

Lemma 3.2 If a general matrix, $W$, is symmetric and $W \in \Re^{N \times N}, W \geq 0$ signifies that $W$ is positive (negative) definite. If this is true for $W$ and there exists a matrix $V$ such that $V \in \Re^{N \times N}$ is a full rank matrix, then $V^{T} W V$ is positive (negative) definite. This is also true for when $W$ is semi-definite [35].

Lemma 3.3 The second-order system in equation 4.0.3 will have no poles in the right half of the complex plane if the system matrices, $M, D$, and $K$ are symmetric, that is if $M=$ $M^{T} \succeq 0, D=D^{T} \succeq 0, K=K^{T} \succeq 0$ and, hence, will be stable [35], [57].

Recall, the system being considered in this thesis has the general second-order form

$$
\left\{\begin{array}{c}
M \ddot{z}(t)+D \dot{z}(t)+K z(t)=F u(t) \\
y(t)=l^{T} z(t)
\end{array}\right.
$$

where $M, D$, and $K$ are the mass, damping, and stiffness matrices respectively.

Also, for the implementation of model reduction of such systems via the Arnoldi algorithm, the second-order differential equation represented in equation 4.0.3 has to be folded into a firstorder system, as given in equation 4.0 .2 , by the substitution of $v(t)=\dot{z}(t)$.

This results in system matrices $E$ and $A$ having the form 


$$
\left\{\begin{array}{l}
\underbrace{\left[\begin{array}{cc}
-W & 0 \\
0 & M
\end{array}\right]}_{E} \underbrace{\left[\begin{array}{c}
\dot{z}(t) \\
\ddot{z}(t)
\end{array}\right]}_{\dot{x}}=\underbrace{\left[\begin{array}{cc}
0 & W \\
-K & -D
\end{array}\right]}_{A} \underbrace{\left[\begin{array}{c}
z(t) \\
\dot{z}(t)
\end{array}\right]}_{x}+\underbrace{\left[\begin{array}{c}
0 \\
F(t)
\end{array}\right]}_{B} u(t) \\
y(t)=\underbrace{\left[\begin{array}{ll}
L & 0
\end{array}\right]}_{C} \underbrace{\left[\begin{array}{c}
z(t) \\
\dot{z}(t)
\end{array}\right]}_{x}
\end{array}\right.
$$

According to [35], [57], [58], if $K, M$ and $D$ are symmetric and positive definite, as is the case in mechanical systems, the symmetry and positive definiteness of these matrices are automatically transferred to the first-order matrices in equation 4.0.1, $E$ and $A$. This implies that if $M$ and $-K$ are symmetric and positive definite or positive semi-definite and $-D$ is symmetric, as discussed in Lemma 3.2, then $A$ is symmetric and $E$ is symmetric and positive definite or positive semi-definite.

To further ensure symmetry the formulation of $E$ and $A$ can be constructed to retain symmetry when creating the first-order system matrices, as illustrated in equation 4.0 .4 with $W=-M$, if $M, D$, and $K$ are all symmetric and $M$ is non-singular. However, the more common choice found in most implementations of the Arnoldi algorithm for second-order systems is usually $W=I[57]$.

These observations suggest that the formulation of the first-order system does not require any special considerations, since once the conditions outlined above are met, the corresponding reduced model should also be stable and passive.

However, in order to conclusively illustrate the superiority of one type of model reduction over the other, in this case the comparison between the Arnoldi algorithm and the SOAR procedure, a complete analysis of the reformulation from second-order to first-order was performed. 
This was done by collapsing the second-order differential system, described in equation 4.0.3 into the first-order system with the general form as in equation 4.0.1 in sixteen different configurations.

While some of these configurations may seem mathematically inconsequential, for the sake of completeness, they were still considered and were eventually shown to be more as important than initially thought.

\subsection{Testing Stability and Passivity}

The sixteen observable configurations for the equivalent first-order system are as follows

$$
\underbrace{\left[\begin{array}{cc}
D & M \\
-I & 0
\end{array}\right]}_{E}\left[\begin{array}{c}
\dot{z}(t) \\
\ddot{z}(t)
\end{array}\right]+\underbrace{\left[\begin{array}{cc}
K & 0 \\
0 & I
\end{array}\right]}_{A}\left[\begin{array}{c}
z(t) \\
\dot{z}(t)
\end{array}\right]=\underbrace{\left[\begin{array}{c}
F(t) \\
0
\end{array}\right]}_{B}, y(t)=\left[\begin{array}{ll}
L & 0
\end{array}\right]\left[\begin{array}{c}
z(t) \\
\dot{z}(t)
\end{array}\right]
$$

$$
\underbrace{\left[\begin{array}{cc}
D & M \\
I & 0
\end{array}\right]}_{E}\left[\begin{array}{c}
\dot{z}(t) \\
\ddot{z}(t)
\end{array}\right]+\underbrace{\left[\begin{array}{cc}
K & 0 \\
0 & -I
\end{array}\right]}_{A}\left[\begin{array}{c}
z(t) \\
\dot{z}(t)
\end{array}\right]=\underbrace{\left[\begin{array}{c}
F(t) \\
0
\end{array}\right]}_{B}, y(t)=\left[\begin{array}{ll}
L & 0
\end{array}\right]\left[\begin{array}{c}
z(t) \\
\dot{z}(t)
\end{array}\right]
$$

$$
\underbrace{\left[\begin{array}{cc}
0 & M \\
-I & 0
\end{array}\right]}_{E}\left[\begin{array}{c}
\dot{z}(t) \\
\ddot{z}(t)
\end{array}\right]+\underbrace{\left[\begin{array}{cc}
K & D \\
0 & I
\end{array}\right]}_{A}\left[\begin{array}{c}
z(t) \\
\dot{z}(t)
\end{array}\right]=\underbrace{\left[\begin{array}{c}
F(t) \\
0
\end{array}\right]}_{B}, y(t)=\left[\begin{array}{ll}
L & 0
\end{array}\right]\left[\begin{array}{c}
z(t) \\
\dot{z}(t)
\end{array}\right]
$$

$$
\underbrace{\left[\begin{array}{cc}
0 & M \\
I & 0
\end{array}\right]}_{E}\left[\begin{array}{c}
\dot{z}(t) \\
\ddot{z}(t)
\end{array}\right]+\underbrace{\left[\begin{array}{cc}
K & D \\
0 & -I
\end{array}\right]}_{A}\left[\begin{array}{c}
z(t) \\
\dot{z}(t)
\end{array}\right]=\underbrace{\left[\begin{array}{c}
F(t) \\
0
\end{array}\right]}_{B}, y(t)=\left[\begin{array}{ll}
L & 0
\end{array}\right]\left[\begin{array}{c}
z(t) \\
\dot{z}(t)
\end{array}\right]
$$




$$
\underbrace{\left[\begin{array}{cc}
M & D \\
0 & -I
\end{array}\right]}_{E}\left[\begin{array}{c}
\ddot{z}(t) \\
\dot{z}(t)
\end{array}\right]+\underbrace{\left[\begin{array}{cc}
0 & K \\
I & 0
\end{array}\right]}_{A}\left[\begin{array}{c}
\dot{z}(t) \\
z(t)
\end{array}\right]=\underbrace{\left[\begin{array}{c}
F(t) \\
0
\end{array}\right]}_{B}, y(t)=\left[\begin{array}{ll}
0 & L
\end{array}\right]\left[\begin{array}{c}
\dot{z}(t) \\
z(t)
\end{array}\right]
$$

$$
\underbrace{\left[\begin{array}{cc}
M & D \\
0 & I
\end{array}\right]}_{E}\left[\begin{array}{c}
\ddot{z}(t) \\
\dot{z}(t)
\end{array}\right]+\underbrace{\left[\begin{array}{cc}
0 & K \\
-I & 0
\end{array}\right]}_{A}\left[\begin{array}{c}
\dot{z}(t) \\
z(t)
\end{array}\right]=\underbrace{\left[\begin{array}{c}
F(t) \\
0
\end{array}\right]}_{B}, y(t)=\left[\begin{array}{ll}
0 & L
\end{array}\right]\left[\begin{array}{c}
\dot{z}(t) \\
z(t)
\end{array}\right]
$$

$$
\underbrace{\left[\begin{array}{cc}
M & 0 \\
0 & I
\end{array}\right]}_{E}\left[\begin{array}{c}
\ddot{z}(t) \\
\dot{z}(t)
\end{array}\right]+\underbrace{\left[\begin{array}{cc}
D & K \\
-I & 0
\end{array}\right]}_{A}\left[\begin{array}{c}
\dot{z}(t) \\
z(t)
\end{array}\right]=\underbrace{\left[\begin{array}{c}
F(t) \\
0
\end{array}\right]}_{B}, y(t)=\left[\begin{array}{ll}
0 & L
\end{array}\right]\left[\begin{array}{c}
\dot{z}(t) \\
z(t)
\end{array}\right]
$$

$$
\underbrace{\left[\begin{array}{cc}
M & 0 \\
0 & -I
\end{array}\right]}_{E}\left[\begin{array}{c}
\ddot{z}(t) \\
\dot{z}(t)
\end{array}\right]+\underbrace{\left[\begin{array}{cc}
D & K \\
I & 0
\end{array}\right]}_{A}\left[\begin{array}{c}
\dot{z}(t) \\
z(t)
\end{array}\right]=\underbrace{\left[\begin{array}{c}
F(t) \\
0
\end{array}\right]}_{B}, y(t)=\left[\begin{array}{ll}
0 & L
\end{array}\right]\left[\begin{array}{c}
\dot{z}(t) \\
z(t)
\end{array}\right]
$$

$$
\underbrace{\left[\begin{array}{cc}
I & 0 \\
D & M
\end{array}\right]}_{E}\left[\begin{array}{c}
\dot{z}(t) \\
\ddot{z}(t)
\end{array}\right]+\underbrace{\left[\begin{array}{cc}
0 & -I \\
K & 0
\end{array}\right]}_{A}\left[\begin{array}{c}
z(t) \\
\dot{z}(t)
\end{array}\right]=\underbrace{\left[\begin{array}{c}
0 \\
F(t)
\end{array}\right]}_{B}, y(t)=\left[\begin{array}{ll}
L & 0
\end{array}\right]\left[\begin{array}{c}
z(t) \\
\dot{z}(t)
\end{array}\right]
$$

$$
\underbrace{\left[\begin{array}{cc}
-I & 0 \\
D & M
\end{array}\right]}_{E}\left[\begin{array}{c}
\dot{z}(t) \\
\ddot{z}(t)
\end{array}\right]+\underbrace{\left[\begin{array}{cc}
0 & I \\
K & 0
\end{array}\right]}_{A}\left[\begin{array}{c}
z(\ell) \\
\dot{z}(t)
\end{array}\right]=\underbrace{\left[\begin{array}{c}
0 \\
F(t)
\end{array}\right]}_{B}, y(t)=\left[\begin{array}{ll}
L & 0
\end{array}\right]\left[\begin{array}{c}
z(\ell) \\
\dot{z}(t)
\end{array}\right]
$$




$$
\underbrace{\left[\begin{array}{cc}
-I & 0 \\
0 & M
\end{array}\right]}_{E}\left[\begin{array}{c}
\dot{z}(t) \\
\ddot{z}(t)
\end{array}\right]+\underbrace{\left[\begin{array}{cc}
0 & I \\
K & D
\end{array}\right]}_{A}\left[\begin{array}{c}
z(t) \\
\dot{z}(t)
\end{array}\right]=\underbrace{\left[\begin{array}{c}
0 \\
F(t)
\end{array}\right]}_{B}, y(t)=\left[\begin{array}{ll}
L & 0
\end{array}\right]\left[\begin{array}{c}
z(t) \\
\dot{z}(t)
\end{array}\right]
$$

$$
\underbrace{\left[\begin{array}{cc}
I & 0 \\
0 & M
\end{array}\right]}_{E}\left[\begin{array}{c}
\dot{z}(t) \\
\ddot{z}(t)
\end{array}\right]+\underbrace{\left[\begin{array}{cc}
0 & -I \\
K & D
\end{array}\right]}_{A}\left[\begin{array}{c}
z(t) \\
\dot{z}(t)
\end{array}\right]=\underbrace{\left[\begin{array}{c}
0 \\
F(t)
\end{array}\right]}_{B}, y(t)=\left[\begin{array}{ll}
L & 0
\end{array}\right]\left[\begin{array}{c}
z(t) \\
\dot{z}(t)
\end{array}\right]
$$

$$
\underbrace{\left[\begin{array}{cc}
0 & I \\
M & D
\end{array}\right]}_{E}\left[\begin{array}{c}
\ddot{z}(t) \\
\dot{z}(t)
\end{array}\right]+\underbrace{\left[\begin{array}{cc}
-I & 0 \\
0 & K
\end{array}\right]}_{A}\left[\begin{array}{c}
\dot{z}(t) \\
z(t)
\end{array}\right]=\underbrace{\left[\begin{array}{c}
0 \\
F(t)
\end{array}\right]}_{B}, y(t)=\left[\begin{array}{ll}
0 & L
\end{array}\right]\left[\begin{array}{c}
\dot{z}(t) \\
z(t)
\end{array}\right]
$$

$$
\underbrace{\left[\begin{array}{cc}
0 & -I \\
M & D
\end{array}\right]}_{E}\left[\begin{array}{c}
\ddot{z}(t) \\
\dot{z}(t)
\end{array}\right]+\underbrace{\left[\begin{array}{cc}
I & 0 \\
0 & K
\end{array}\right]}_{A}\left[\begin{array}{c}
\dot{z}(t) \\
z(t)
\end{array}\right]=\underbrace{\left[\begin{array}{c}
0 \\
F(t)
\end{array}\right]}_{B}, y(t)=\left[\begin{array}{ll}
0 & L
\end{array}\right]\left[\begin{array}{c}
\dot{z}(t) \\
z(t)
\end{array}\right]
$$

$$
\underbrace{\left[\begin{array}{cc}
0 & -I \\
M & 0
\end{array}\right]}_{E}\left[\begin{array}{c}
\ddot{z}(t) \\
\dot{z}(t)
\end{array}\right]+\underbrace{\left[\begin{array}{cc}
I & 0 \\
D & K
\end{array}\right]}_{A}\left[\begin{array}{c}
\dot{z}(t) \\
z(t)
\end{array}\right]=\underbrace{\left[\begin{array}{c}
0 \\
F(t)
\end{array}\right]}_{B}, y(t)=\left[\begin{array}{ll}
0 & L
\end{array}\right]\left[\begin{array}{c}
\dot{z}(t) \\
z(t)
\end{array}\right]
$$




$$
\underbrace{\left[\begin{array}{cc}
0 & I \\
M & 0
\end{array}\right]}_{E}\left[\begin{array}{c}
\ddot{z}(t) \\
\dot{z}(t)
\end{array}\right]+\underbrace{\left[\begin{array}{cc}
-I & 0 \\
D & K
\end{array}\right]}_{A}\left[\begin{array}{c}
\dot{z}(t) \\
z(t)
\end{array}\right]=\underbrace{\left[\begin{array}{c}
0 \\
F(t)
\end{array}\right]}_{B}, y(t)=\left[\begin{array}{ll}
0 & L
\end{array}\right]\left[\begin{array}{c}
\dot{z}(t) \\
z(t)
\end{array}\right]
$$

The tests performed on all sixteen configurations were as follows:

1. The positive realness of the resulting transfer function, $H(s)$ was verified to show if $\operatorname{Re}\{H(s)\}=\operatorname{Re}\{s I-A\}=-\frac{1}{2}\left(A+A^{T}\right) \preceq-A \preceq 0$ to verify stability and passivity of the system,

2. The symmetry of the system matrices, $M, D$, and $K$ was verified as in Lemma 3.3,

3. The formulation of the first-order system was done by setting $W=-M$ and also by setting $W=-I$ in an attempt to see what impact this would have on the stability of the resulting reduced model.

\subsubsection{Results and Conclusions}

The results of testing the sixteen equivalent models of the second-order system conclusively showed that, of the sixteen available forms, only two passed the tests for stability and passivity. Even if the symmetry of the system was preserved by allowing $W=-M$, the tests still failed for the other fourteen forms.

Here, it was obvious that the formulation of the first-order system plays a very important role in achieving a stable and passive reduced model when implementing model reduction using 
the Arnoldi algorithm, and that the criteria for ensuring stability and passivity needs further consideration.

Many of the tests outlined above were supposed to have guaranteed at least the stability of the system, if not the passivity.

The two formulations that did work were

$$
\underbrace{\left[\begin{array}{cc}
M & D \\
0 & I
\end{array}\right]}_{E}\left[\begin{array}{c}
\ddot{z}(t) \\
\dot{z}(t)
\end{array}\right]+\underbrace{\left[\begin{array}{cc}
0 & K \\
-I & 0
\end{array}\right]}_{A}\left[\begin{array}{c}
\dot{z}(t) \\
z(t)
\end{array}\right]=\underbrace{\left[\begin{array}{c}
F(t) \\
0
\end{array}\right]}_{B}, y(t)=\left[\begin{array}{ll}
0 & L
\end{array}\right]\left[\begin{array}{c}
\dot{z}(t) \\
z(t)
\end{array}\right]
$$

$$
\underbrace{\left[\begin{array}{cc}
M & 0 \\
0 & I
\end{array}\right]}_{E}\left[\begin{array}{c}
\ddot{z}(t) \\
\dot{z}(t)
\end{array}\right]+\underbrace{\left[\begin{array}{cc}
D & K \\
-I & 0
\end{array}\right]}_{A}\left[\begin{array}{c}
\dot{z}(t) \\
z(t)
\end{array}\right]=\underbrace{\left[\begin{array}{c}
F(t) \\
0
\end{array}\right]}_{B}, y(t)=\left[\begin{array}{ll}
0 & L
\end{array}\right]\left[\begin{array}{c}
\dot{z}(t) \\
z(t)
\end{array}\right]
$$

In more recent work done in [59], additional criteria to ensure the passivity were introduced. These included ensuring the first moment of the system, $m_{0}$, was such that $m_{0} \geq 0$. In the implementation of the Arnoldi algorithm, the first solution used to build the orthogonal matrix is formed from the expansion around zero so the first moment is always zero, thus already satisfying the proposed criteria.

Also, model reduction must strive to achieve a stable and passive reduced order system if the original system was stable and passive. The system being modeled here is inherently stable and passive in the original second-order form. This infers that the SOAR procedure will always be stable and passive as the system retains its second-order form so the physical meaning of the 
system will never be lost.

The next three chapters of this thesis will compare the Arnoldi algorithm using the two configurations of the second-order system that passed the stability testing with the SOAR procedure.

A simple cantilever finite element model of three different mesh refinement will be considered, and the role of damping in building the reduced model will be carefully examined.

Furthermore, a comparison to the theoretical model of a cantilever will be included for the sake of completeness. 


\section{Chapter 5}

\section{Frequency Response of Krylov Based Model Reduction Techniques}

The frequency response of the three different sized cantilever models will be considered: a 540node and 2700-node cantilever will be examined here and a medium sized 1350-node model will be looked at in Appendix C. For the implementation of the Arnoldi algorithm, three different configurations will be used, including the two that met all the criteria for a stable response and a third that did not pass the stability tests but which is often used to demonstrate model reduction using the Arnoldi algorithm. The SOAR implementation remains as outlined in Chapter 3.

\subsection{Error Analysis}

The error presented in the frequency domain analysis of the cantilever is relative percentage error plotted on a $\log _{10}$ scale on the y-axis in order to illustrate the error levels more clearly. There is currently no real standard for acceptable error limits for model reductions, but the obvious choice is to have minimum error.

The relative percentage error, $\delta x$ is found as follows 


$$
\delta x=\frac{x_{0}-x}{x} \times 100
$$

Relative percentage error is also unitless.

\subsection{FEM Cantilever Model}

\subsubsection{Cantilever Models with Damping $=0 \times M$}

As with the comparisons performed in the time domain, the cantilever is modeled with different model sizes as well as different levels of damping. Here, the damping was set to $D=0 \times M$, and the frequency response for all three cantilever models was evaluated.

\section{0-node Cantilever}

The frequency response for this undamped cantilever is given in Figure 5.1 


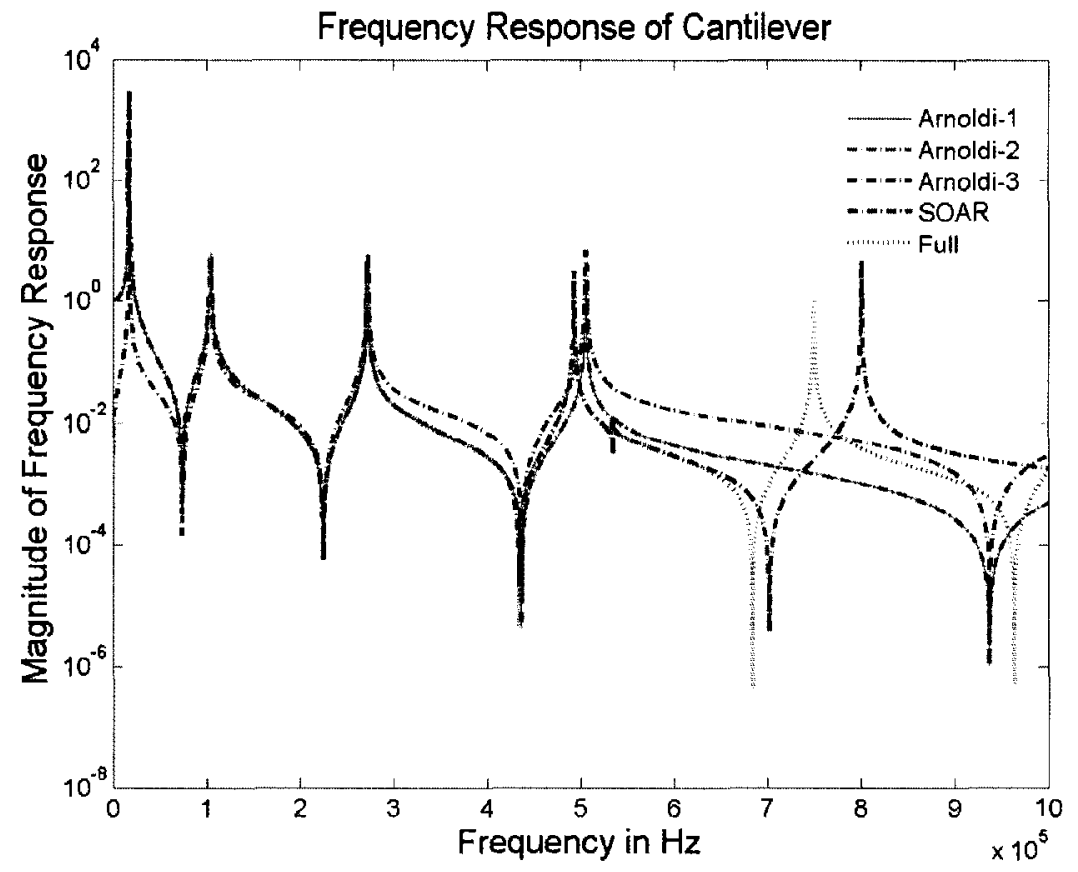

(a) Arnoldi-1, Arnoldi-2, Arnoldi-3, SOAR

Figure 5.1: Comparison of frequency response for the full system and reduced model of a 540 node Cantilever with Damping $=0 \times M$ 


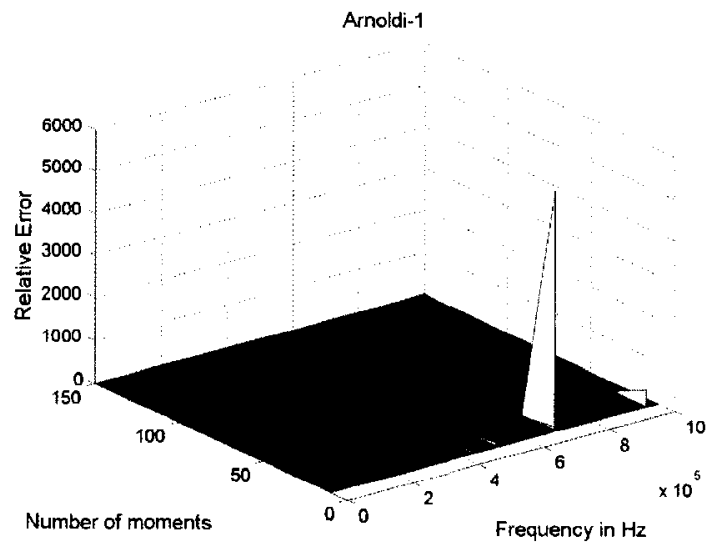

(a) Arnoldi-1

Arnoldi-3

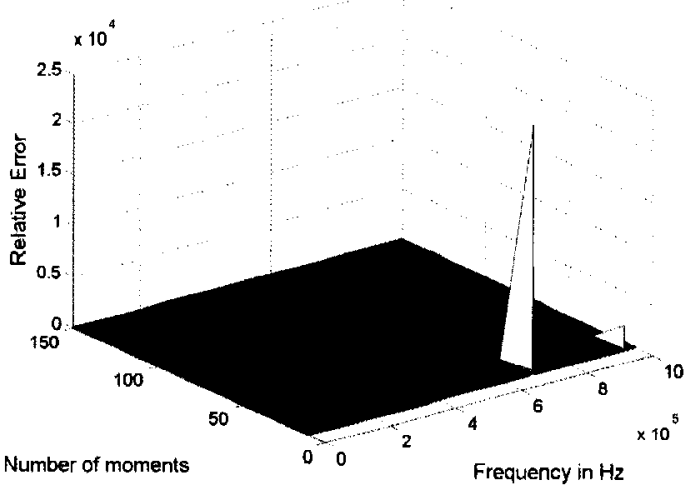

(c) Arnoldi-3

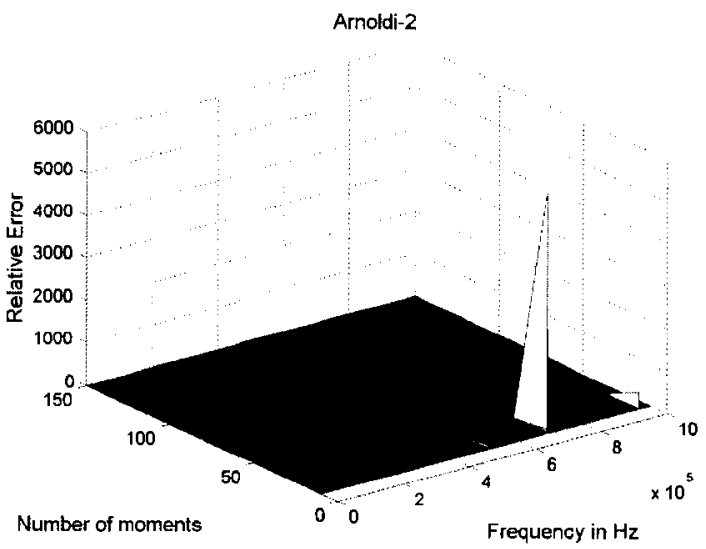

(b) Arnoldi-1

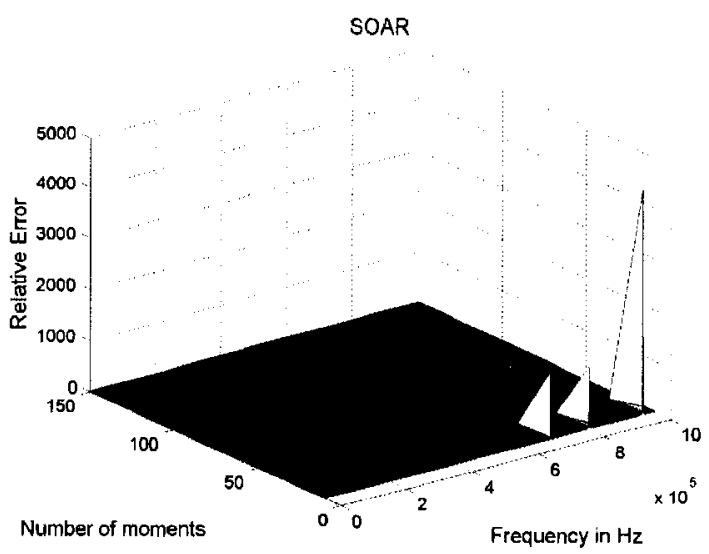

(d) SOAR

Figure 5.2: Relative Error for the Frequency Response for 540-node cantilever, Damping=0 $\times M$ 
Discussion The frequency response for the 540-node can be shown in Figure 5.1. It was observed that the relative percentage error in the frequency response was small at frequencies lower than $<4 \times 10^{5} \mathrm{~Hz}$ for a low number of moments, all three companion forms, and for both the Arnoldi algorithm and SOAR, on the order of $<<1 \%$. At higher frequencies, the relative percentage error increased dramatically and was clearly seen by the spike in Figure 5.2. However, if the number of moments taken was $\geq 30$ the error stayed within acceptably low levels. It was also noted that the magnitude of the relative error was approximately the same for all three companion forms of the Arnoldi algorithm.

\section{0-node Cantilever}

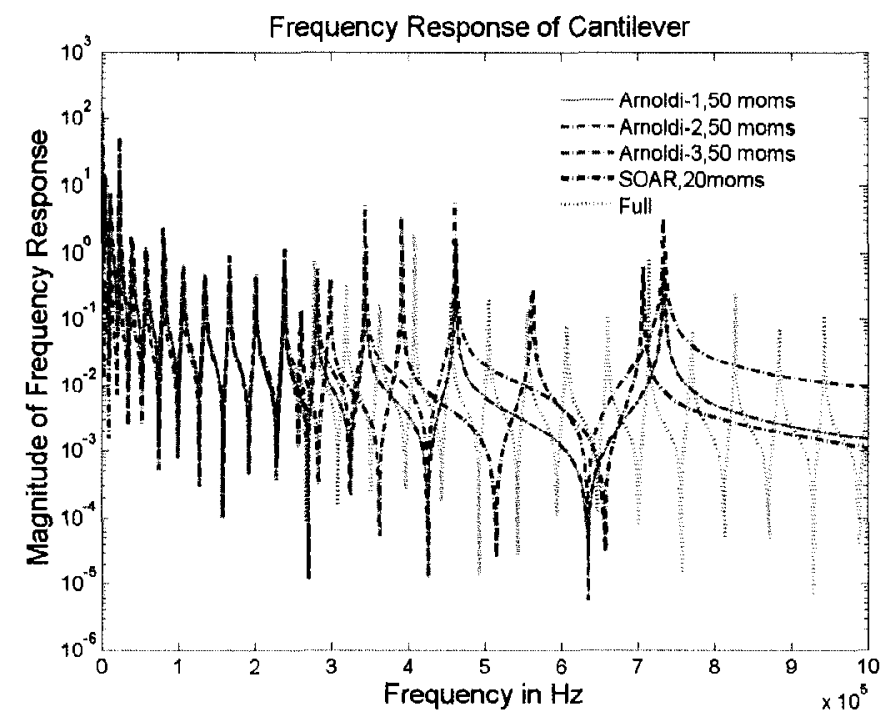

(a) Arnoldi-1, Arnoldi-2, Arnoldi-3, SOAR

Figure 5.3: Comparison of frequency response for the full system and reduced model of a 2700 node Cantilever with Damping $=0 \times M$ 


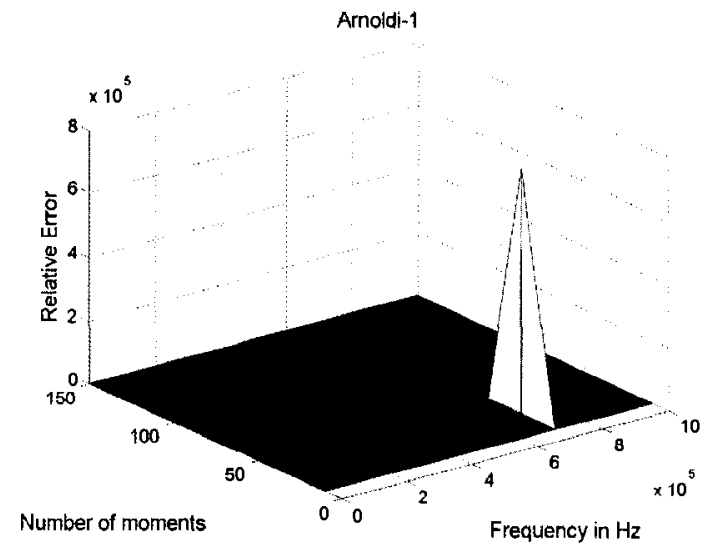

(a) Arnoldi-1

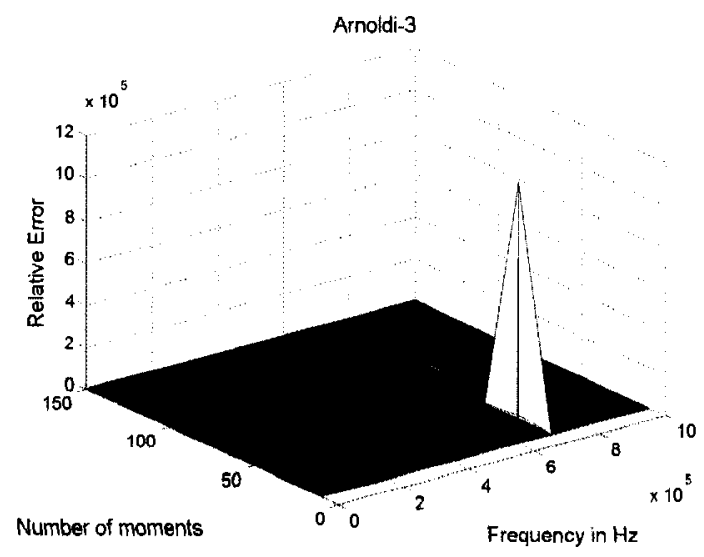

(c) Arnoldi-3

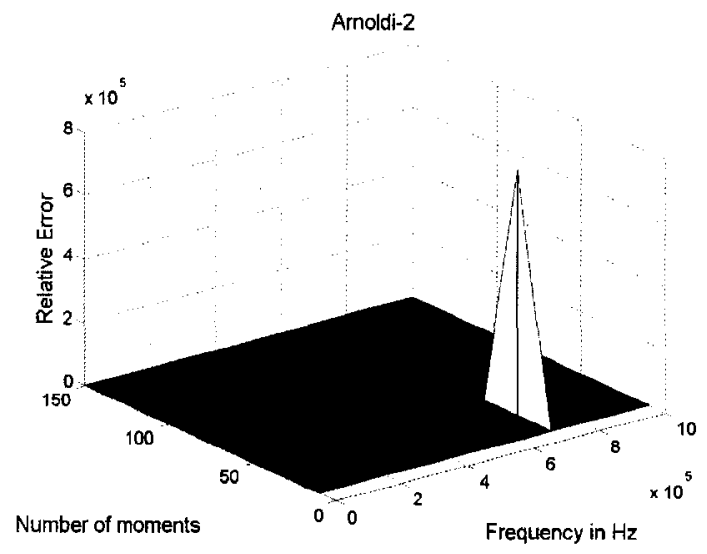

(b) Arnoldi-2

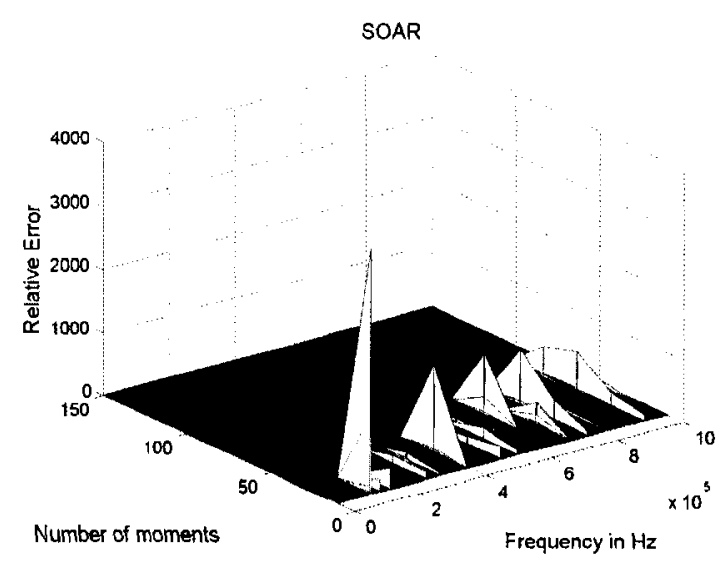

(d) SOAR

Figure 5.4: Relative Percentage Error for the Frequency Response for 2700-node cantilever, Damping $=0 \times M$ 
Discussion There seemed to be a greater observable relative percentage error in the frequency response for the 2700-node cantilever as shown in Figure 5.3, and a larger number of moments was needed for both SOAR and Arnoldi to give a good match at lower frequencies. This was expected due to the larger size of the model. It was observed that both model reduction techniques performed well at lower frequencies but at frequencies greater than $4 \times 10^{5} \mathrm{~Hz}$, the amount of error was much higher. It was also interesting to note that all three companion forms performed comparably but, as shown in Figure 5.4, the relative percentage errors for the SOAR procedure demonstrated a greater amount of error at all frequencies for a lower number of moments. 
5.2.2 Cantilever Models with Damping $=10^{-2} \times M$

540-node Cantilever

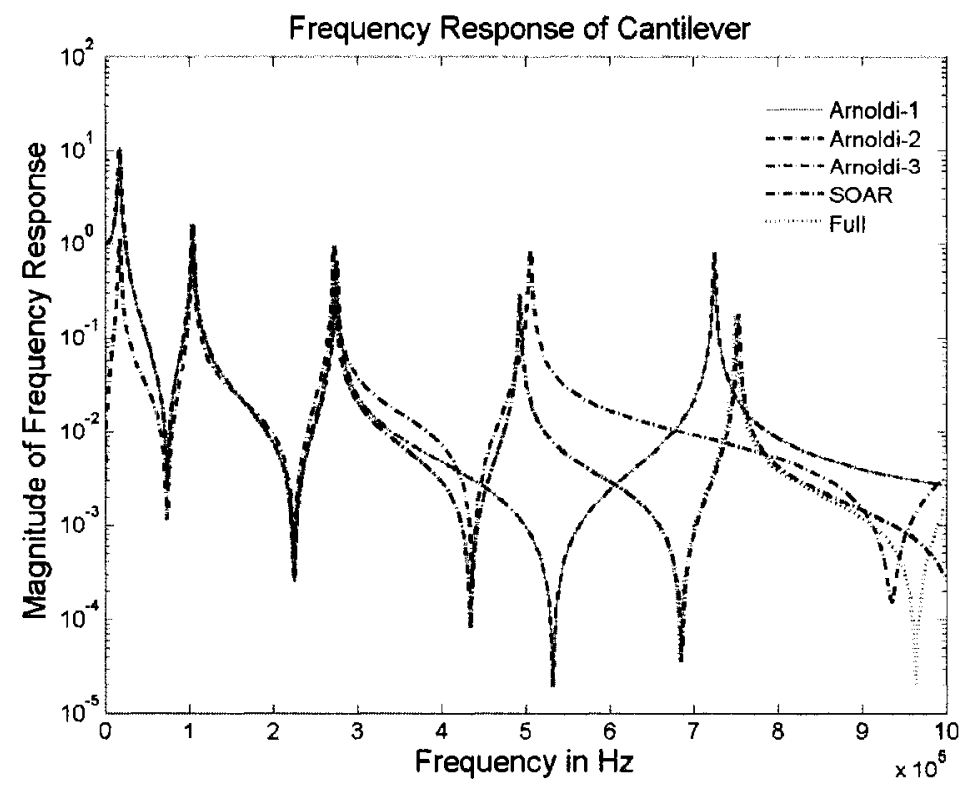

(a) Arnoldi-1, Arnoldi-2, Arnoldi-3, SOAR

Figure 5.5: Comparison of frequency response for the full system and reduced model of a 540 node Cantilever with Damping $=10^{-2} \times M$ 


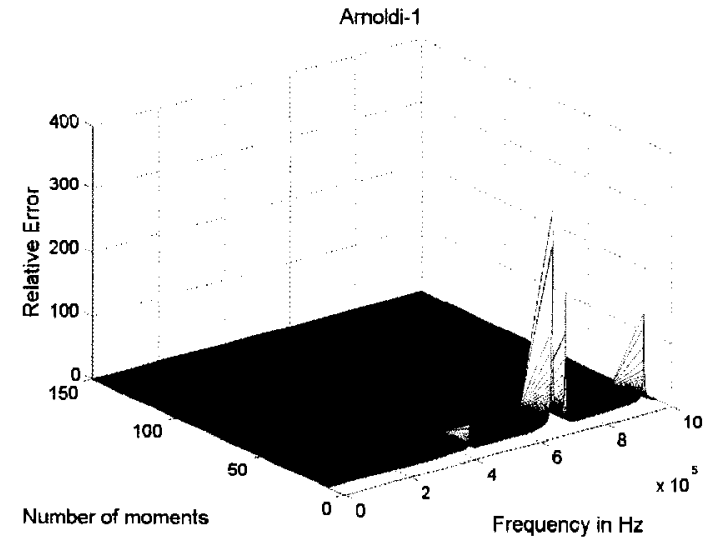

(a) Arnoldi-1

Arnoldi-3

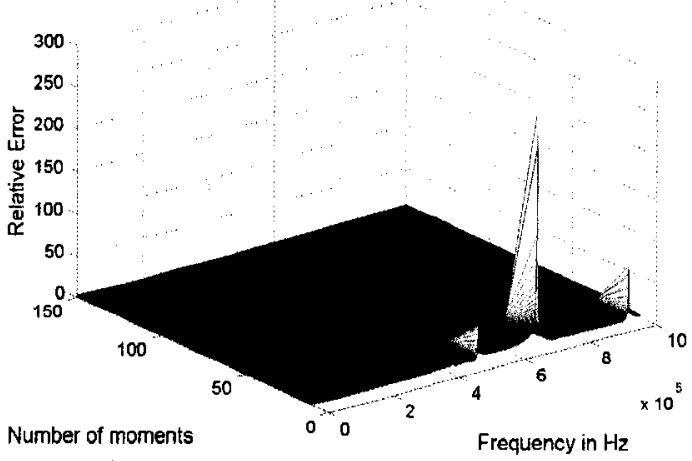

(c) Arnoldi-3

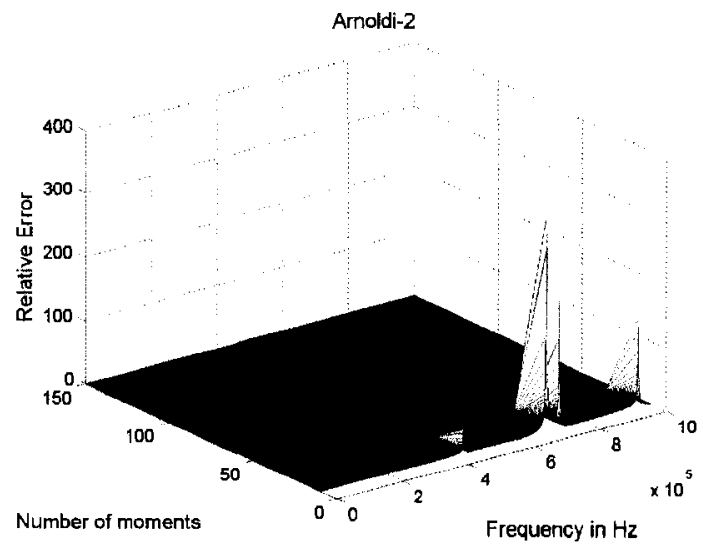

(b) Arnoldi-2 SOAR

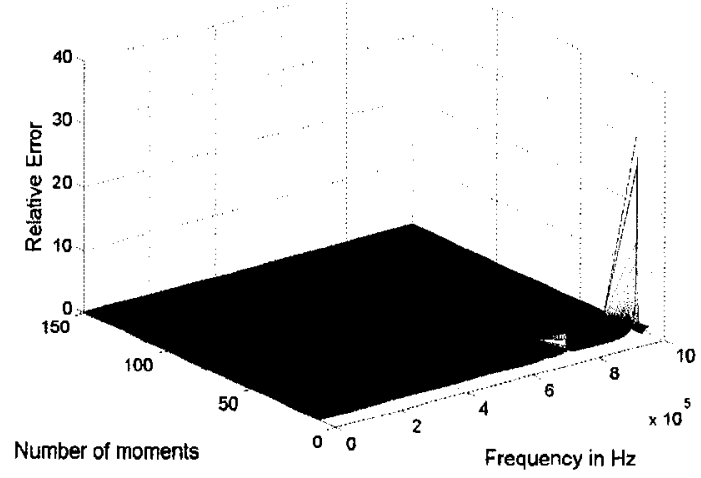

(d) SOAR

Figure 5.6: Relative Percentage Error for the Frequency Response for 540-node cantilever, Damping $=10^{-2} \times M$ 
Discussion The frequency response shown in Figure 5.5 indicated that the configuration used in forming the companion form used in the Arnoldi algorithm is an important factor in the frequency response domain analysis since the level of error shown for this model in Figure 5.6 was still comparable for all three companion forms. For SOAR, as the number of moments increased the range of frequency that can be matched to the full response also increased.

\section{0-node Cantilever}

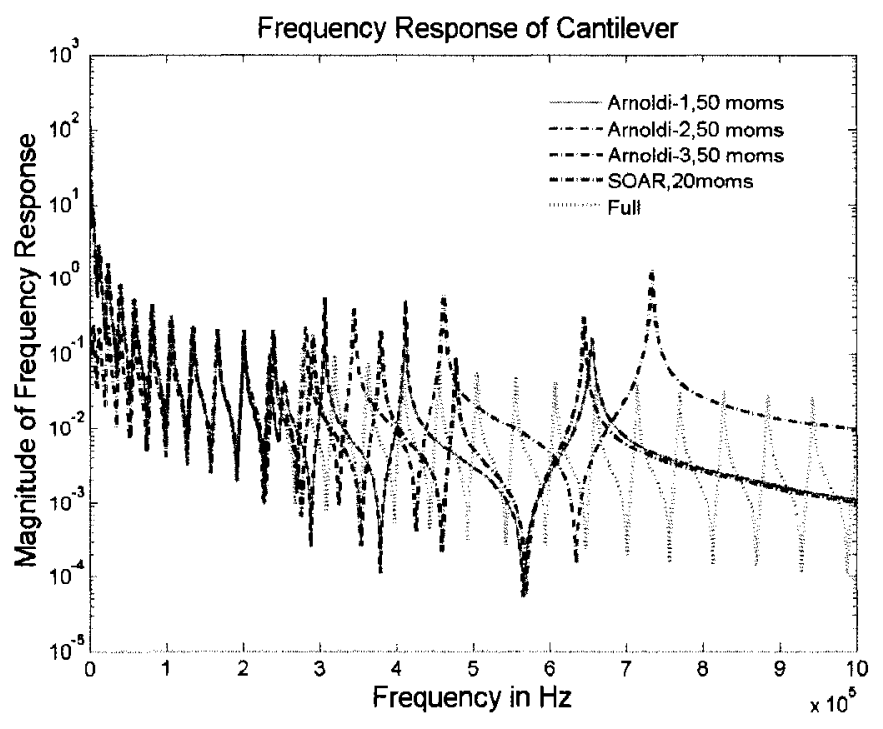

(a) Arnoldi-1, Arnoldi-2, Arnoldi-3, SOAR

Figure 5.7: Comparison of frequency responses for the full system and reduced model of a 2700-node Cantilever with Damping $=10^{-2} \times M$ 


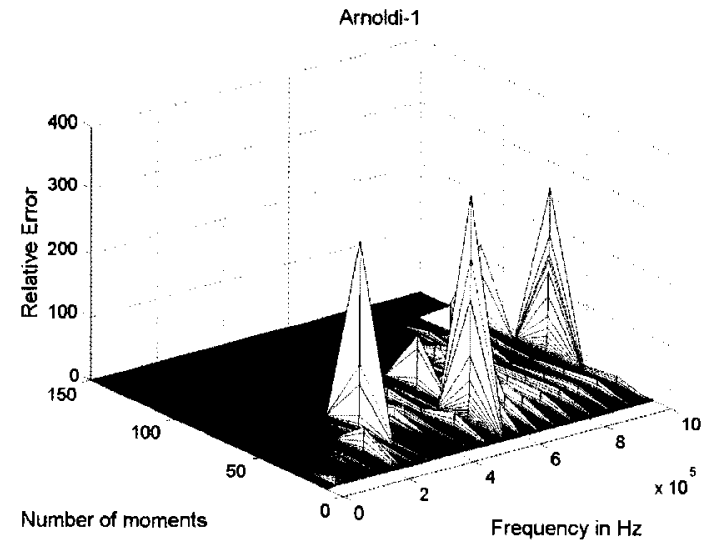

(a) Arnoldi-1

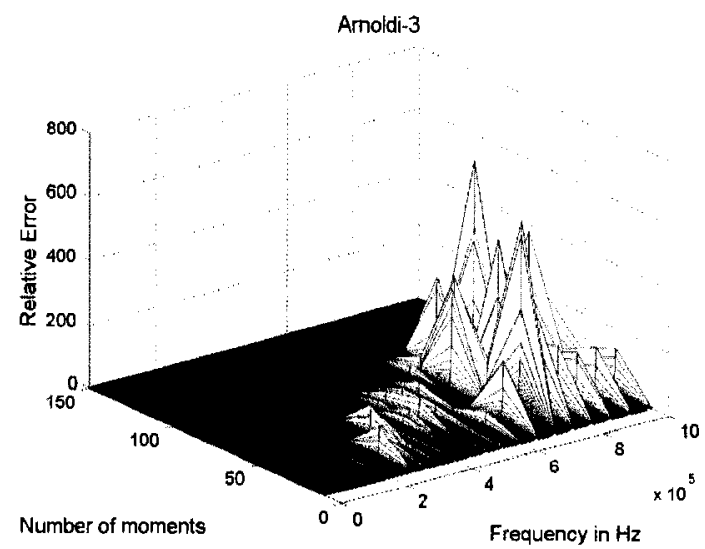

(c) Arnoldi-3

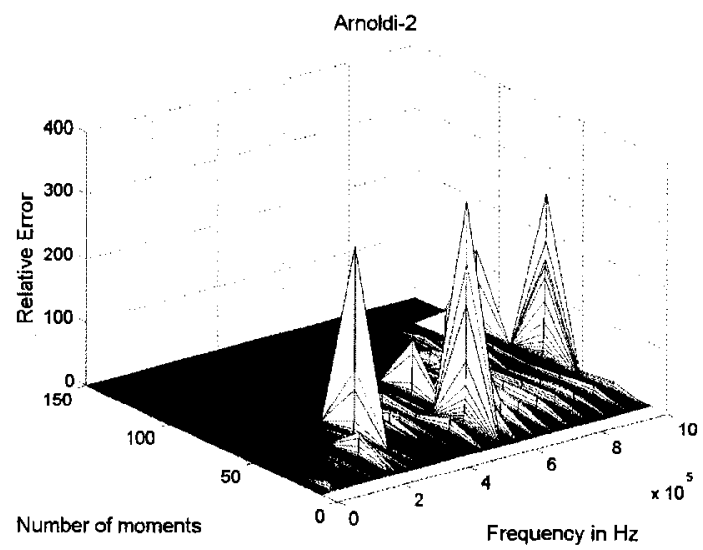

(b) Arnoldi-2

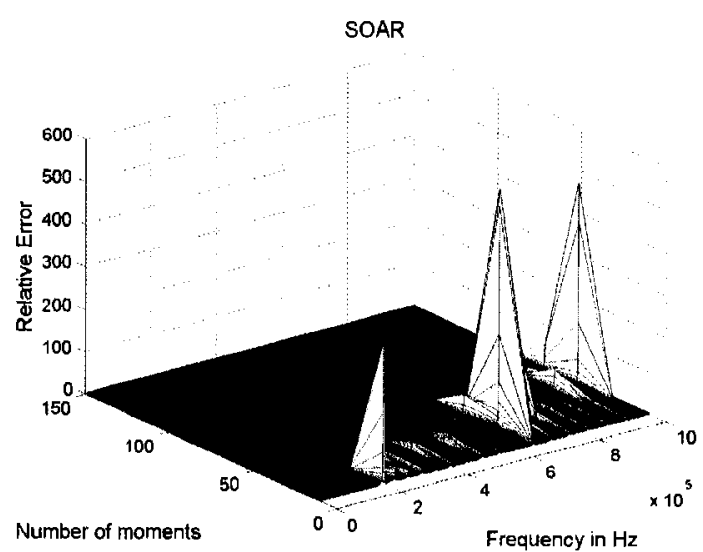

(d) SOAR

Figure 5.8: Relative Percentage Error for the Frequency Response for 2700-node cantilever, Damping $=10^{-2} \times M$ 
Discussion The results shown for the 2700-node cantilever at $D=10^{-2} \times M$ indicated that there was a bigger discrepancy in the frequency response of the reduced model and the full models at lower frequencies, see Figure 5.7. In addition, Figure 5.8 showed that at higher levels of damping, differences in the relative percentage error between the different companion forms used in the Arnoldi algorithm were more evident. However, all three forms and SOAR demonstrated much higher levels of error at higher frequencies. This indicated that SOAR presented an advantage by minimizing the relative percentage error at higher frequencies if enough moments were taken, while the Arnoldi algorithm did not.

\subsubsection{Cantilever Models with Damping $=10^{-1} \times M$}

\section{0-node Cantilever}

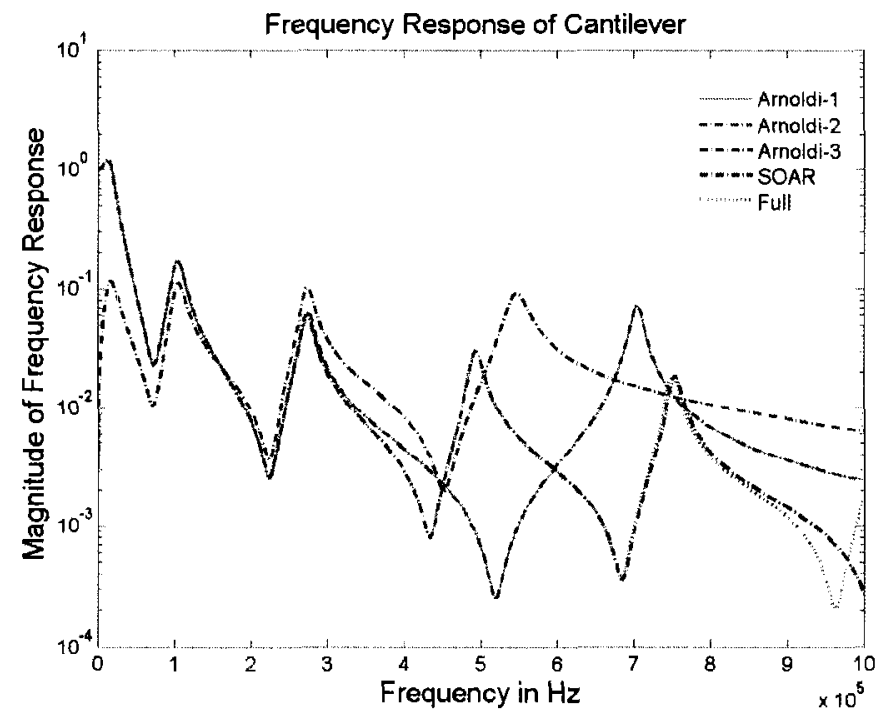

(a) Arnoldi-1, Arnoldi-2, Arnoldi-3, SOAR

Figure 5.9: Comparison of frequency response for the full system and reduced model of a 540 node Cantilever with Damping $=10^{-1} \times M$ 


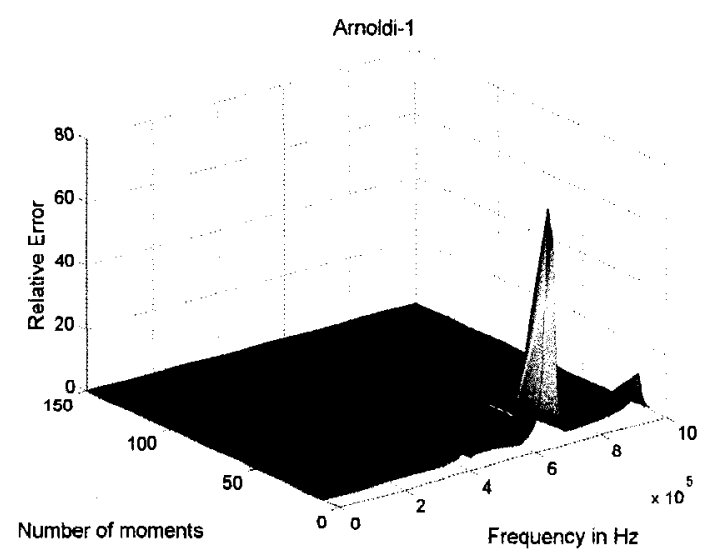

(a) Arnoldi-1

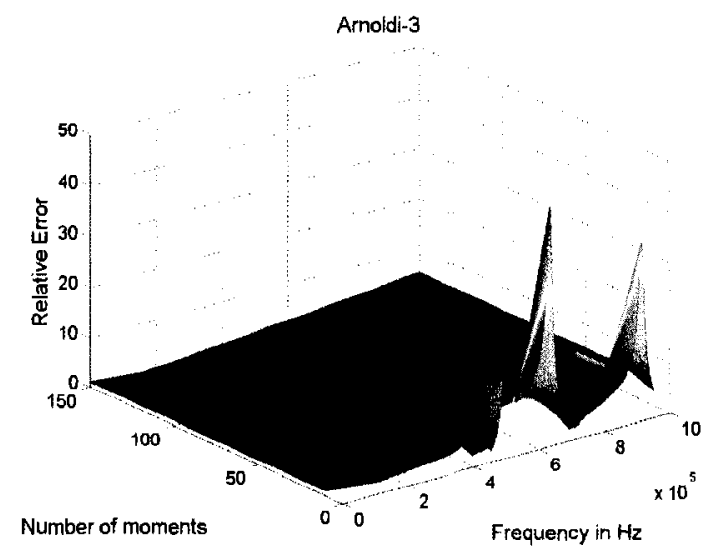

(c) Arnoldi-3

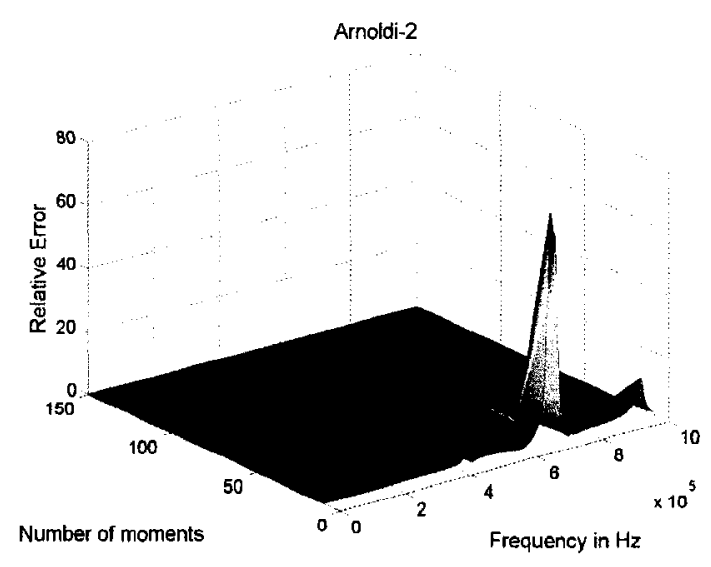

(b) Arnoldi-2

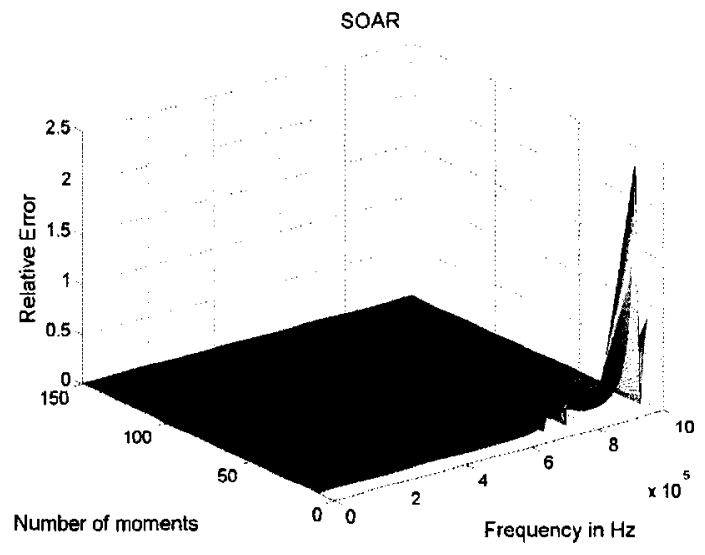

(d) SOAR

Figure 5.10: Relative Percentage Error for the Frequency Response for 540-node cantilever, Damping $=10^{-1} \times M$ 
Discussion For a damping $D=0.1 \times M$, the third representation of the second-order system showed a much higher level of error while all the other stable forms and SOAR provided a good match for the 540-node FEM cantilever, see Figure 5.9. SOAR, however, had lower relative errors at a higher frequency cut-off point with only 20 moments. At a higher number of moments, the relative error decreased significantly as shown in Figure 5.10.

\section{0-node Cantilever}

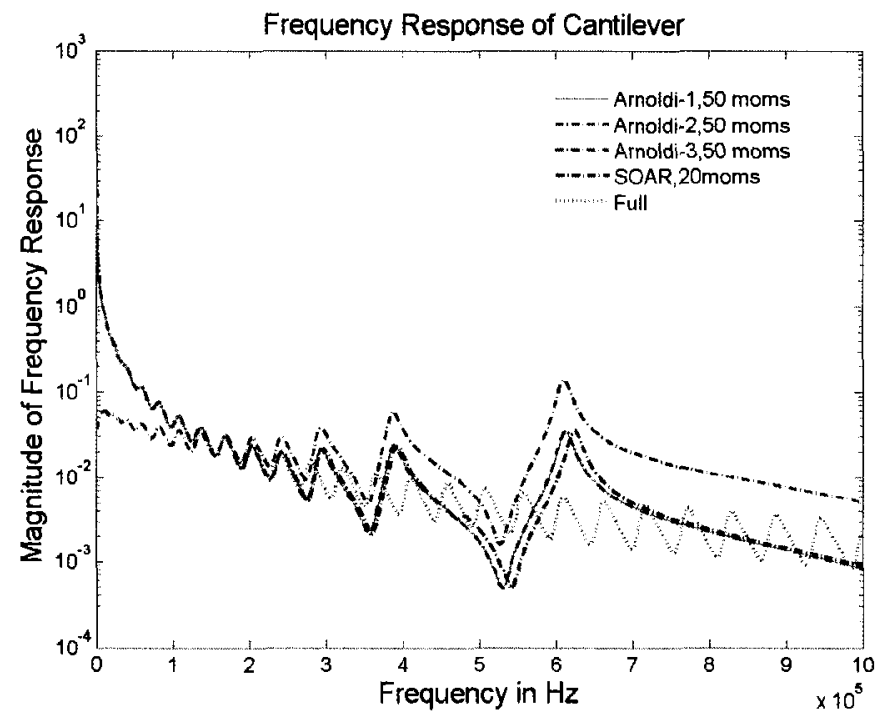

(a) Arnoldi-1, Arnoldi-2, Arnoldi-3, SOAR

Figure 5.11: Comparison of frequency response for the full system and reduced model of a 2700 node Cantilever with Damping $=10^{-1} \times M$ 


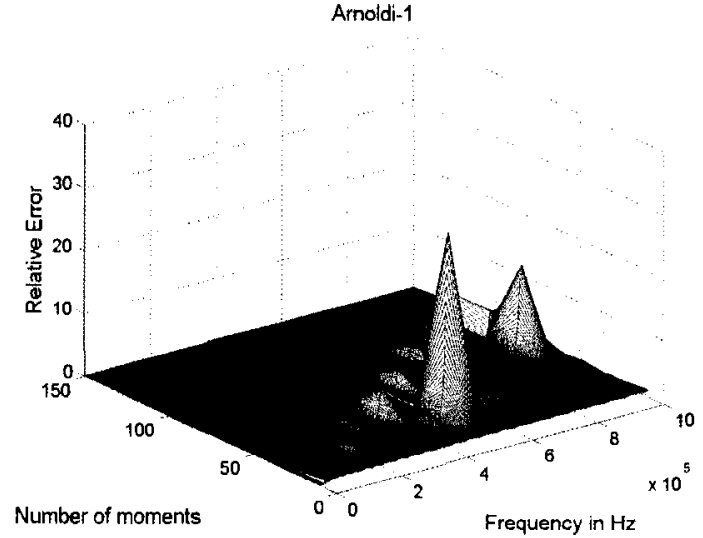

(a) Arnoldi-1

Arnoldi-3

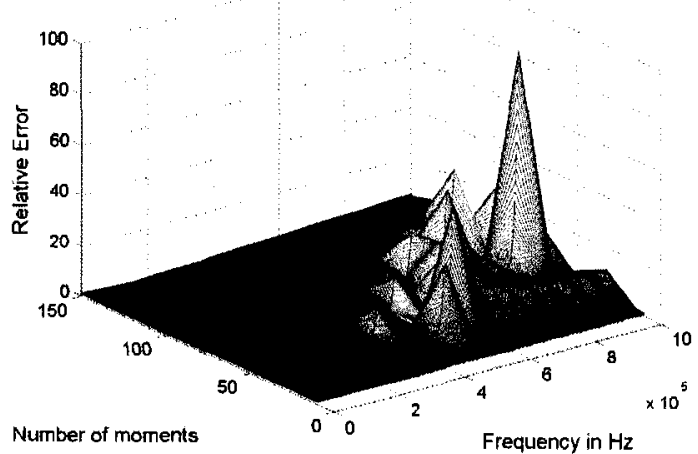

(c) Arnoldi-3

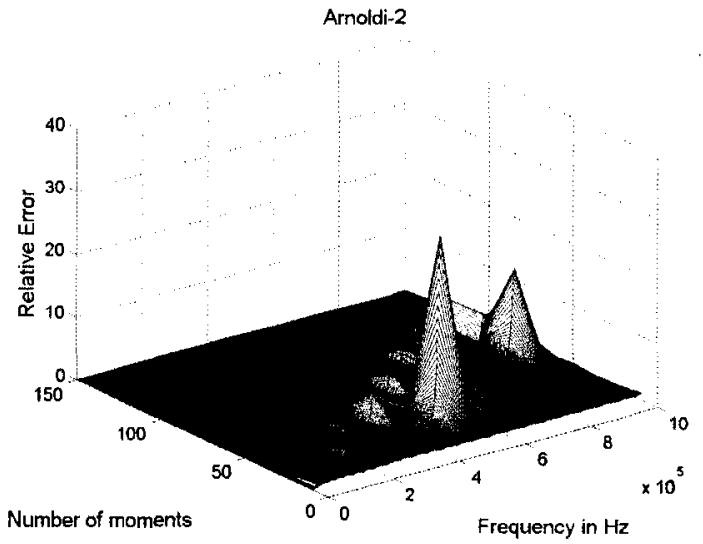

(b) Arnoldi-2 SOAR

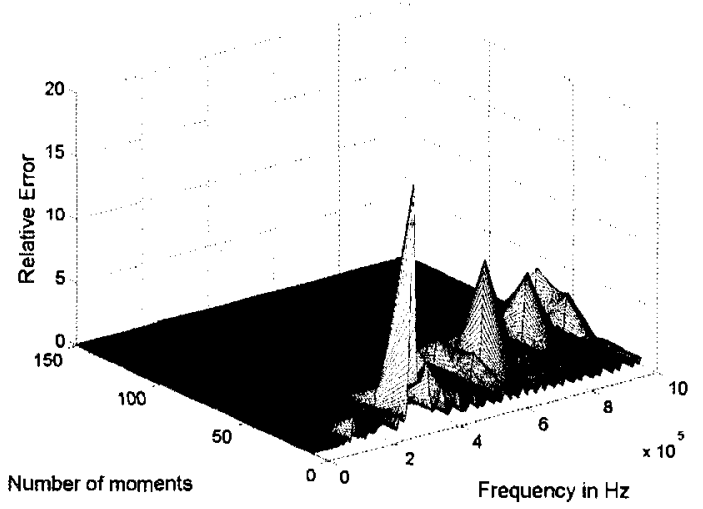

(d) SOAR

Figure 5.12: Relative Percentage Error for the Frequency Response for 2700-node cantilever, Damping $=10^{-1} \times M$ 
Discussion The frequency response for the 2700-node cantilever clearly demonstrated that the third companion form used for the implementation of the Arnoldi algorithm resulted in much greater inaccuracy in its response compared to the full system, as seen in Figure 5.12, but the response for all the model reduction techniques at 50 moments for Arnoldi and 20 for SOAR, in Figure 5.10, was quite poor. SOAR exhibited a better response at higher frequencies. However, it was also seen that, with a higher number of moments, a more accurate frequency response can be found for a wider range of frequencies for all the algorithms.

\subsubsection{Conclusion on Frequency Response of FEM Models}

The frequency domain analysis of the FEM models presented in this section showed that all three companion forms of the second-order system that passed stability tests and were used to carry out model reduction with the Arnoldi algorithm performed approximately the same. However, at higher damping, the third form began to deviate from the other two companion forms in its level of error and response to the full model. Also, the range of frequency in which the frequency response matched the full response was smaller, indicating that any form of the companion form could be used if the operating frequency range required was within a specific range.

Beyond that, there did not seem to be any significant difference between SOAR and Arnoldi as they both had good relative percentage error thresholds, and displayed a good match to the full model. It was interesting and important to note that the companion form used for the implementation of Arnoldi did not seem to be relevant for the frequency domain analysis 
especially for lower frequency ranges and if a sufficient number of moments was taken.

\subsection{SUGAR Models}

The frequency response for two SUGAR models will now be considered. The first model is comprised of 150 elements and has 450 nodes, and the second has 500 elements and 1500 nodes.

The response achieved by varying damping for moments ranging from 10 to 100 will be shown.

\subsubsection{SUGAR Models and the Arnoldi Algorithm}

The SUGAR models created here were preconditioned by scaling the matrices to compensate for ill-conditioning problems all models created with SUGAR displayed. Since preconditioning was absolutely necessary for the time domain analysis, it was felt that the same scaling would be implemented in the frequency domain in order to ensure that the models being analyzed were numerically similar in every way possible.

Figures 5.14 and 5.16 show the relative percentage error achieved for these models using SOAR.

\section{0-element SUGAR model}

The frequency response of a 150-element SUGAR model is presented below. The damping was increased from $10^{15}$ to $10^{19}$. The value for which the damping was set in SUGAR is different than FEM, as it was simply easier to scale the SUGAR damping matrix than it was to find the 
equivalent scaling factor for the mass matrix. This still resulted in a scaled mass matrix, but this method achieved a stable response with less tweaking of the scaling factor.

Figure 5.13 shows the frequency response of the reduced SUGAR model compared to the full model of the 150-element and Figure 5.14 examines the relative percentage error of the reduced model.
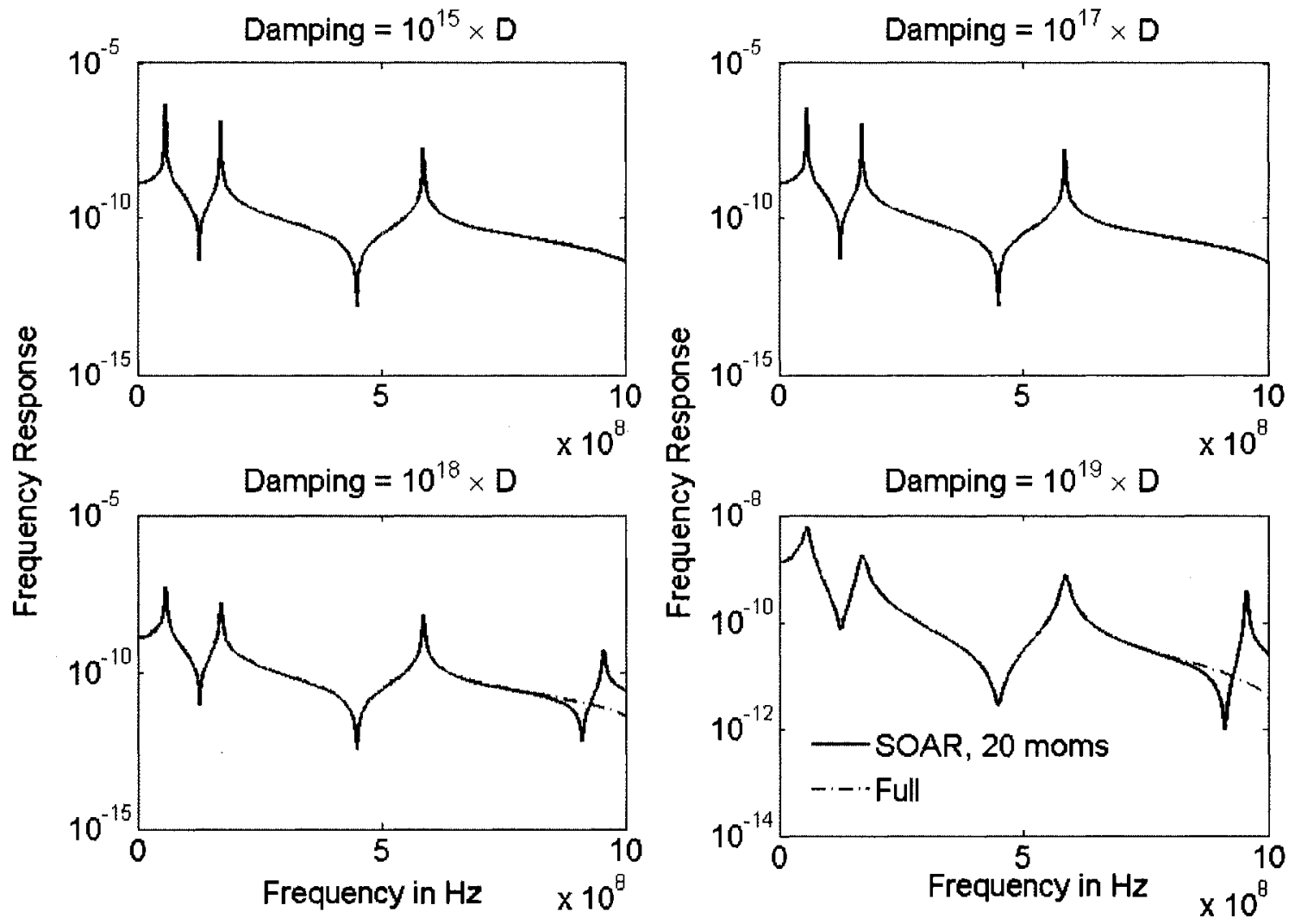

Figure 5.13: Frequency Response for a SUGAR Model with 150 elements using Arnoldi

\section{0-element SUGAR model}

A much larger 500-element SUGAR model is now examined. Recall, SUGAR was designed to model a very large number of elements simply, so the accuracy of model reduction is crucial to 

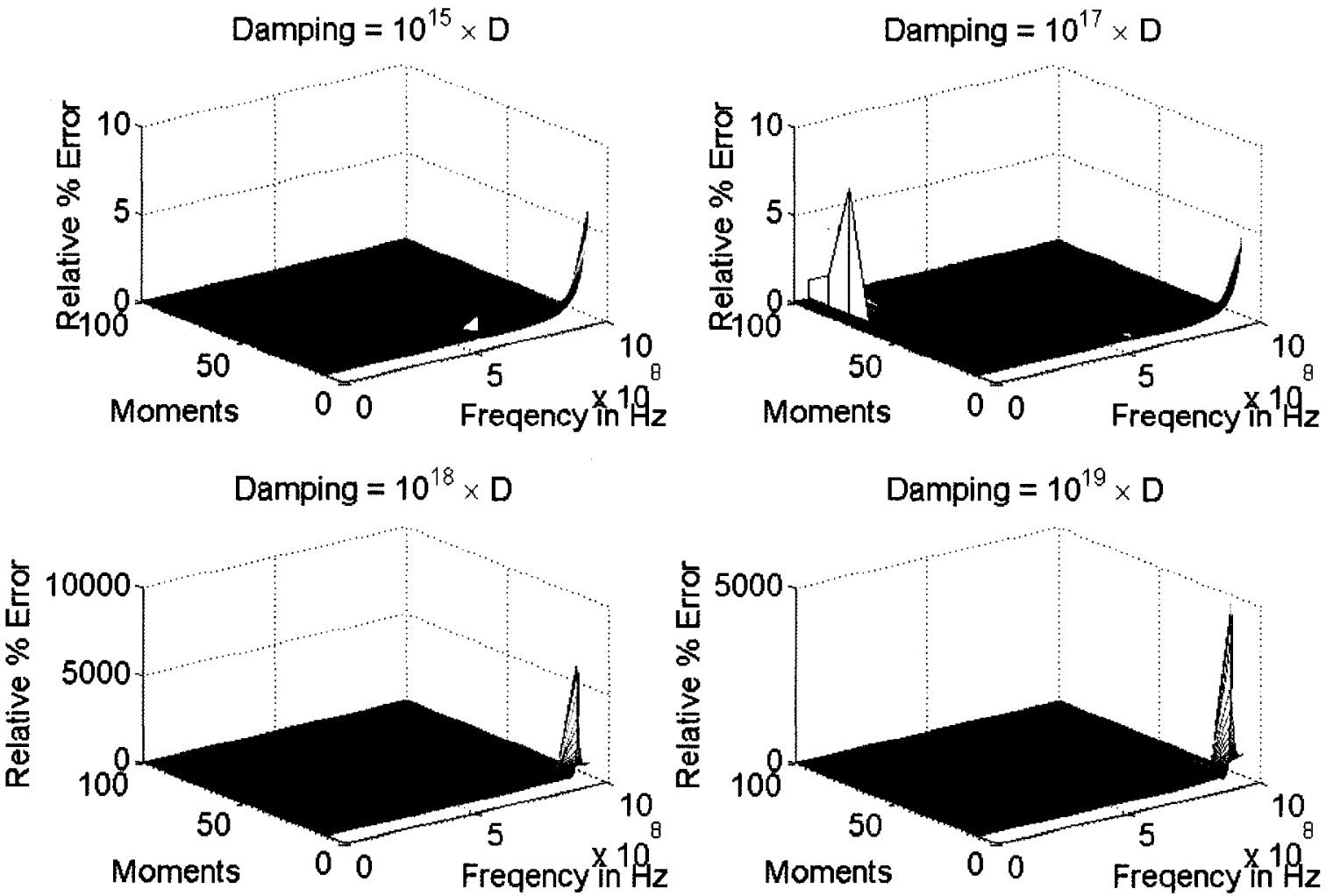

Figure 5.14: Relative Percentage Error of the Frequency Domain Response for a SUGAR Model with 150 elements using Arnoldi

the overall response of the system.

Figure 5.15 represents the frequency response of the reduced SUGAR 500-element model compared to the full model and Figure 5.16 examines the relative percentage error of the reduced model. 

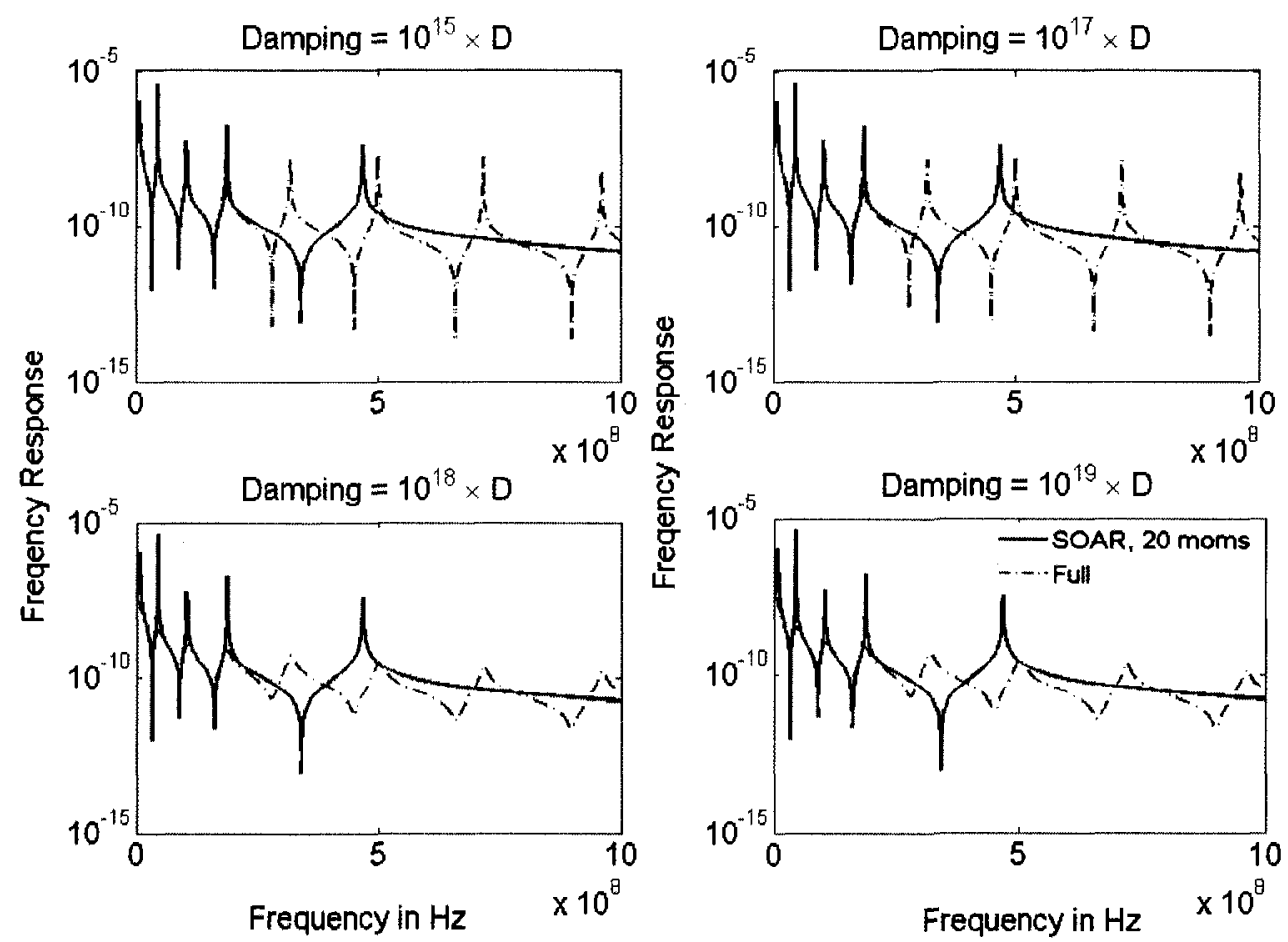

Figure 5.15: Frequency Response for a SUGAR Model with 500 elements using Arnoldi 

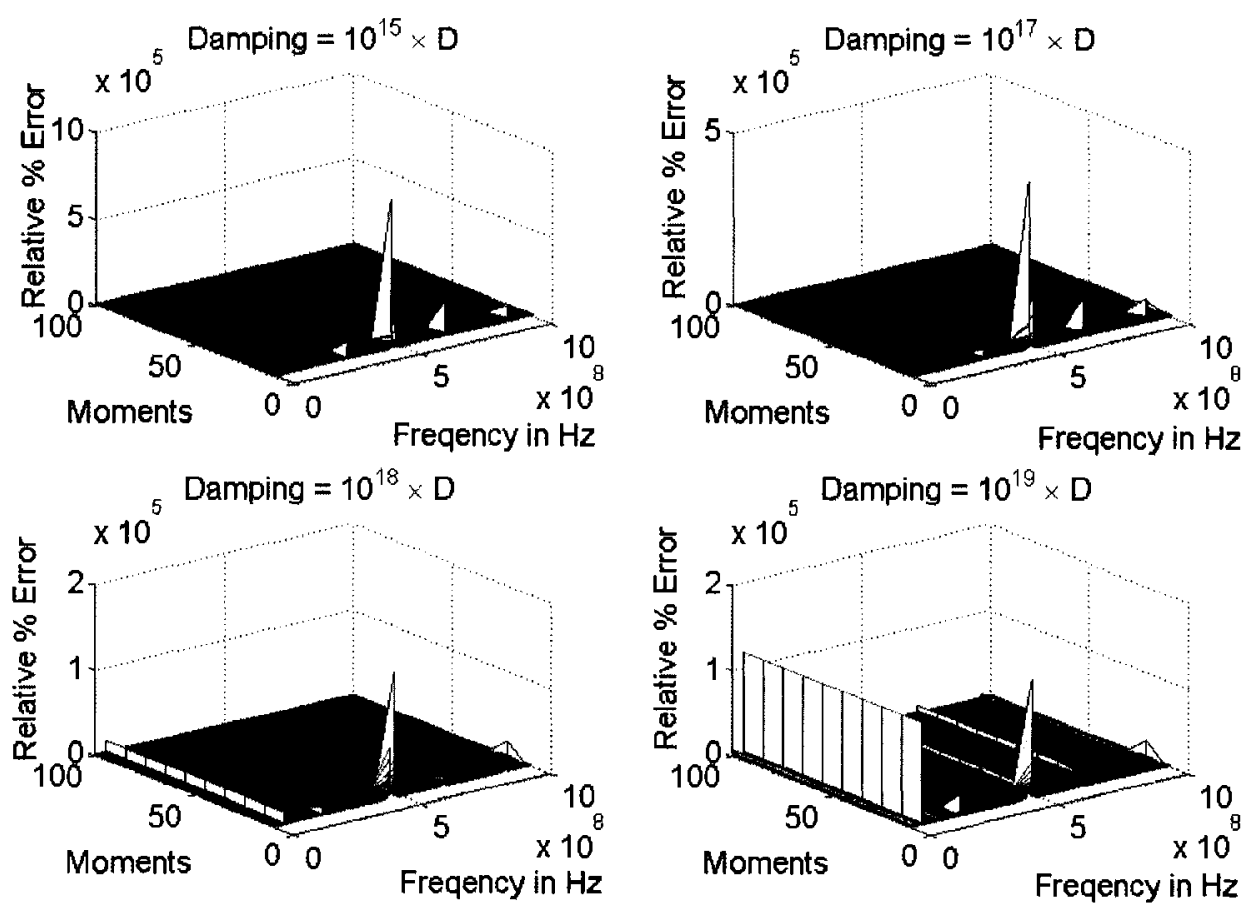

Figure 5.16: Relative Percentage Error of the Frequency Domain Response for a SUGAR Model with 500 elements using Arnoldi

Discussion From the above analysis, the Arnoldi algorithm provided a good match to the full frequency response of models generated using SUGAR. At higher frequencies and higher amounts of damping, the relative percentage error was quite large, but this served as a measure for the range of frequency in which the model reduction has optimal performance. The results also showed that not many moments were required to achieve a favorable response and increasing the number of moments at higher frequencies also increased the frequency range for which the Arnoldi model reduction provided a good match to the full model. 


\subsubsection{SUGAR Models and the SOAR Procedure}

Although the matrices are similarly preconditioned for the implementation of SOAR, as they were for the Arnoldi algorithm, any attempt at scaling only resulted in scaling the original system matrices. As a result, the condition number did not change as it did for the companion form system used in the Arnoldi algorithm. This implies that Arnoldi has an advantage over SOAR for models generated using SUGAR.

\section{0-element SUGAR model}

Figure 5.17 shows the frequency response for the 150 element SUGAR model reduced using SOAR. As with the Arnoldi algorithm, the damping is increased from $10^{15}$ to $10^{19}$. 

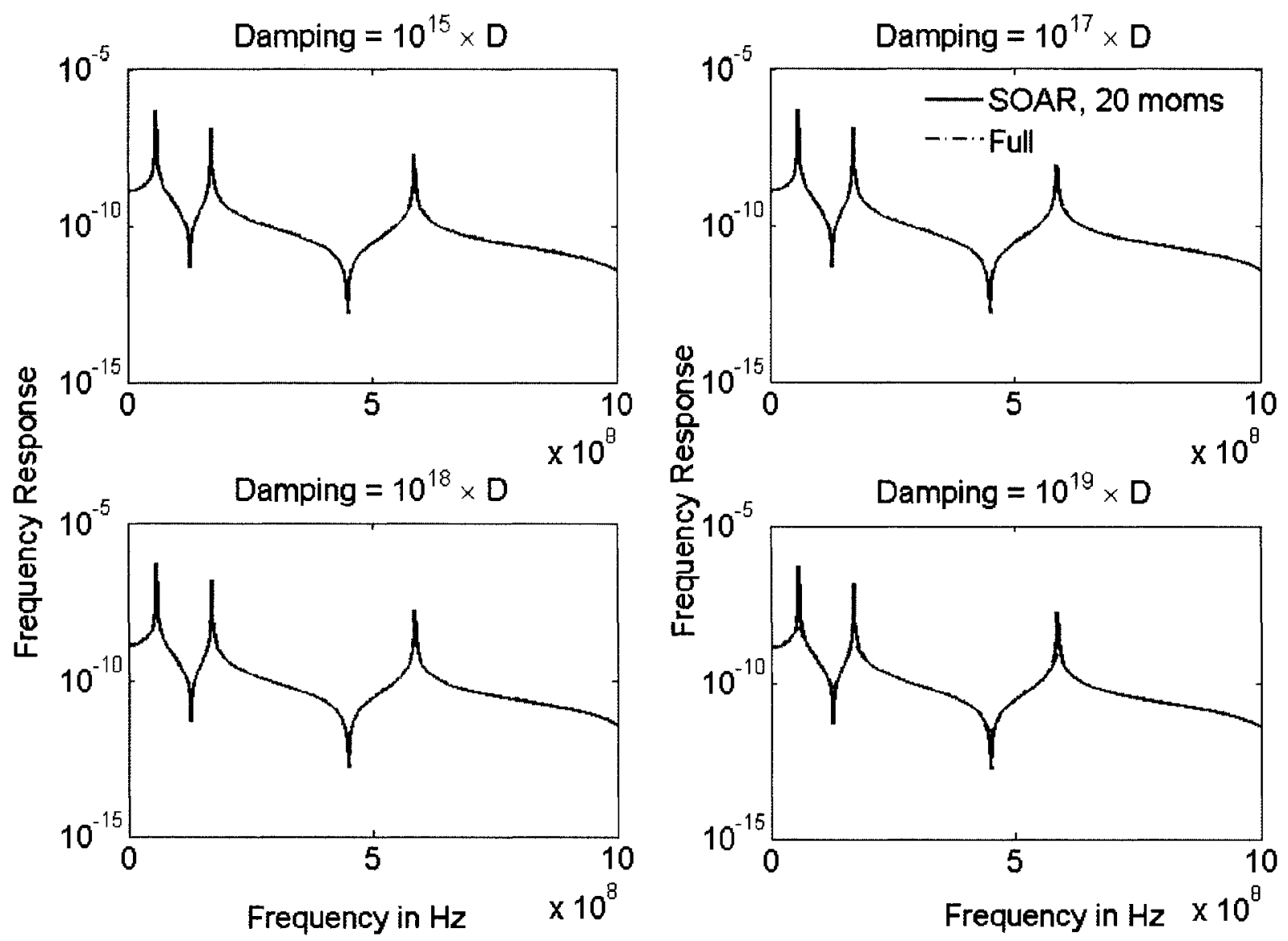

Figure 5.17: Frequency Response for a SUGAR Model with 150 elements using SOAR 

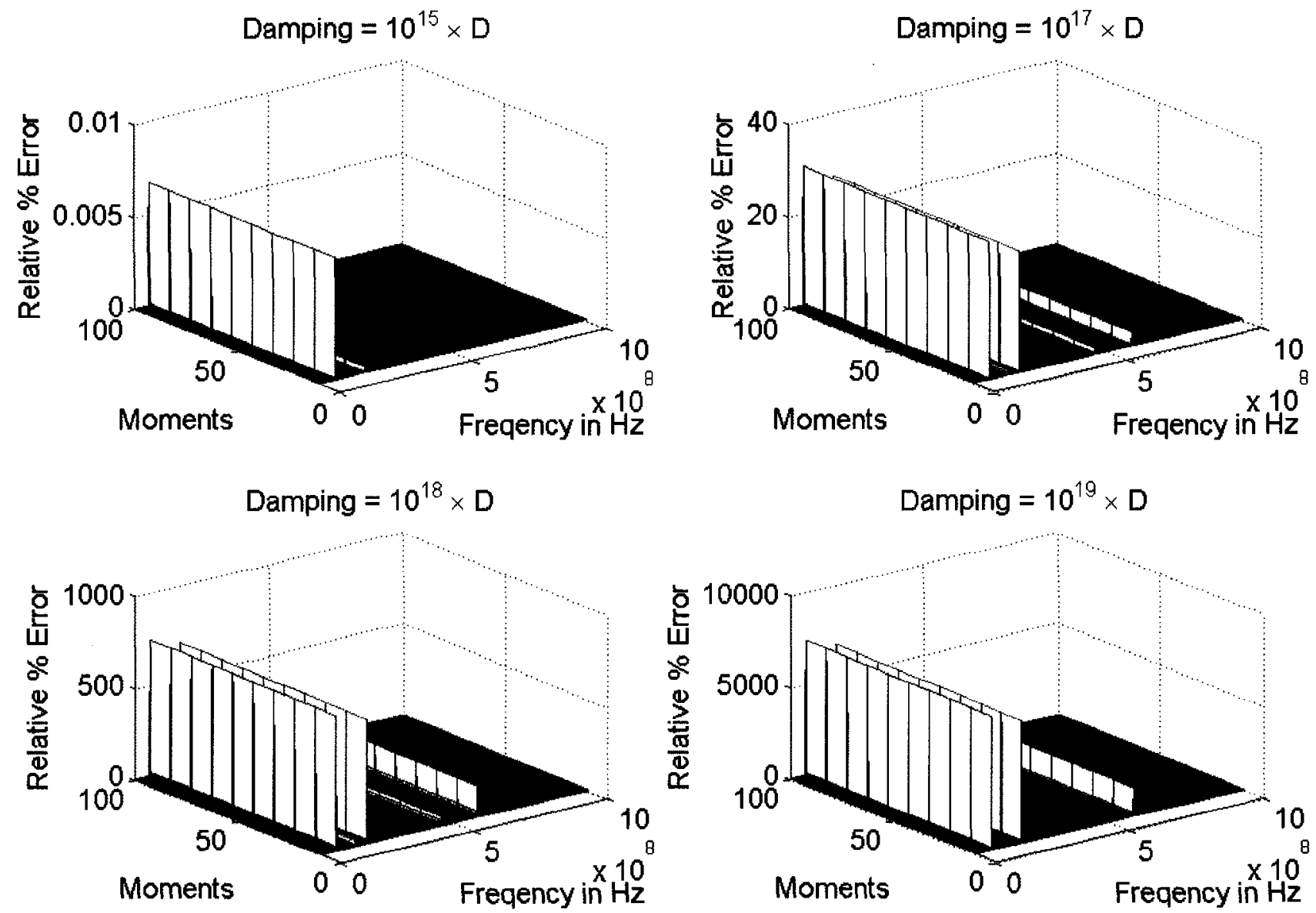

Figure 5.18: Relative Percentage Error of the Frequency Domain Response for a SUGAR Model with 150 elements using SOAR 


\section{0-element SUGAR Model}

The model reduction of the larger 500-element SUGAR model will now be examined with the implementation of the SOAR procedure as the model reduction technique of choice.

Figure 5.19 shows the frequency response of the reduced system and full system respectively and Figure 5.20 illustrates the relative percentage error between the full and reduced models.
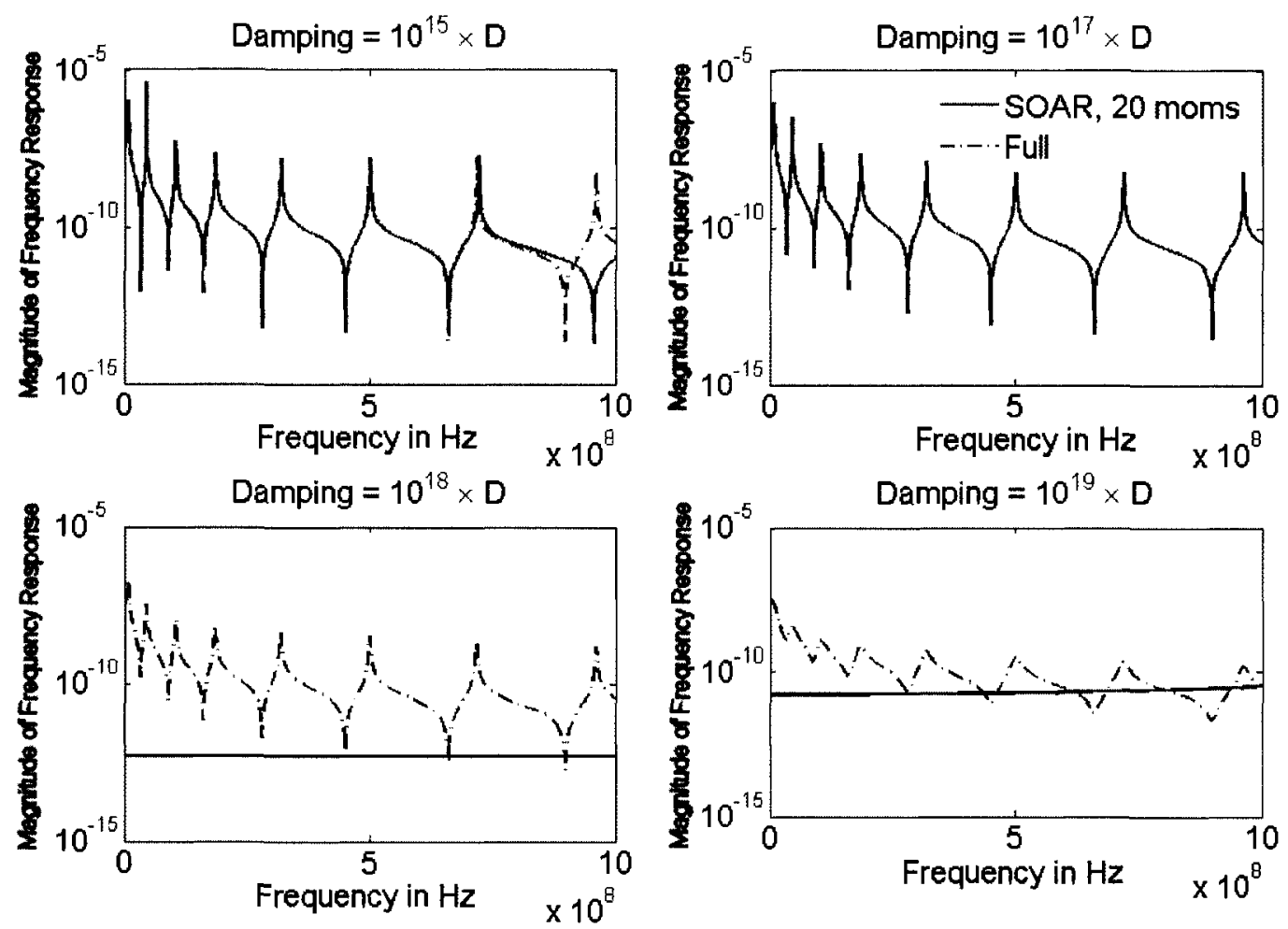

Figure 5.19: Frequency Response for a SUGAR Model with 500 elements using SOAR 

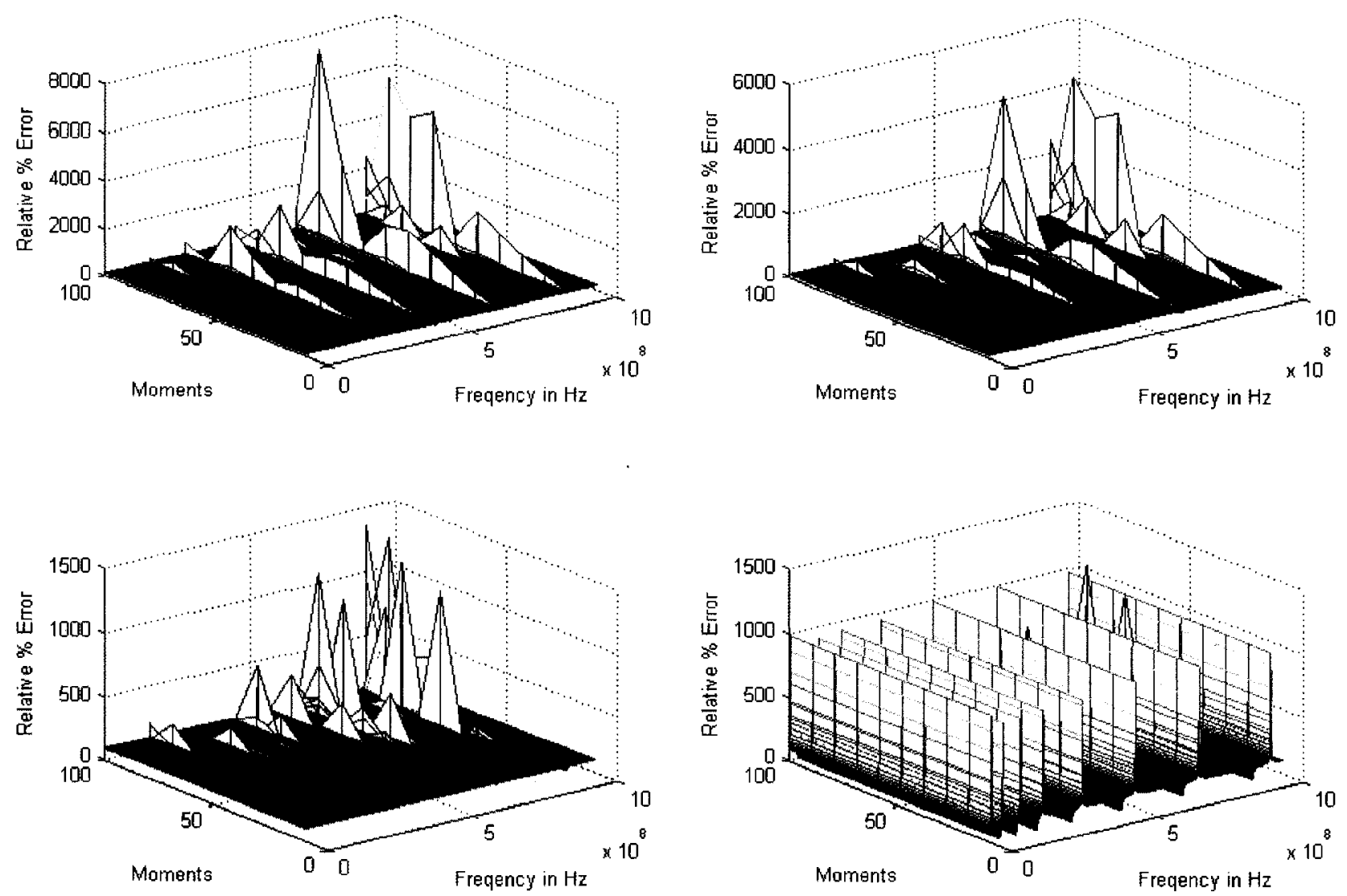

Figure 5.20: Relative Percentage Error of the Frequency Domain Response for a SUGAR Model with 500 elements using SOAR 
Discussion The SOAR procedure had a greater inaccuracy with the 150-element SUGAR model, but still provided a good approximation to the full response at lower damping. However, the 500-element model only provided a good approximation at very low damping. Hence, an any significant increase in the damping, resulting in no acceptable match to the full response. This was a result of the ill-conditioning of the original SUGAR matrices. Smaller models created with SUGAR have resulting matrices in which the entries are of the same order but as larger models are created the greater differences in the magnitude of the matrix entries result in a rapidly growing ill-conditioning issue that causes matrix operations, such as finding the inverse, becoming singular and, therefore, inaccurate.

\subsubsection{Conclusion on Frequency Response of SUGAR Models}

The frequency response analysis of models using SUGAR demonstrated that the Arnoldi algorithm was better suited for this problem, as the ill-conditioning problem found in modeling with SUGAR proved too difficult for SOAR to handle.

\subsection{Conclusion}

For further consideration, the frequency responses for the 540-node and 2700-node cantilevers are considered further in Appendix C. In addition a medium 1350-node cantilever is also presented.

The frequency domain analysis performed on the reduced FEM models demonstrated that both Arnoldi and SOAR performed about the same, within reasonable relative percentage error threshold limits for the different levels of damping that were examined. Even at higher damping, 
the overall performance of both SOAR and the Arnoldi algorithm were comparable. At higher frequencies, there was more error present but this error can be minimized by increasing the number of moments taken to approximate the full model.

It was also observed that the randomly chosen companion form for the second-order system being modeled using the Arnoldi algorithm did have a good match to the full model at a much lower frequency range and at lower damping. This companion form became more inaccurate as the damping approached critical damping.

The SUGAR models performed best using the Arnoldi algorithm for implementing model reduction. The SOAR procedure introduced a much larger amount of error in the reduced system and it was noted that the matrices generated by SUGAR were highly ill-conditioned, on the order of $10^{19}$. This ill-conditioning had a greater effect in the implementation of the SOAR algorithm because scaling the matrices had no effect in resolving this issue. However, the Arnoldi algorithm uses a companion form of the second-order system which reduces the illconditioning as the mass, damping and stiffness need not be scaled directly but rather by a scaled identity matrix that results in the companion form, refer to equation 3.7.2. This reduced the illconditioning problem by a magnitude of $10^{12}$, making the problem much better conditioned.

In this chapter, the frequency response was considered extensively and found that the frequency domain analysis of models generated using FEM and reduced using both SOAR and Arnoldi performed equally well, but models generated using SUGAR performed much better using the Arnoldi algorithm.

The time domain analysis will now be investigated with the same methodology employed. 


\section{Chapter 6}

\section{Time Domain Analysis of Krylov Based Model Reduction Techniques}

Here the theoretical transient response of a cantilever beam will be considered as a verification tool for the response of the cantilever modeled with finite element methods (FEM), and SUGAR.

A precise numerical match is not expected, as even analytically, the response of the cantilever will still be approximated at some stage since the total theoretical response is summed over $n$ roots and $n$ can range from 1 to infinity.

The general equation that governs the cantilever beam is as follows

$$
E_{m} I_{m} \frac{\partial^{4} z(x, t)}{\partial x^{4}}+m \frac{\partial^{2} z(x, t)}{\partial t^{2}}=y(x, t)
$$

For a beam with uniform cross-section,

$$
\begin{gathered}
\frac{\partial^{4} X(x)}{\partial x^{4}}-k^{4} X=0 \\
k^{2}=\sqrt{\frac{\hat{\omega}^{2} m}{E_{m} I_{m}}}
\end{gathered}
$$


where $E_{m}$ is the Young's modulus, $I_{m}$ is the moment of inertial, $m$ is the mass of the beam.

The equation of motion for a cantilever is as follows

$$
\begin{gathered}
M_{i} \ddot{z}_{i}+D_{i} \dot{z}_{i}+K_{i} z_{i}=P_{i} \\
\varsigma_{i}=\frac{D_{i}}{2 M_{i} \omega_{i}}=\frac{D_{i}}{D_{c r}}
\end{gathered}
$$

where $M, D$, are $K$ are the mass, damping, and stiffness matrix respectively.

The critical damping of the analytical system is given as

$$
D_{c r}=2 \sqrt{M_{i j} K_{i j}}
$$

The analytical response of a cantilever is given as,

$$
\begin{aligned}
& w_{n} x(t)=\text { force } * \frac{2 L^{3}}{\operatorname{root}^{4} * E_{m} * I_{m}} * \frac{\left[-\cos \left(k_{n} L\right) \sinh \left(k_{n} L\right)+\sin \left(k_{n} L\right) \cosh \left(k_{n} L\right)\right]}{\left[\sin \left(k_{n} L\right)-\sinh \left(k_{n} L\right)\right]} * \\
& \left(1-e^{-\zeta_{n} \omega_{n} t}\left[\cos \omega_{n} \sqrt{1-\varsigma_{n}^{2}}(t)+\frac{\varsigma_{n} \omega_{n}}{\omega_{n} \sqrt{\left(1-\varsigma_{n}^{2}\right)}} \sin \omega_{n} \sqrt{1-\varsigma_{n}^{2}}(t)\right]\right)
\end{aligned}
$$

where $r o o t=k_{n} L$ of the system, force is the excitation force and $\omega_{n}=k_{n}^{2} \sqrt{\frac{E_{m n} I_{m}}{M_{n}}}$

A detailed proof and explanation of terms are provided in Appendix A.

For the purposes of this thesis, the analytical cantilever was used to verify the steady-state value of the cantilever and the general overall expected response. It was not expected that the MEMS models created, using FEM or SUGAR, would be able to match the analytical response exactly as the boundary conditions imposed on the FEM models at the fixed end were very constraining and not a true reflection of a real-life situation, but the analytical response could be used as a gauge to determine the main characteristics of the response to varying the damping and the steady-state value. 


\subsection{Error Analysis}

The error presented in the numerical analysis of the cantilever is relative error plotted on a $\log _{10}$ scale for easier comprehensibility. As there are no set limits for acceptable error for model reduction, the aim, again, will be to have minimum error.

The relative error, $\delta x$ is found as follows

$$
\delta x=\frac{x_{0}-x}{x}
$$

Relative error is unitless.

\subsection{FEM Cantilever Model}

Similar to the frequency domain analysis, for a complete and effective comparison of modeling techniques and model reduction algorithms as possible, one of the simplest mechanical structures is modeled using FEM with different model sizes: 540-nodes , 1350-nodes and 2700nodes. The response of the 1350-node model will examined in Appendix D.

The goal of using three (small, medium and large) models was to observe the behavior of both the Arnoldi algorithm and the SOAR algorithm for a range of parameters. In addition to the model size, the effect of damping as well as the number of moments used will be compared for both types of model reduction techniques. 


\subsubsection{Cantilever Models with Damping $=0 \times M$}

At no damping, the system matrices for both companion forms for Arnoldi are identical and are shown below,

$$
\underbrace{\left[\begin{array}{cc}
M & 0 \\
0 & I
\end{array}\right]}_{A}\left[\begin{array}{c}
\ddot{u}(t) \\
\dot{u}(t)
\end{array}\right]+\underbrace{\left[\begin{array}{cc}
0 & K \\
-I & 0
\end{array}\right]}_{B}\left[\begin{array}{c}
\dot{u}(t) \\
u(t)
\end{array}\right]=\underbrace{\left[\begin{array}{c}
F(t) \\
0
\end{array}\right]}_{E}, y(t)=\left[\begin{array}{ll}
0 & L
\end{array}\right]\left[\begin{array}{c}
\dot{u}(t) \\
u(t)
\end{array}\right]
$$

\section{Small Cantilever Built with 540 nodes}

The condition numbers for the reduced order matrices for both configurations of the companion form for the Arnoldi Algorithm were found for the full range of damping $\left(1,0,10^{-1}, 10^{-2}, 10^{-3}\right.$, $10^{-4}$ and $10^{-5}$ ) and for all moment intervals (10 to 150 ). They were all in the range of $10^{4}$ to $10^{5}$, indicating that the matrices were conditioned within an acceptable range, therefore eliminating the premise that ill-conditioning might be responsible for any instabilities in the time domain response.

As an initial example, the time domain response of a small beam with only 540 nodes will be considered. Three model reductions, two different companion forms of the Arnoldi algorithm and the SOAR procedure will be examined.

The time domain response comparison of the full system, the Arnoldi reduced systems, and the SOAR reduced system is shown below in Figure 6.1 and was observed to match the response of the full system within an acceptable limit. There was an obvious difference in the full response and the reduced model responses. This was a result of a slight shift in phase when model reduction was performed for the small model, so even at moments $\geq 150$, the response remained the same as the reduced models with only 10 moments. 


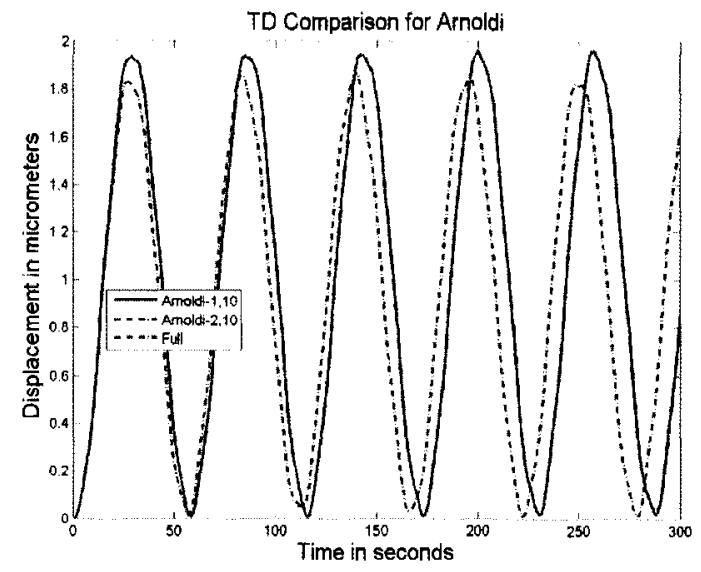

(a) with Arnoldi

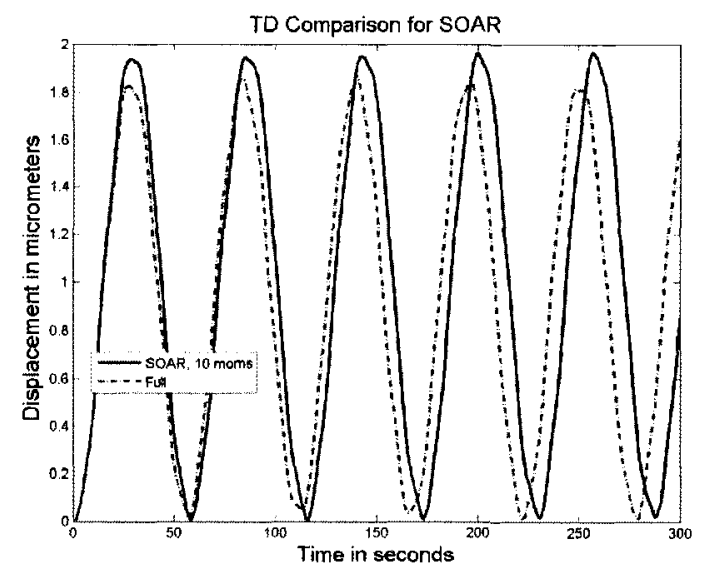

(b) with SOAR

Figure 6.1: Comparison of time domain response for the full system and reduced models of a 540-node Cantilever with Damping $=0 \times M$

Discussion Figure 6.2 illustrates the relative error of performing model reduction using the Arnoldi and the SOAR algorithms and it was shown that, despite the notable difference in the comparison between the full and the reduced models, the relative errors for all three model reductions were comparable. As stated previously, the difference between the full and all the reduced models for the 540-node cantilever stemmed from a slight phase shift and led to much higher relative error between the full and reduced systems. In 
further analysis, it will be shown that as the size of the model increased this phase shift disappeared. Additionally, the large periodic error, due to the phase error that was seen in Figure 6.2, diminished in larger models as the number of moments taken was increased. 

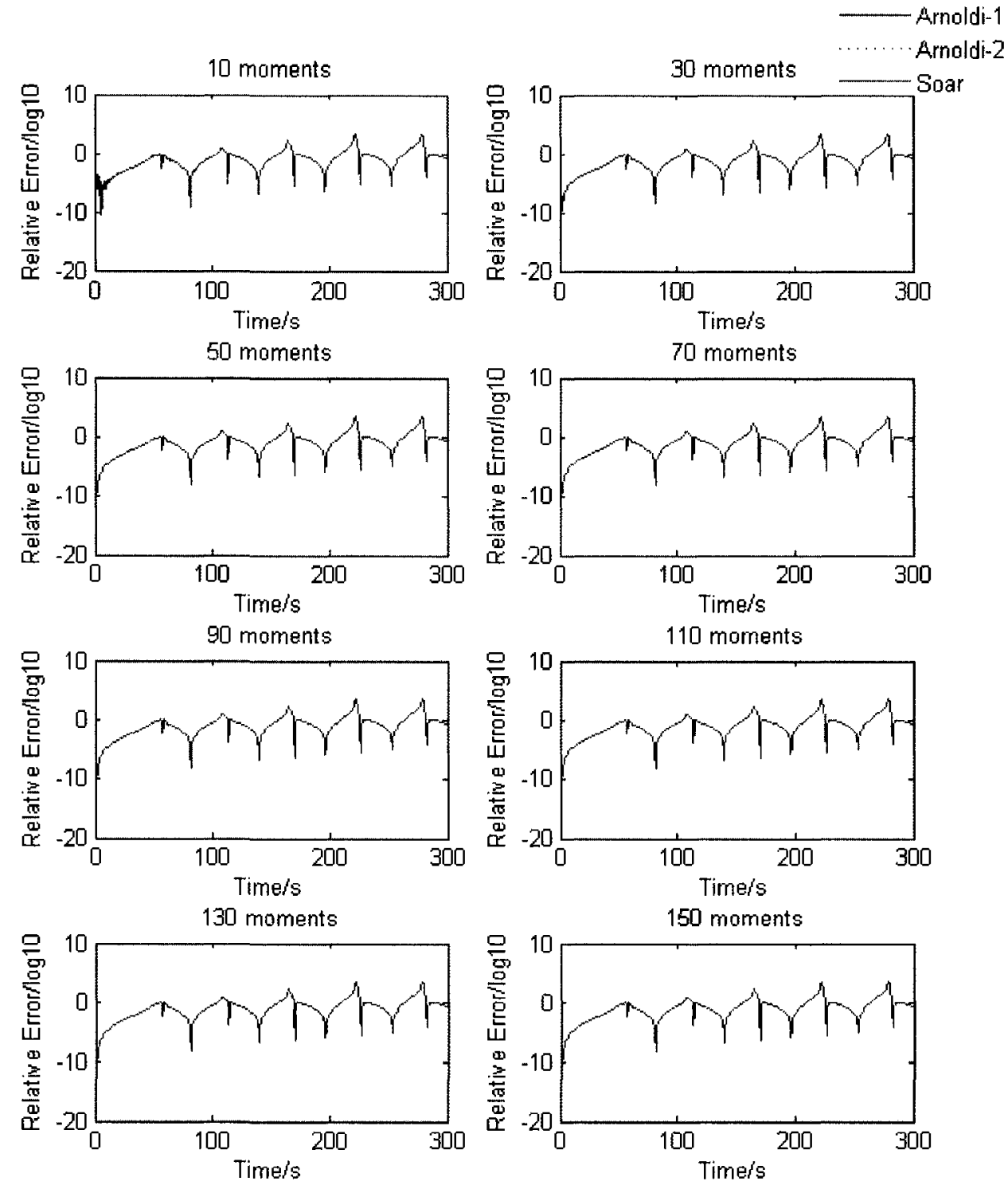

Figure 6.2: Relative Error for 540-node cantilever using Arnoldi at Damping $=0 \times M$ 


\section{Large Cantilever Built with 2700 nodes}

A large beam with 2700-nodes will now be evaluated in the time domain for the full system, the Arnoldi reduced system, and the SOAR reduced system.

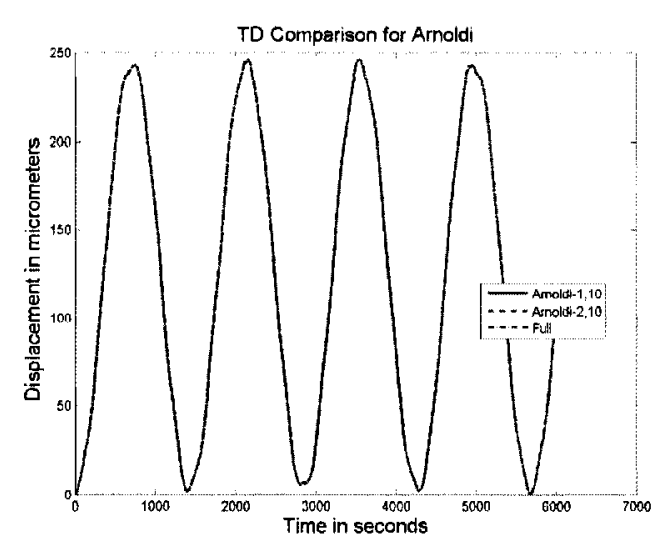

(a) with Arnoldi

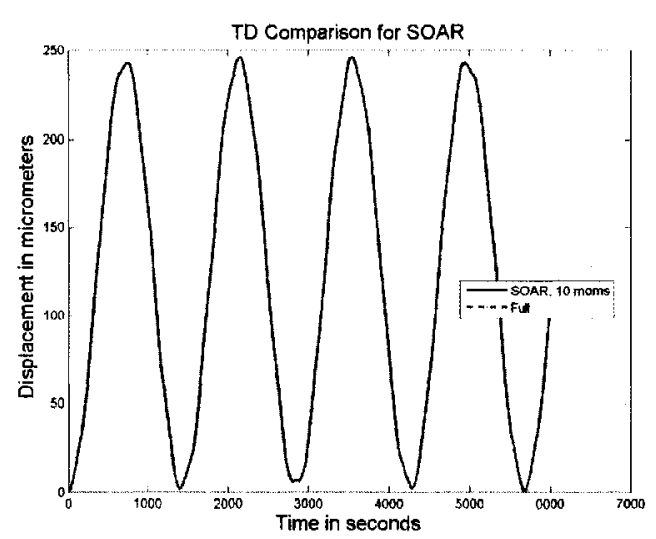

(b) with SOAR

Figure 6.3: Comparison of time domain response for the full system and reduced models of a 2700-node Cantilever with Damping $=0 \times M$

Discussion Figure 6.3 shows that there were no discernible differences in the reduced and full time domain responses for both Arnoldi and SOAR with respect to the large 2700node beam at 10 moments. However, on closer inspection of the relative errors of the three systems, it was observed that SOAR had consistently lower error for the range of moments considered.

The overall magnitude of the relative error must be taken into account as well and even at 10 moments, the error was so insignificant that the benefit of using the SOAR model reduction over Arnoldi was non-existent with no damping as shown in Figure 6.4.

The response for the larger model also showed that the phase shift shown in the time domain response for the small 540-node cantilever model was not present for the larger 
2700-node model. Also, the results for the 1350-node model shown in Appendix D, also ilustrated no phase-shift. The periodicity of the relative error also diminished as the number of moments increased. 


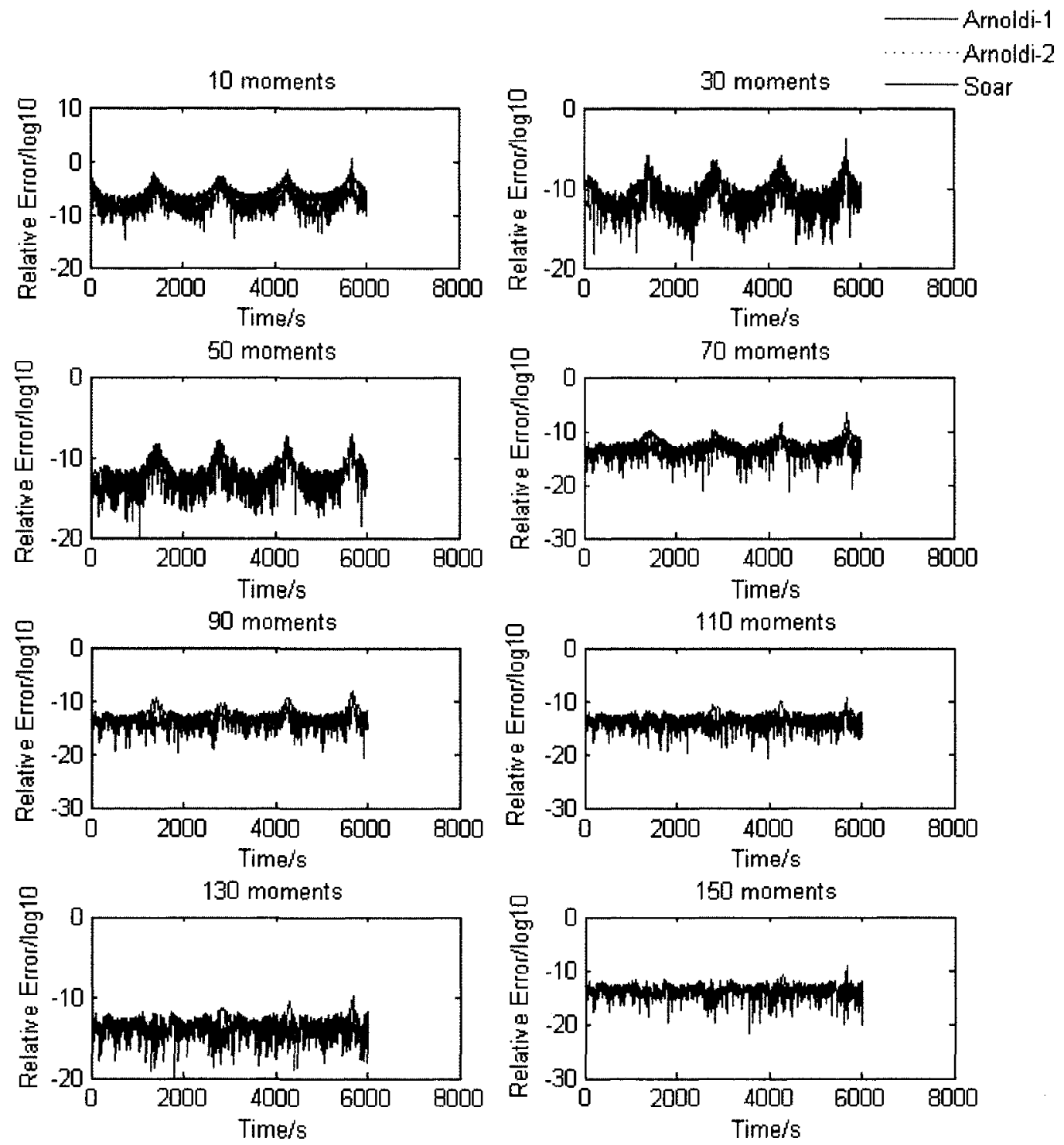

Figure 6.4: Relative Error for 2700-node cantilever using Arnoldi at Damping $=0 \times M$ 


\subsubsection{Cantilever Models with Damping $=0.01 \times M$}

The Damping of the system is now set such that $D=0.01 \times M$, and the same comparisons were performed. The system matrices for the two stable Arnoldi configurations are now

$$
\underbrace{\left[\begin{array}{cc}
M & 0.01 * M \\
0 & I
\end{array}\right]}_{A}\left[\begin{array}{c}
\ddot{u}(t) \\
\dot{u}(t)
\end{array}\right]+\underbrace{\left[\begin{array}{cc}
0 & K \\
-I & 0
\end{array}\right]}_{B}\left[\begin{array}{c}
\dot{u}(t) \\
u(t)
\end{array}\right]=\underbrace{\left[\begin{array}{c}
F(t) \\
0
\end{array}\right]}_{E}, y(t)=\left[\begin{array}{ll}
0 & L]
\end{array}\right]\left[\begin{array}{c}
\dot{u}(t) \\
u(t)
\end{array}\right]
$$

and

$$
\underbrace{\left[\begin{array}{cc}
M & 0 \\
0 & I
\end{array}\right]}_{A}\left[\begin{array}{c}
\ddot{u}(t) \\
\dot{u}(t)
\end{array}\right]+\underbrace{\left[\begin{array}{cc}
0.01 * M & K \\
-I & 0
\end{array}\right]}_{B}\left[\begin{array}{c}
\dot{u}(t) \\
u(t)
\end{array}\right]=\underbrace{\left[\begin{array}{c}
F(t) \\
0
\end{array}\right]}_{E}, y(t)=\left[\begin{array}{ll}
0 & L
\end{array}\right]\left[\begin{array}{c}
\dot{u}(t) \\
u(t)
\end{array}\right]
$$

Small Cantilever Built with 540-nodes

Again, the time domain response for the full system, the Arnoldi reduced system, and the SOAR reduced system will be considered, now with a damping of $D=10^{-2} \times M$.

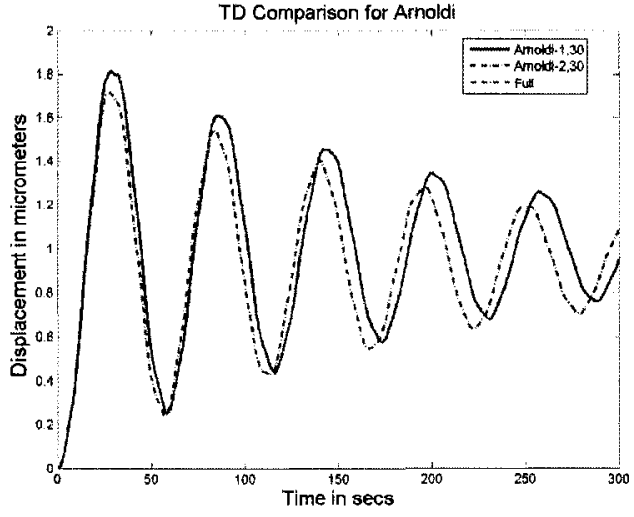

(a) with Arnoldi, 30moms

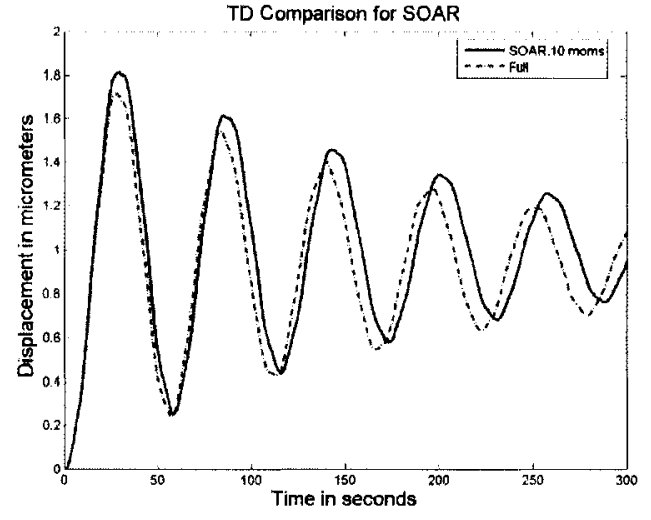

(b) with SOAR, 10 moms

Figure 6.5: Comparison of time domain response for the full system and reduced models of a 540-node Cantilever with Damping $=10^{-2} \times M$ 
Discussion For the small cantilever of 540-nodes, both model reductions gave stable responses at 10 moments as shown in Figure 6.5. However, on examination of the the relative errors of Figure 6.6, and it was seen that there was some instability in the Arnoldi model reduction at 70 moments although it matched the full model at lower moments. SOAR remained stable and with very low relative error at 10 moments and all other moments. 

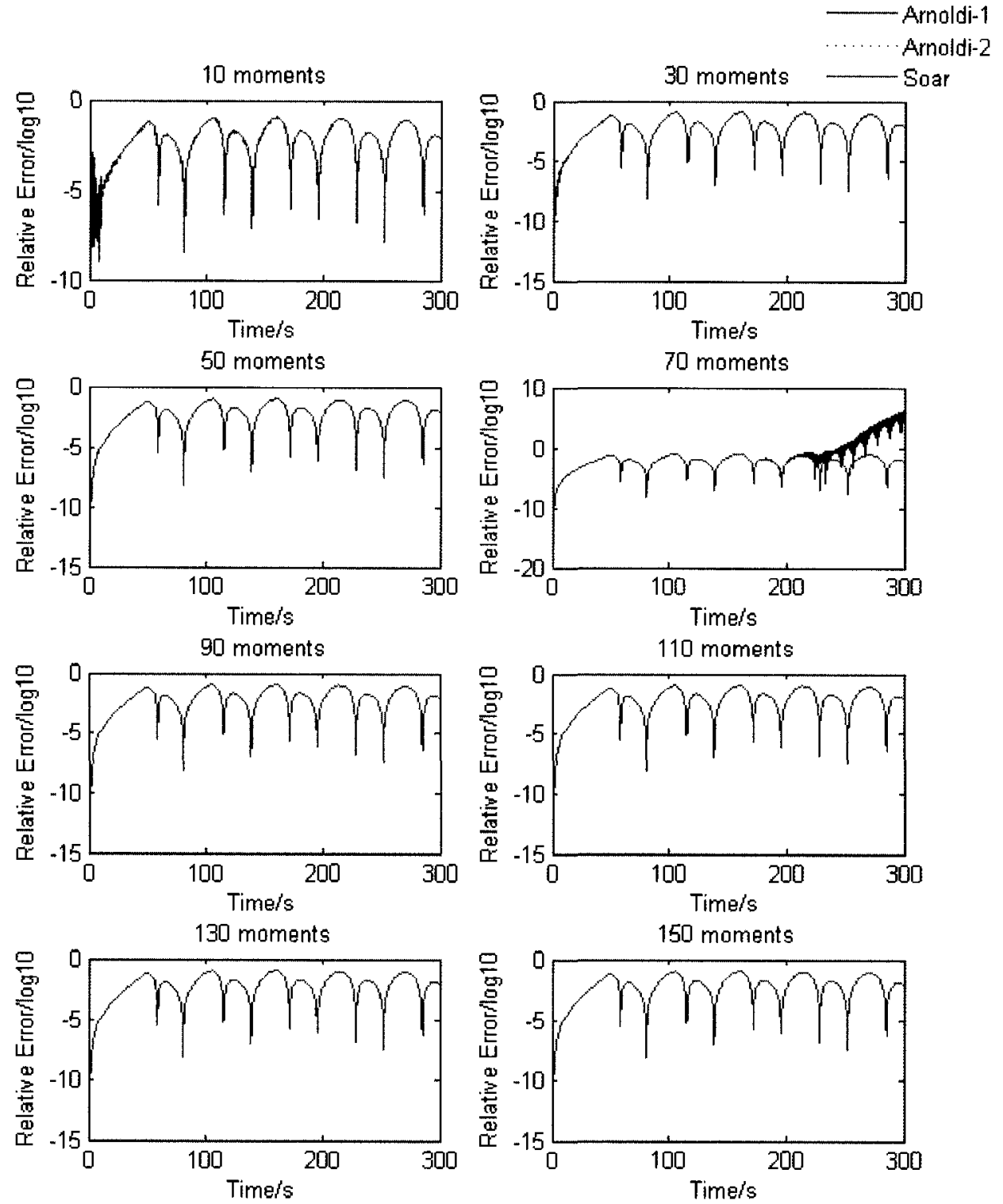

Figure 6.6: Relative Error for 540-node cantilever using Arnoldi at Damping $=10^{-2} \times M$ 


\section{Large Cantilever Built with 2700-nodes}

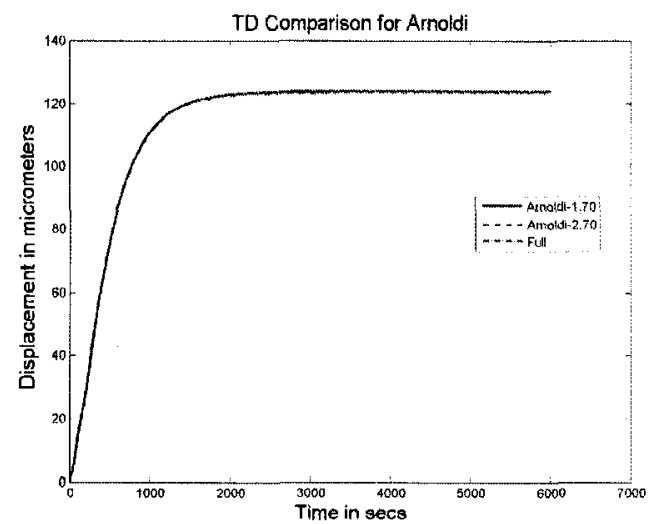

(a) with Arnoldi, with 70 moms

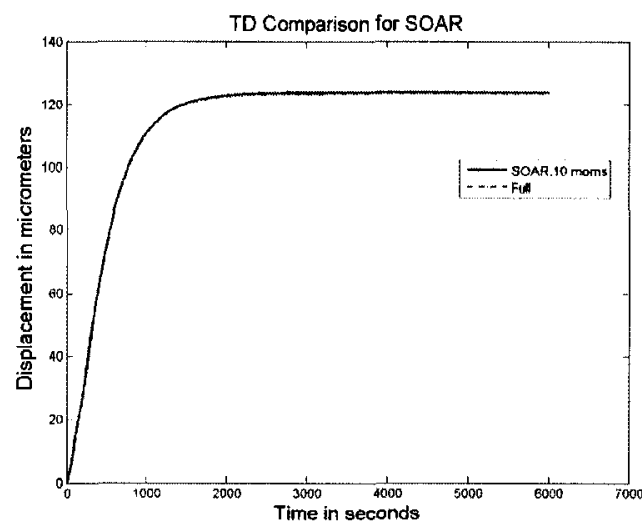

(b) with SOAR, with 10 moms

Figure 6.7: Comparison of time domain response for the full system and reduced models of a 2700-node Cantilever with Damping $=10^{-2} \times M$

Discussion To achieve a stable response with the large cantilever of 2700-nodes, at least 110 moments were required for both companion forms using the Arnoldi algorithm as shown in Figure 6.7 while, even at 10 moments, the SOAR algorithm continues to give a good match to the full model.

The comparison of the relative errors of performing model reduction using the Arnoldi and the SOAR algorithm can be observed in Figure 6.8. It shows that a stable response was achieved for both forms of the Arnoldi algorithm at 70 moments but there was instability in the systems at 90 moments. All the time domain responses became stable at moments $\geq 110$. SOAR remained stable and with very low relative error at 10 moments. 

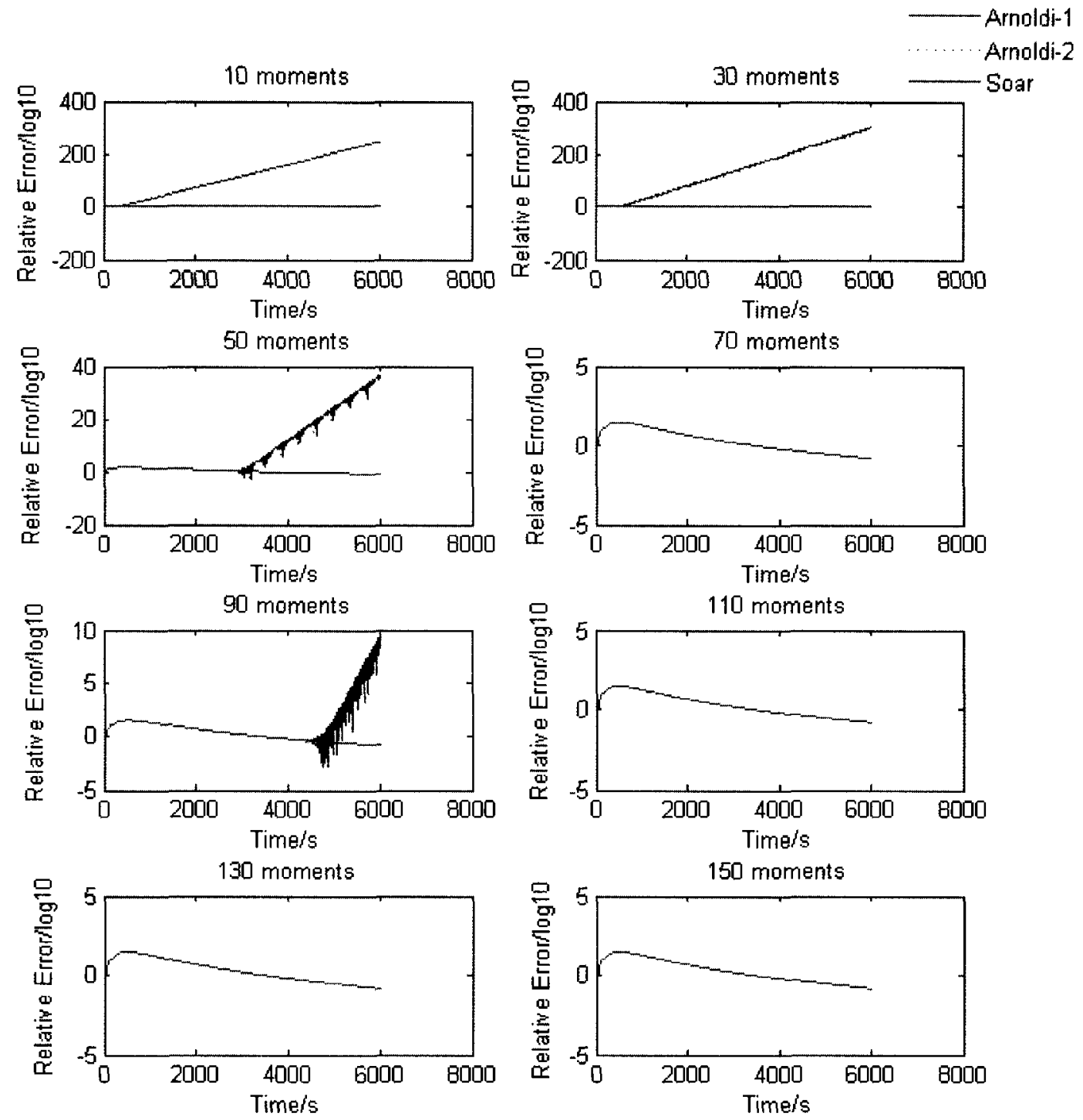

Figure 6.8: Relative Error for 2700-node cantilever using Arnoldi at Damping $=10^{-2} \times M$ 


\subsubsection{Cantilever Models with Damping $=10^{-1} \times M$}

The damping of the system is now set such that $D=10^{-1} \times M$, and the same comparisons were performed. The system matrices for the two stable Arnoldi configurations are now

$$
\underbrace{\left[\begin{array}{cc}
M & 10^{-1} * M \\
0 & I
\end{array}\right]}_{A}\left[\begin{array}{c}
\ddot{u}(t) \\
\dot{u}(t)
\end{array}\right]+\underbrace{\left[\begin{array}{cc}
0 & K \\
-I & 0
\end{array}\right]}_{B}\left[\begin{array}{c}
\dot{u}(t) \\
u(t)
\end{array}\right]=\underbrace{\left[\begin{array}{c}
F(t) \\
0
\end{array}\right]}_{E}, y(t)=\left[\begin{array}{ll}
0 & L
\end{array}\right]\left[\begin{array}{c}
\dot{u}(t) \\
u(t)
\end{array}\right]
$$

and

$$
\underbrace{\left[\begin{array}{cc}
M & 0 \\
0 & I
\end{array}\right]}_{A}\left[\begin{array}{c}
\ddot{u}(t) \\
\dot{u}(t)
\end{array}\right]+\underbrace{\left[\begin{array}{cc}
10^{-1} * M & K \\
-I & 0
\end{array}\right]}_{B}\left[\begin{array}{c}
\dot{u}(t) \\
u(t)
\end{array}\right]=\underbrace{\left[\begin{array}{c}
F(t) \\
0
\end{array}\right]}_{E}, y(t)=\left[\begin{array}{ll}
0 & L
\end{array}\right]\left[\begin{array}{c}
\dot{u}(t) \\
u(t)
\end{array}\right]
$$

\section{Small Cantilever Built with 540 nodes}

The time domain responses for the full system, the Arnoldi reduced systems, and the SOAR reduced system, are shown in Figure 6.9

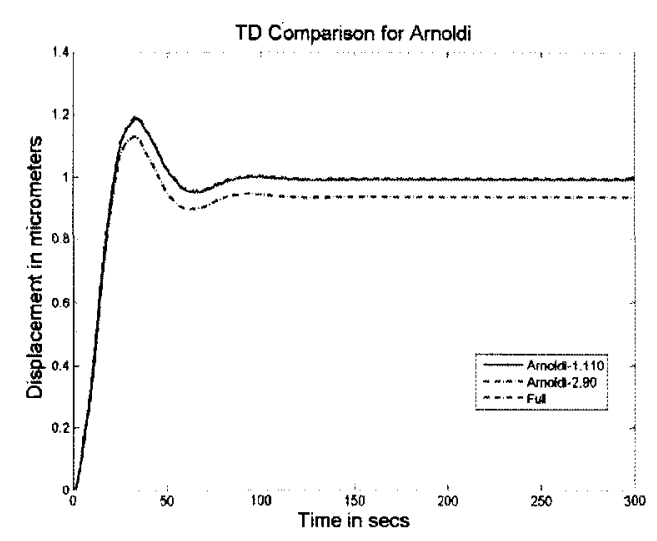

(a) with Arnoldi, with 30 and 70 moms

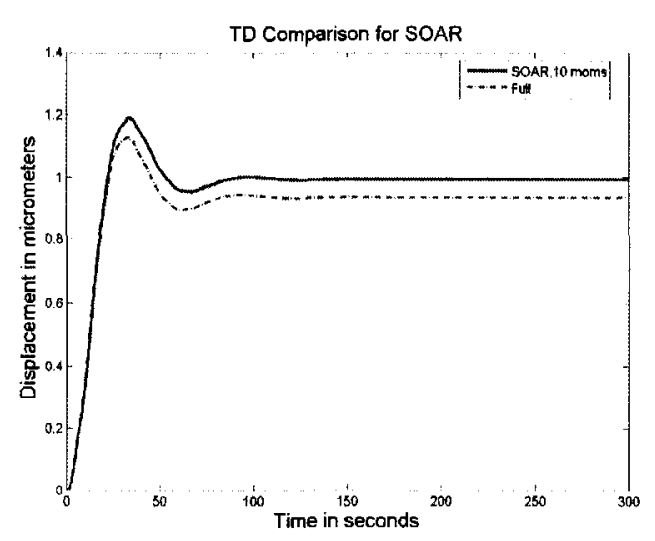

(b) with SOAR

Figure 6.9: Comparison of time domain response for the full system and reduced systems of a 540-node Cantilever with Damping $=10^{-1} \times M$ 
Discussion A stable time domain response in using both Arnoldi forms was achieved with 110 moments as seen in Figure 6.9. All the time domain analysis performed with Arnoldi below 110 moments gave highly unstable results. The relative error of the model reductions as shown in Figure 6.10 also illustrates that the model reduction performed with the Arnoldi algorithm required at least 110 moments with both companion form configurations. SOAR continued to demonstrate sufficiently small error for the 540 -node cantilever model at 10 moments, Figure 6.9(b). 

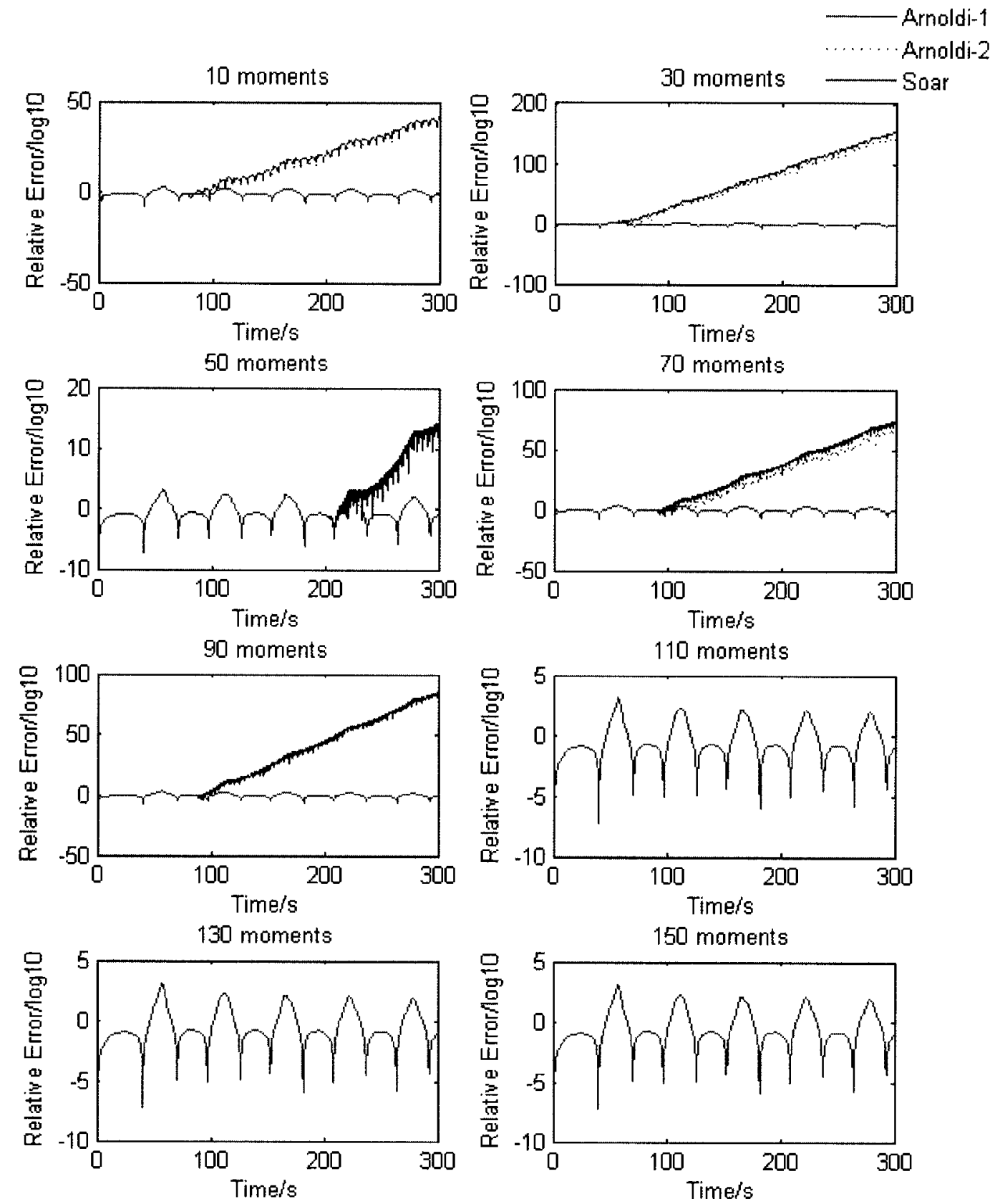

Figure 6.10: Relative Error for 540-node cantilever using Arnoldi at Damping $=10^{-1} \times M$ 


\section{Large Cantilever Built with 2700-nodes}

The time domain responses for the full 2700-node system, the Arnoldi reduced systems, and the SOAR reduced system, are shown in Figure 6.11.

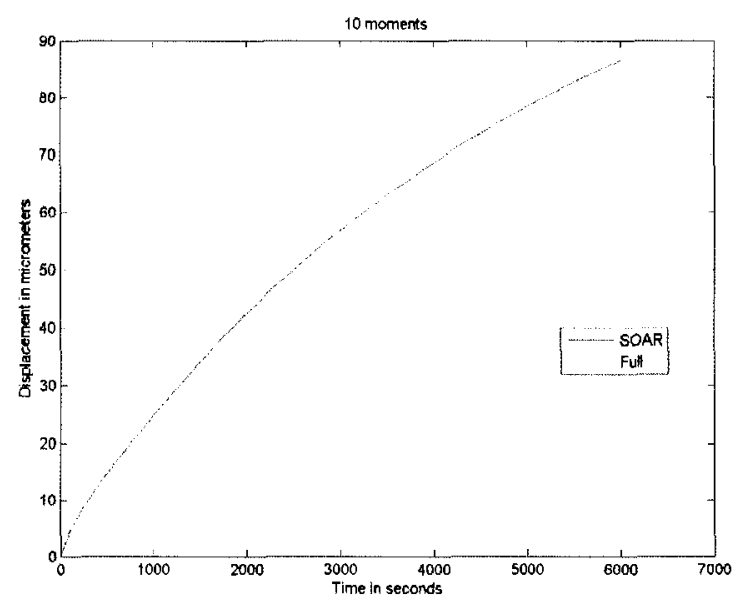

Figure 6.11: Comparison of time domain response for the full system and SOAR reduced model of a 2700 -node Cantilever with Damping $=10^{-1} \times M$, with 10 moments

Discussion Again, both companion forms for Arnoldi could not achieve a stable response at 150 moments, whereas SOAR continued to demonstrate sufficiently small error for the 2700 -node cantilever model even at 10 moments.

\subsubsection{Conclusion on the Time Domain Analysis of FEM models}

The time domain analysis of the 540-node, 1350-node (see Appendix D), and the 2700-node FEM model revealed that as the damping of the systems approached critical damping, the Arnoldi algorithm became highly unstable. At damping values lower than $D_{c r}=2 \sqrt{M_{i j} K_{i j}}$ or $0<\xi<1$, Arnoldi performed comparable to SOAR. In addition, as model size increased, the number of moments needed to be taken to achieve a stable response increased as well, which 
was as expected.

\subsection{SUGAR Models}

The time domain analysis of models created using the SUGAR modeling tool will now be considered for two models: the first with 150 elements and the second with 500 elements.

The value of the damping used in SUGAR is set to a range of $\left(10^{15}\right.$ to $\left.10^{19}\right) \times D$. The difference in defining the magnitude of the damping used for SUGAR lies in using the damping matrix SUGAR generates, not a direct scaling of the mass matrix, $M$, as was done in FEM. However, it should be noted that the resulting damping matrix is still a factor of the mass matrix, so it still satisfies the proportional Rayleigh damping criteria for ensuring that an orthogonal transformation can be applied via Krylov subspace based model reduction techniques.

MEMS modeled using SUGAR were made as simple as possible by basically creating 150 or 500 beam elements linked together and fixed at one end, much like a regular cantilever beam. The resulting sizes of the number of nodes were 450 and 1500 respectively.

As with the FEM models, model reduction on the SUGAR models was realized using both the Arnoldi algorithm and the SOAR procedure.

\subsubsection{Numerical Analysis of SUGAR Models}

The analysis of both SUGAR models using the Arnoldi algorithm and the SOAR procedure proved to be much less robust than the FEM model. In order to achieve any working results with the Arnoldi algorithm the models had to be scaled; however, with SOAR, stable results could only be achieved when there was little or no damping present. 
The preconditioning of the matrices through scaling was a direct result of ill-conditioning problems that result in modeling with SUGAR. Considering the 150 element model, the original condition number of the model was found to be on the order of $>10^{21}$ for the companion form needed for the Arnoldi algorithm, but with scaling this was brought down to $\approx 10^{8}$. However, the original matrices used in the SOAR procedure maintained a condition number on the order of $10^{19}$ or larger, with or without scaling. The developer of SUGAR used models that were $\leq 40$ elements in size, and had condition numbers of $10^{9}$, which did not present the ill-conditioning issue that arose in the much larger models that were tested.

Figures 6.12 and 6.13 illustrate the time domain response for both SUGAR models. These results demonstrated a good match between the full model and reduced models using Arnoldi. This was further evident by the low relative error achieved, on the order of $10^{-} 5$, at only 10 moments, as shown in Figures 6.14 and 6.15 .

However, the effect of damping on the stability of the response is clearly demonstrated here for both Arnoldi and SOAR. As stated previously, stable results could only be achieved using SOAR with no damping and with Arnoldi. Also, as the damping approached critical damping, $\xi=1$, the response becomes unstable. 

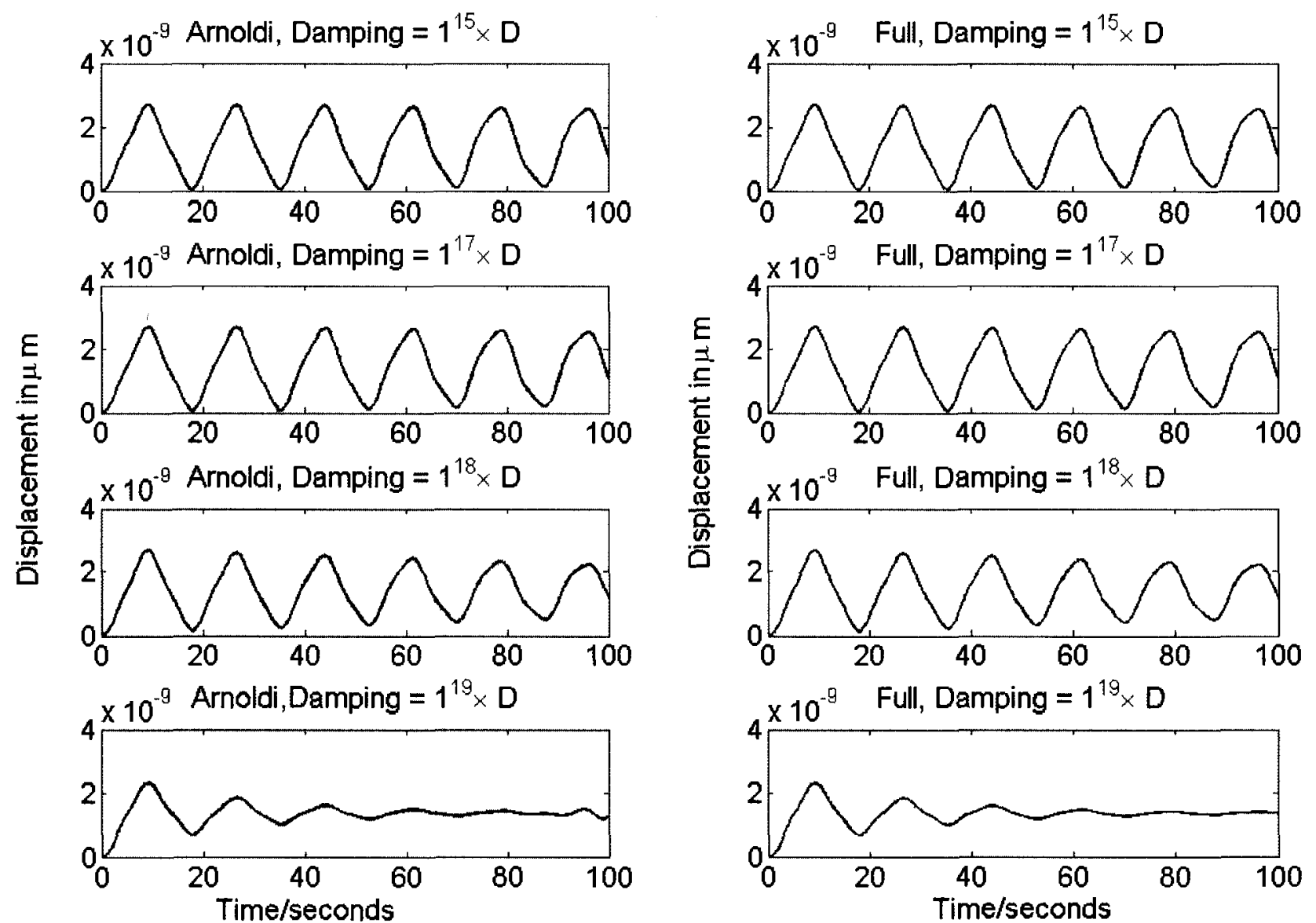

Figure 6.12: Time Domain Response for a SUGAR Model with 150-elements 

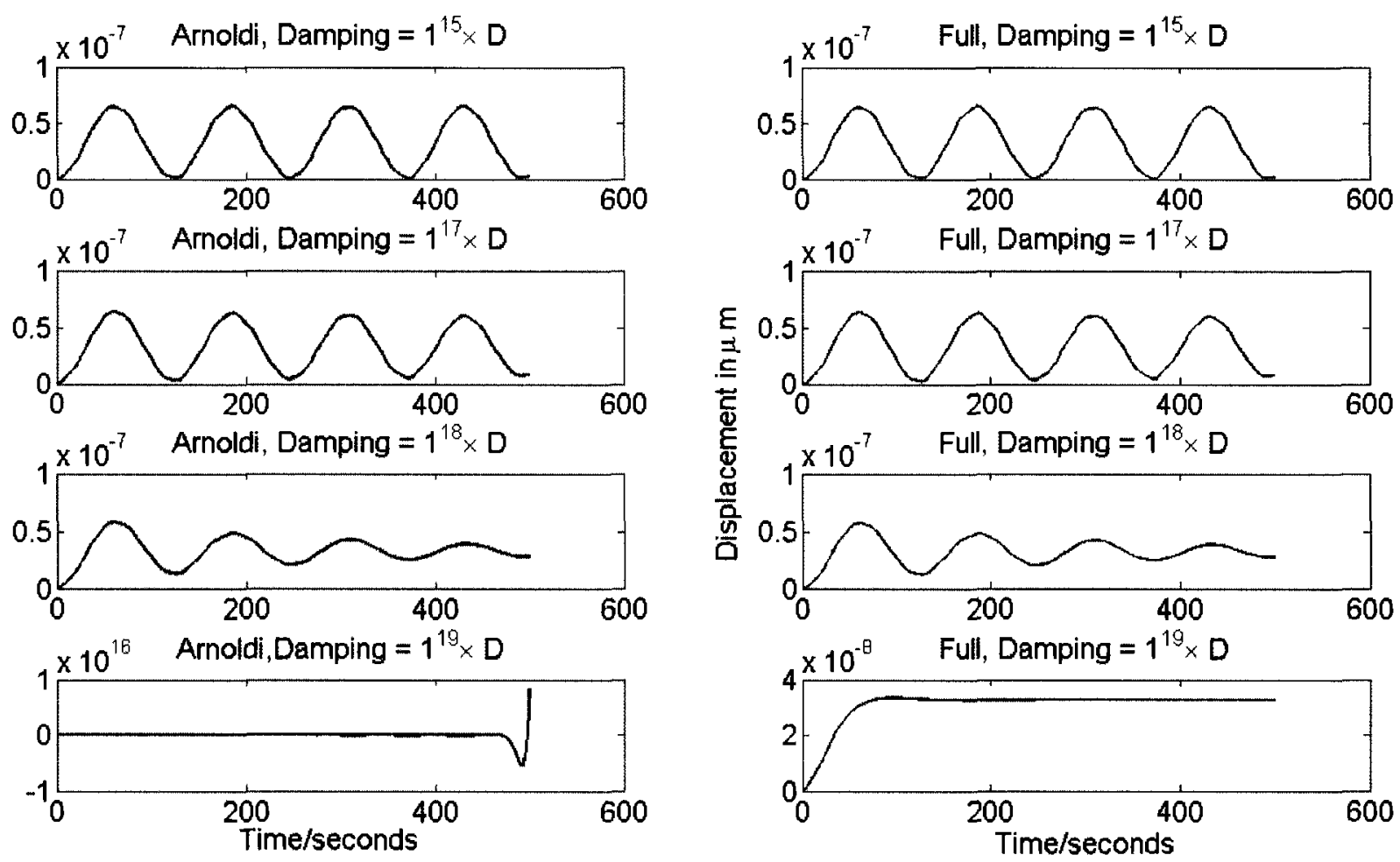

Figure 6.13: Time Domain Response for a SUGAR Model with 500-elements 

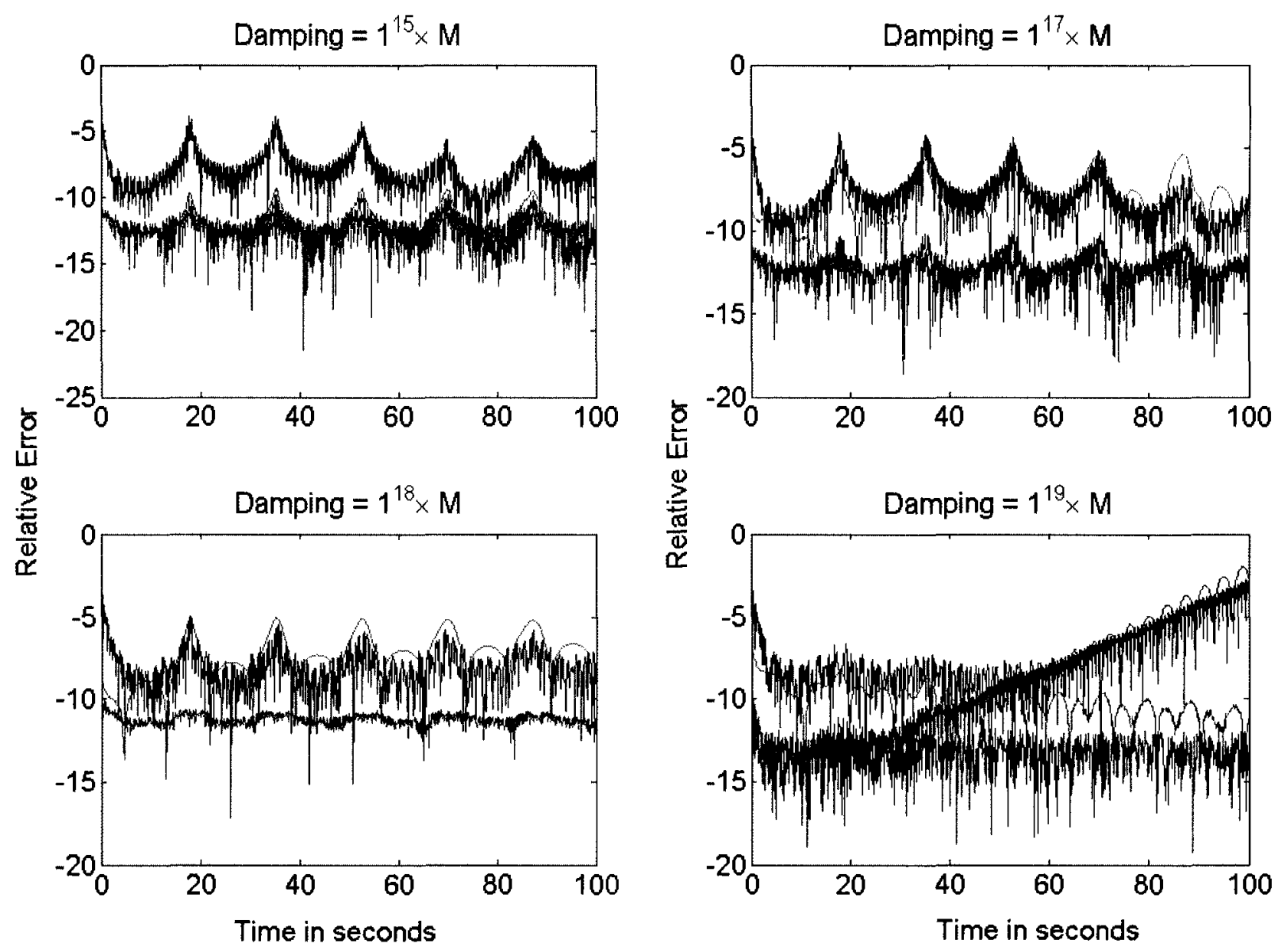

Figure 6.14: Relative Error of the Time Domain Response for a SUGAR Model with 150elements 

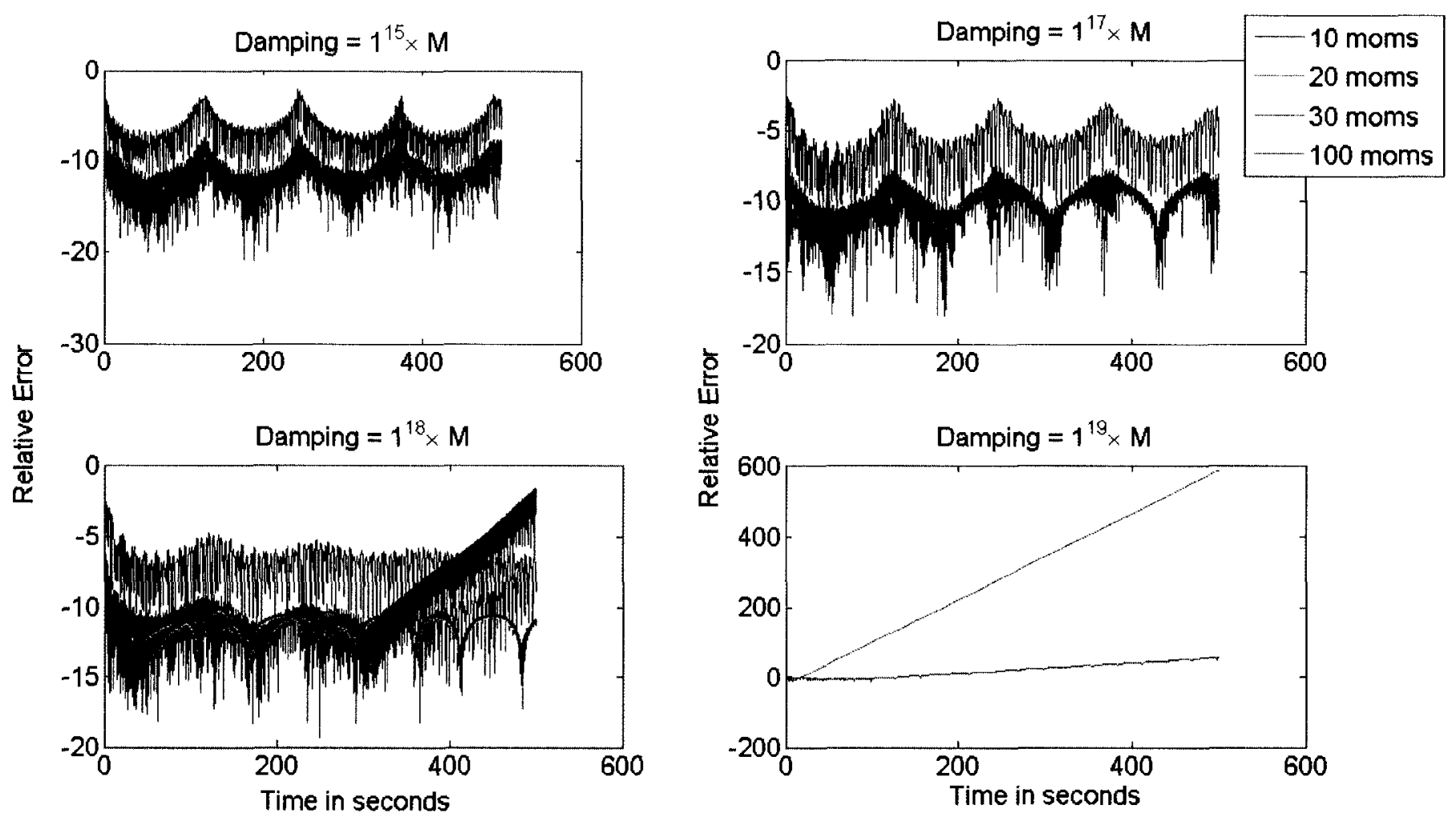

Figure 6.15: Relative Error of the Time Domain Response for a SUGAR Model with 500elements 


\section{Conclusion on the Time Domain Analysis of SUGAR Models}

It is evident from the results achieved using both the Arnoldi algorithm and the SOAR procedure for performing model reduction on SUGAR models, that only the Arnoldi algorithm was realistic in achieving good results. The literature [9] suggested using SOAR for implementing model reduction but the models used were on the order of $N=32$. Frankly, at that scale and with the computational power available today, the analysis of models with those dimensions should be computationally trivial. In the above discussion, more realistic sized systems were used to generate the effectiveness of both model reduction techniques, and it was apparent that only the Arnoldi algorithm was capable of achieving good stable results for the purposes for which SUGAR was designed. It was also clear that the Arnoldi algorithm was much better than SOAR in handling matrices that were initially very ill-conditioned.

In addition, the effect of damping on the stability of model reduction techniques was further emphasized in the results achieved for model reduction of SUGAR models. Again, it was found that as the damping approached critical damping, the response for reduced models, in this case the Arnoldi algorithm, became highly unstable. 


\subsection{Conclusion}

The time domain analysis provided a much clearer view of the stability of the response of the reduced systems. It was seen that, while researchers have chosen to focus on the frequency domain analysis when performing model reduction on most types of mathematical modeling, the results in performing the time domain analysis provided evidence that it should be more carefully considered to ensure that the reduced modeled structure or device provides a stable representation of the original system. This was reinforced by the results obtained for the frequency domain analysis which showed that both SOAR and Arnoldi performed comparably for the FEM models but where the Arnoldi algorithm produced reduced models that were unstable in the time domain.

For FEM models, SOAR outperformed the Arnoldi algorithm, except when the damping approached zero. At zero damping or very close to zero damping, both the Arnoldi and SOAR model reduction techniques provided excellent matches to the full model with a minimum number of moments. However, as the damping increased and approached critical damping, it became clear that the Arnoldi algorithm generated reduced order models that were unstable, and a greater number of moments were needed at times to achieve a stable time domain response. It was also seen that at or above critical damping, i.e. $\xi \geq 1$, a stable response could not be achieved with Arnoldi, but the SOAR procedure continued to give excellent matched responses to the full model. Appendix D provides a larger range of time domain analysis for a medium cantilever with 1350-nodes and additional magnitudes of damping for the small and large cantilever presented above. It must be noted that the loss of stability in the reduced models created 
using the Arnoldi algorithm, as the damping approaches critical damping, occurred even for the two companion forms that passed the tests for ensuring stability and passivity.

In order to verify that this loss of stability occurred as a result of the system approaching critical damping, the applied damping of the system was compared to the theoretical critical damping for the cantilever given as

$$
D_{c r}=2 \sqrt{M_{i j} K_{i j}}
$$

It was found, in all the cantilevers modeled in FEM, the critical damping was approximately $D_{c r}=10^{-2} \times M$. This analytical result matched the numerical analysis performed on those models, and reaffirms that critical damping does cause instability in model reduction using the Arnoldi algorithm.

For the SUGAR models, SOAR failed to perform at any significant level of damping. As discussed above, the Arnoldi algorithm was able to match the full models with a low number of moments, but required scaling of the original system matrices to achieve these results. The problem with this requirement for scaling the matrices is that there is no clear mathematical science involved, but rather a trial and error procedure, so while Arnoldi clearly outperformed SOAR for SUGAR models, it still does not provide designers with a robust, precise tool. In addition, the Arnoldi algorithm was still susceptible to the amount of damping present in the original system and became unstable as critical damping is reached.

In conclusion, both the SOAR procedure and the Arnoldi algorithm have been shown to have situations where one was better suited than the other, but they faced instability issues that arose due to the inclusion of damping. Obviously, damping is an important factor and its impact on mathematical modeling should have greater consideration. 
The following chapter will take a closer look at the role of damping in model reduction techniques and suggest a more robust way to perform model reduction to avoid any arising instabilities due to damping in both the SOAR procedure and the Arnoldi algorithm. 


\section{Chapter 7}

\section{The Impact of Damping on Model Reduction}

\subsection{Motivation}

In the time domain analysis of FEM models, it was observed that, as the damping approached critical damping, model reduction using the Arnoldi algorithm became highly unstable. Once critical damping was achieved in the system, the models formed using the Arnoldi algorithm could not gain a stable response, even when the number of moments was increased.

This instability was also shown to be present in SOAR, but only in models created using SUGAR. In this case, as soon as there was any significant damping in the system, a stable response in the time domain could not be achieved. In addition, approaching critical damping caused the system to become unstable when model reduction was implemented using the Arnoldi algorithm and the modeling done in SUGAR, similar to the response of FEM models.

During the course of this research, no previous work done on model reduction which included an extensive numerical analysis on models with variable damping could be found. This 
could be partly due to the fact that damping is often excluded in mathematical modeling in order to capture essential features of a device that become more evident with no damping present. Also, as most mathematical modeling is often performed in the frequency domain, the influence of damping on the stability of mathematically modeled structures appears to be minimal and can be a root cause as to why researchers often choose to exclude it.

However, in the time domain analysis presented in Chapter 6, it was clearly demonstrated that damping does play an important role in mathematical modeling and should be considered. In this chapter, the contribution of damping on model reduction techniques will be considered, and the role it plays in the stability of models created with these techniques will be examined. Furthermore, in an attempt to eliminate the instabilities that can be present in model reduction techniques based on the Krylov subspace methods and increase the robustness of both the Arnoldi algorithm and the SOAR procedure, an alternative formulation to the orthogonal matrix, $Q$, is introduced and numerically examined. This formulation simplifies the model reduction process, is very robust and eliminates the need to generate more than one orthogonal matrix.

\subsection{The Contribution of Damping}

Damping is an inherent property of all systems, and whether it is externally applied, a component of the structural design of a device or a material property, it can play an essential role in modeling techniques as shown previously.

In order to evaluate the contribution of damping to the mechanical MEMS second-order system presented in this thesis, and represented by equation 7.2.1, the calculation of the moments of the second-order system will be closely examined. Recall that, the sum of the moments is 
equivalent to the pole or eigenvalue of a system.

The following outlines the procedure to find the moments of the system, and examines the effect that damping has on the magnitude of these moments.

The theoretical contribution of damping will first be considered, followed by a numerical analysis using a small FEM model as the basis for this evaluation.

\subsubsection{Moments of a Second Order System}

If the system defined in equation 3.7.1 is rewritten to exclude damping, it becomes

$$
\begin{aligned}
& M \ddot{z}(t)+K z(t)=F u(t) \\
& y=l^{T} z(t)
\end{aligned}
$$

and the corresponding transfer function is

$$
H(s)=l^{T}\left(s^{2} M+K\right)^{-1} F
$$

where $M \in \Re^{N \times N}, K \in \Re^{N \times N}, F \in \Re^{N}$ and $u \in C$

The poles of a system are given by

$$
Y(s)=\sum_{i}^{n} \frac{k_{i}}{s-\lambda_{i}}
$$

where $k_{i}$ are the zeros and $\lambda_{i}$ are the poles of the system.

By taking the Laplace transform and using a Taylor series expansion, the transfer function in equation 7.2.2 is expanded around $s=0$, the corresponding moments of the system are

$$
\begin{aligned}
& m_{0}=K^{-1} F \\
& m_{n}=\left(K^{-1} M\right)^{n-2} m_{n-2} \text { where } n \geq 1
\end{aligned}
$$

When model reduction via the Arnoldi algorithm is performed on the original system, the reduced model formed by the congruent transformation is 
$M_{\text {red }}=Q^{T} M Q, K_{\text {red }}=Q^{T} K Q, F_{\text {red }}=Q F$, and $l_{\text {red }}=Q l$

where $Q$ is the orthogonal matrix formed during formation of the Krylov subspace projection,

The reduced, undamped system is then given by

$$
\begin{aligned}
& M_{r e d} \ddot{z}(t)+K_{r e d} z(t)=F_{r e d} u(t) \\
& y=l_{r e d}^{T} z(t)
\end{aligned}
$$

and the corresponding transfer function is

$$
H(s)=l_{r e d}^{T}\left(s^{2} M_{r e d}+K_{r e d}\right)^{-1} F_{r e d}
$$

If damping is not excluded, the system maintains its original form as

$$
\begin{aligned}
& M \ddot{z}(t)+D \dot{z}(t)+K z(t)=F u(t) \\
& y=l^{T} z(t)
\end{aligned}
$$

then the corresponding transfer function is

$$
H(s)=l^{T}\left(s^{2} M+s D+K\right)^{-1} F
$$

Here, the moments of the system are not as clearly and easily defined as in equation 7.2.4 and are as follows: (see Appendix B for a complete proof),

$$
\begin{aligned}
m_{0} & =K^{-1} F \\
m_{1} & =\left(K^{-1} D\right) m_{0} \\
m_{n} & =\left(K^{-1} M\right) m_{n-2}-\left(K^{-1} D\right) m_{n-1} \text { where } n>1
\end{aligned}
$$

For the undamped system, if $M$ and $K$ are non-singular, as they are for the modeling of MEMS in this thesis, all odd moments $m_{1}, m_{3} \ldots, m_{n}$ are zero.

This is most simply shown by looking at the moments in more detail as 


$$
\begin{aligned}
& m_{0}=K^{-1} F \\
& m_{1}=\left(K^{-1} D\right) m_{0} \\
& m_{2}=\left(K^{-1} M\right) m_{0}-\left(K^{-1} D\right) m_{1} \\
& m_{3}=\left(K^{-1} M\right) m_{1}-\left(K^{-1} D\right) m_{2} \\
& m_{4}=\left(K^{-1} M\right) m_{2}-\left(K^{-1} D\right) m_{3} \\
& \vdots \\
& m_{n}=\left(K^{-1} M\right) m_{n-2}-\left(K^{-1} D\right) m_{n-1}
\end{aligned}
$$

If the system is undamped, $D=0$, then it is clearly seen that

$$
\begin{aligned}
& m_{0}=K^{-1} F \\
& m_{1}=\left(K^{-1} D\right) m_{0}=0 \\
& m_{2}=\left(K^{-1} M\right) m_{0} \\
& m_{3}=\left(K^{-1} M\right) m_{1}-\left(K^{-1} D\right) m_{2}=0 \\
& m_{4}=\left(K^{-1} M\right) m_{2}-\left(K^{-1} D\right) m_{3}=\left(K^{-1} M\right) m_{2} \\
& \vdots \\
& m_{n}=\left(K^{-1} M\right) m_{n-2}-\left(K^{-1} D\right) m_{n-1}=\left(K^{-1} M\right) m_{n-2}
\end{aligned}
$$

This is also shown in equation 7.2.6.

\section{Summary of Results of Moment Analysis: Analytical and Numerical}

Moments of varying sized systems were examined in detail, with the following observations:

1. The moments of a system at zero damping resulted in equal moments for every odd moments and all zero moments for even numbered moments.

2. When damping was introduced into the system, the even moments became non-zero. Their values were scaled by a factor the damping was scaled to, but whose magnitude were on the order of $10^{-20}$ times lower than that of the odd moments. 
3. Once the system became critically damped or overdamped, odd moments $\geq 3$ exhibited a significant decrease in magnitude.

4. The sum of the moments at zero damping resulted in eigenvalues/poles that were zero.

5. In smaller models the numerical values of the moments were exactly equal, but as the size of the models increased slight numerical differences were observed, suggesting numerical round off errors in generating larger FEM models.

\subsubsection{The Effect of Damping on the orthogonal $Q$-matrix}

The effect of varying the damping on the formation of the orthogonal $Q$-matrix in the Arnoldi algorithm was investigated and yielded some interesting observations:

1. The 16 damped companion forms generated orthogonal matrices that varied significantly from each other, while the two that passed the stability tests for the Arnoldi algorithm were almost identical.

2. The rank of orthogonal $Q$-matrices, concatenated vertically to determine any linear dependency, showed that for a matrix of size $N \times n$ (where $n$ is the number of moments, and $N$ is the size of the original system), the rank of the 2 combined matrices stayed at $N$, regardless of the damping factor. This held true for smaller models but when the models became larger, the rank was not exactly $N$ when using the Arnoldi algorithm. For example for a large model with 20 moments, the concatenated orthogonal matrices will have a rank of 30 when using Arnoldi. However, if the numerical tolerance was decreased, the rank approached 20 as shown in Figure 7.1. This actually suggested that there was more 
numerical error when performing model reduction using the Arnoldi algorithm. When using SOAR, the rank always stayed at $N$ when using the SOAR algorithm for any size of model. From these observations, it can be concluded that the inclusion of damping in the original system matrices simply scales the generated orthogonalized matrix, $Q$, if no numerical error is present.

3. When damping was included, there was loss in orthogonality between the eigenvectors that form the $Q$-matrix, however, the eigenvectors were perfectly orthogonal when formed with no damping.

4. The orthogonal matrix, $Q$, could be generated from any of the 16 companion undamped forms, and used to then transform the full system, now formulated to include damping, in any of the 16 forms to the reduced model, with low relative errors and a good match to the full response.

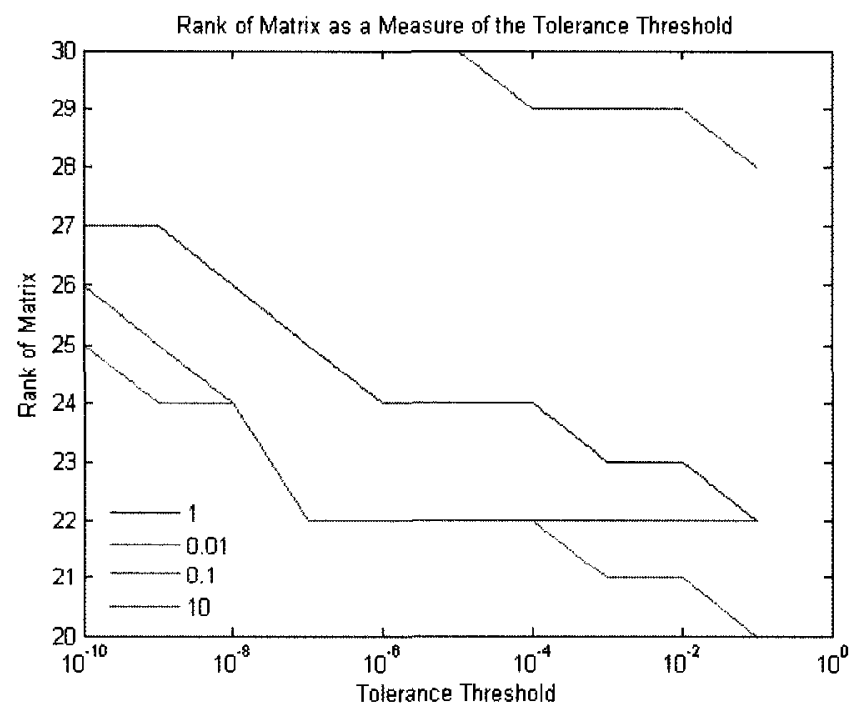

Figure 7.1: Rank as a Measure of Tolerance Threshold 


\subsubsection{Effect of Damping on the Stability of Arnoldi and SOAR}

Analytically, it was seen that the effect of damping on a system was numerically negligible on even-numbered moments, while the contribution to odd-numbered moments was on the order of $<<10^{-18}$ lower than the magnitude of the even-numbered moments. This small contribution would suggest that damping should not play an important role in the formation of the reduced model. However, it was clearly demonstrated in the examination of the stability of Krylov subspace methods for model reduction on second-order systems in Chapter 4 , and the consequent comparison of the two types of the model reduction techniques, Arnoldi and SOAR in Chapter 5 and Chapter 6, that the Arnoldi algorithm became unstable and required higher number of moments to achieve a stable result as the system approached critical damping. It was also evident that a higher number of moments could not guarantee a stable response.

Additionally, as previously noted, the companion form for the second-order differential equation can be formed with sixteen different configurations. Of these sixteen, only two passed the tests to show if a system was stable and passive. However, for these two configurations examined in Chapter 6, it was found that the stability of the response in the time domain became heavily dependent on the damping of the system.

As the effective implementation of model reduction is to significantly reduce the size of a model to minimize computational effort, using a large number of moments defeats this purpose. It is also important to note that the SOAR implementation of model reduction exhibits none of these flaws, except when the modeling technique based on SUGAR was used. In this case, it was the ill-conditioning of the resulting global systems matrices for larger models that brought about the instability of SOAR. 
The growing instability of reduced models implemented using the Arnoldi algorithm for 'stable' companion forms, is shown in Figure 7.2, where any pole on the right-hand side of the complex plane indicates an instability in the system. Stable systems have poles that only lie on the left-hand side of the of the complex plane, or have poles that cluster very close to zero, or even lying on the real-axis.

Discussion It was clearly seen that as the damping increased, the poles of the system moved to the the unstable right-hand side of the positive real axis. As the damping approached zero, the poles clustered around the imaginary axis, but any poles lying in the right-hand side of the positive real axis were so small in magnitude and so close to the axis, their contribution became inconsequential and did not cause any instability of the system. 

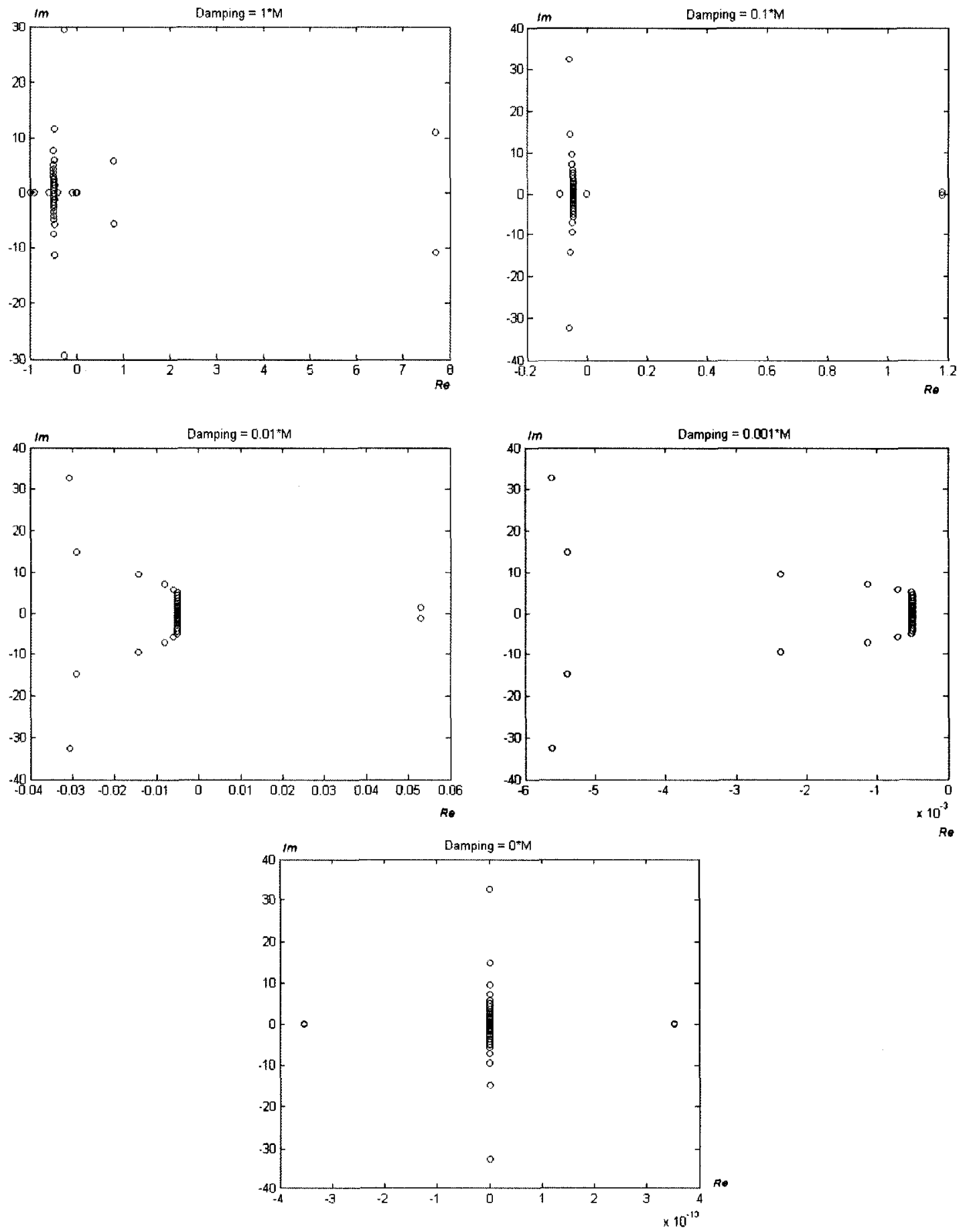

Figure 7.2: Eigenvalues of Arnoldi Reduced Models with a Range in Damping, 540-node reduced model with 30 moments 
For the same 540-node model, now implemented using the SOAR model reduction technique, the stability of the system is shown in Figure 7.3. 

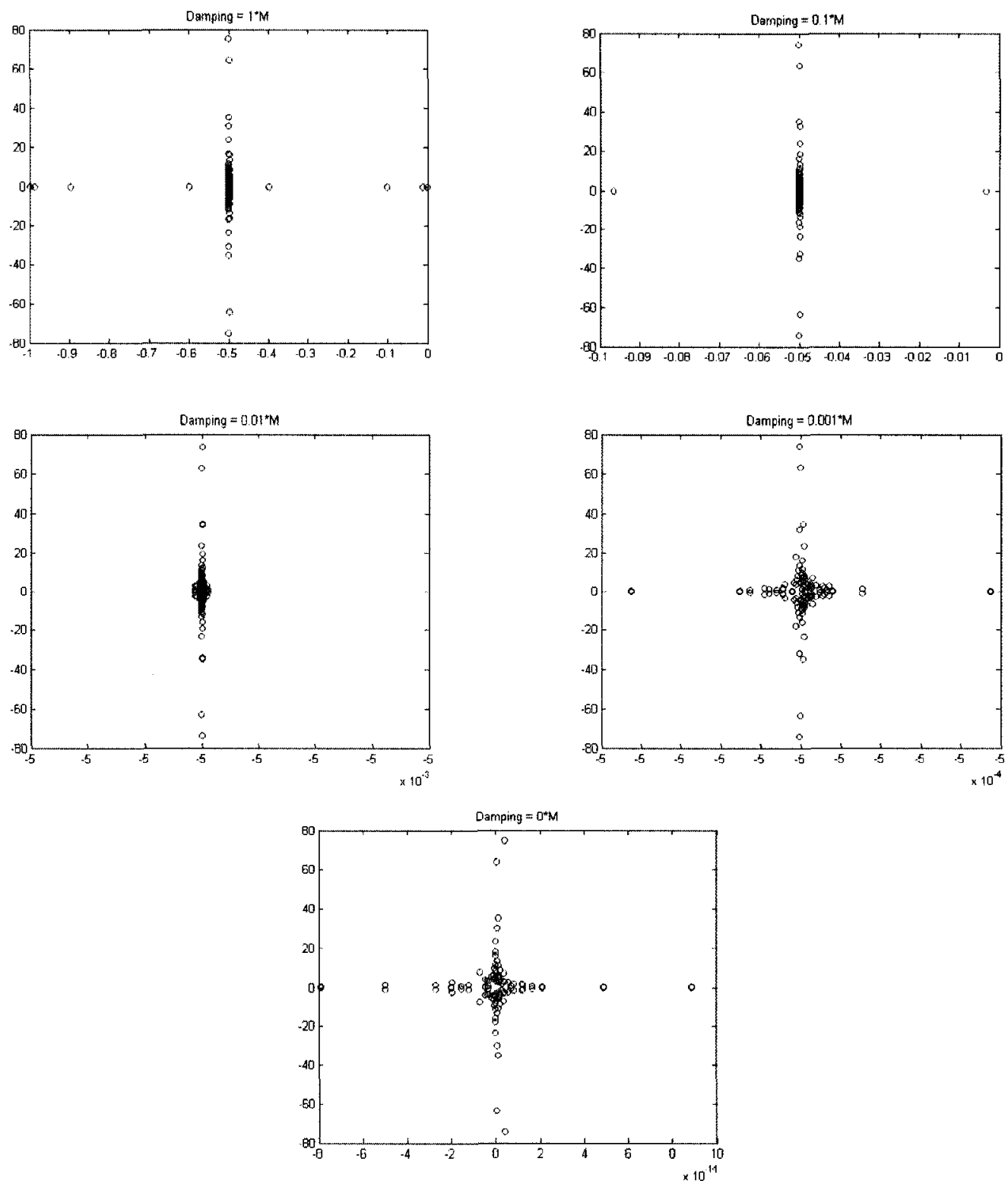

Figure 7.3: Eigenvalues of SOAR Reduced Models with a Range in Damping, 540-node reduced model with 30 moments 
Discussion In Figure 7.3, the reduced model generated using the SOAR procedure illustrated pole clustering that was predominantly in the left-hand plane. It was also observed that, although some of the poles were on the unstable right side of the real axis as the damping approaches zero, the magnitude of the unstable poles were such that the system stays stable and those poles were few in comparison to the much larger number of stable poles.

\subsubsection{Summary on the Effects of Damping on the Stability of Reduced Models}

The analytical analysis of the moments of a system with and without damping shows that damping only affected even moments which were zero when there was no damping in the system. The numerical analysis indicated that the contribution of damping to the even moments was very small in magnitude compared to the odd moments, and was scaled to the damping factor. The low order of magnitude of this contribution suggested that the overall effect of damping on the moment analysis of the system should be negligible and that damping can be ignored in the numerical analysis of the system and, hence, the implementation of model reduction techniques. However, in the analysis of the eigenvalues of the 540-node reduced model, it was clearly seen that as the damping increased when model reduction was implemented using the Arnoldi algorithm, the system became more unstable with poles lying in the unstable right-hand side of the complex plane. SOAR remained marginally stable, with all poles clustering around the real axis with magnitudes close to zero, as did the Arnoldi algorithm with a damping of zero.

As noted from the above observations, damping introduced only a very small numerical 
contribution to the even moments of a system, suggesting that it could be ignored in the implementation of model reduction techniques and the formation of the orthogonal $Q$-matrix. As such, a projection formulation of the undamped orthogonal $Q$-matrix will now be considered in creating damped reduced models.

\subsection{Undamped Krylov Subspace Methods and Second-Order Systems}

On further investigation of the effect of damping on the Arnoldi algorithm, an interesting experimental observation was made. In forming the companion form for the Arnoldi algorithm with the damping of the system set to zero the resultant orthogonal matrix, $Q$, could be used to generate the reduced model of the full system using any level of damping with an acceptably low relative error, even if the damping was much larger than the critical damping.

It was also observed that if the damping was set to zero for any of the sixteen companion form configurations used the eigenvalues of the full system and, therefore, the initial solution used to build the orthogonal space in the Arnoldi algorithm were always the same. This implied that the initial choice of any of the sixteen companion forms became irrelevant, as the undamped companion forms passed the stability tests.

Finally, it was also seen that any of the sixteen companion forms could be used to build the reduced model, regardless of the initial form used to build the $Q$ matrix. This was illustrated in Figure 7.4 where the companion form used to implement the Arnoldi algorithm was randomly chosen from the available 16 and the generated $Q$-matrix was then used to form the reduced model using any of the 16 companion forms (only 8 are shown for easier illustration), using the 
congruent transformation discussed in Chapter 3.

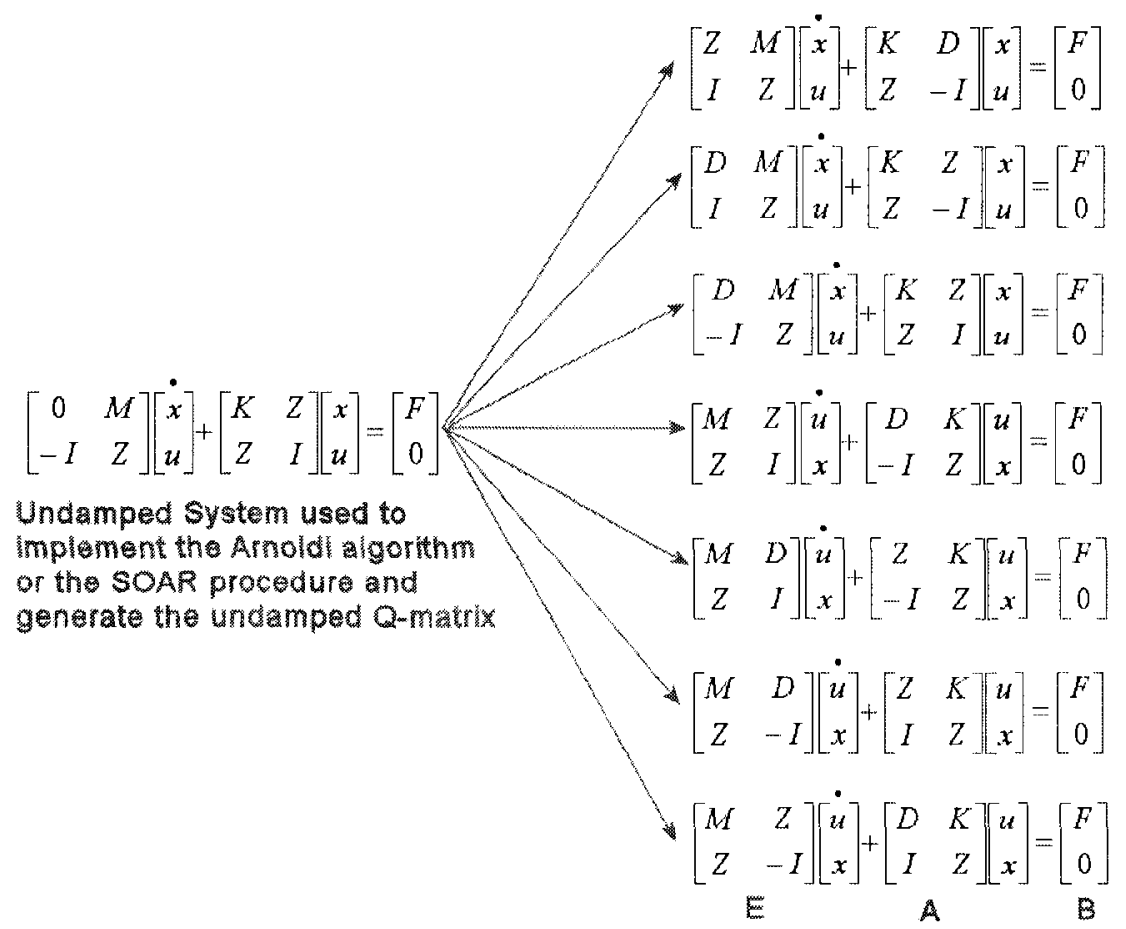

First 8 companion forms the undamped Q-matrix is mapped to form reduced models, as shown below

$$
\begin{aligned}
& E_{\text {red }}=Q^{T} E Q \\
& A_{\text {red }}=Q^{T} A Q \\
& B_{\text {red }}=Q^{T} B
\end{aligned}
$$

Figure 7.4: Relative Errors of Reduced Models using Rayleigh Damping

The literature, [18], [60], [61], [62], [63], and [64] states that the damping matrix does not play a significant role in generating the orthogonalized Krylov subspace for a low-dimensional basis, $Q$, as it is assumed that the most essential information of the system is contained in the mass, $M$, and stiffness, $K$ matrix. However, this observation has not been explicitly mathematically proven and is stated as being based on engineering intuition. 
As noted in the previous section, it was observed that the effect of damping on the computation of the moments of a system was numerically negligible on even-numbered moments, and it did not affect the numerical magnitude of odd-numbered moments. This observation reaffirmed that made in the literature, but efforts to provide a solid mathematical proof was impossible. The only reasonable explanation stems from the fact that, in structural dynamics, the damping of the system is a linear combination of the mass, $M$ and the stiffness, $K$, matrices. This is well known as Rayleigh Damping and is defined as $D=\alpha M+\beta K$. In the FEM and SUGAR models created for this thesis, the damping is set to $D=\alpha M$. The relation of the damping of the system to the mass and stiffness matrix, and the observations of the numerical contributions of damping to the moments of the system suggested that damped moments might be a perturbation of the undamped moments. However, efforts to validate this through matrix perturbation theory was beyond the scope of this thesis.

\subsubsection{The Undamped SOAR Procedure}

The observation that reduced models constructed using systems initially set with zero damping and then remapped to the original model with added damping resulted in an excellent match to the response of the full model with the SOAR procedure.

\subsubsection{The Undamped Arnoldi Algorithm}

As previously discussed, the Arnoldi algorithm requires the second-order system to be converted into first order by using a companion form. Second order systems can be written in sixteen different companion forms, of which it was demonstrated that only two passed the tests 
to preserve the stability of the system. The second-order undamped system simplified to

$$
\begin{aligned}
& M \ddot{u}(t)+K u(t)=F b(t) \\
& w=L^{T} u(t)
\end{aligned}
$$

By eliminating the damping matrix, the number of companion forms was reduced from sixteen to eight, and consequent tests for stability on the resulting matrices showed that the undamped companion form passed. Also, the initial solution used in the Arnoldi algorithm became constant for all the 16 forms.

In the numerical analysis presented below, the undamped $Q$-matrix formed in the implementation of the Arnoldi algorithm and used to generate the reduced damped models gave excellent matching to the full response, similar to the SOAR procedure.

\subsubsection{Numerical Analysis of Undamped Krylov Subspace Methods}

In an attempt to conclusively demonstrate that the orthogonal Krylov subspace generated with an initially undamped system could then be used to create reduced models with damping included, a cantilever of 1350-nodes was modeled using two types of damping, Rayleigh damped, $D=\alpha M+\beta K$, and the damping used for FEM modeling previously, $D=\alpha M$. The focus was on implementing the Arnoldi algorithm, as this type of model reduction has been shown to be far more unstable than suggested in the literature and, as will be demonstrated in the following results, the effect of varying damping using Rayleigh damping or FEM damping, to generate reduced models using an orthogonal $Q$-matrix with the damping set to zero, proved to be inconsequential.

The aim of taking this approach was to conclusively verify that the accuracy of the results 
was not dependent on the fact that in FEM modeling the damping is a scaled quantity of the mass matrix, $M$, and that it could be set to any value. It was used as a method of showing that, even if the damping was set so the system became highly overdamped, the resulting reduced model was still stable.

For the 1350-node model, each of the sixteen companion forms was used to construct a zerodamped orthogonal $Q$-matrix. Following this, the reduced models of all sixteen companion forms were built with the damping set to $D=\alpha M$, with values of $\alpha$ being set to $0.001,0.01$, $0.1,1$, and 10. In total, this gave 1280 sets of results for comparison to the full models set to the same damping.

It could be clearly seen that, as shown when modeling more complex structures, any of the sixteen companion forms could be used to form the zero-damped orthogonal $Q$-matrix. This in turn could then generate the reduced models and still achieve an excellent match to the full system. The low relative errors obtained are shown in Figure 7.8

The models were then damped using Rayleigh damping, $D=\alpha M+\beta K$. For both the zero-damped $Q$-matrix and the reduced model, only one companion form of the sixteen was arbitrarily chosen, and the values for $\alpha$ and $\beta$ were both varied from $0,0.001,0.01,0.1,1$, and 10.

\section{Stability Results}

The stability of the systems was also verified by showing the poles of the reduced models in Figures 7.5 and 7.6. Additional eigenvalue analysis of this system is provided in Appendix E. 

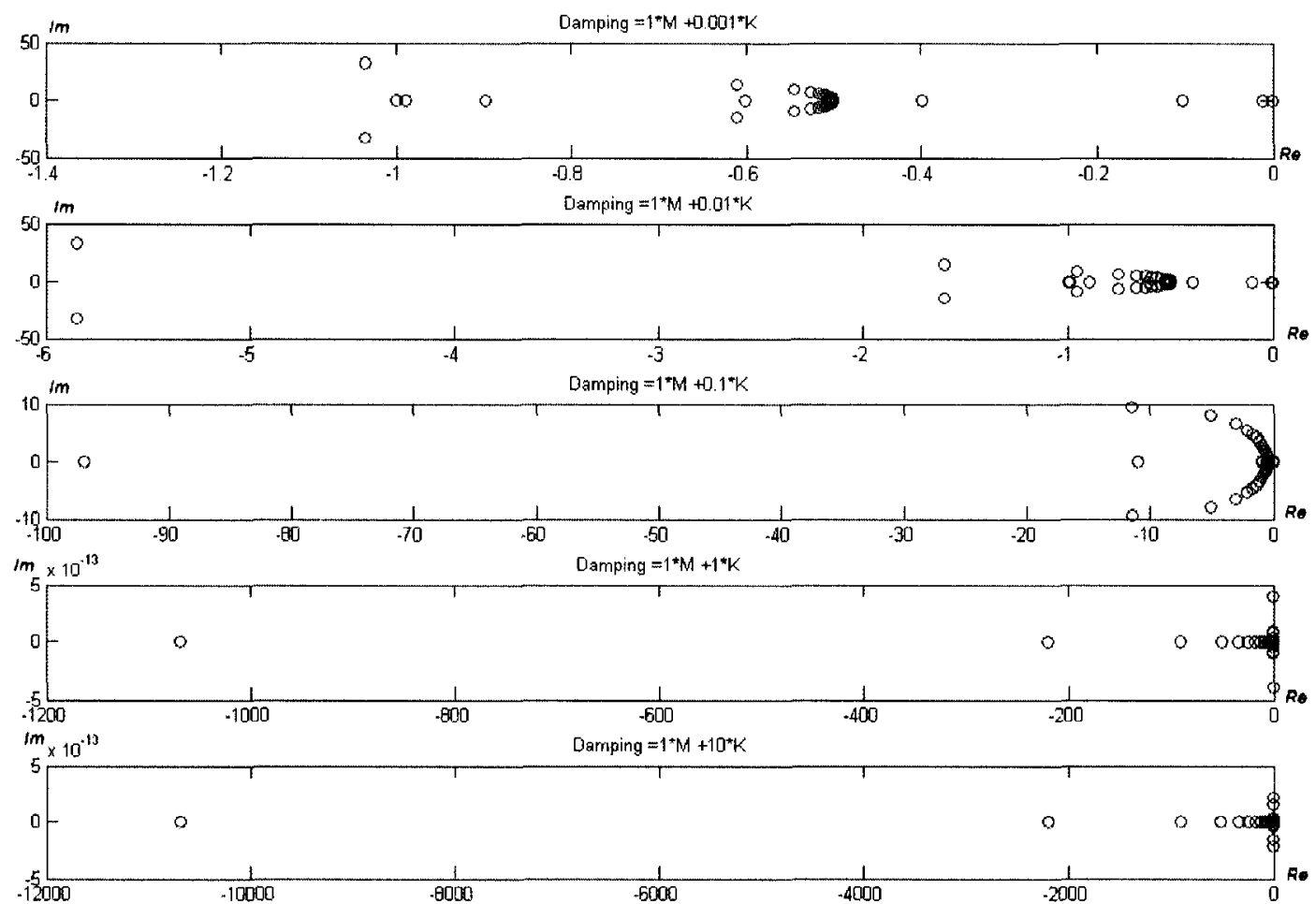

Figure 7.5: Eigenvalues of Reduced Models using Rayleigh Damping, $\alpha=1$ 

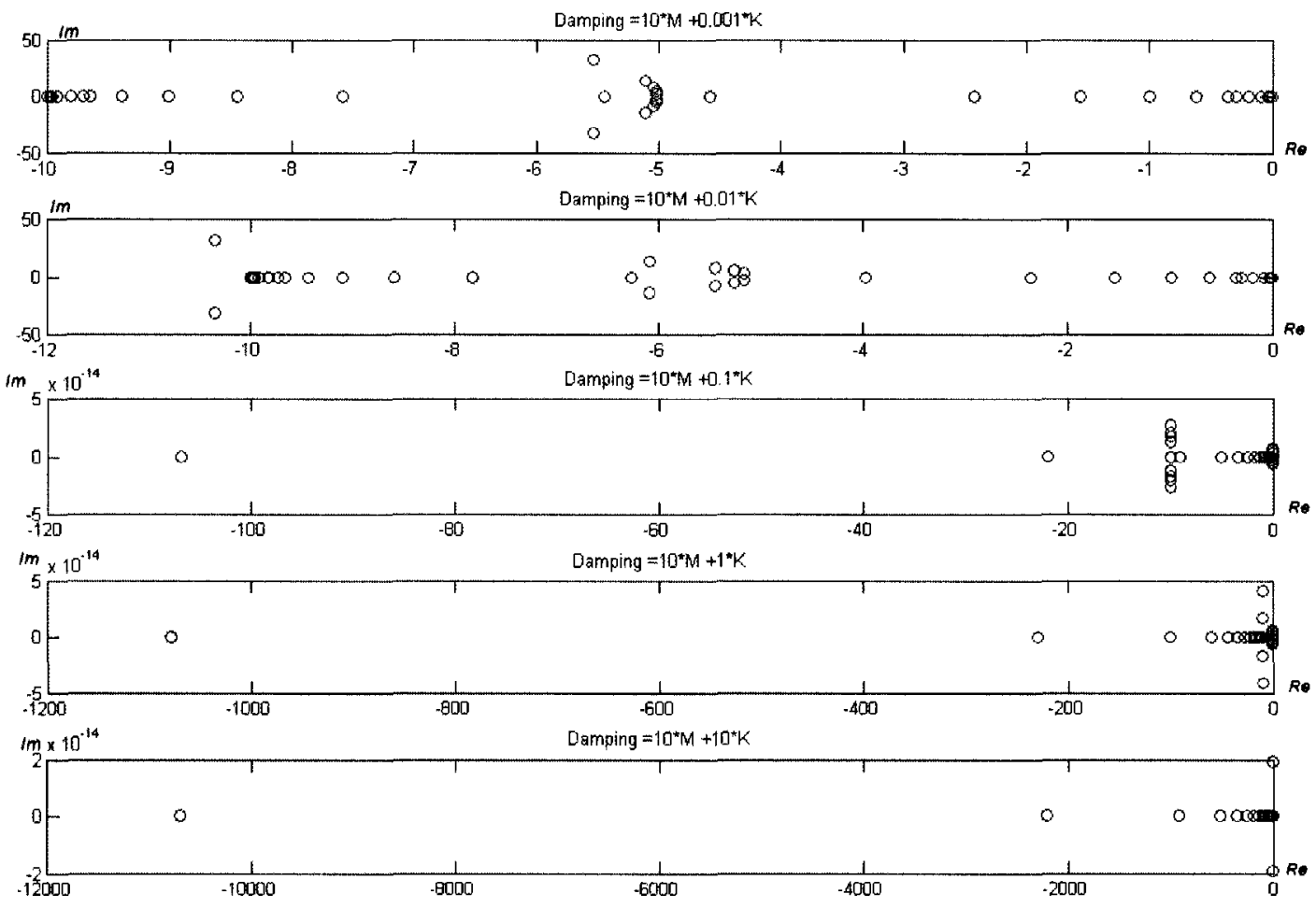

Figure 7.6: Eigenvalues of Reduced Models using Rayleigh Damping, $\alpha=10$ 
Discussion: The reduced models were clearly stable as the poles of the system were all on the left-hand side of the imaginary axis regardless of the damping, and no poles were found in the right-hand side of the complex plane which is an indicator of instability.

\subsubsection{Time Domain Analysis}

The time domain responses of the full model and the reduced model for selected Rayleigh damping will be considered for the 1350-node cantilever modeled in FEM. As shown in Figure 7.8 , the relative errors for the time domain solution for the 1350-node cantilever was very low for all variable Rayleigh damping that the model was formed with. 

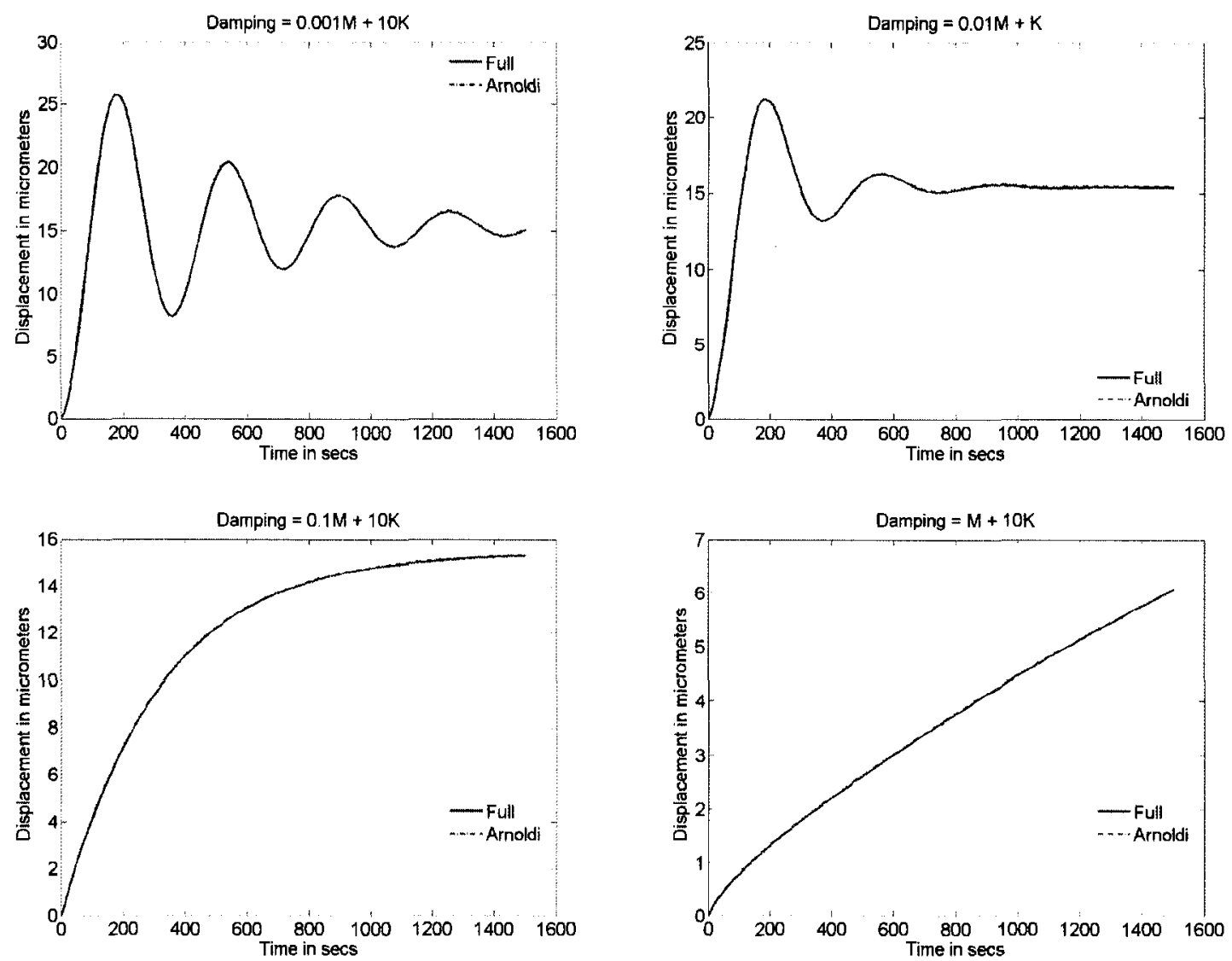

Figure 7.7: Time domain Response Comparison of Reduced Models and Full model using Rayleigh Damping 

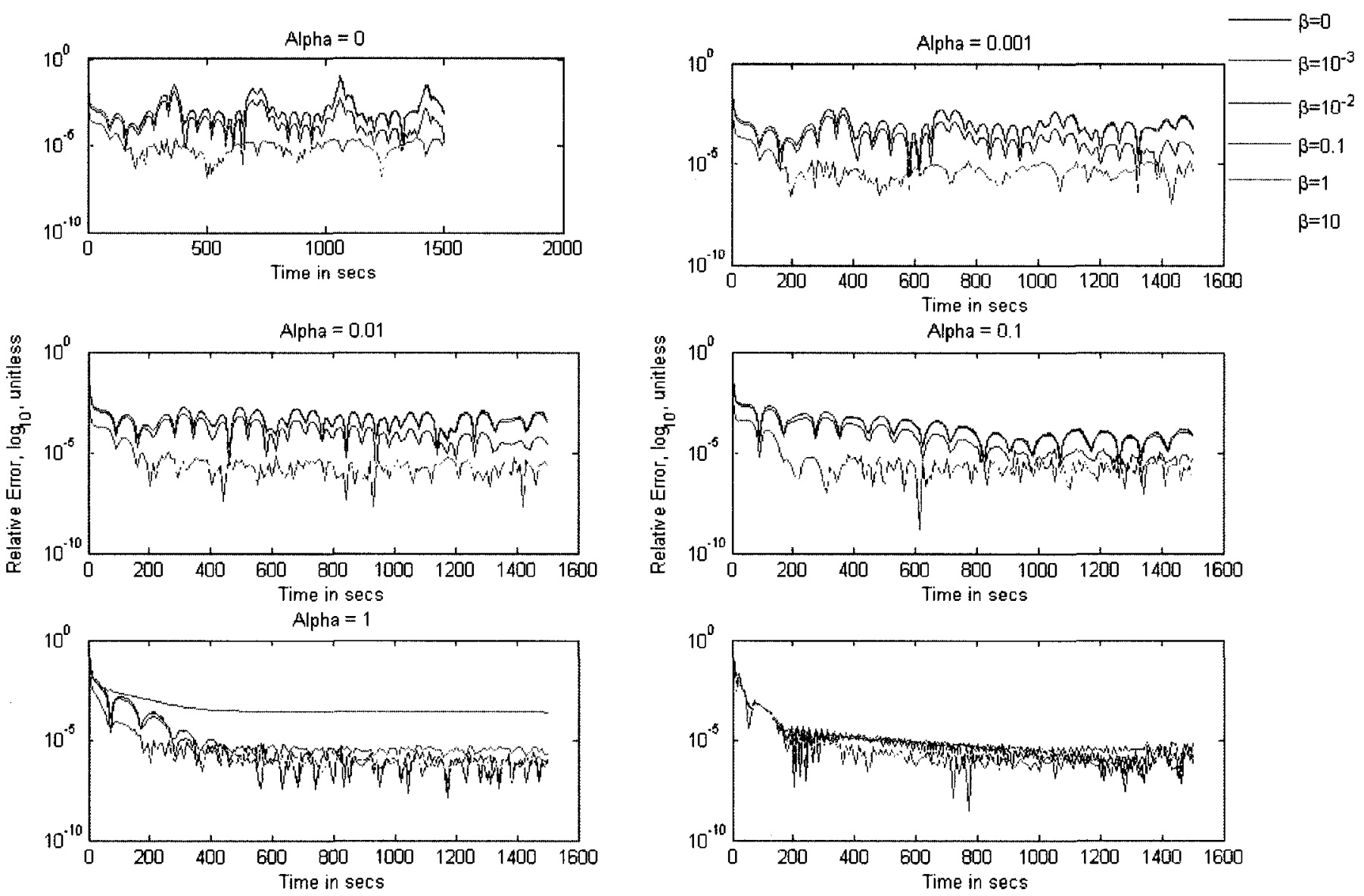

Figure 7.8: Relative Errors of the TD Simulation for Reduced Models using Rayleigh Damping

Discussion: From Figure 7.7, the reduced models provided an excellent match to the full models in the time domain. However, the cantilever model is arguably a very simple structure to model, so to substantiate the robustness of performing model reduction using this observation, more complex, real-world structures used in many existing engineering applications will be considered. 


\subsubsection{Numerical Analysis of Complex Structures}

The procedure outlined in the preceding section was then applied to more complex structures to verify that this method of constructing the reduced models was accurate. This was important as the simplicity and symmetry of the cantilever could result in favorable results, so real structures that exhibit greater complexity, some asymmetry and also used in real MEMS applications were designed using the Atar FEM modeling tool. The model reduction technique will focus on the Arnoldi algorithm again.

\subsubsection{Serpentine}

This basic serpentine structure shown in Figure 7.9 is commonly used in many MEMS applications such as actuators and micromirrors. Its spring-like structure provides a high level of sensitivity that is especially useful for low resonant frequency applications.

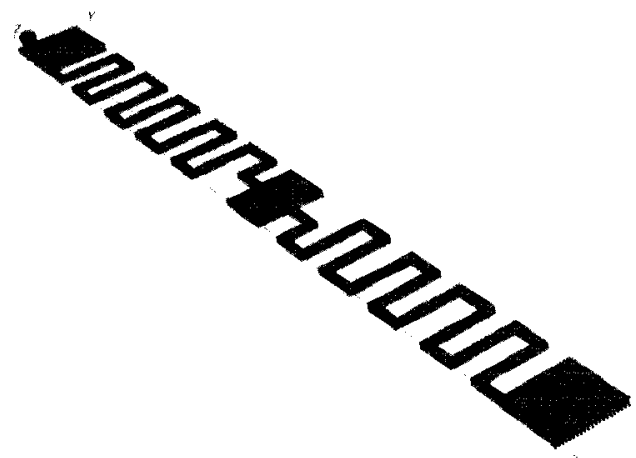

(a) $\mathrm{a}$

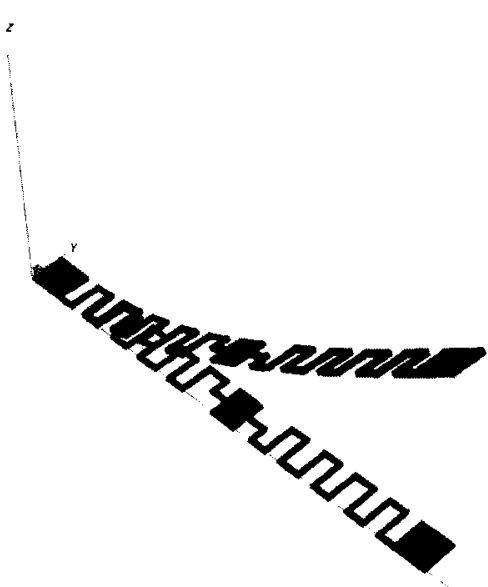

(b) $\mathbf{b}$

Figure 7.9: Serpentine structure used to verify $Q$ formed with $D=0$ 


\section{Response of the Serpentine Structure}

The time domain analysis of the serpentine structure is shown in Figures 7.10 to 7.12 for models that are undamped to overdamped. The lack of symmetry of the structure increases the complexity of the resultant system matrices, which makes it a good analytical tool for comparing the performance of the undamped $Q$-matrix used to generate reduced models.

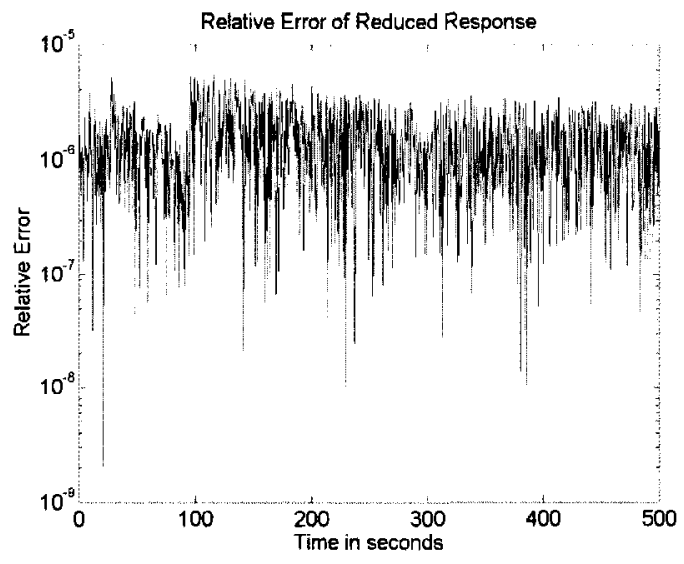

(a) Relative Error of Reduced Model

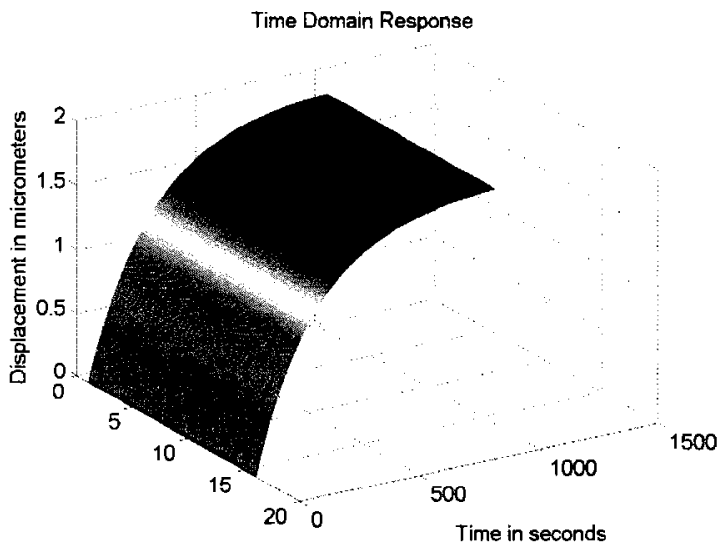

(b) TD Response for 16 companion forms

Figure 7.10: Serpentine structure used to verify $Q$ formed with $D=0$ - Overdamped

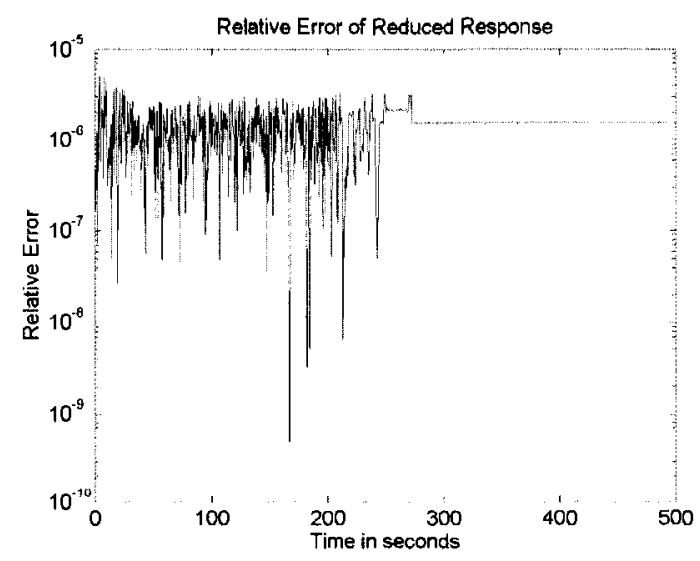

(a) Relative Error of Reduced Model

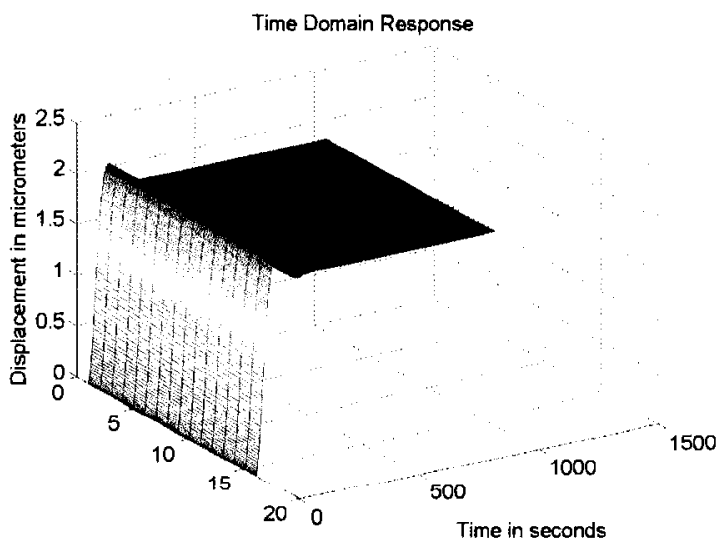

(b) TD Response for 16 companion forms

Figure 7.11: Serpentine structure used to verify $Q$ formed with $D=0$ - Approaching Critical 


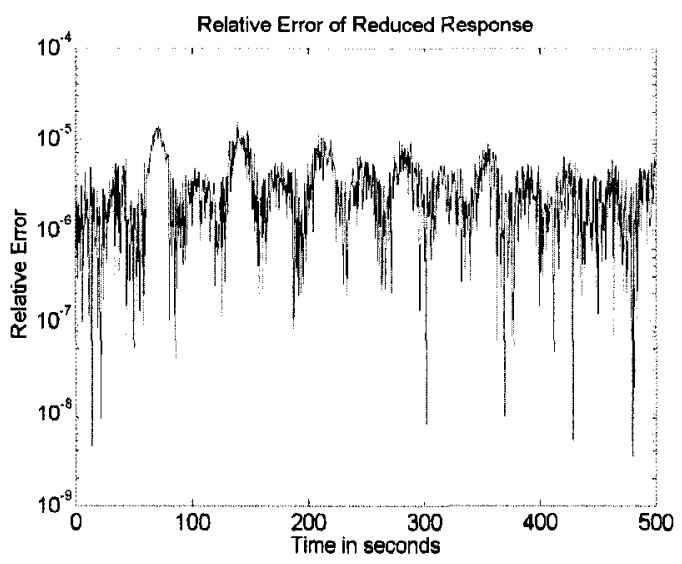

(a) Relative Error of Reduced Model

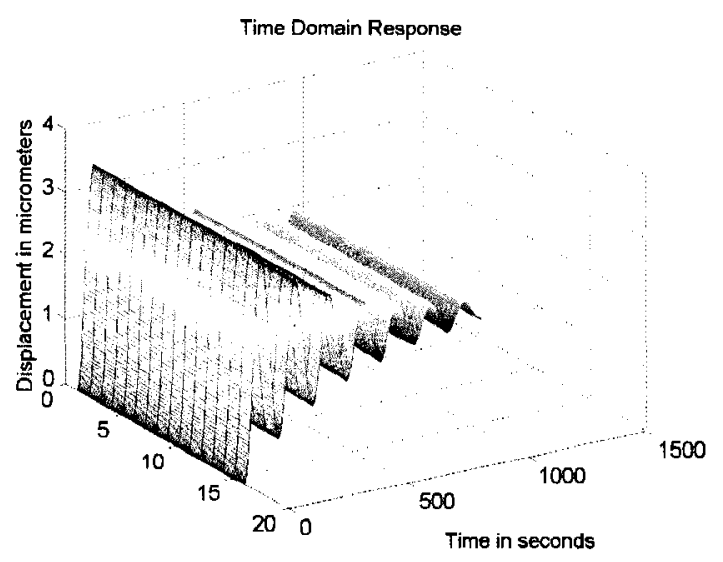

(b) TD Response for 16 companion forms

Figure 7.12: Serpentine structure used to verify $Q$ formed with $\mathrm{D}=0$ - Underdamped 


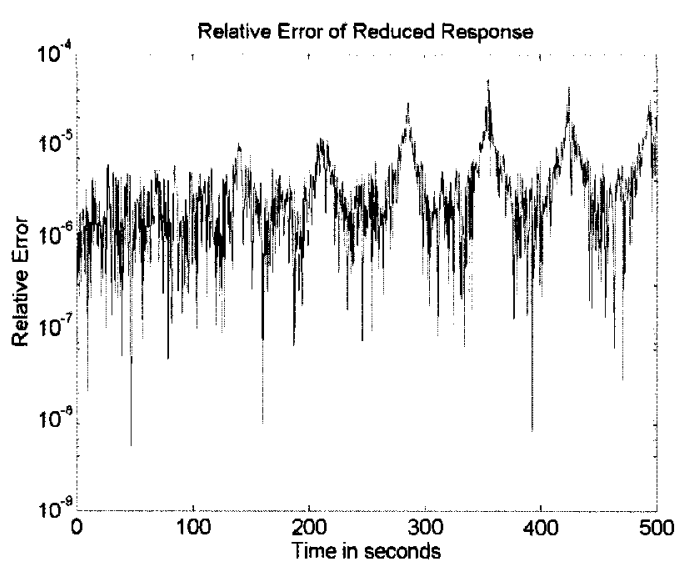

(a) Relative Error of Reduced Model

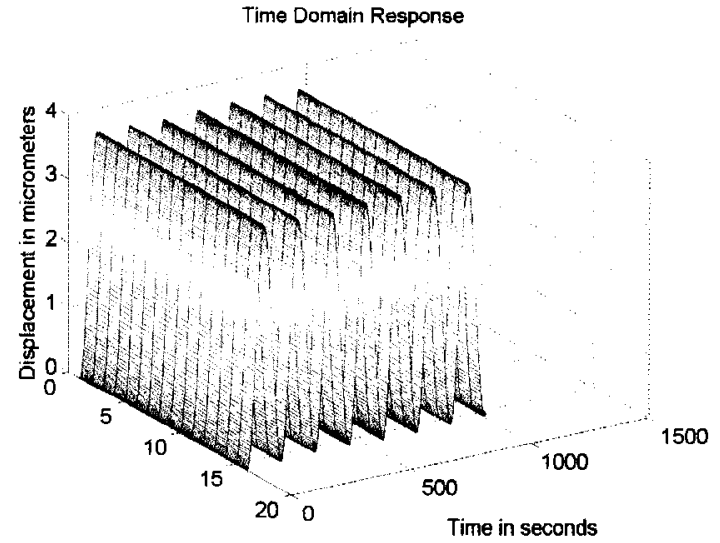

(b) Time Domain Response

Figure 7.13: Serpentine structure used to verify $Q$ formed with $D=0$ - No Damping

Discussion: The time domain analysis shown above demonstrated the robustness and accurate performance of using an undamped system to implement Krylov subspace based model reduction techniques. All 16 companion forms for all levels of damping all matched and, as shown in Figures 7.9(a) to 7.13(a), the relative errors obtained for all levels of damping used to create the reduced serpentine models were very small and on the order of $10^{-6}$. This surpassed some of the previous results shown for the time domain analysis on the Arnoldi algorithm implemented with the companion forms that were shown to be stable.

\subsubsection{H-shape Resonator}

H-shape Resonators, similar to that shown in Figure 7.14, are used in acoustic applications. Although the FEM model is very symmetric and seems simple, mesh refinement at joints and corners make this model more complex than a simple cantilever. 


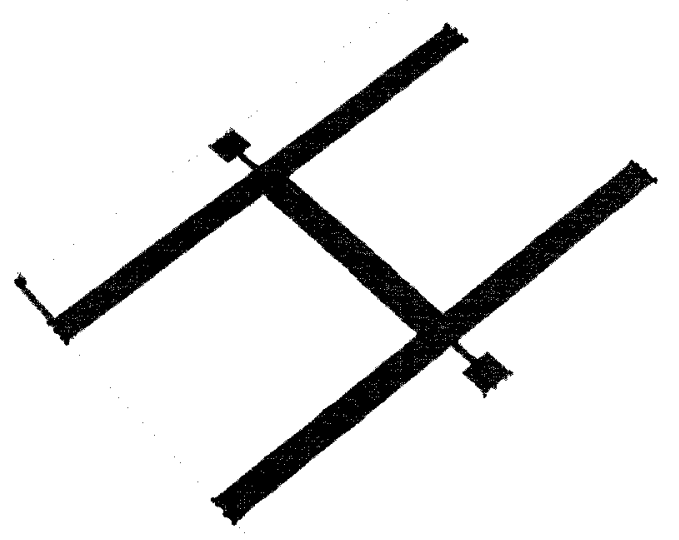

(a) a

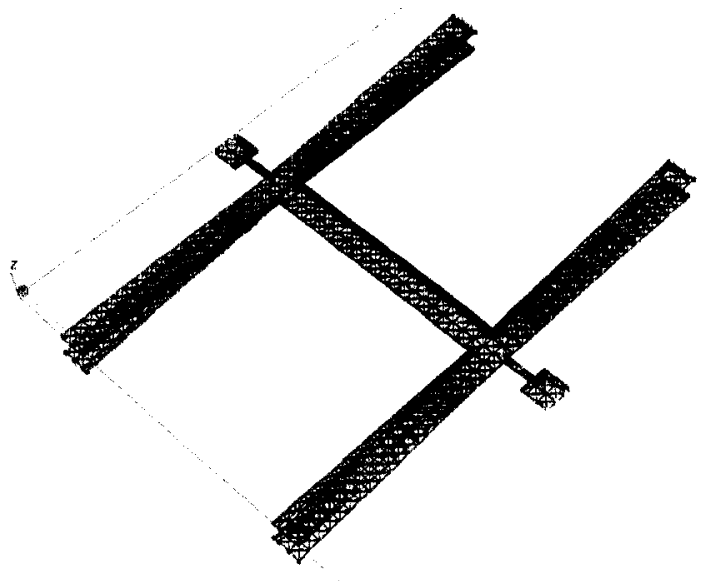

(b) $\mathrm{b}$

Figure 7.14: H-shape Resonator structure used to verify $Q$ formed with $D=0$

\section{Response of the H-Shape Resonator}

Unlike the serpentine structure, the H-shape resonator is a symmetrical structure but can be easily actuated with different upward and downward force at any of the four free ends, making the response of the structure more complex and interesting.

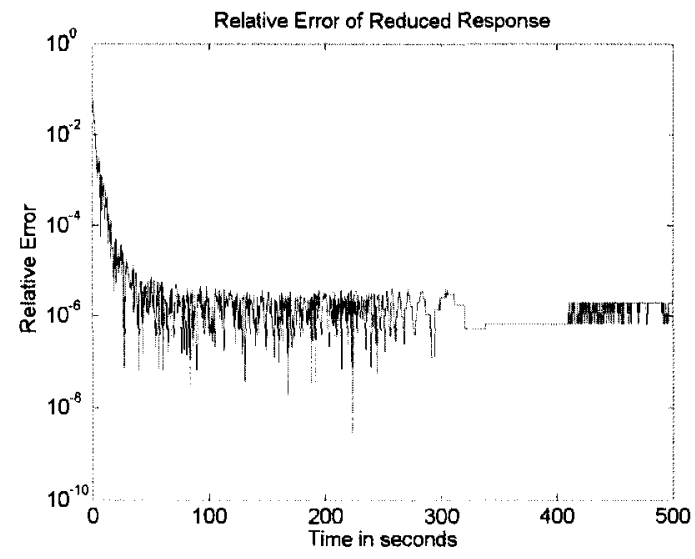

(a) Relative Error of Reduced Model

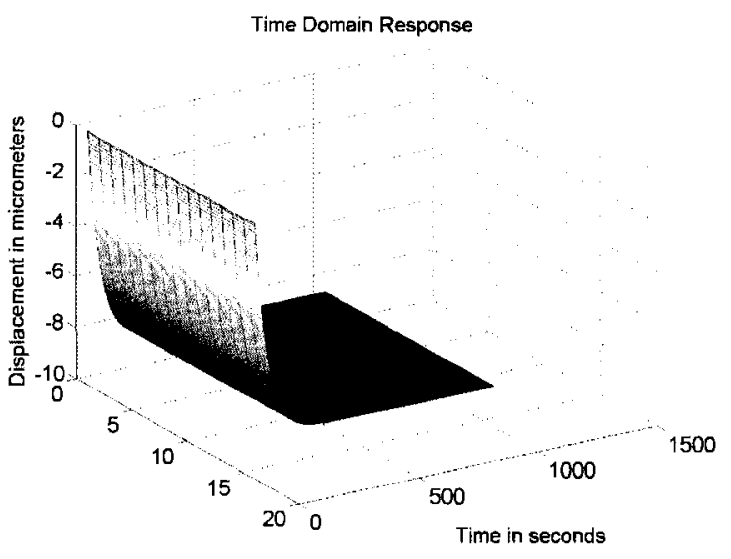

(b) TD Response for 16 companion forms

Figure 7.15: H-shape Resonator used to verify $\mathrm{Q}$ formed with $\mathrm{D}=0$ - Overdamped 


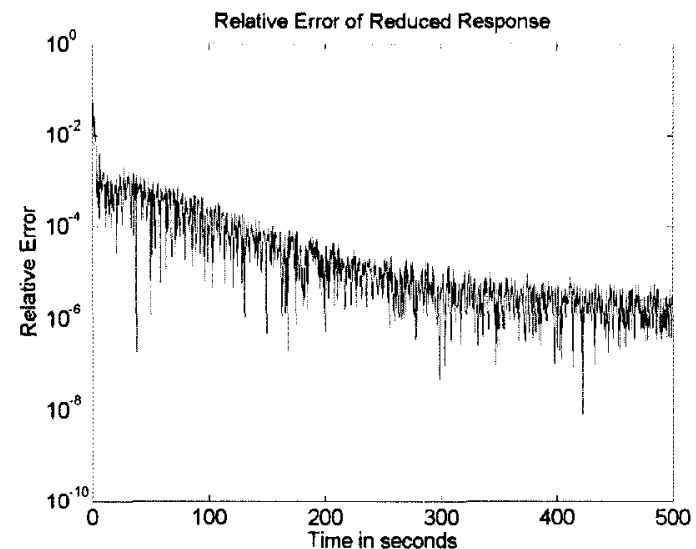

(a) Relative Error of Reduced Model

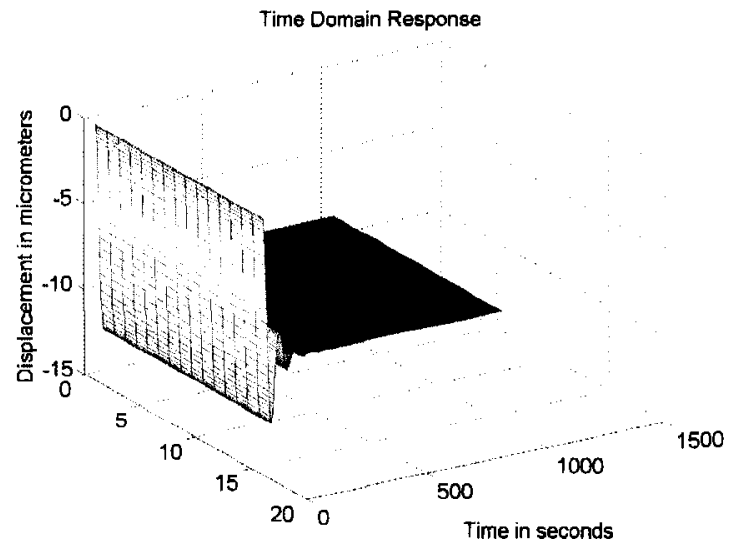

(b) TD Response for 16 companion forms

Figure 7.16: H-shape Resonator used to verify q0 - Approaching Critical

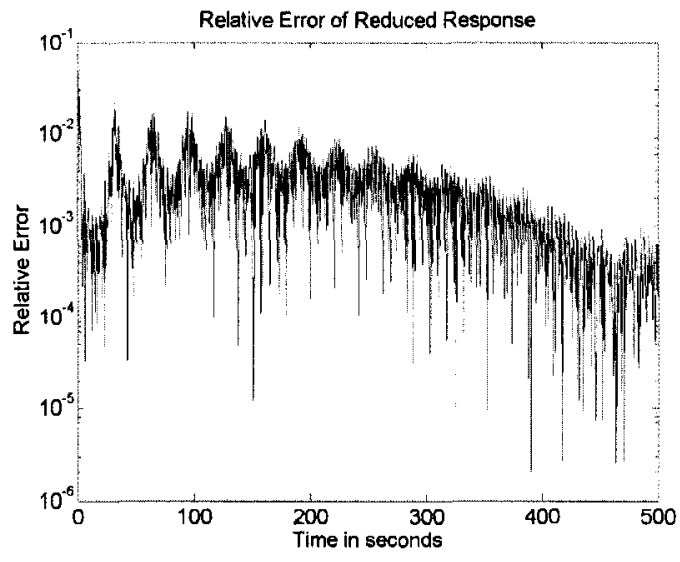

(a) Relative Error of Reduced Model

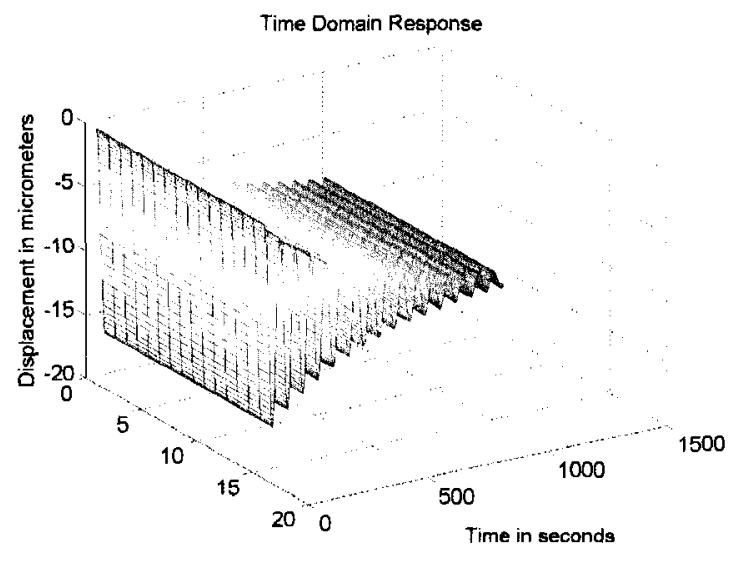

(b) TD Response for 16 companion forms

Figure 7.17: H-shape Resonator used to verify $Q$ formed with $D=0$ - Underdamped 


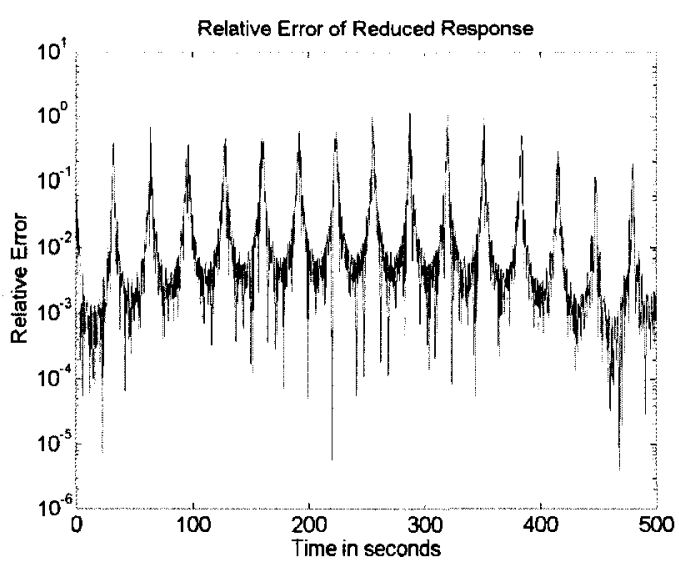

(a) Relative Error of Reduced Model

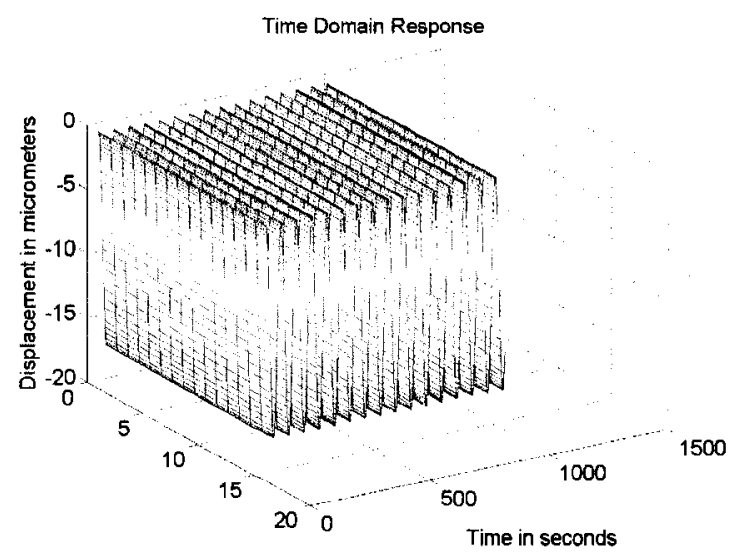

(b) TD Response for 16 companion forms

Figure 7.18: H-shape Resonator used to verify $Q$ formed with $D=0$ - No Damping

Discussion: Like the serpentine structure, the relative errors and time domain response for the H-shape resonator were quite low, as shown in Figures 7.14to 7.18. The time-domain response at any damping was closely matched to the full response as well, which reiterated that generating the orthogonal Krylov subspace with an undamped model was quite suitable for these second-order systems.

\subsubsection{Elevated Inductor}

Elevated Inductors, see Figure 7.19, are used in RFIC applications in passive tuning circuits and as high-impedance chokes for achieving low-voltage operations in advanced silicon IC where processes rely on the negligible DC voltage drop across inductors when utilized as loads or as emitter/source degenerators [65]. The inductor is the most complex structure of the three outlined here and the response of its reduced model will provide a good standard for the robustness of performing model reduction with this technique. 


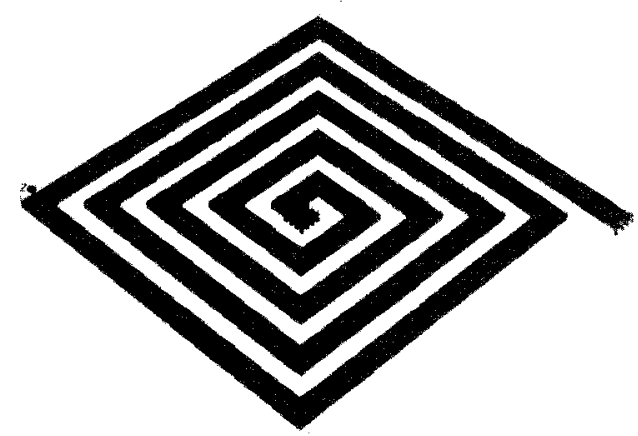

(a) $a$

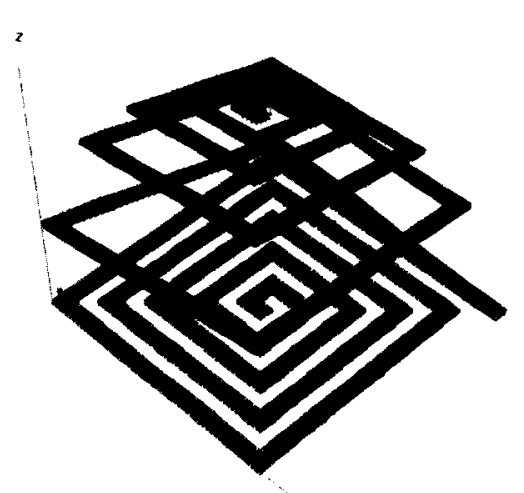

(b) $\mathrm{b}$

Figure 7.19: Elevated Inductor structure used to verify $Q$ formed with $D=0$

\section{Response of the Elevated Inductor}

Similarly to the other two structures discussed above, the time domain response for this structure is analyzed using different amounts of damping ranging from undamped to overdamped. 


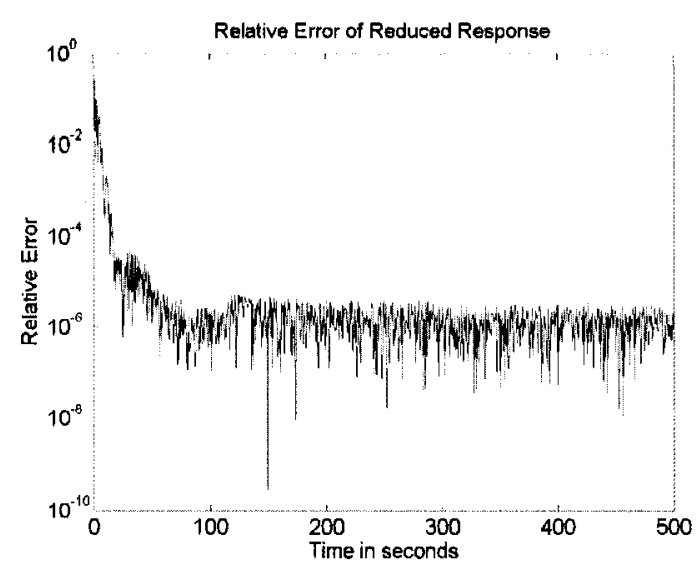

(a) Relative Error of Reduced Model

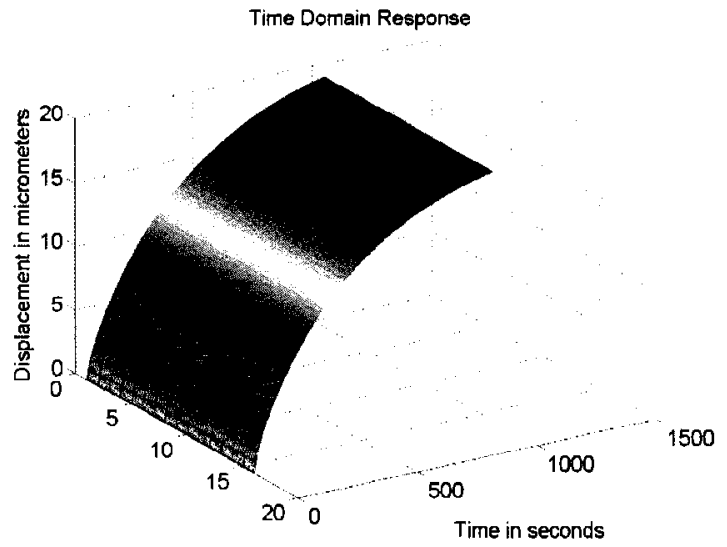

(b) TD Response for 16 companion forms

Figure 7.20: Elevated Inductor used to verify $Q$ formed with $D=0$ - Overdamped

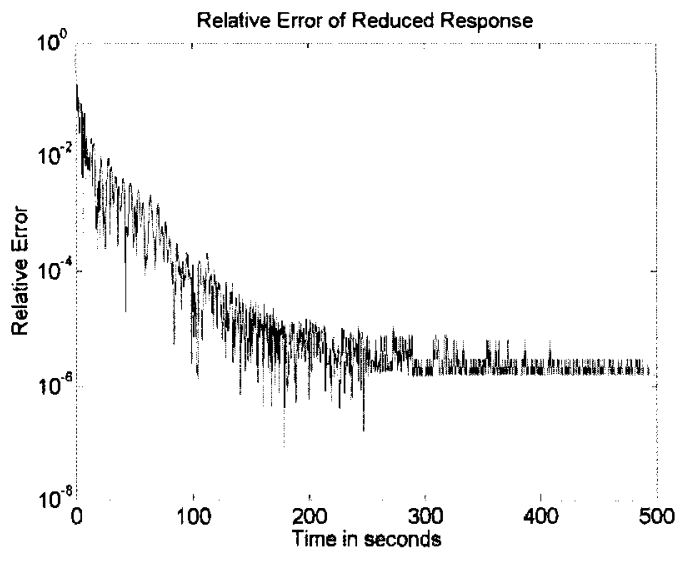

(a) Relative Error of Reduced Model

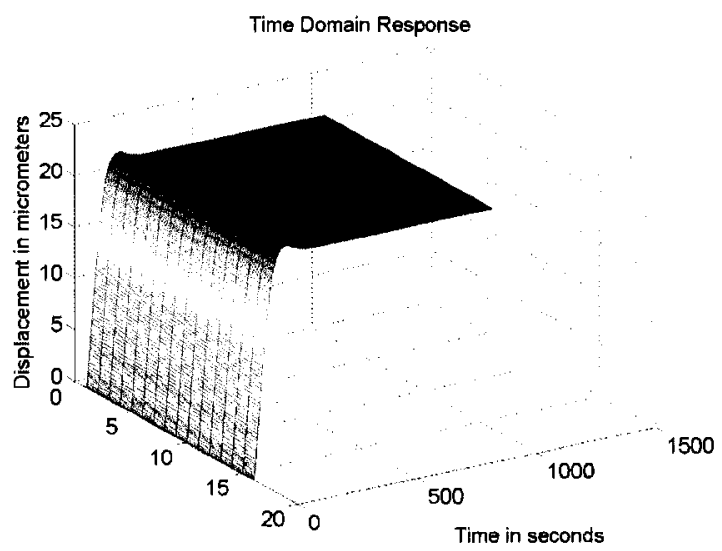

(b) TD Response for 16 companion forms

Figure 7.21: Elevated Inductor used to verify $Q$ formed with $D=0$ - Approaching Critical 


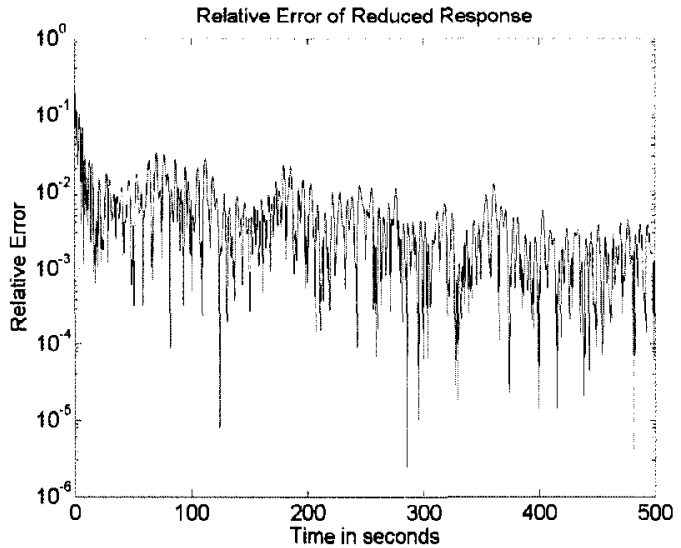

(a) Relative Error of Reduced Model

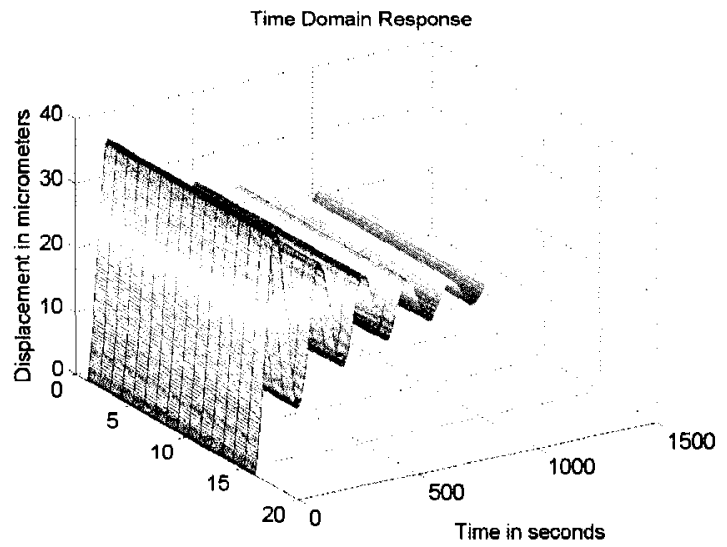

(b) TD Response for 16 companion forms

Figure 7.22: Elevated Inductor used to verify $Q$ formed with $D=0$ - Underdamped

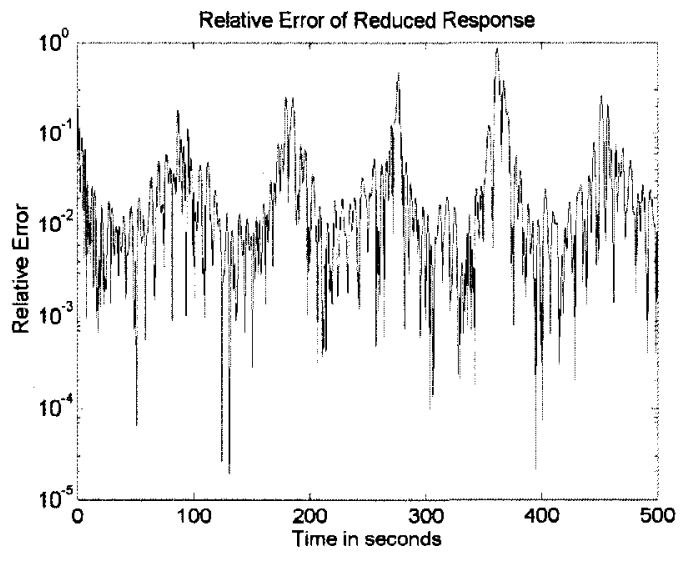

(a) Relative Error of Reduced Model

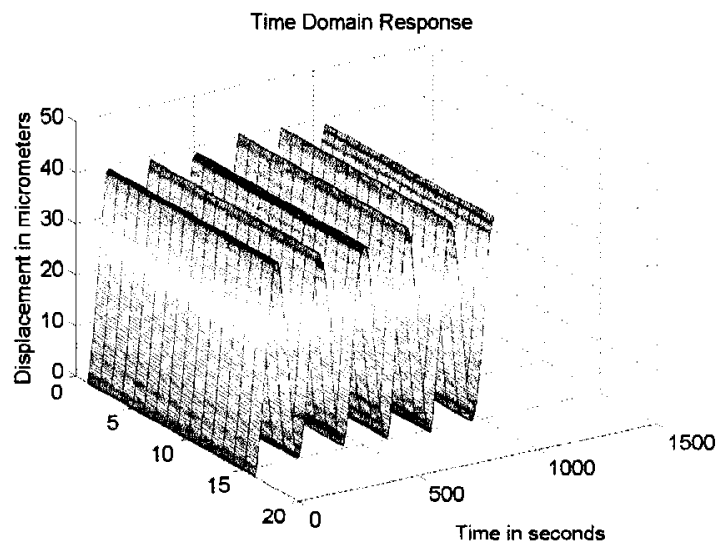

(b) TD Response for 16 companion forms

Figure 7.23: Elevated Inductor used to verify $Q$ formed with $D=0$ - No Damping 
Discussion: As with the other complex structures examined, the relative errors for the elevated inductor was very low, as shown in Figures 7.19(a) to fig:cmplx:a:sub3-5(a). In addition, the time-domain response at any damping was closely matched to the full response as well, see Figures 7.20 (b) to 7.23 (b) for all 16 companion forms that could be used to implement the Arnoldi algorithm.

\subsubsection{SUGAR Models and SOAR}

The 150-node element SUGAR model that was found to be unstable using the SOAR model reduction technique in the time domain analysis when modeled with any significant damping will now be considered.

Figure 7.24 shows the time domain response comparison to the full model achieved using a damping of zero to construct the orthogonal $Q$-matrix in SOAR. The damping was then included when the model was mapped back to the full system to evaluate the response. The model was remapped with a range of damping from $10^{17}$ to $10^{20}$.

It was clearly seen that the match was exceptional. The relative errors presented in Figure 7.25 indicated that the error was well within an acceptable low amount, even lower than model reduction performed with the damping initially included.

Model reduction of the 150 element SUGAR model was then performed using the Arnoldi algorithm and, as could be seen in Figure 7.26, for all 16 companion forms. 

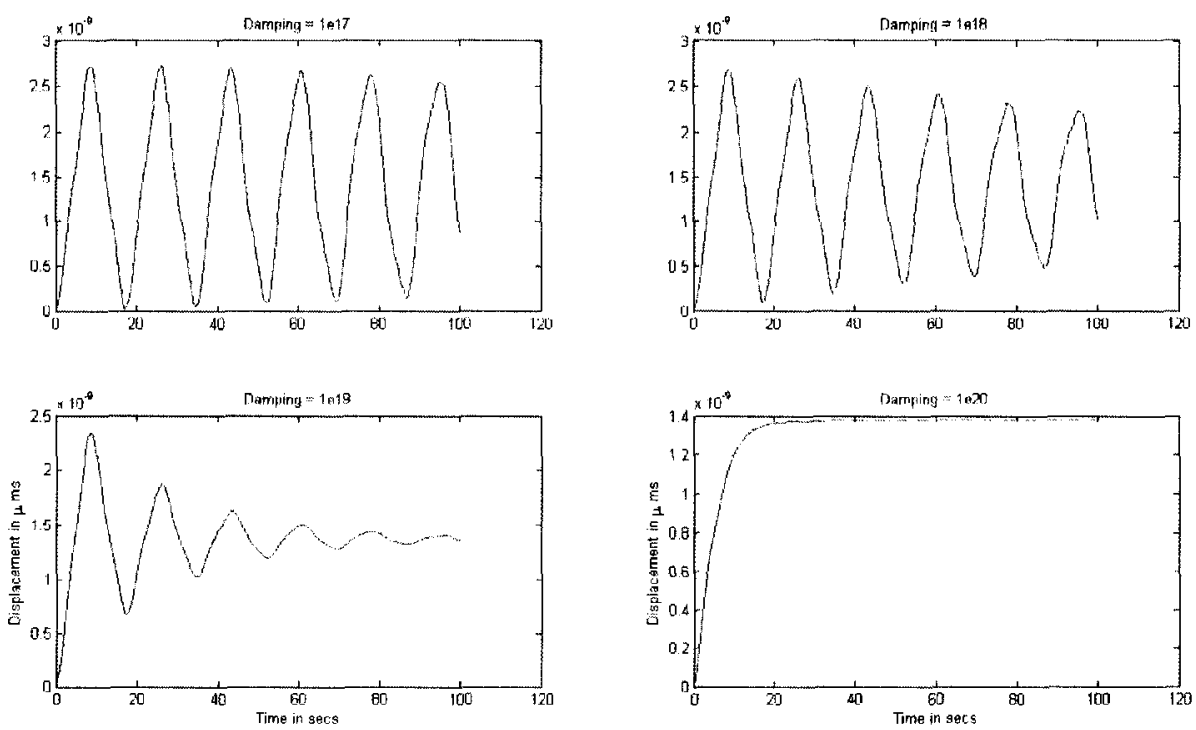

Figure 7.24: Relative error of 150 -element SUGAR Model used to verify $Q$ formed with $D=0$, with SOAR

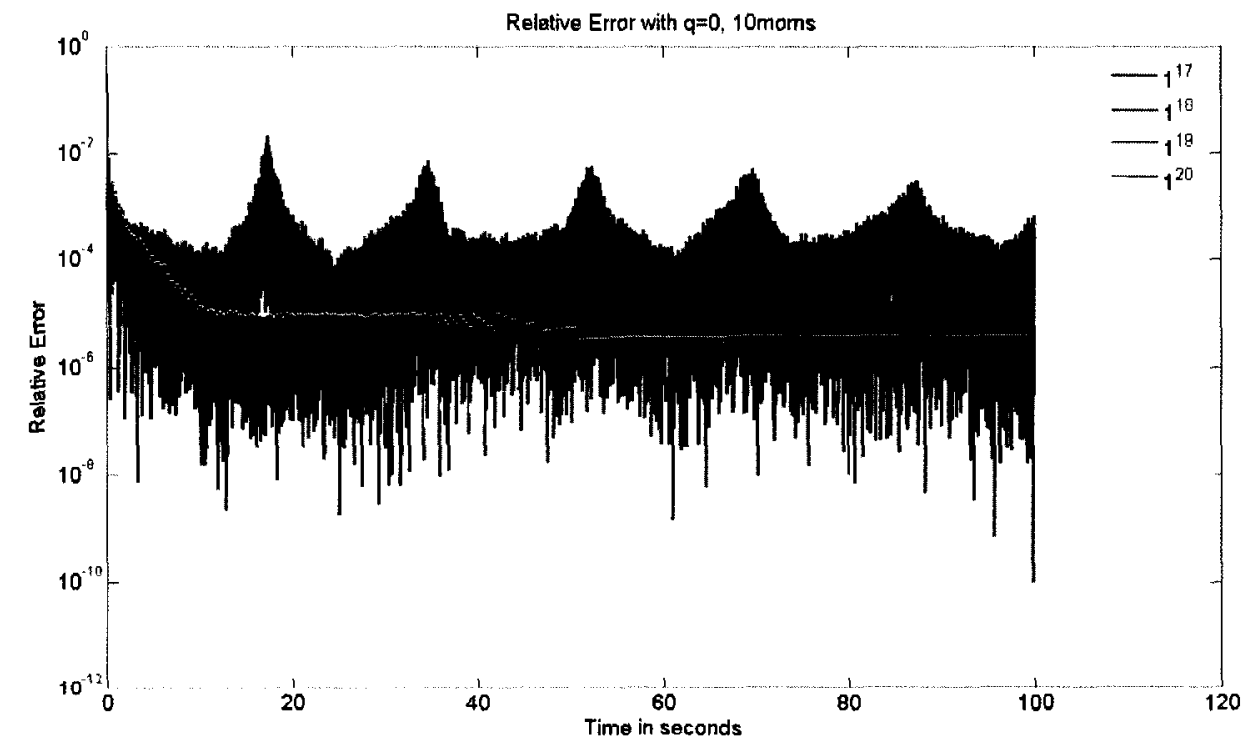

Figure 7.25: Relative error of 150-element SUGAR Model used to verify $Q$ formed with $D=0$, with SOAR 


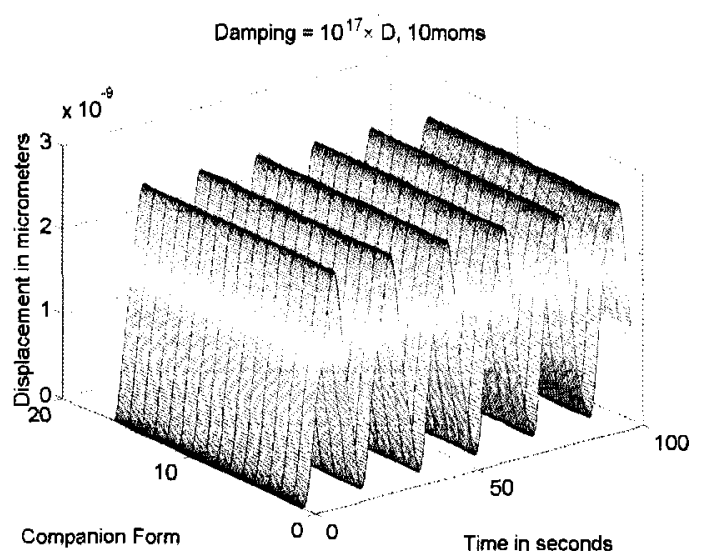

(a)

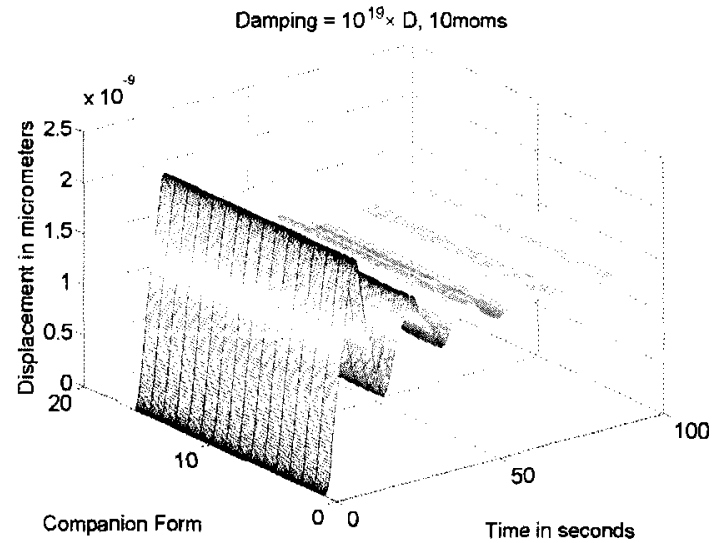

(c)

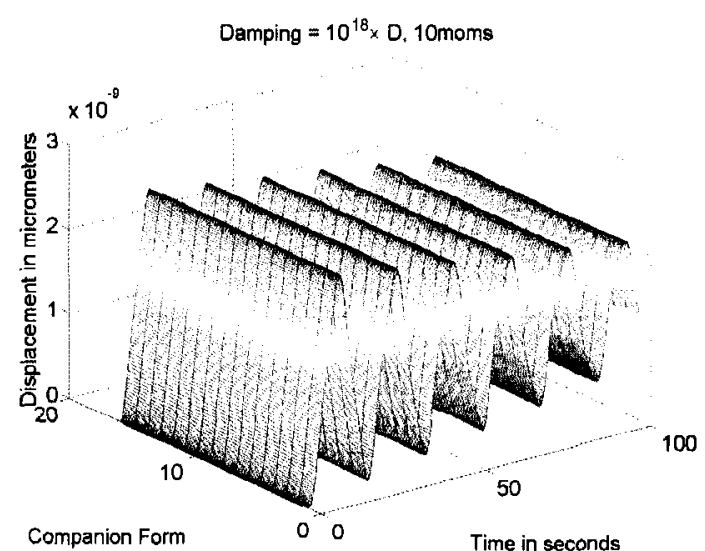

(b)

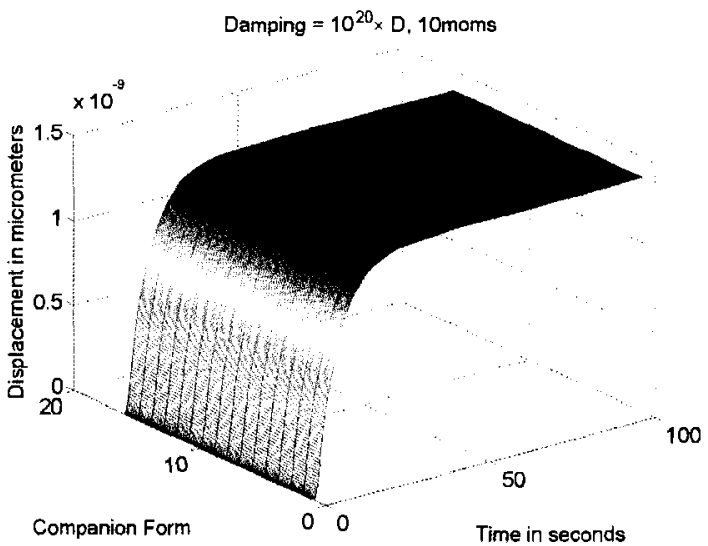

(d)

Figure 7.26: Time domain response of a 150-element SUGAR Model used to verify $Q$ formed with $\mathrm{D}=0$, all 16 companion forms,

Discussion: Clearly, using the undamped model for implementing SOAR and then creating reduced models that were then damped eliminated the instability shown when trying to model structures using the SUGAR modeling technique in Chapter 6. The match it provided to the full model was exceptional. In addition, implementing model reduction for SUGAR models using the Arnoldi algorithm also provided an excellent match to the full model as shown in Figure 7.26 with very low error, refer to Figure 7.27. 


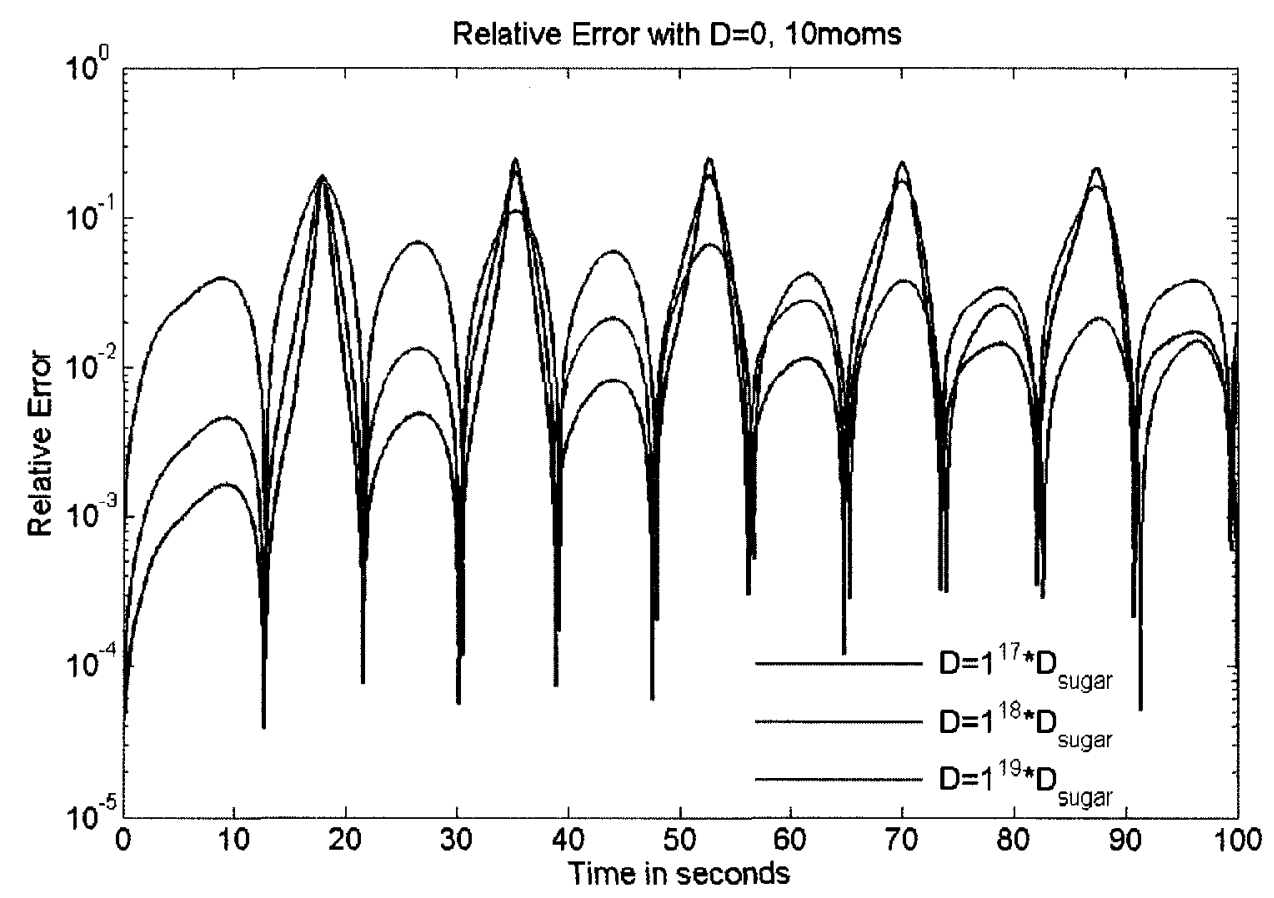

Figure 7.27: Relative error of 150 -element SUGAR Model used to verify $Q$ formed with $D=0$, with Arnoldi

\subsection{Conclusion}

In this chapter the effect of damping on the orthogonal $Q$-matrix was looked at in detail and was found to contribute a negligible amount to this matrix. However, as the damping increased, the contribution scaled by a factor of the damping applied to the system, and as it approached the value for the system to achieve critical damping, instabilities in the response of reduced models generated by using the Arnoldi algorithm were found for all models investigated. A closer look at the way the eigenvalues responded for reduced order systems formed with the Arnoldi algorithm revealed that as the damping increased the eigenvalues became more positive in value and moved closer to the complex axis. This indicated increased damping caused the system to become unstable. 
Another important observation was noted in that the introduction of damping resulted in some loss of orthogonality of eigenvectors of the $Q$ matrix and the transformation $Q^{T} Q=I$ was not an exact match to the identity matrix. The resulting matrix was dense but the non-diagonal values were very small in magnitude when compared with the diagonal elements which all had the value 1 .

In light of these observations, and the extensive analysis carried out, it was clearly seen that forming the orthogonal matrix, $Q$, with no damping and then including the damping when transforming the original system matrices to the reduced matrices has been shown to provide excellent approximations to the real full system despite increasing complexity and varying the value of damping that the reduced model was created with.

This result eliminated any instability that could occur in performing model reduction using either the SOAR procedure or the Arnoldi algorithm with the need for a very small number of moments to achieve acceptable approximations to the real system.

In addition, a designer would only need to perform one model reduction procedure by setting the damping to zero, and then be able to perform tests on structures or devices with varying damping, knowing that an accurate and stable response is always achieved.

As a result, the model reduction technique is much more robust, highly accurate and needs very little moments, and reduces the amount of computational effort that would be required for multiple simulations. 


\section{Chapter 8}

\section{Conclusion}

The main goal of this work was to compare the performance of Krylov based model reduction techniques when applied to modeling the mathematical component of MEMS. These MEMS are conveniently modeled using a second-order differential equation. However, most existing model reduction techniques are based on modeling first order systems, not second-order or higher. Theoretically, the model reduction techniques can be extended to include higher order systems by using a mathematical reformulation which rewrites the higher order system to a first order companion form. The Arnoldi algorithm based on the Krylov subspace methods uses this companion form.

In examining the stability of using this reformulation technique, it was found that, while the companion form was formed to meet the mathematical stability and passivity criteria outlined in Chapter 4, only two of the equivalent sixteen possible companion forms for the second-order systems met these criteria. This was further confirmed in the numerical analysis of a FEM model in the time domain.

A complete numerical analysis in the frequency and time domain was then performed on 
the two companion forms using the Arnoldi algorithm as the model reduction technique. A different model reduction technique, the SOAR procedure, was tested as well. Unlike the Arnoldi algorithm, SOAR performs model reduction through a projection to a second-order Krylov subspace directly so the original second-order form of MEMS can be used. The frequency domain analysis established that both the SOAR procedure and the Arnoldi algorithm performed comparably for FEM models, however the Arnoldi algorithm performed better for SUGAR models, as models created using SUGAR seem to be highly ill-conditioned and the Arnoldi algorithm can overcome ill-conditioning issues by scaling the matrices appropriately, while SOAR cannot. An intriguing result was also noted in that companion forms that did not meet stability criteria also performed similarly to the forms that did pass. This indicated that the frequency domain analysis may fail to capture unstable modes that cannot be used for transient simulations.

The time domain analysis was more interesting for many reasons. The first being that researchers often overlook the time domain analysis because it can require robust time domain simulators, so testing in the time domain is rarely looked at in depth. The second reason is that the time domain analysis revealed some unexpected results. As damping, another overlooked factor in mathematical modeling, changes in structures, the Arnoldi algorithm becomes unstable. This instability was linked to the approach to critical damping of the system, and was present in reduced models formed from the companion forms of the Arnoldi algorithm that met the stability criteria. The SOAR procedure performed exceptionally well for all the structures modeled in FEM, regardless of the damping present. In models created using SUGAR, it was found that SOAR failed to perform when any notable damping was present in the system but Arnoldi was able to achieve stable results if the damping was kept below critical damping. 
The result of the time domain analysis proved that damping does play an important role in the stability of model reduction techniques. Chapter 7 took a closer look at the contribution of damping to reduced models by first looking directly at the moments of the second-order system as damping was varied. Here it was found that damping only contributed to odd-numbered moments, and when it was not present these moments had zero magnitude. The addition of damping caused the introduction of very small numerical values to these odd-numbered moments, usually on the order of $10^{-20}$ less than the even-numbered moments. These contributions were scaled as the damping were scaled, but were enough to cause the system to become unstable at critical damping. Critical damping was found to be approximately $10^{-2} \times M$ for the FEM models examined.

The effect of damping on the stability of reduced models was also examined by looking at the eigenvalues of the reduced system as damping increased, and it was found that as the damping increased the poles moved toward the positive real axis, closer to the complex axis, and became positive at critical damping.

Despite efforts made here, there was no conclusive mathematical reason why critical damping caused this instability, as the system matrices do not become more ill-conditioned. It was observed, however, that introducing damping to a system causes the eigenvectors of the orthogonal $Q$-matrix, formed during model reduction, to have a very small loss of orthogonality, but this loss is on the order of $10^{-17}$ and compared to the diagonal elements which had a magnitude of one, is insignificant. There was also no clear reason as to why at critical damping, this might become significant, as any introduction of damping generates the same effect, the magnitude of which does not define a pattern. 
Finally, based on the moment analysis on the contribution of damping and how small those contributions were, a different approach to model reduction was investigated. This approach involved constructing the orthogonal basis for model reduction by using absolutely no damping, so the orthogonal $Q$-matrix was formed with zero damping. This zero damped $Q$-matrix was then used to transform the original system matrix to the reduced models but with damping included in the mapping.

As the numerical testing performed in the time domain yielded the most instabilities in the reduced order models, all the analysis done here was only done in the time domain.

In this analysis, it was found that with an orthogonal matrix formed with zero damping, it did not matter which of the 16 companion forms were initially used in the reformulation of the second-order system to the first order companion form. It also did not matter which form was selected to then transform the original matrices to reduced ones using the Arnoldi algorithm. They all yielded stable results that matched the full response of the system with extremely low relative error. This was also found to be true when the same methodology was used with the SOAR procedure.

The models were then subjected to varying damping using Rayleigh damping, which ranged from no damping to completely overdamped, and all of the responses matched the full response, again with very low error.

To try to conclusively prove this was a viable and more robust technique, complex structures were then tested and again the results all matched the full response with low error.

Finally, an orthogonal undamped $Q$-matrix was tested on the SUGAR models that could not yield any stable results with SOAR, and here it performed exceptionally well. 
In conclusion, after extensive analysis, and demonstrating the instabilities and lack of robustness still present in model reduction techniques, a variation on performing model reduction was introduced that had a significant number of benefits. These include:

1. Structural preservation of the second-order system even if the system is reformulated to a first order companion form as having no damping means that all the 16 companion forms for the second-order system meet the stability criteria.

2. When using the Arnoldi algorithm, there is never a need to scale the matrices, so trial and error guessing so common in model reduction is eliminated.

3. Very low number of moments can be used even with very large systems. In all the models used here, only 10 moments were needed, even for the complex models with greater than 10000 nodes.

4. This technique performs excellently with all the modeling techniques used here, SUGAR and FEM.

5. Designers only need to construct one orthogonal basis and then vary the damping while transforming the original system matrices to reduced order matrices. Previously, for every damping a new orthogonal matrix would be generated and then mapped to the original system.

6. The analysis indicates that this method is completely robust in every aspect. 


\section{Appendix A}

\section{Moment analysis}

From [21], the poles or eigenvalues of a system is the given as

$$
Y(s)=\sum_{i}^{n} \frac{k_{i}}{s-\lambda_{i}}
$$

Given the second order system

$$
\begin{aligned}
& M \ddot{u}(t)+D \dot{u}(t)+K u(t)=F b(t) \\
& w=L^{T} u(t)
\end{aligned}
$$

By taking the Laplace transform and using a Taylor series expansion, $u(t), \dot{u}(t)$, and $\ddot{u}(t)$ can be rewritten as

$$
\left.\begin{array}{c}
u=m_{0}+m_{1} s+m_{2} s^{2}+m_{3} s^{3}+\ldots .+m_{n} s^{n} \\
\dot{u}=m_{0} s+m_{1} s^{2}+m_{2} s^{3}+m_{3} s^{4}+\ldots .+m_{n} s^{n+1} \\
\ddot{u}=m_{0} s^{2}+m_{1} s^{3}+m_{2} s^{4}+m_{3} s^{5}+\ldots .+m_{n} s^{n+2}
\end{array}\right\}
$$

Substituting equation A.0.3 into equation A.0.2 and equating co-efficients

$$
\left.\begin{array}{c}
M\left(m_{0} s^{2}+m_{1} s^{3}+m_{2} s^{4}+m_{3} s^{5}+\ldots+m_{n} s^{n+2}\right)+ \\
D\left(m_{0} s+m_{1} s^{2}+m_{2} s^{3}+m_{3} s^{4}+\ldots+m_{n} s^{n+1}\right)+ \\
K\left(m_{0}+m_{1} s+m_{2} s^{2}+m_{3} s^{3}+\ldots+m_{n} s^{n}\right)=F \\
K m_{0}=F \\
m_{0} D+m_{1} K=0 \\
m_{0} M+m_{1} D+m_{2} K=0 \\
m_{1} M+m_{2} D+m_{3} K=0
\end{array}\right\}
$$


Rearranging,

$$
\left.\begin{array}{c}
m_{0}=K^{-1} F \\
m_{1}=-K^{-1} D m_{0} \\
m_{2}=-K^{-1} M m_{0}-K^{-1} D m_{1} \\
m_{3}=-K^{-1} M m_{1}-K^{-1} D m_{2} \\
m_{4}=-K^{-1} M m_{2}-K^{-1} D m_{3}
\end{array}\right\}
$$

This gives the relation that

$$
m_{n}=-K^{-1} M m_{n-2}-K^{-1} D m_{n-1}
$$

Let $D=\alpha M+\beta K$,

Equation refapp:7 now becomes

$$
m_{n}=-K^{-1} M\left(m_{n-2}+\alpha m_{n-1}\right)-\beta I m_{n-1}
$$

Rewriting equation A.0.6 in terms of $m_{0}$ and using equation A.0.6

$$
\begin{aligned}
m_{0} & =K^{-1} F \\
m_{1} & =m_{0}\left(-\alpha K^{-1} M-\beta I\right) \\
m_{2} & =-K^{-1} M\left(m_{0}+\alpha m_{1}\right)-\beta m_{1} \\
& =m_{0}\left(-K^{-1} M+\alpha^{2}\left(K^{-1} M\right)^{2}+2 \alpha \beta\left(K^{-1} M\right)^{2}+\beta^{2} I\right) \\
m_{3} & =-K^{-1} M\left(m_{1}+\alpha m_{2}\right)-\beta m_{2} \\
& =m_{0}\left(2 \alpha\left(K^{-1} M\right)^{2}-\alpha^{3}\left(K^{-1} M\right)^{3}-3 \alpha^{2} \beta\left(K^{-1} M\right)^{2}-3 \alpha \beta^{2} K^{-1} M+2 \beta K^{-1} M-\beta^{3}\right)
\end{aligned}
$$




\section{Appendix B}

\section{Analysis of an Analytical Cantilever Beam}

This derivation of a cantilever with an impulse force applied to the free end is taken from [66], [67]

The general equation that governs the cantilever beam is as follows

$$
E_{m} I_{m} \frac{\partial^{4} y(x, t)}{\partial x^{4}}+m \frac{\partial^{2} y(x, t)}{\partial t^{2}}=q(x, t)
$$

For a cantilever with constant cross-sectional area, the equation of the subsequent vibration of the beam after the pulse is applied is

$$
E_{m} I_{m} \frac{\partial^{4} y(x, t)}{\partial x^{4}}+m \frac{\partial^{2} y(x, t)}{\partial t^{2}}=0
$$

where $E_{m}$ is the Young's modulus, $I_{m}$ is the moment of inertia, $m$ is the mass of the beam.

Equation B.0.2 can be solved by using separation of variables method with the assumption that the displacement can be separated into two parts where one is dependent on time and the other on the position of the bar. 
To this end, assume the general solution of equation B.0.1 has the form

$$
y(x, t)=\sum_{n=1}^{\infty} X_{n}(x) f_{n}(t)
$$

where the $X_{n}(x)$ functions are the characteristic functions that satisfy the differential equations

$$
E I \frac{\partial^{4} X_{n}(x)}{\partial x^{4}}=\hat{\omega}_{n}^{2} m X_{n}(x)
$$

where $n=1,2 \ldots, \hat{\omega}_{n}^{2}$ defines the $n^{\text {th }}$ damped natural frequency of the cantilever using standard boundary conditions of the beam since it is fixed at one end such that there is zero displacement, zero shearing force and zero bending moment, that is

$$
\begin{aligned}
& y(0, t)=0 ; \\
& \frac{\partial^{2} y(L, t)}{\partial x^{2}}=0 ; \\
& \frac{\partial y(0, t)}{\partial x}=0 ; \\
& \frac{\partial^{3} y(L, t)}{\partial x^{3}}=0 ;
\end{aligned}
$$

for $t \geq 0$, and where $L$ is the length of the beam

Substituting equation B.0.3 into equation B.0.1 gives

$$
E I \sum_{n=1}^{\infty} \frac{\partial^{4} X_{n}(x)}{\partial x^{4}} f_{n}(t)=q(x, t)-m \sum_{n=1}^{\infty} X_{n}(x) \frac{\partial^{2} f_{n}(t)}{\partial t^{2}}
$$

Rearranging equations B.0.5 and B.0.4

$$
E I \sum_{n=1}^{\infty} \hat{\omega}_{n}^{2} X_{n}(x) f_{n}(t)=\frac{1}{m} q(x, t)-\sum_{n=1}^{\infty} X_{n}(x) \frac{\partial^{2} f_{n}(t)}{\partial t^{2}}
$$

Now, multiply equation B.0.6 on both sides by $X_{m}(x)$ where $m=1,2,3$. and integrate between 0 and $L$. According to orthogonality conditions there exists

$$
\int_{0}^{L} X_{n}(x) X_{m}(x) d x=0
$$


which occurs when $n \neq m$

and,

$$
\int_{0}^{L} X_{n}(x) X_{m}(x) d x=\frac{L}{2}
$$

occurs when $n=m$

Equation B. 0.6 becomes

$$
\frac{\partial^{2} f_{n}(x, t)}{\partial t^{2}}+\hat{\omega}_{n}^{2} f_{n}(x, t)=\frac{2}{m L} Q_{n}(t)
$$

where $n=1,2$,

and

$$
\begin{gathered}
Q_{n}(x)=\int_{0}^{L} p(x, t) X_{n}(x) d x \\
f_{n}(t)=A_{n} \cos \left(\hat{\omega}_{n} t\right)+B_{n} \sin \left(\hat{\omega}_{n} t\right)+\frac{2}{m L \hat{\omega}_{n}} \int_{0}^{t} Q(\tau) \sin \hat{\omega}_{n}(t-\tau) d \tau \\
w(x, t)=\sum_{0}^{\infty} X_{n}(x) f_{n}(t)=\sum_{0}^{\infty}\left[A_{n} \cos \left(\hat{\omega}_{n} t\right)+B_{n} \sin \left(\hat{\omega}_{n} t\right)+\frac{2}{m L \hat{\omega}_{n}} \int_{0}^{t} Q(\tau) \sin \hat{\omega}_{n}(t-\tau) d \tau\right] X_{n}(x)
\end{gathered}
$$

If the initial conditions of zero deflection $y=0$ at $t=0$ are assumed, then

Equation equation B.0.12 now becomes

$$
w(x, t)=\sum_{0}^{\infty}\left[\frac{2}{m L \hat{\omega}_{n}} \int_{0}^{t} Q(\tau) \sin \hat{\omega}_{n}(t-\tau) d \tau\right] X_{n}(x)
$$

Now, finding the general solution to the homogeneous equation B.0.2

Dividing equation B. 0.2 by $y(x, t)=X(x) f(t)$, to get,

$$
\frac{E_{m} I_{m}}{m X(x)} \frac{\partial^{4} y(x, t)}{\partial x^{4}}=-\frac{1}{f(t)} \frac{\partial^{2} y(x, t)}{\partial t^{2}}=\hat{\omega}^{2}
$$


where $\hat{\omega}^{2}$ is a constant

Following from equation B.0.8 the two differential equations that satisfied by $X(x)$ and $f(t)$

are

$$
\begin{gathered}
E_{m} I_{m} \frac{\partial^{4} X(x)}{\partial x^{4}}-\omega^{2} m \frac{\partial^{2} X(x)}{\partial t^{2}}=0 \\
\frac{\partial^{2} f(t)}{\partial t^{2}}+\hat{\omega}^{2} f(t)=0
\end{gathered}
$$

The general solution of equation B.0.10 is then given by

$$
f(t)=A \cos (\hat{\omega} t)+A \sin (\hat{\omega} t)
$$

Since the bar is assumed to have constant cross-sectional area

$$
\frac{\partial^{4} X(x)}{\partial x^{4}}-k^{4} X=0
$$

where

$$
k^{2}=\sqrt{\frac{\hat{\omega}^{2} m}{E I}}
$$

The general solution to equation B.0.15 is

$X(x)=C_{1}\left[\cos \left(k_{n} x\right)+\cosh \left(k_{n} x\right)\right]+C_{2}\left[\cos \left(k_{n} x\right)-\cosh \left(k_{n} x\right)\right]+C_{3}\left[\sin \left(k_{n} x\right)+\sinh \left(k_{n} x\right)\right]+C_{4}\left[\sin \left(k_{n} x\right)\right.$

where $C_{1}, C_{2}, C_{3}, C_{4}$ are arbitrary constants

Using boundary conditions outlined above we can solve for these constants such that

$$
\begin{aligned}
& X(0)=0=C_{1}[2] \Rightarrow C_{1}=0 \\
& \frac{\partial X(0)}{\partial x}=C_{2}\left[-\sin \left(k_{n} x\right)-\sinh \left(k_{n} x\right)\right]+C_{3}\left[\cos \left(k_{n} x\right)+\cosh \left(k_{n} x\right)\right]+C_{4}\left[\cos \left(k_{n} x\right)-\cosh \left(k_{n} x\right)\right] \\
& \frac{\partial X(0)}{\partial x}=0=C_{3}[2] \Rightarrow C_{3}=0 \\
& \frac{\partial^{2} X(L)}{\partial x^{2}}=C_{2}\left[-\cos \left(k_{n} L\right)-\cosh \left(k_{n} L\right)\right]+C_{4}\left[-\sin \left(k_{n} L\right)-\sinh \left(k_{n} L\right)\right]=0 \\
& \frac{\partial^{3} X(L)}{\partial x^{3}}=C_{2}\left[\sin \left(k_{n} L\right)-\sinh \left(k_{n} L\right)\right]+C_{4}\left[-\cos \left(k_{n} L\right)-\cosh \left(k_{n} L\right)\right]=0
\end{aligned}
$$


Other constants can be found similarly giving the final solution with the form

$$
X_{n}(x)=C_{2}\left\{\left[\cos \left(k_{n} x\right)-\cosh \left(k_{n} x\right)\right]+\left[\frac{\left[-\cos \left(k_{n} L\right)-\cosh \left(k_{n} L\right)\right]}{\left[\sin \left(k_{n} L\right)+\sinh \left(k_{n} L\right)\right]}\right]\left[\sin \left(k_{n} x\right)-\sinh \left(k_{n} x\right)\right]\right\}
$$

Recall the solution of the differential equation is

$$
w(x, t)=\sum_{0}^{\infty}\left[\frac{2}{m L \hat{\omega}_{n}} \int_{0}^{t} Q(\tau) \sin \hat{\omega}_{n}(t-\tau) d \tau\right] X_{n}(x)
$$

and that,

$$
Q_{n}(x)=\int_{0}^{L} p(x, t) X_{n}(x) d x
$$

For a step-function applied to the beam at $x=L$, where $L$ is the length of the beam

$$
Q_{n}\left(x\left\{\left[\cos \left(k_{n} L\right)-\cosh \left(k_{n} L\right)\right]+\left[\frac{\left[-\cos \left(k_{n} L\right)-\cosh \left(k_{n} L\right)\right]}{\left[\sin \left(k_{n} L\right)+\sinh \left(k_{n} L\right)\right]}\right]\left[\sin \left(k_{n} L\right)-\sinh \left(k_{n} L\right)\right]\right\}\right.
$$

Simplifying this becomes

$$
\begin{gathered}
Q_{n}(x)=\left[\frac{\left[-2 \cos \left(k_{n} L\right) \sinh \left(k_{n} L\right)+2 \sin \left(k_{n} L\right) \cosh \left(k_{n} L\right)\right]}{\left[\sin \left(k_{n} L\right)+\sinh \left(k_{n} L\right)\right]}\right] \\
w(x, t)=\sum_{0}^{\infty}\left[\frac{2}{m L \hat{\omega}_{n}} \int_{0}^{t} \frac{\left[-2 \cos \left(k_{n} L\right) \sinh \left(k_{n} L\right)+2 \sin \left(k_{n} L\right) \cosh \left(k_{n} L\right)\right]}{\left[\sin \left(k_{n} L\right)+\sinh \left(k_{n} L\right)\right]} \sin \hat{\omega}_{n}(t-\tau) d \tau\right] X_{n}(x) \\
w(x, t)=\sum_{0}^{\infty}\left[\frac{4}{m L \hat{\omega}_{n}^{2}} * \frac{\left[-\cos \left(k_{n} L\right) \sinh \left(k_{n} L\right)+\sin \left(k_{n} L\right) \cosh \left(k_{n} L\right)\right]}{\left[\sin \left(k_{n} L\right)+\sinh \left(k_{n} L\right)\right]} *\left(1-\cos \left(\hat{\omega}_{n} t\right)\right)\right] X_{n}(x)
\end{gathered}
$$

The complete solution of the system is then

$$
\begin{aligned}
& w(x, t)=\sum_{0}^{\infty}\left[\frac{4}{m L \hat{\omega}_{n}^{2}} * \frac{\left[-\cos \left(k_{n} L\right) \sinh \left(k_{n} L\right)+\sin \left(k_{n} L\right) \cosh \left(k_{n} L\right)\right]}{\left[\sin \left(k_{n} L\right)+\sinh \left(k_{n} L\right)\right]} *\left(1-\cos \left(\hat{\omega}_{n} t\right)\right)\right] * \\
& {\left[\cos \left(k_{n} x\right)-\cosh \left(k_{n} x\right)\right]+\left[\frac{\left[-\cos \left(k_{n} L\right)-\cosh \left(k_{n} L\right)\right]}{\left[\sin \left(k_{n} L\right)+\sinh \left(k_{n} L\right)\right]}\right]\left[\sin \left(k_{n} x\right)-\sinh \left(k_{n} x\right)\right]}
\end{aligned}
$$


The equation of motion is re-written to include damping as follows

$$
M_{i} \ddot{q}_{i}+C_{i} \dot{q}_{i}+K_{i} q_{i}=P_{i}
$$

The damping ratio is defined as,

$$
\varsigma_{i}=\frac{C_{i}}{2 M_{i} \omega_{i}}=\frac{C_{i}}{C_{c r}}
$$

where $C_{c r}$ is the critical damping of the system given as

$$
\begin{gathered}
C_{c r}=2 \sqrt{M_{i} K_{i}} \\
\ddot{q}_{i}+2 \varsigma_{i} \omega_{i} \dot{q}_{i}+\omega_{i}^{2} q_{i}=\frac{P_{i}}{M_{i}}
\end{gathered}
$$

The general solution to the equation of motion is,

$$
\dot{q}_{i}=A_{1} e^{\alpha_{1} t}+A_{2} e^{\alpha_{2} t}
$$

Finally, the time-domain response of an analytical cantilever is given as

$$
q_{i}=e^{-\varsigma_{i} w_{i} t}\left[\frac{\dot{q}_{i}(0)+q_{i}(0)}{\omega_{i} \sqrt{1-\varsigma_{i}^{2}}} \sin \left(\omega_{i} \sqrt{1-\varsigma_{i}^{2}} t\right)+q_{i}(0) \cos \left(\left(\omega_{i} \sqrt{1-\varsigma_{i}^{2}} t\right)\right]+\frac{1}{M_{i}\left(\omega_{i} \sqrt{1-\varsigma_{i}^{2}}\right)} \int_{0}^{t} P_{i}(\tau)\right.
$$

The first part of the solution

$$
e^{-\varsigma_{i} w_{i} t}\left[\frac{\dot{q}_{i}(0)+q_{i}(0)}{\omega_{i} \sqrt{1-\varsigma_{i}^{2}}} \sin \left(\omega_{i} \sqrt{1-\varsigma_{i}^{2}} t\right)+q_{i}(0) \cos \left(\left(\omega_{i} \sqrt{1-\varsigma_{i}^{2}} t\right)\right]\right.
$$

goes to zero as, as outlined above, the initial conditions assumes initial displacement and velocity are both 0 .

Therefore, the solution for the forced response now becomes

$$
w_{n} x(t)=\sum_{n=1}^{\infty}\left[\frac{1}{M_{n} l\left(\omega_{n} \sqrt{1-\varsigma_{n}^{2}}\right)} \int_{0}^{t} P_{n}(\tau) e^{-\varsigma_{n} \omega_{n}(t-\tau)} \sin \left(\omega_{n} \sqrt{1-\varsigma_{n}^{2}}(t-\tau)\right) d \tau\right] X_{n}(x)
$$


For a step function applied at $x=L$,

$$
\begin{aligned}
& X_{n}(L)=\left[\cos \left(k_{n} L\right)-\cosh \left(k_{n} L\right)\right]+\left[\frac{\left[-\cos \left(k_{n} L\right)-\cosh \left(k_{n} L\right)\right]}{\left[\sin \left(k_{n} L\right)+\sinh \left(k_{n} L\right)\right]}\right]\left[\sin \left(k_{n} L\right)-\sinh \left(k_{n} L\right)\right]= \\
& X_{n}(L)=\left[\frac{\left[-2 \cos \left(k_{n} L\right) \sinh \left(k_{n} L\right)+2 \sin \left(k_{n} L\right) \cosh \left(k_{n} L\right)\right]}{\left[\sin \left(k_{n} L\right)+\sinh \left(k_{n} L\right)\right]}\right]
\end{aligned}
$$

Since all other terms are now constant values the integral from equation B.0.37 is now

$$
w_{n} x(t)=\frac{1}{M_{n} L\left(\omega_{n} \sqrt{1-\varsigma_{n}^{2}}\right)} * X_{n}(L) \int_{0}^{t} e^{-\varsigma_{n} \omega_{n}(t-\tau)} \sin \left(\omega_{n} \sqrt{1-\varsigma_{n}^{2}}(t-\tau)\right) d \tau
$$

Evaluating

$$
\int_{0}^{t} e^{-\varsigma_{n} \omega_{n}(t-\tau)} \sin \left(\omega_{n} \sqrt{1-\varsigma_{n}^{2}}(t-\tau)\right) d \tau
$$

to get,

$$
\frac{\sqrt{1-\varsigma_{n}^{2}}}{\omega_{n}}\left(1-e^{-\varsigma_{n} \omega_{n}(t-\tau)}\left[\cos \omega_{n} \sqrt{1-\varsigma_{n}^{2}}(t)+\frac{\varsigma_{n} \omega_{n}}{\omega_{n} \sqrt{\left(1-\varsigma_{n}^{2}\right)}} \sin \omega_{n} \sqrt{1-\varsigma_{n}^{2}}(t)\right]\right)
$$

Now substituting into equation B.0.39

$$
\begin{aligned}
& w_{n} x(t)=\frac{1}{M_{n} L\left(\omega_{n} \sqrt{1-\zeta_{n}^{2}}\right)} * \\
& X_{n}(L) \frac{\sqrt{1-\zeta_{n}^{2}}}{\omega_{n}}\left(1-e^{-\varsigma_{n} \omega_{n}(t-\tau)}\left[\cos \omega_{n} \sqrt{1-\varsigma_{n}^{2}}(t)+\frac{\varsigma_{n} \omega_{n}}{\omega_{n} \sqrt{\left(1-\varsigma_{n}^{2}\right)}} \sin \omega_{n} \sqrt{1-\varsigma_{n}^{2}}(t)\right]\right) \\
& w_{n} x(t)=\frac{1}{M_{n} I_{\omega_{n}} \sqrt{\left(1-\zeta_{n}^{2}\right)}} * \\
& X_{n}(L) * \frac{\sqrt{1-\varsigma_{n}^{2}}}{\omega_{n}}\left(1-e^{-\varsigma_{n} \omega_{n}(t-\tau)}\left[\cos \omega_{n} \sqrt{1-\varsigma_{n}^{2}}(t)+\frac{\varsigma_{n} \omega_{n}}{\omega_{n} \sqrt{\left(1-\varsigma_{n}^{2}\right)}} \sin \omega_{n} \sqrt{1-\varsigma_{n}^{2}}(t)\right]\right) \\
& w_{n} x(t)=\frac{2}{M_{n} L \omega_{n}^{2}} * \frac{\left[-\cos \left(k_{n} L\right) \sinh \left(k_{n} L\right)+\sin \left(k_{n} L\right) \cosh \left(k_{n} L\right)\right]}{\left[\sin \left(k_{n} L\right)-\sinh \left(k_{n} L\right)\right]} * \\
& \left(1-e^{-\varsigma_{n} \omega_{n} t}\left[\cos \omega_{n} \sqrt{1-\varsigma_{n}^{2}}(t)+\frac{\varsigma_{n} \omega_{n}}{\omega_{n} \sqrt{\left(1-\varsigma_{n}^{2}\right)}} \sin \omega_{n} \sqrt{1-\varsigma_{n}^{2}}(t)\right]\right) X_{n}(x) \\
& w_{n} x(t)=\frac{2}{M_{n} L\left(\frac{r_{0} o^{4}}{L^{4}}\right)\left(\frac{E_{m} I_{m}}{M_{n}}\right)} * \frac{\left[-\cos \left(k_{n} L\right) \sinh \left(k_{n} L\right)+\sin \left(k_{n} L\right) \cosh \left(k_{n} L\right)\right]}{\left[\sin \left(k_{n} L\right)-\sinh \left(k_{n} L\right)\right]} * \\
& \left(1-e^{-\varsigma_{n} \omega_{n} t}\left[\cos \omega_{n} \sqrt{1-\varsigma_{n}^{2}}(t)+\frac{\varsigma_{n} \omega_{n}}{\omega_{n} \sqrt{\left(1-\varsigma_{n}^{2}\right)}} \sin \omega_{n} \sqrt{1-\varsigma_{n}^{2}}(t)\right]\right) X_{n}(x) \\
& w_{n} x(t)=\text { force } * \frac{2 L^{3}}{\operatorname{root}^{4} * E_{m} * I_{m}} * \frac{\left[-\cos \left(k_{n} L\right) \sinh \left(k_{n} L\right)+\sin \left(k_{n} L\right) \cosh \left(k_{n} L\right)\right]}{\left[\sin \left(k_{n} L\right)-\sinh \left(k_{n} L\right)\right]} * \\
& \left(1-e^{-\varsigma_{n} \omega_{n} t}\left[\cos \omega_{n} \sqrt{1-\varsigma_{n}^{2}}(t)+\frac{\varsigma_{n} \omega_{n}}{\omega_{n} \sqrt{\left(1-\varsigma_{n}^{2}\right)}} \sin \omega_{n} \sqrt{1-\varsigma_{n}^{2}}(t)\right]\right)
\end{aligned}
$$




$$
\begin{gathered}
k_{n} L=\text { root } \\
k_{n}=\frac{\text { root }}{L} \\
\omega_{n}=k_{n}^{2} \sqrt{\frac{E_{m} I_{m}}{M_{n}}}
\end{gathered}
$$

$M_{n}=\frac{\gamma \Omega}{g}$ where $\frac{\gamma}{g}$ is the mass density and $\Omega$ is the cross sectional area of the beam 


\section{Appendix C}

\section{Frequency Response of the Cantilever}

Beam $<10^{-2}$

Further frequency responses for the cantilever beam for dampings of $D=1 \times M$ and lower than $10^{-2} \times M$ is illustrated below.

C.1 1350-node Cantilever Model with Damping $=0.0 \times M$ 


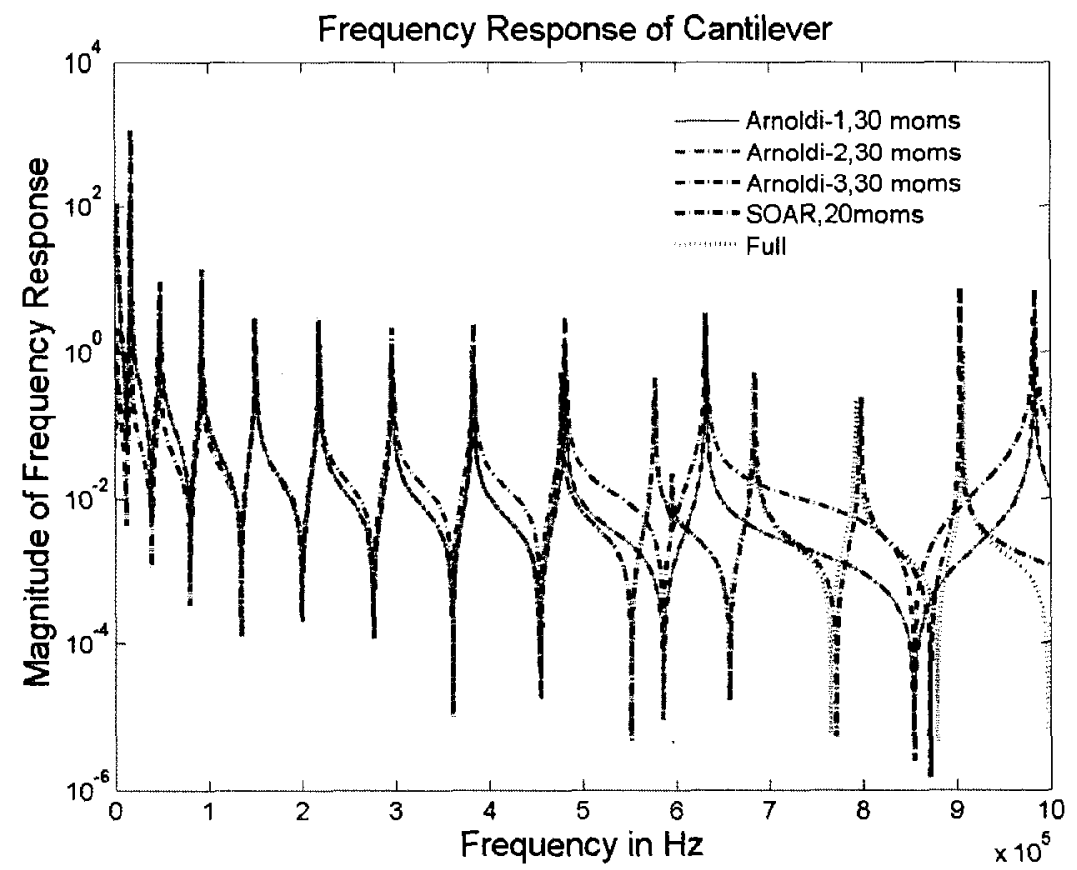

(a) Arnoldi-1, Arnoldi-2, Arnoldi-3, SOAR

Figure C.1: Comparison of frequency response for the full system and reduced model of a 1350 node Cantilever with Damping $=0 \times M$ 
Arnoldi-1

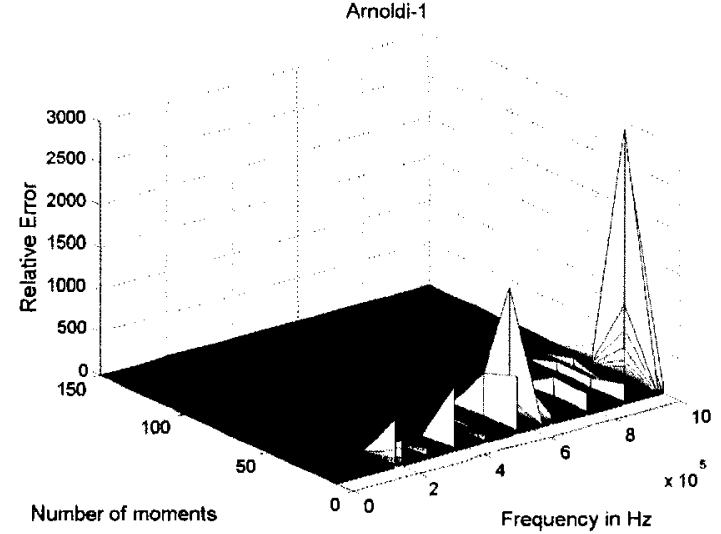

(a) Arnoldi-1

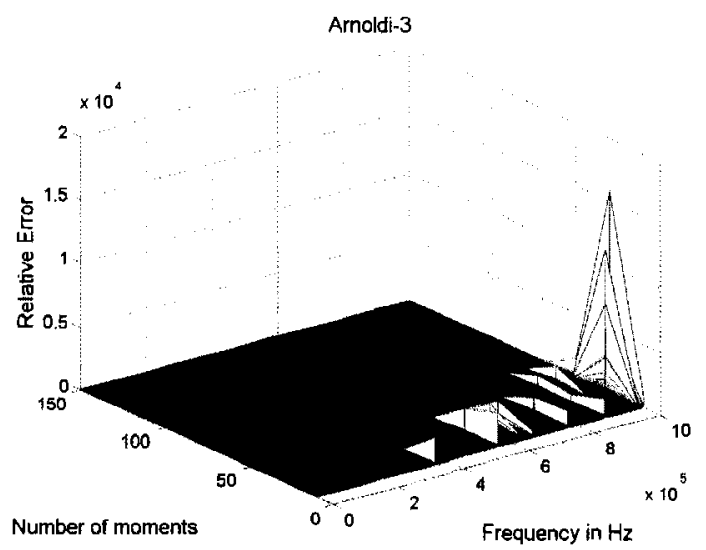

(c) Arnoldi-3

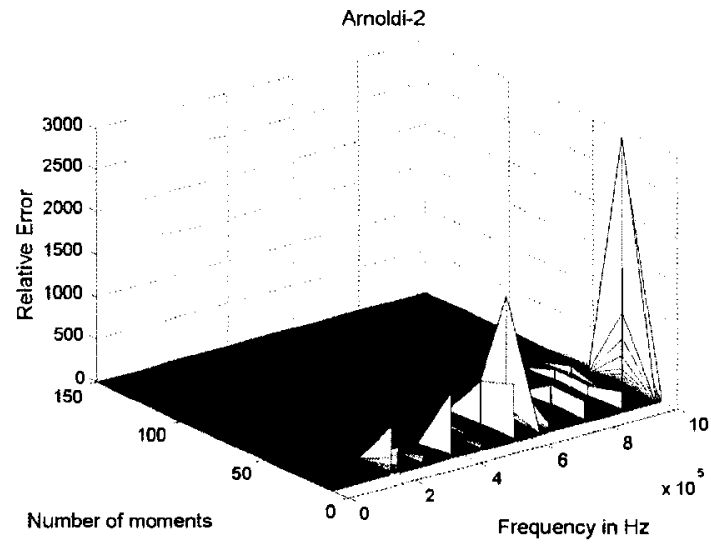

(b) Arnoldi-2

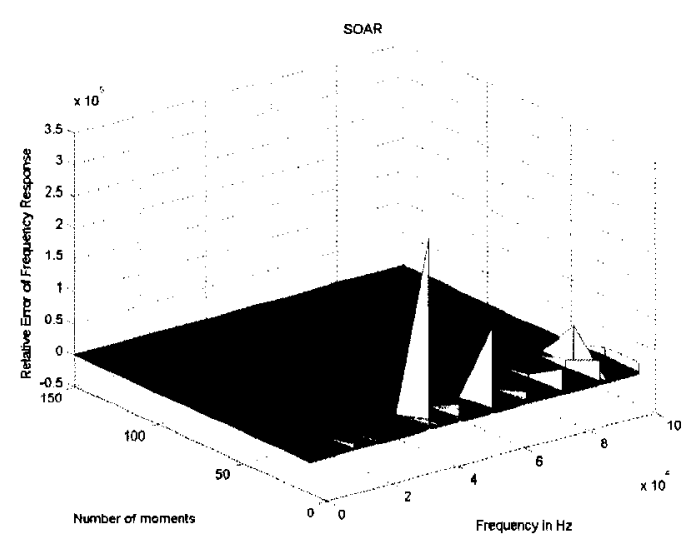

(d) SOAR

Figure C.2: Relative Error for the Frequency Response for 1350-node cantilever,Damping=0 $\times$ M 
Discussion: For the 1350-node cantilever, there was a greater amount of error and greater than 70 moments were needed to keep the relative error low. Again, the cut-off for requiring a larger amount of moments was about $4 \times 10^{5} \mathrm{~Hz}$.

\section{C.2 1350-node Cantilever Model with Damping $=10^{-2} \times M$}

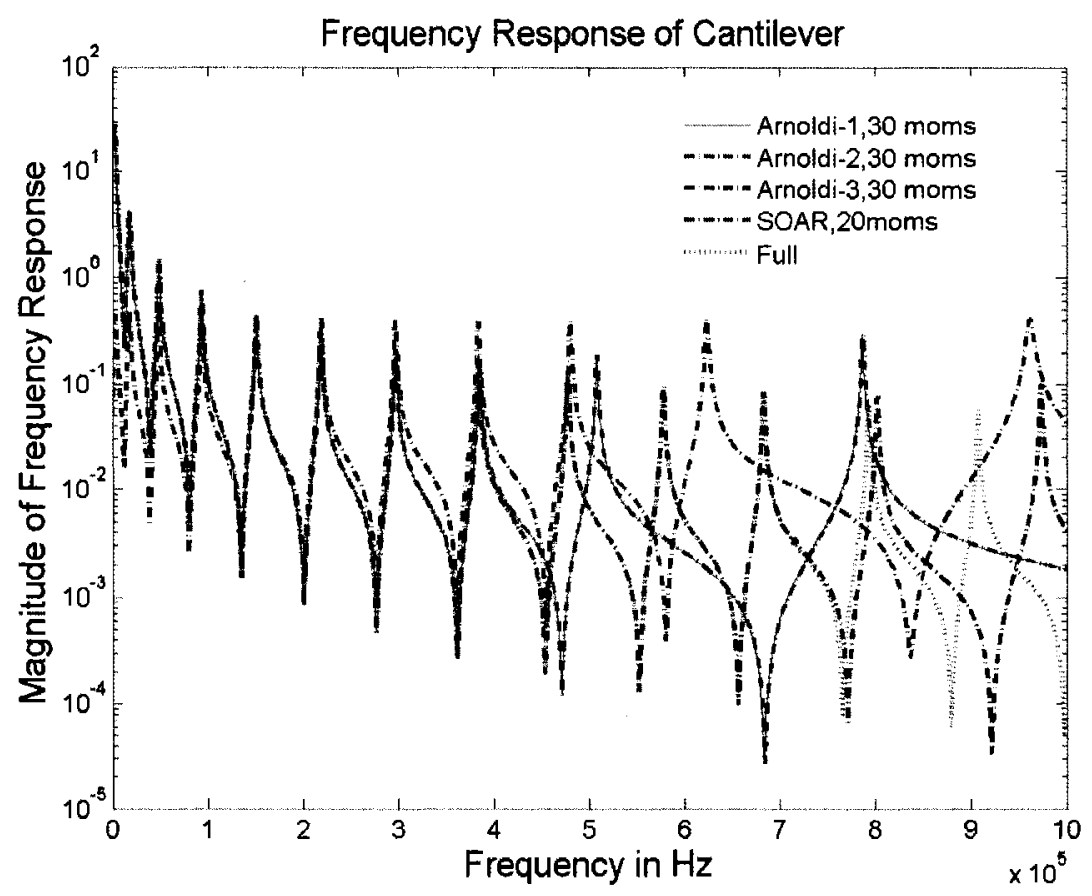

(a) Arnoldi-1, Arnoldi-2, Arnoldi-3, SOAR

Figure C.3: Comparison of frequency responses for the full system and reduced model of a 2700-node Cantilever with Damping $=10^{-2} \times M$ 


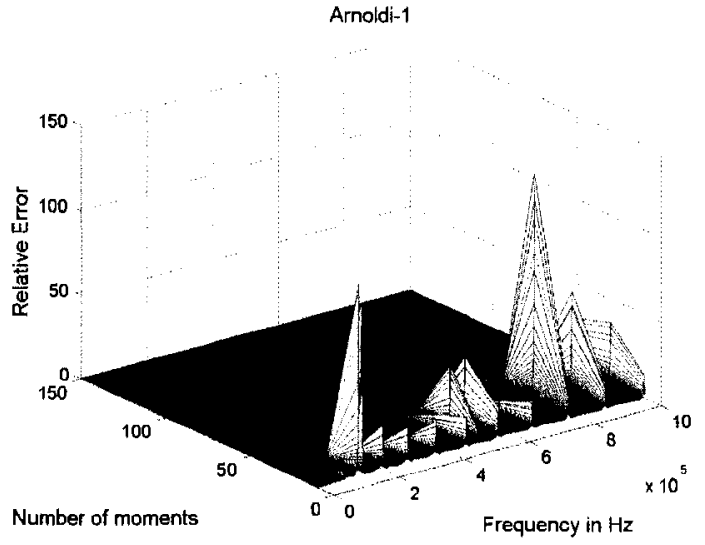

(a) Arnoldi-1

Arnoldi-3

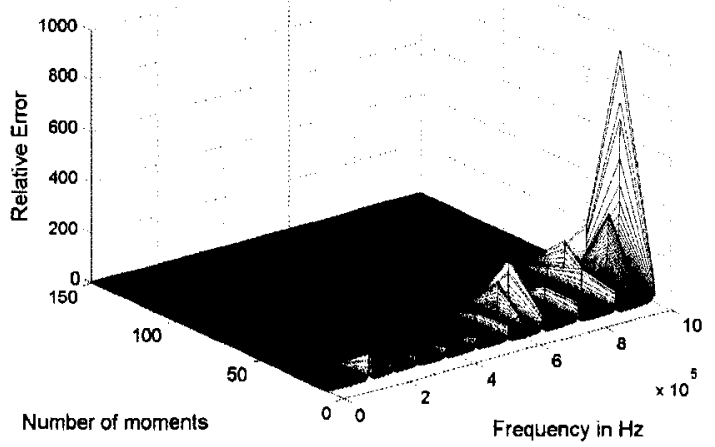

(c) Arnoldi-3

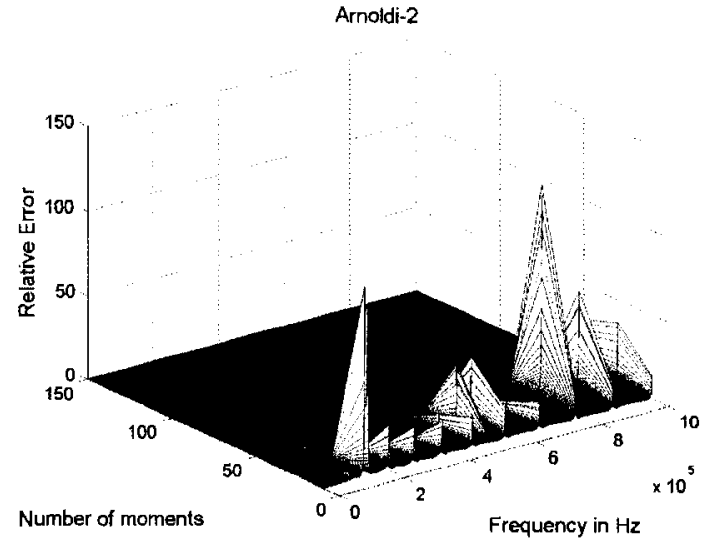

(b) Arnoldi-2

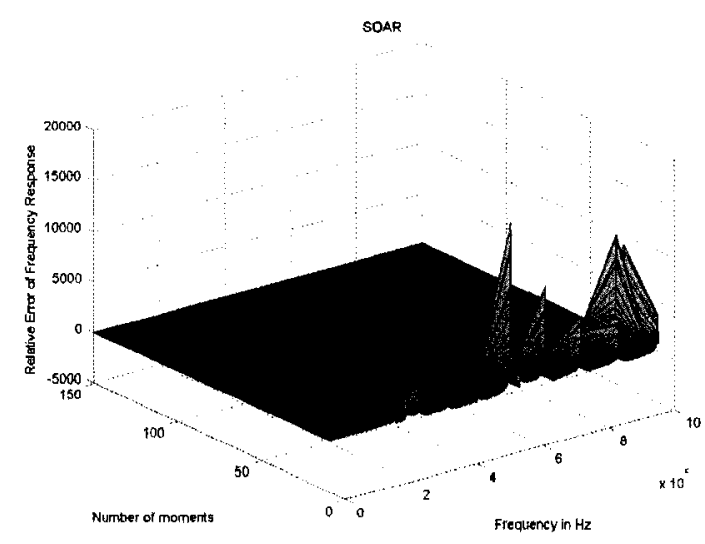

(d) SOAR

Figure C.4: Relative Error for the Frequency Response for 1350-node cantilever,Damping $=10^{-2} \times M$ 
Discussion: The overall frequency response for the 1350-node cantilever was again similar to the 540-node, but the number of moments required was larger to achieve the same relative error.

\section{C.2.1 1350-node Cantilever Model with Damping $=10^{-1} \times M$}

\section{0-node Cantilever}

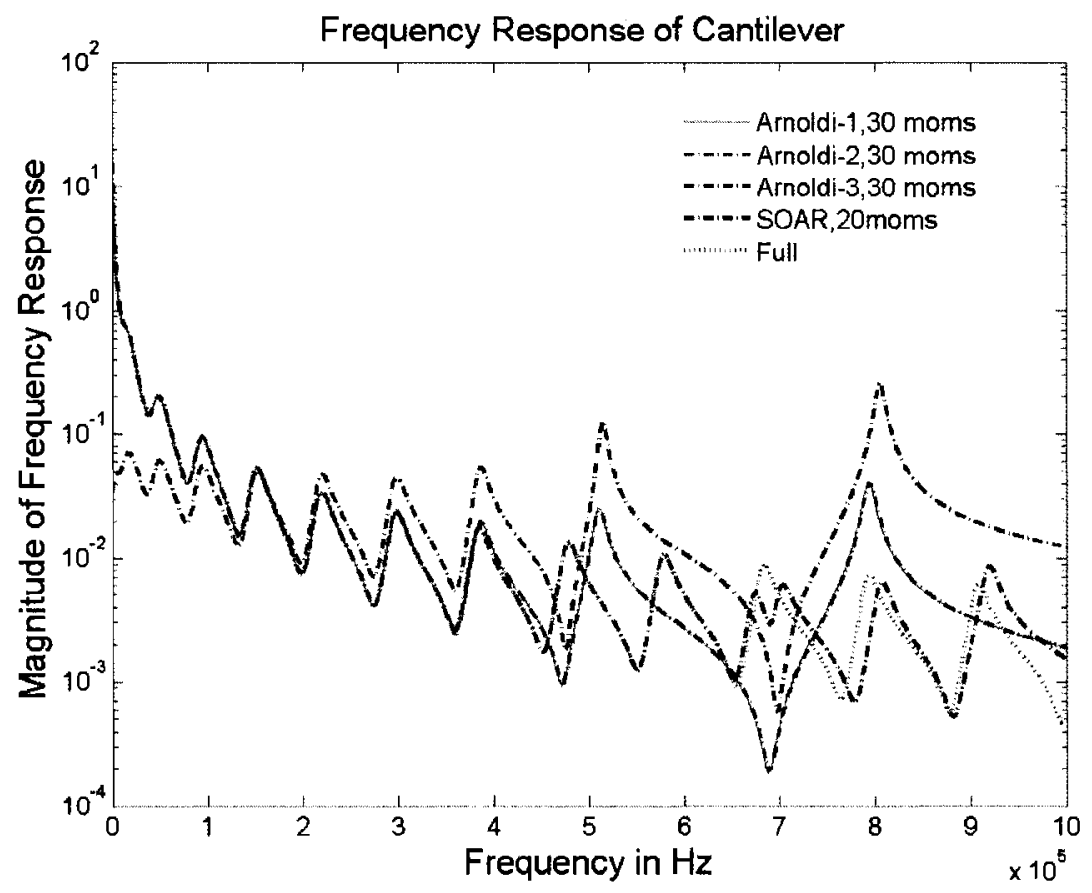

(a) Arnoldi-1, Arnoldi-2, Arnoldi-3, SOAR

Figure C.5: Comparison of frequency response for the full system and reduced model of a 1350 node Cantilever with Damping $=10^{-1} \times M$ 


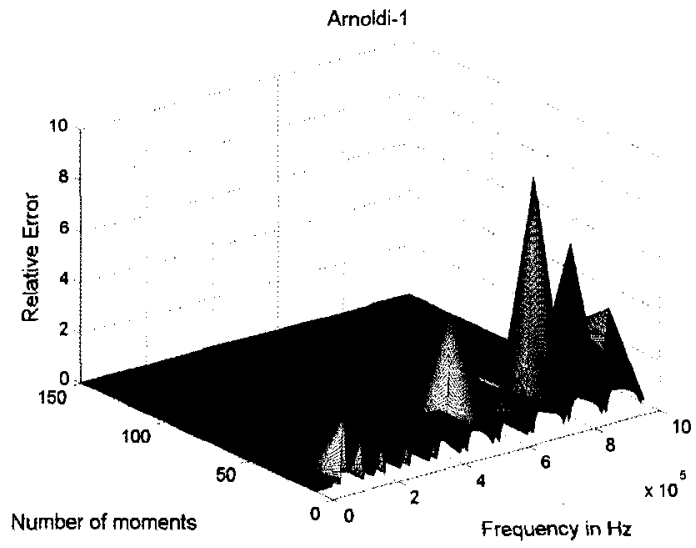

(a) Arnoldi-1

Arnoldi-3

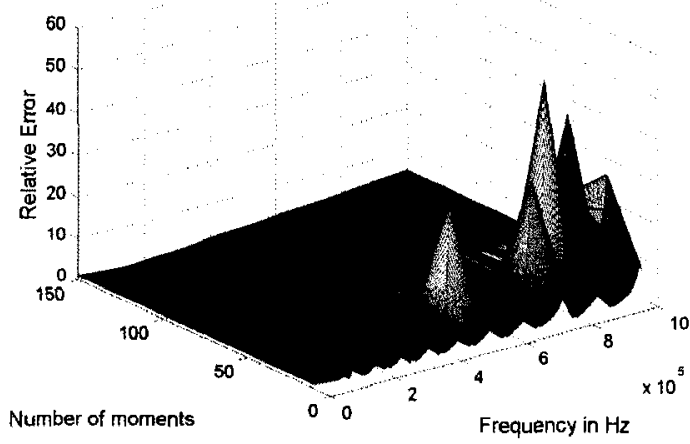

(c) Arnoldi-3

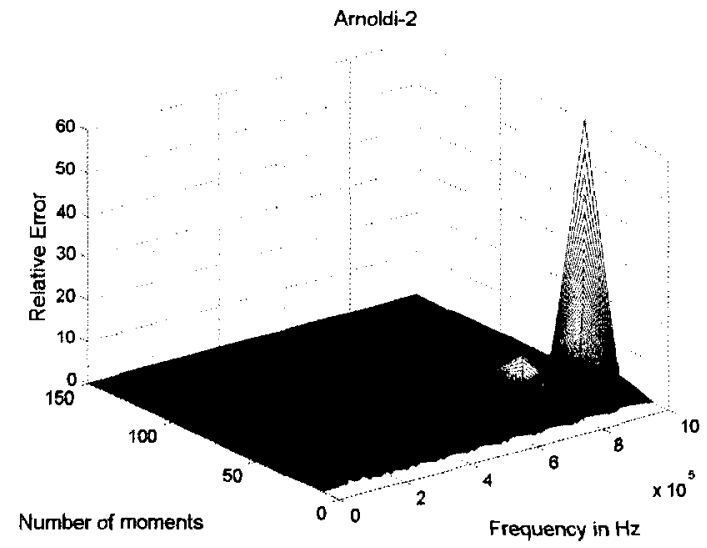

(b) Arnoldi-2

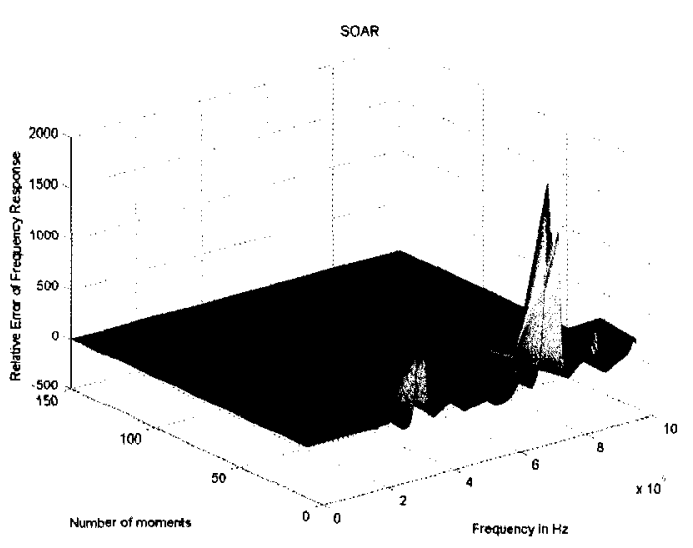

(d) SOAR

Figure C.6: Relative Error for the Frequency Response for 1350-node cantilever,Damping= $10^{-1} \times M$ 
Discussion: The same observations for the 540-node cantilever were made for the 1350-node, but the level of error seen was much higher and a minimum of 30 moments were needed for to maintain a low relative error, as shown in Figure C.6.

\section{C.3 Cantilever Models with Damping $=1.0 \times M$}

\section{C.3.1 540-node Cantilever}

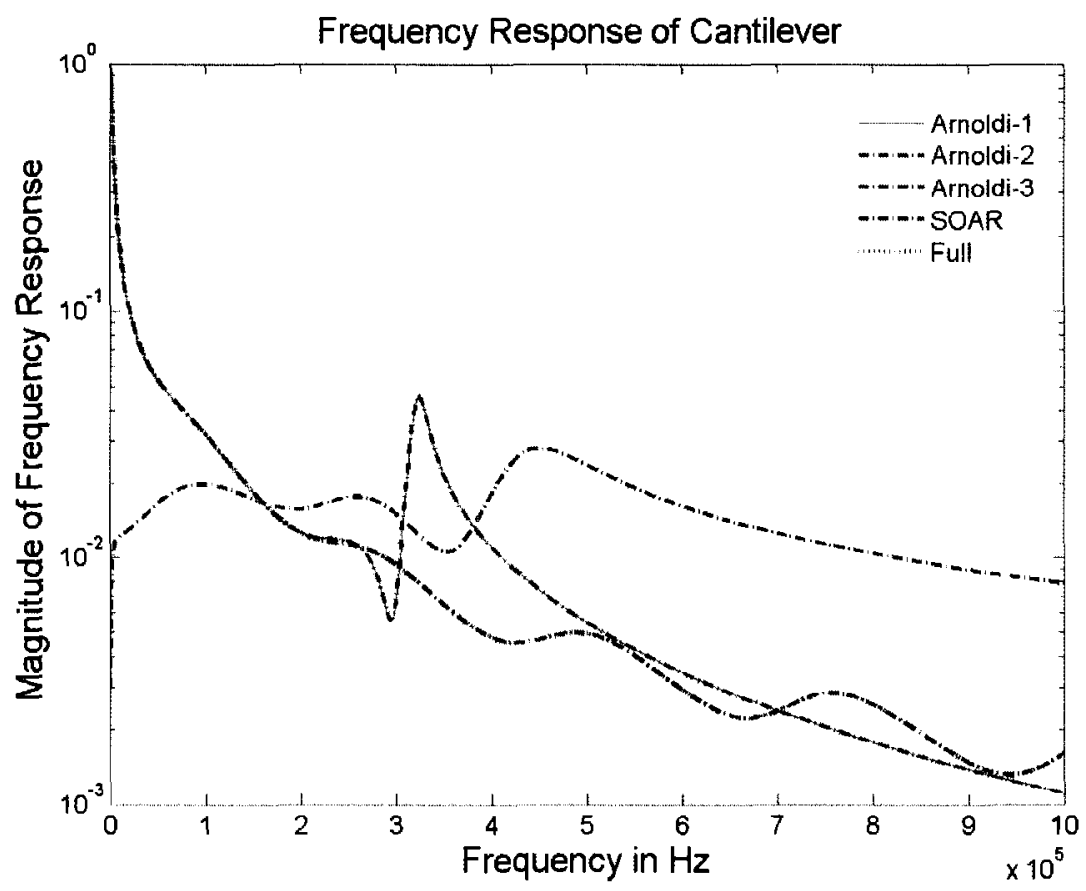

(a) Arnoldi-1, Arnoldi-2, Arnoldi-3, SOAR

Figure C.7: Comparison of frequency responses for the full system and reduced model of a 540 node Cantilever with Damping $=1.0 \times M$ 


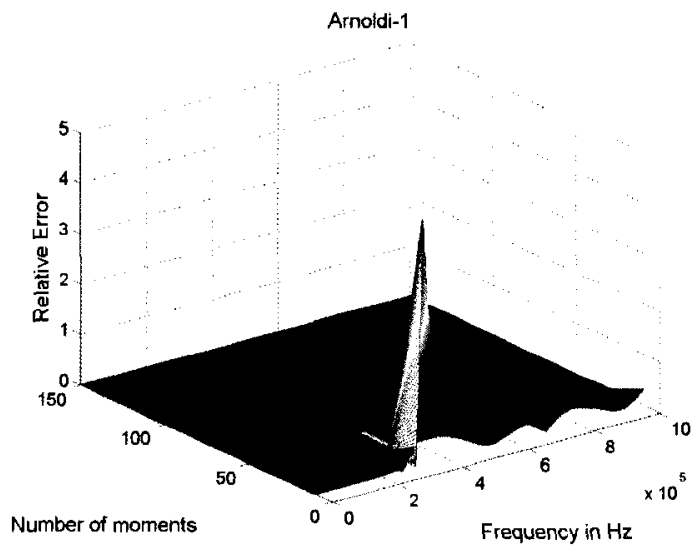

(a) Arnoldi-1

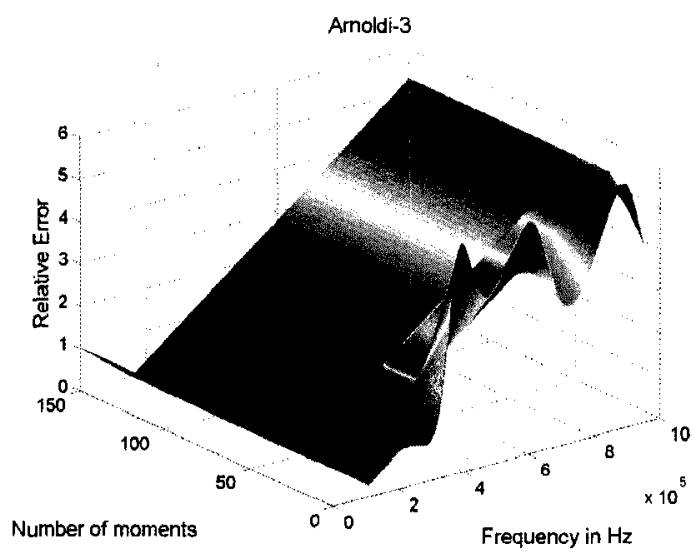

(c) Arnoldi-3

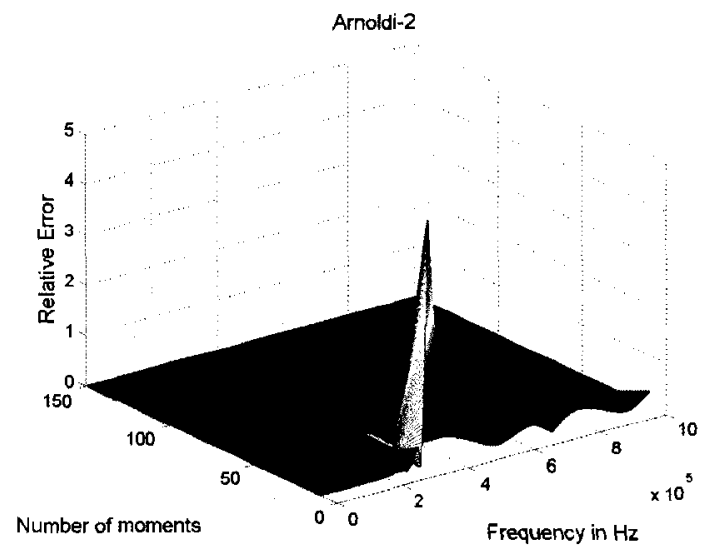

(b) Arnoldi-2

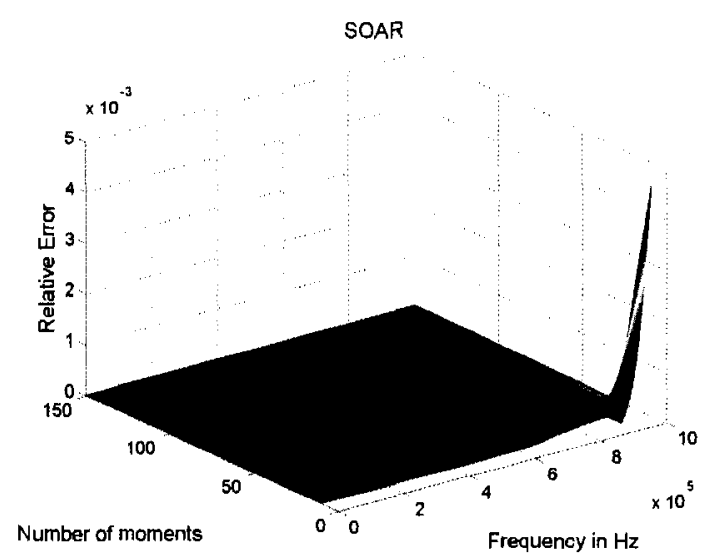

(d) SOAR

Figure C.8: Relative Error for the Frequency Response for 540-node cantilever, Damping=1.0 $\times$ $M$ 
Discussion: At a damping of $D=1.0 \times M$, the companion form of the Arnoldi algorithm that did not pass the stability tests did not match the frequency response of the full model. This was further evident in Figure C.8 where the magnitude of the relative error was on the order of $>10^{3}$ even at low frequencies. The other companion forms of the Arnoldi algorithm and SOAR all demonstrated some level of error but SOAR only exhibited poor matching at high frequencies. The relative error of the frequency response stayed low with lower moments for frequencies $\leq 2 \times 10^{5}$, and that at higher frequencies, moments $>50$ the relative error maintained a low order of magnitude.

\section{C.3.2 1350-node Cantilever}

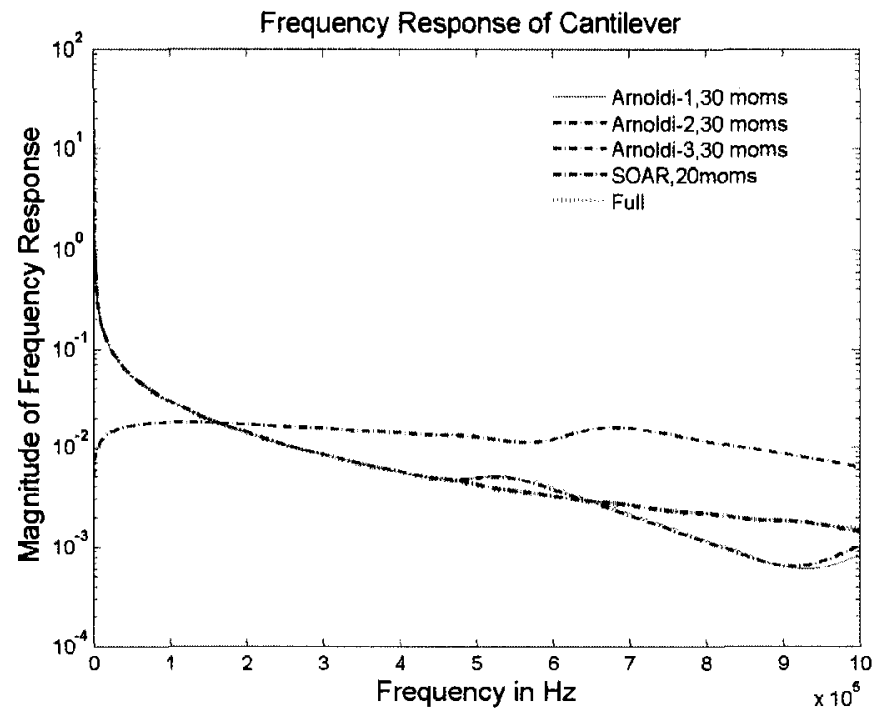

(a) Arnoldi-1, Arnoldi-2, Arnoldi-3, SOAR

Figure C.9: Comparison of frequency response for the full system and reduced model of a 1350 node Cantilever with Damping $=1.0 \times M$ 


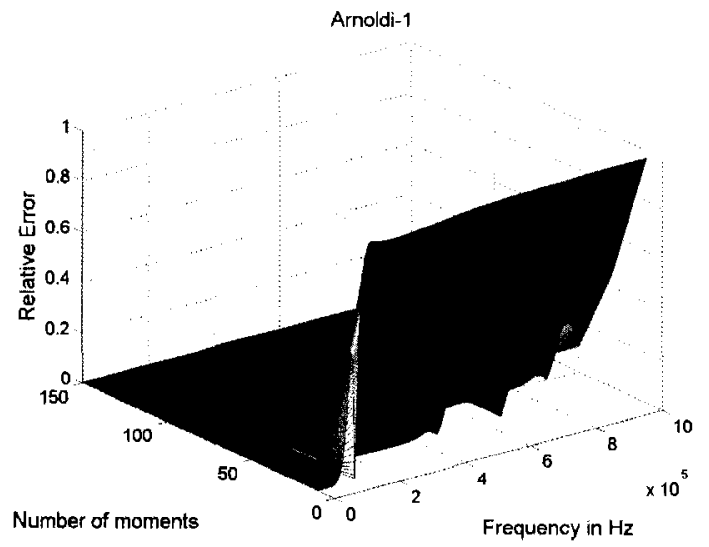

(a) Arnoldi-1

$$
\text { Arnoldi-3 }
$$

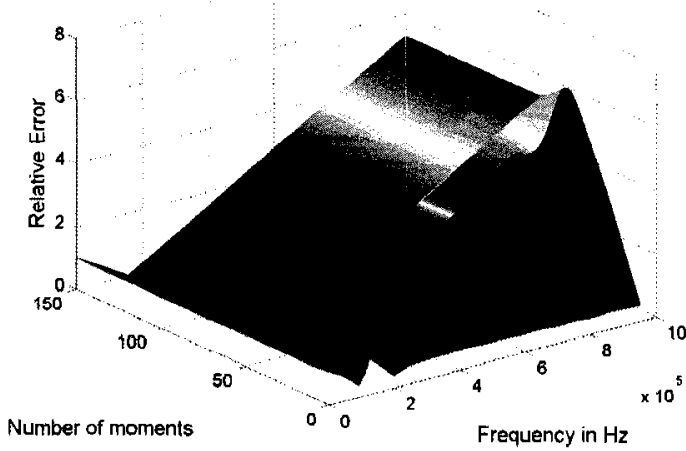

(c) Arnoldi-3

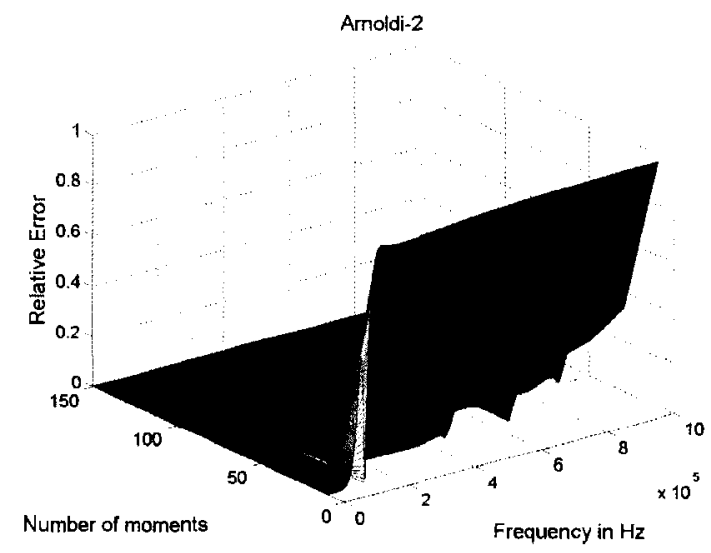

(b) Arnoldi-2

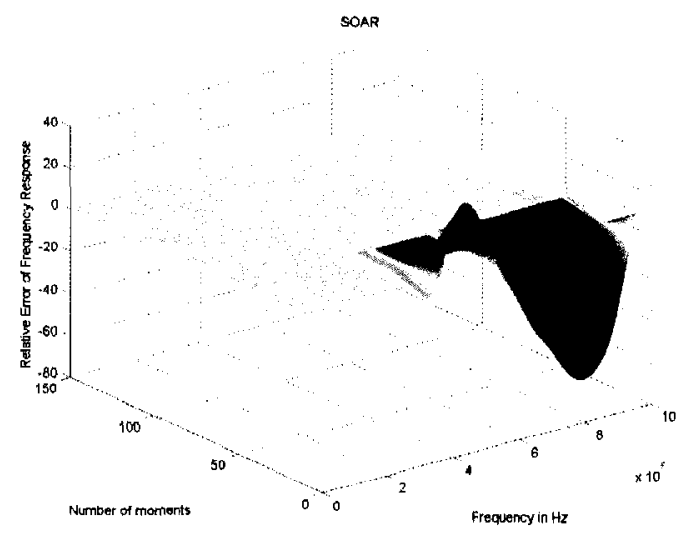

(d) SOAR

Figure C.10: Relative Error for the Frequency Response for 1350-node cantilever,Damping= $1.0 \times M$ 
Discussion: The frequency response for the 1350-node cantilever exhibited a high amount of errors at low frequencies for smaller number of moments. Moments $<30$, demonstrated a high amount of relative error for both stable forms of Arnoldi and SOAR as shown in Figure C.10. The companion form of Arnoldi that failed the stability tests demonstrated a higher level of error at all frequencies.

\section{C.3.3 2700-node Cantilever}

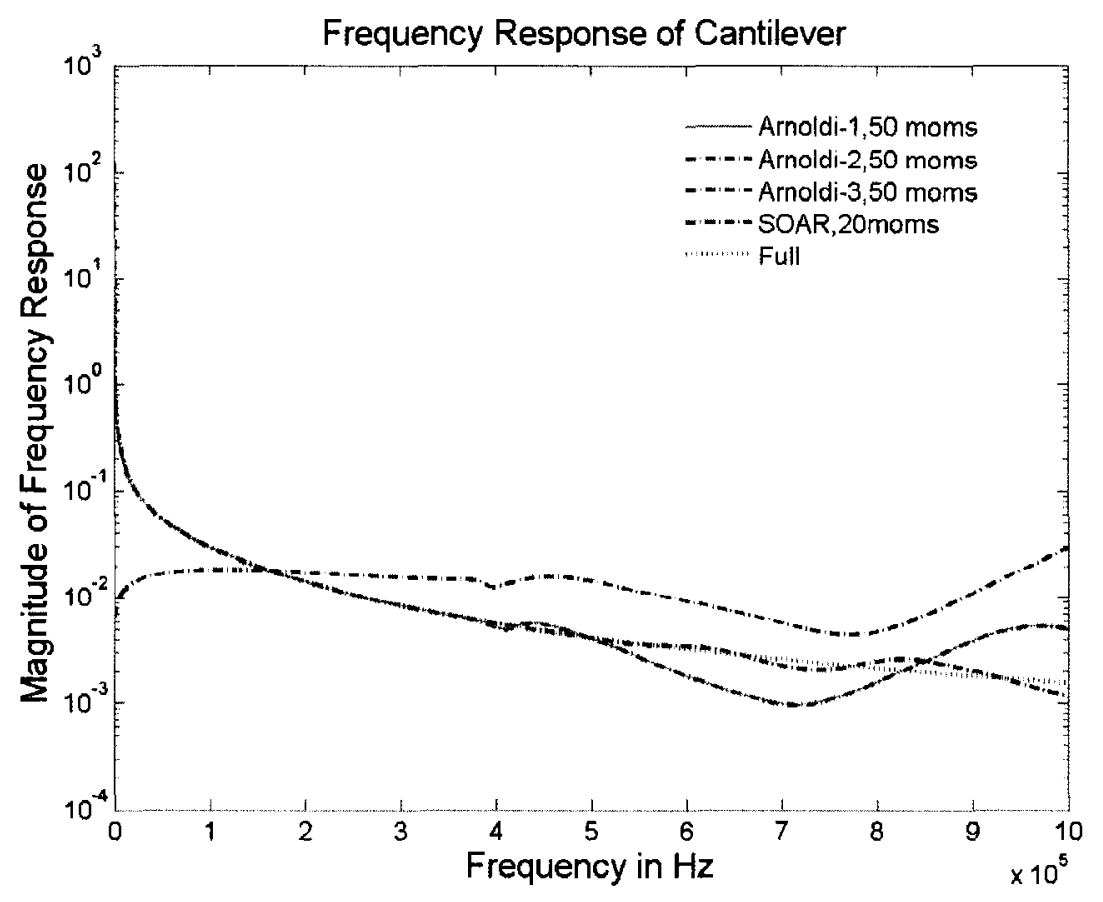

(a) Arnoldi-1, Arnoldi-2, Arnoldi-3, SOAR

Figure C.11: Comparison of frequency response for the full system and reduced model of a 2700 node Cantilever with Damping $=1.0 \times M$ 


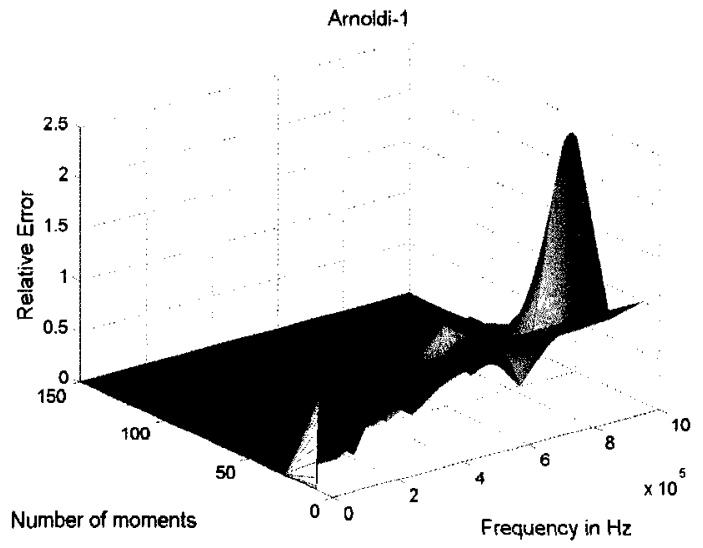

(a) Arnoldi-1

Arnoldi-3

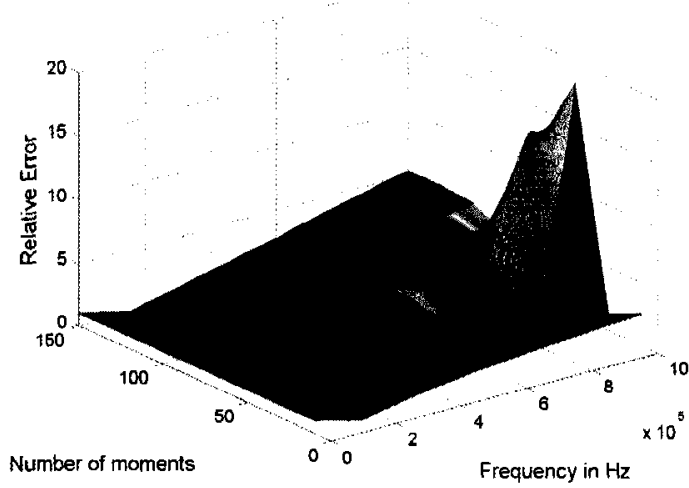

(c) Arnoldi-3

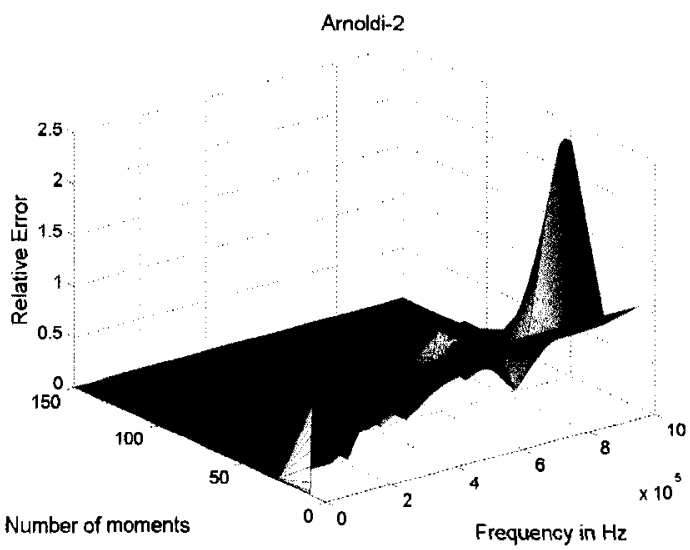

(b) Arnoldi-2 SOAR

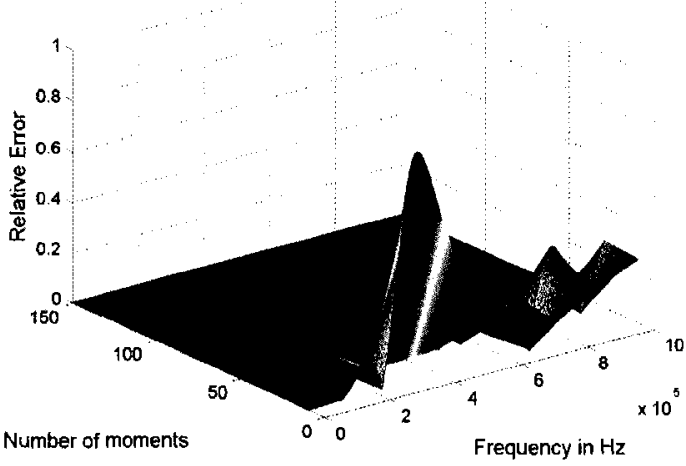

(d) SOAR

Figure C.12: Relative Error for the Frequency Response for 2700-node cantilever, Damping= $1.0 \times M$ 
Discussion: The frequency response for the 2700-node cantilever exhibited a high amount of errors at low frequencies for smaller number of moments. There was no clear trend to determine the minimum number of moments that would provide a match with low relative error at a specific frequency cut-off point, as shown in Figure C.12. Moments $<30$ are clearly provided too much error, while moments $>30$ had low relative errors up to frequencies of $10^{6}$.

\section{C.4 Cantilever Models with Damping $=10^{-3} \times M$}

\section{0-node Cantilever}

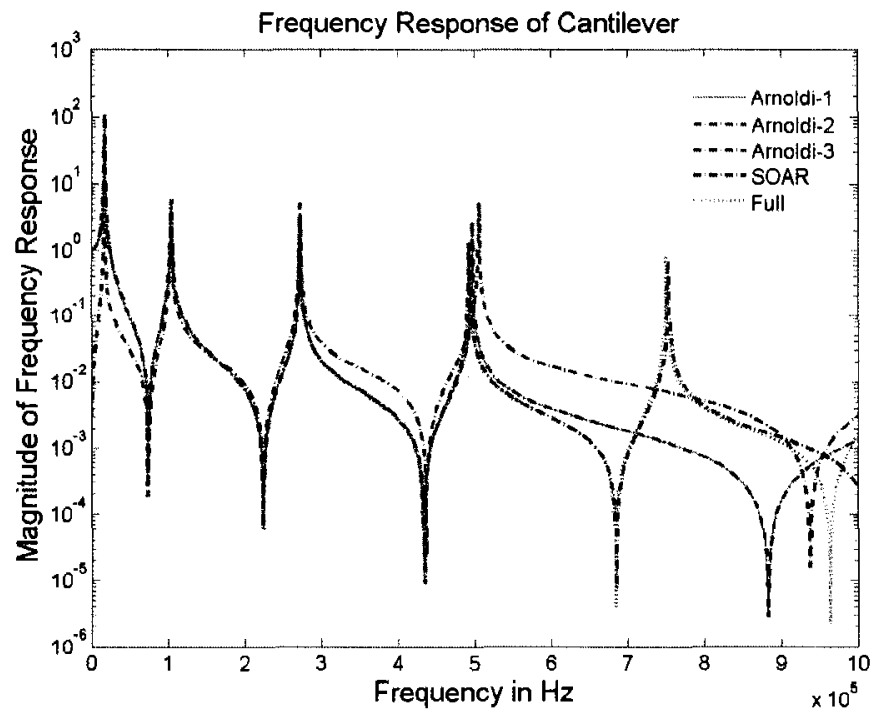

(a) Arnoldi-1, Arnoldi-2, Arnoldi-3, SOAR

Figure C.13: Comparison of frequency responses for the full system and reduced model of a 540-node Cantilever with Damping $=10^{-3} \times M$ 


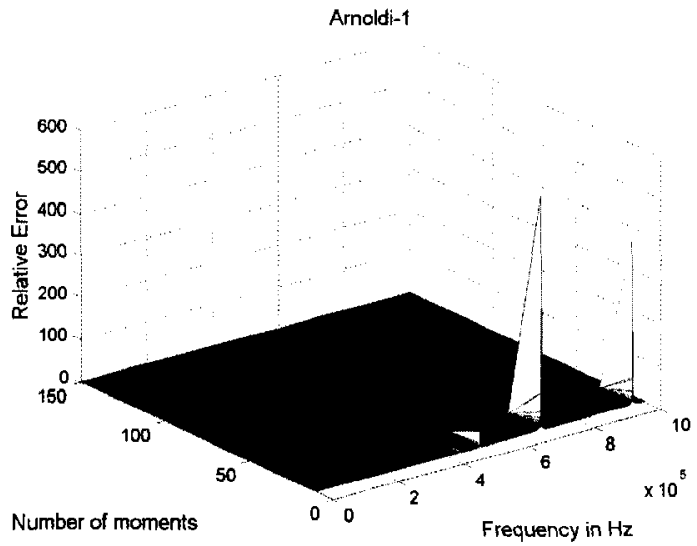

(a) Arnoldi-1

Arnoldi-3

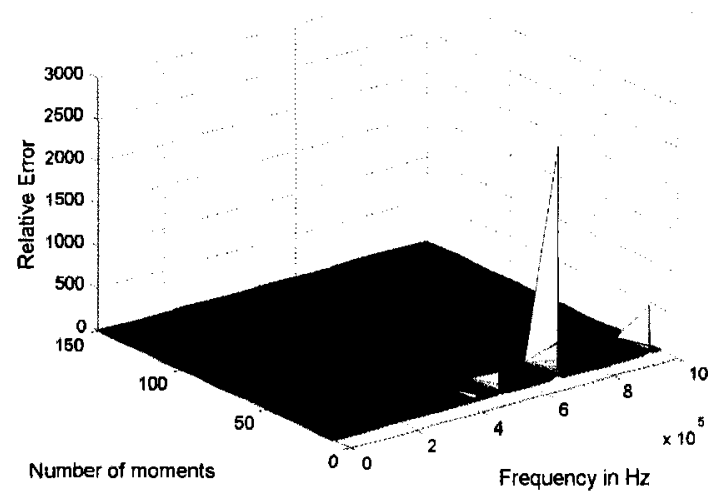

(c) Arnoldi-3

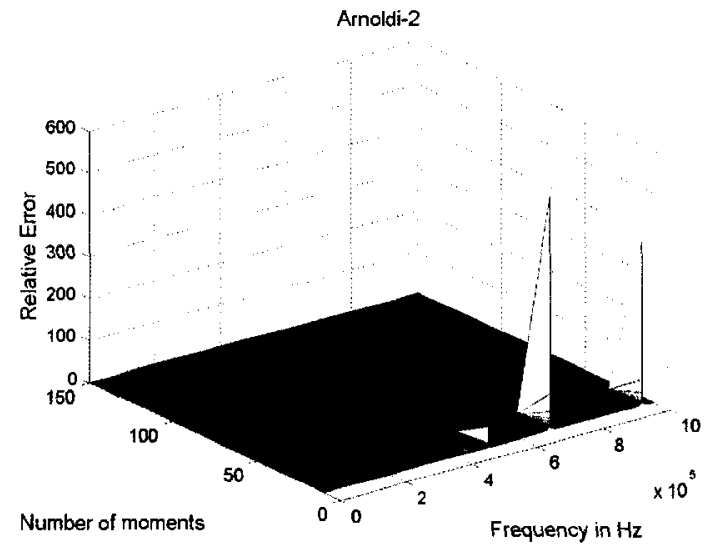

(b) Arnoldi-2

SOAR

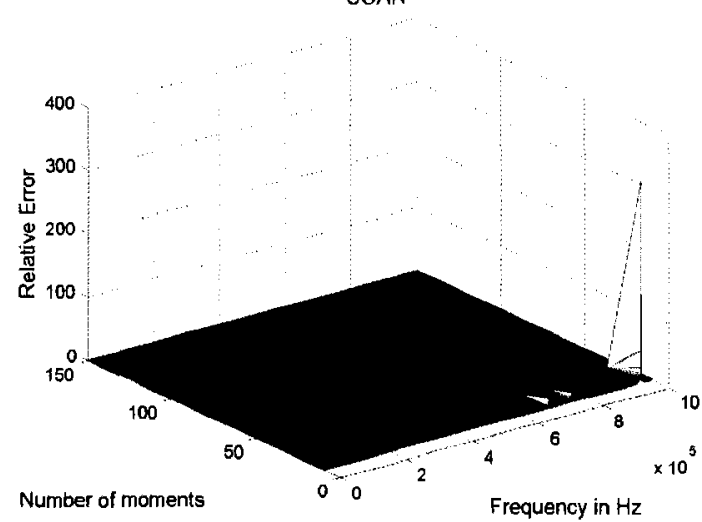

(d) SOAR

Figure C.14: Relative Error for the Frequency Response for 540-node cantilever, Damping= $10^{-3} \times M$ 


\section{C.4.1 1350-node Cantilever}

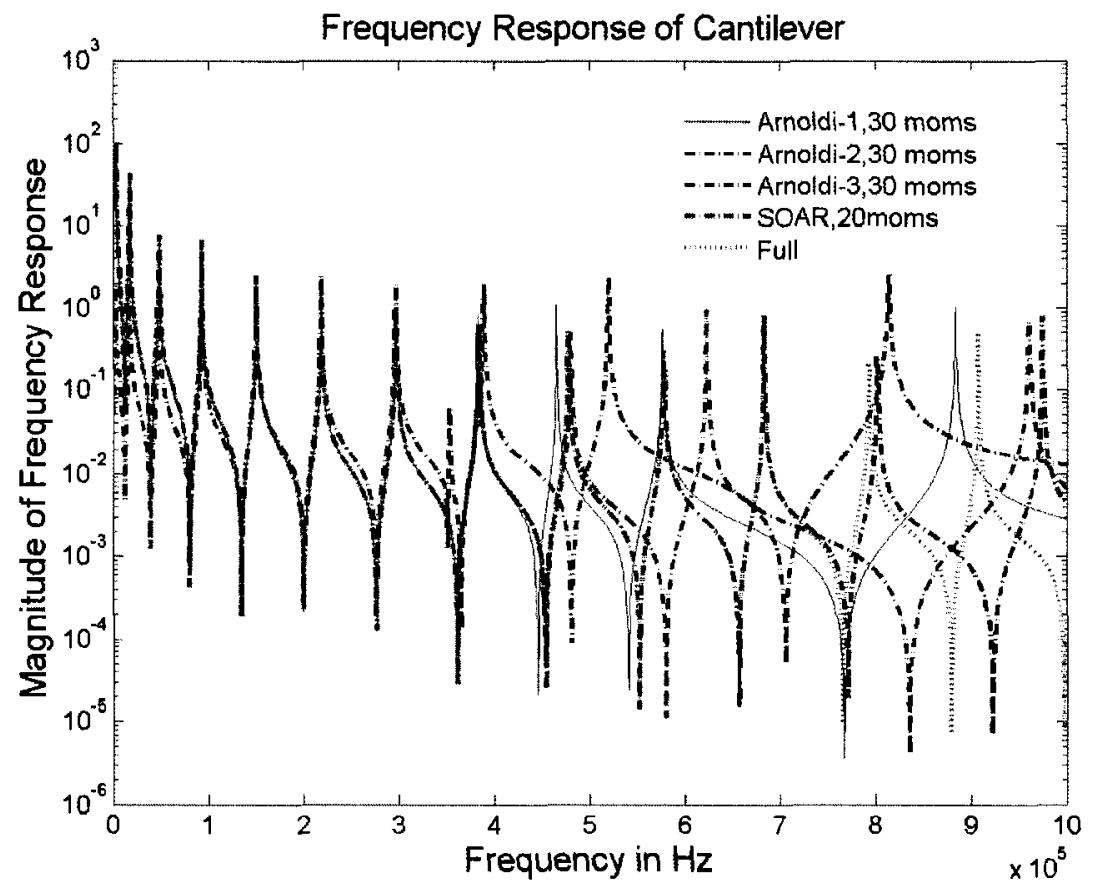

(a) Arnoldi-1, Arnoldi-2, Arnoldi-3, SOAR

Figure C.15: Comparison of frequency responses for the full system and reduced model of a 1350 -node Cantilever with Damping $=10^{-3} \times M$ 


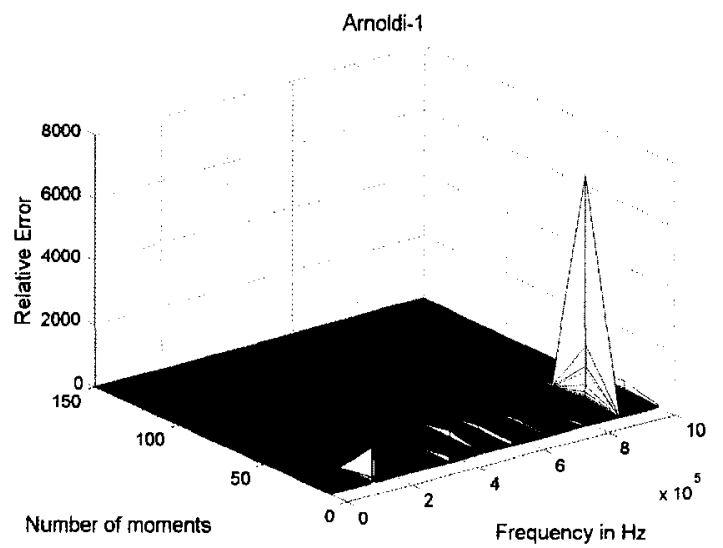

(a) Arnoldi-1

Arnoldi-3

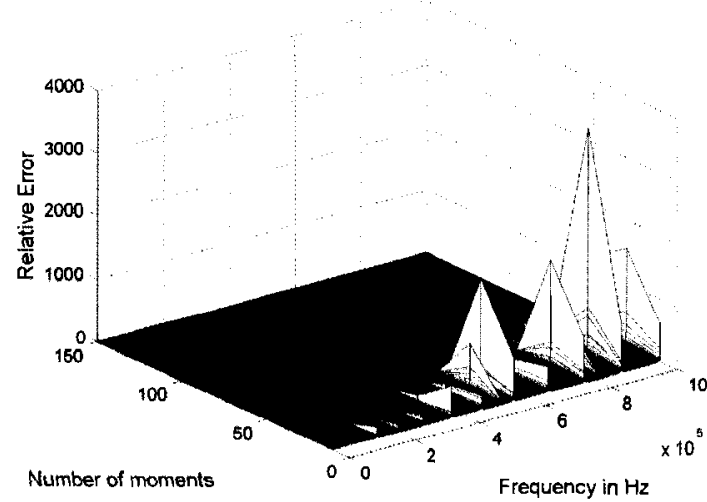

(c) Arnoldi-3

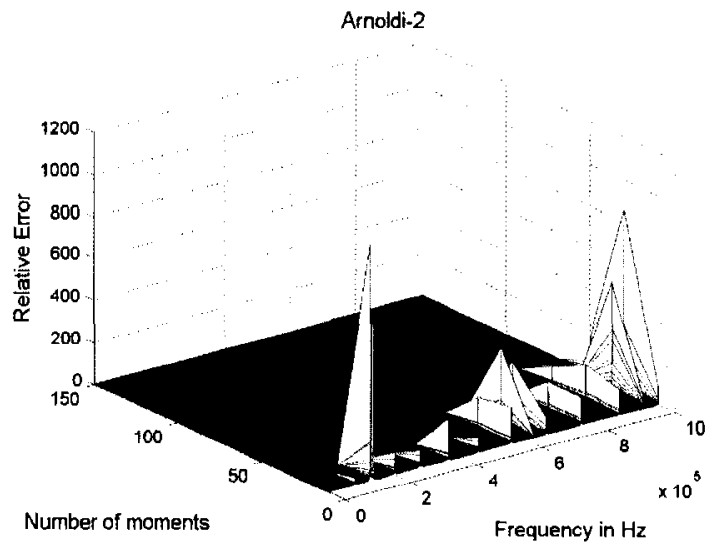

(b) Arnoldi-2

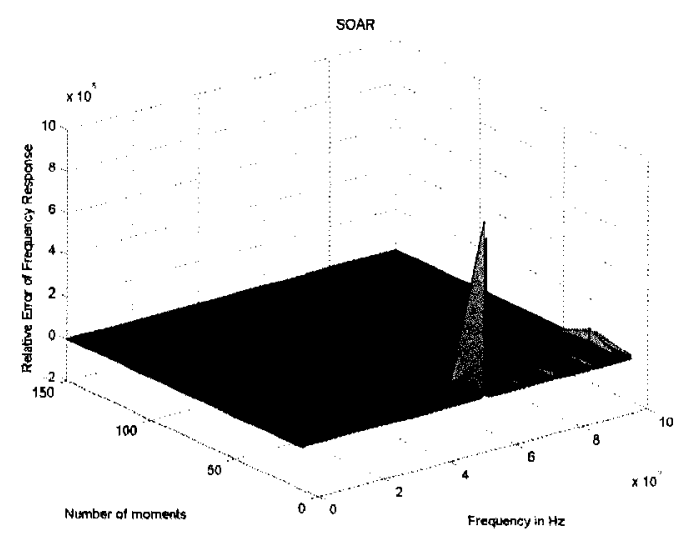

(d) SOAR

Figure C.16: Relative Error for the Frequency Response for 1350-node cantilever,Damping= $10^{-3} \times M$ 


\section{C.4.2 2700-node Cantilever}

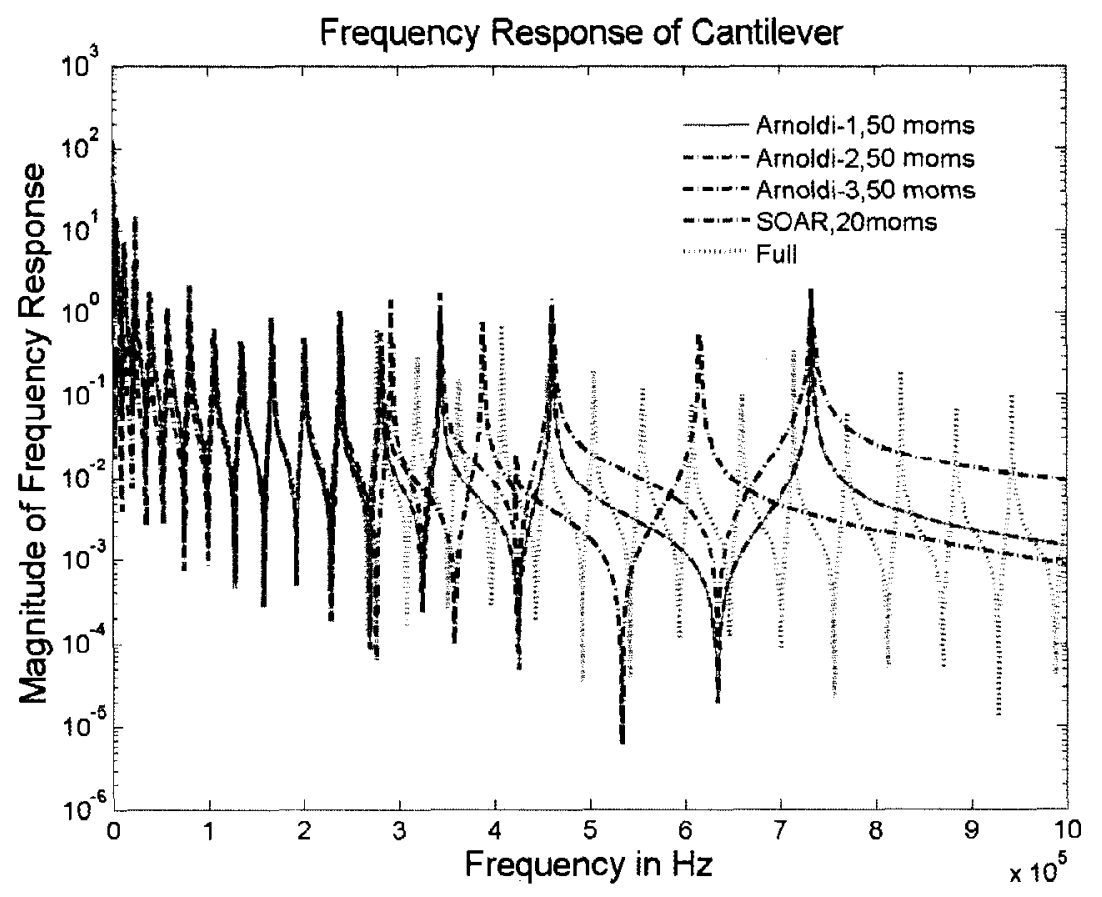

(a) Arnoldi-1, Arnoldi-2, Arnoldi-3, SOAR

Figure C.17: Comparison of frequency responses for the full system and reduced model of a 2700-node Cantilever with Damping $=10^{-3} \times M$ 


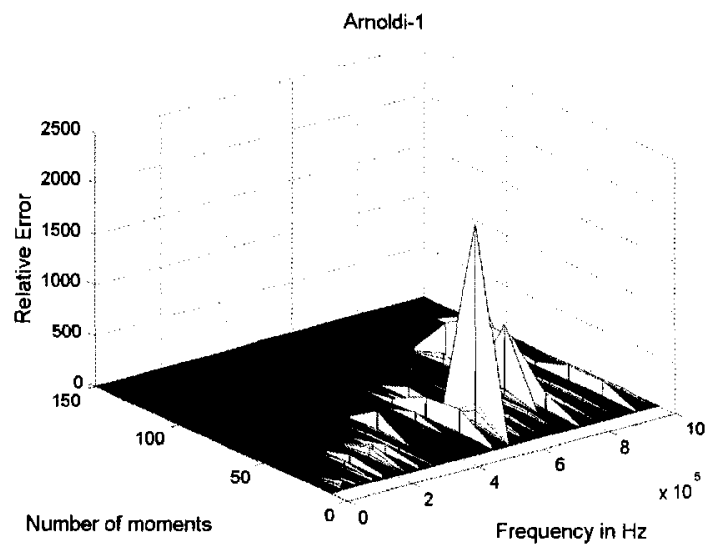

(a) Arnoldi-1

Arnoldi-3

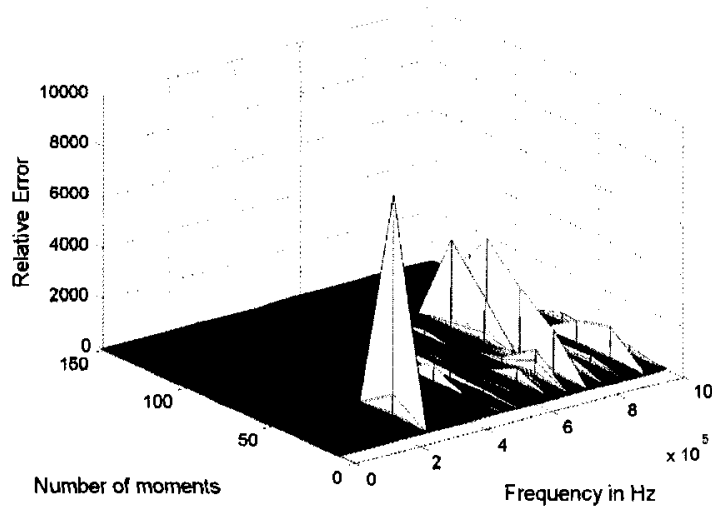

(c) Arnoldi-3

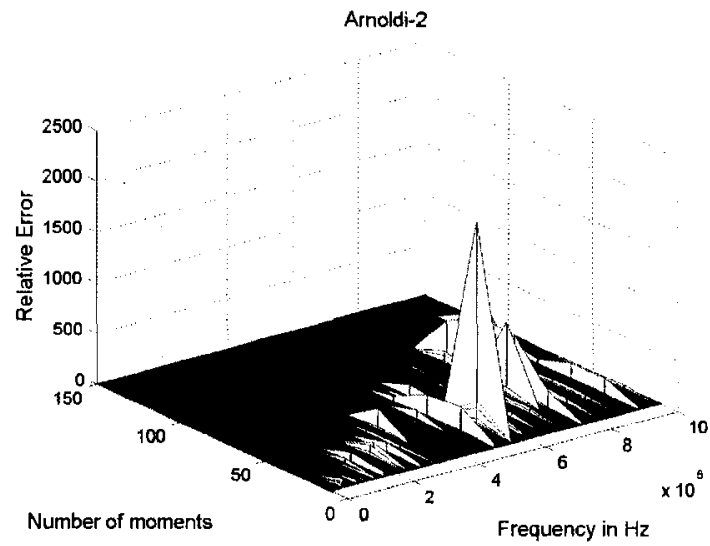

(b) Arnoldi-2

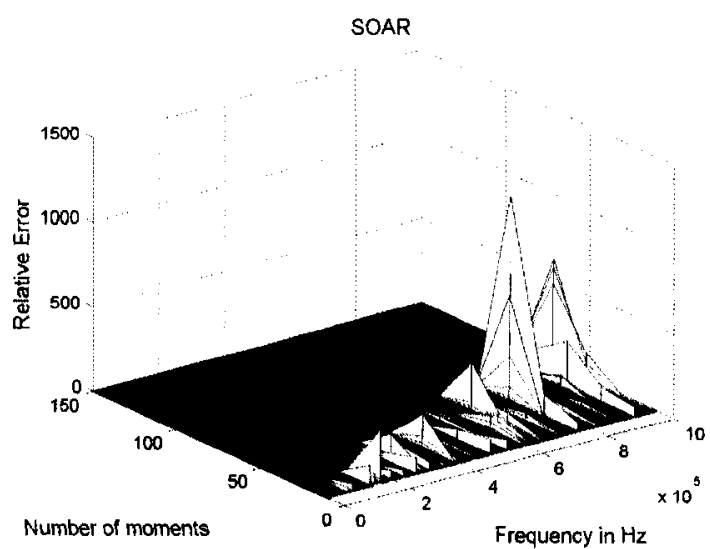

(d) SOAR

Figure C.18: Relative Error for the Frequency Response for 2700-node cantilever, Damping= $10^{-3} \times M$ 


\section{C.5 Cantilever Models with Damping $=10^{-4} \times M$}

\section{0-node Cantilever}

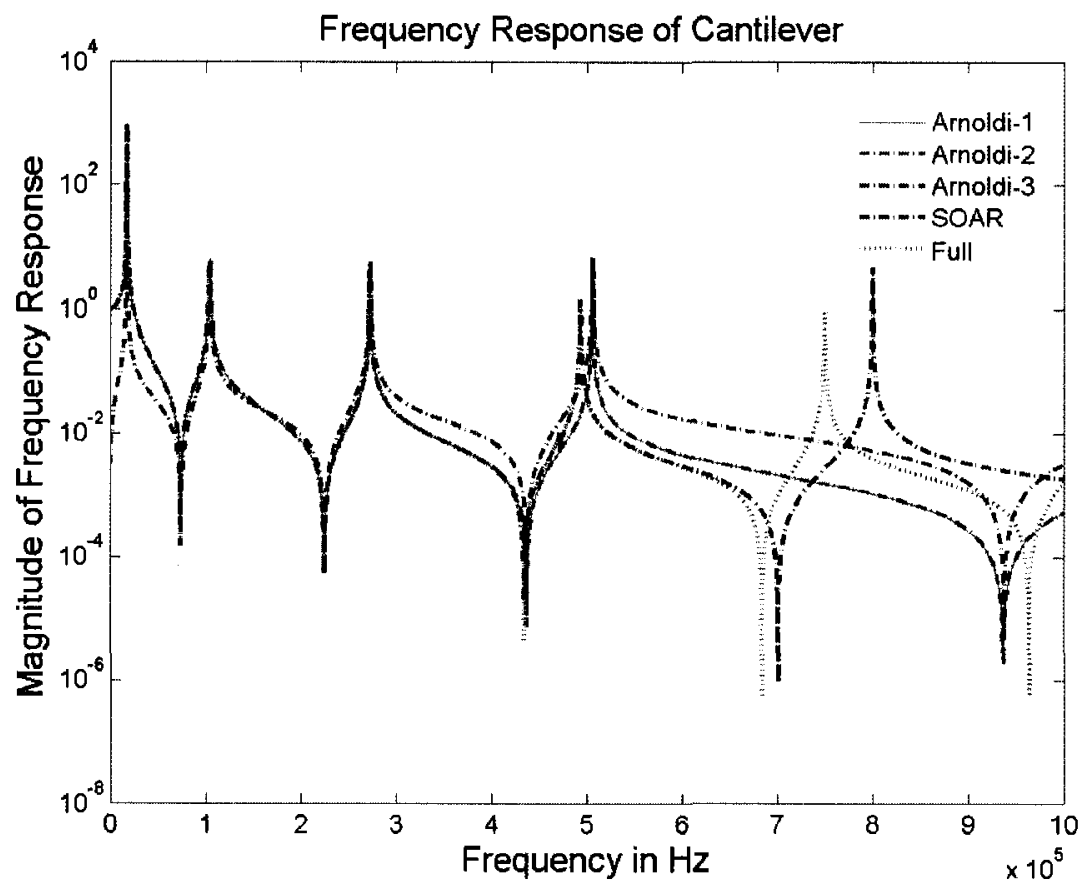

(a) Arnoldi-1, Arnoldi-2, Arnoldi-3, SOAR

Figure C.19: Comparison of frequency responses for the full system and reduced model of a 540-node Cantilever with Damping $=10^{-4} \times M$ 


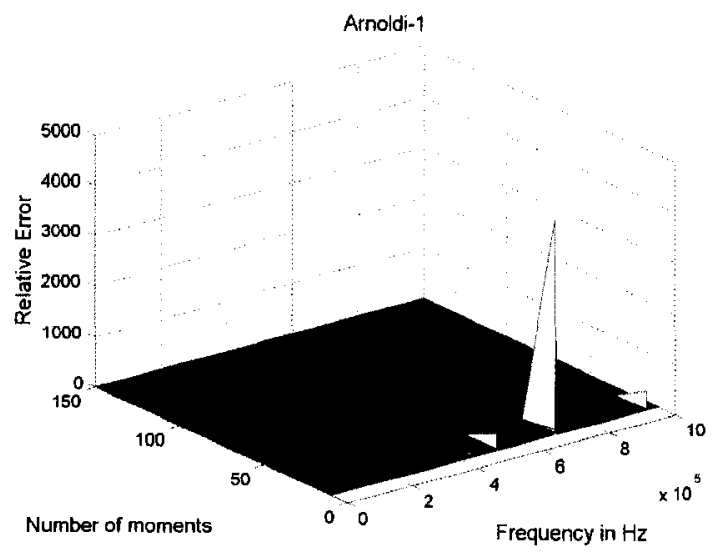

(a) Arnoldi-1

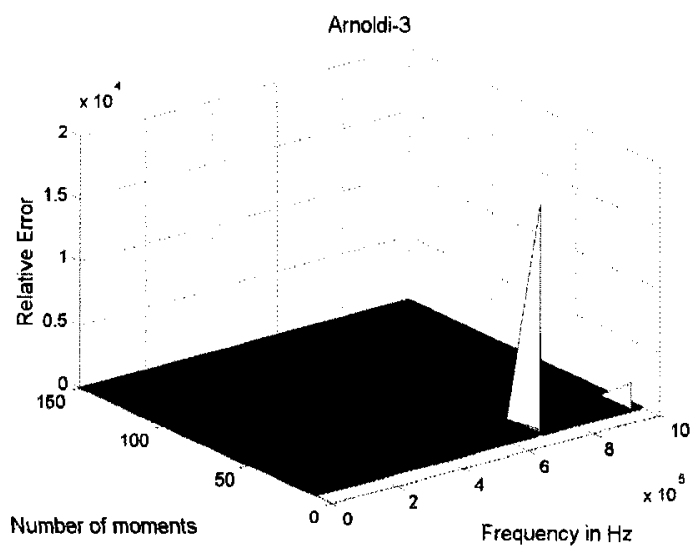

(c) Arnoldi-3

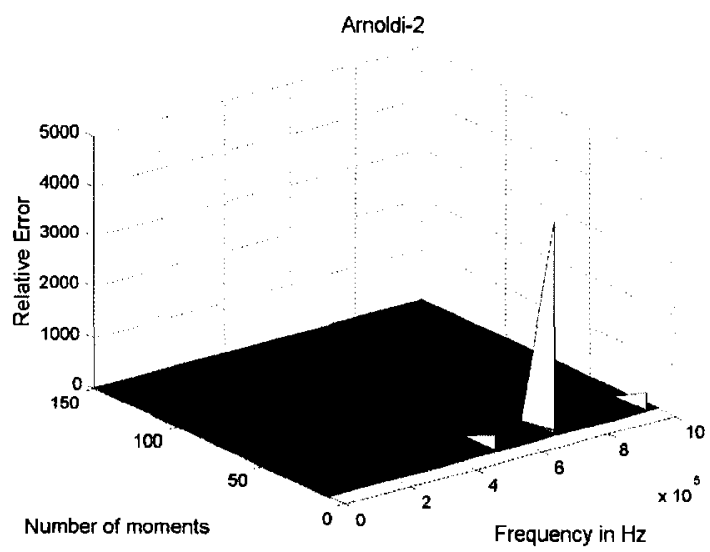

(b) Arnoldi-2

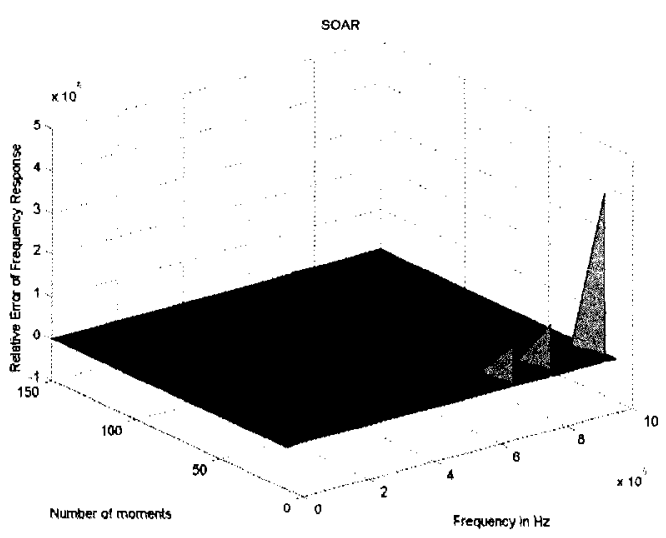

(d) SOAR

Figure C.20: Relative Error for the Frequency Response for 540-node cantilever, Damping= $10^{-4} \times M$ 


\section{C.5.1 1350-node Cantilever}

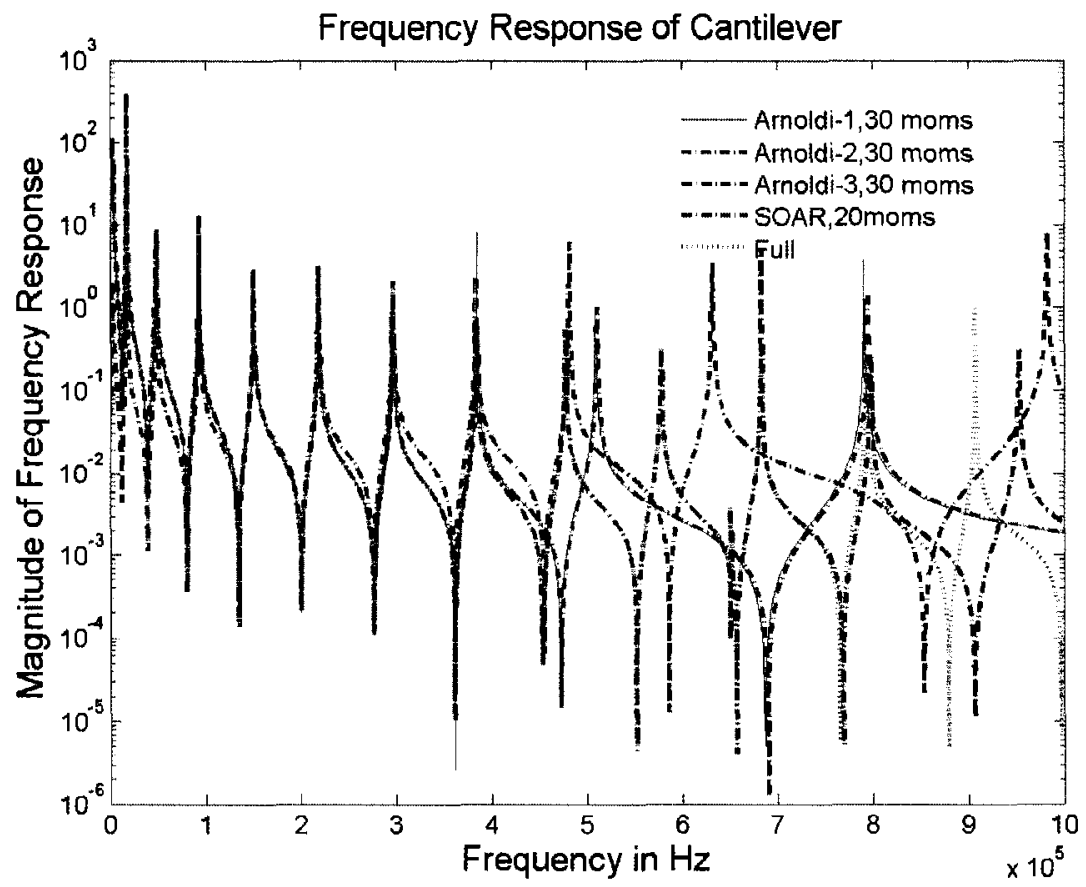

(a) Arnoldi-1, Arnoldi-2, Arnoldi-3, SOAR

Figure C.21: Comparison of frequency responses for the full system and reduced model of a 1350-node Cantilever with Damping $=10^{-4} \times M$ 


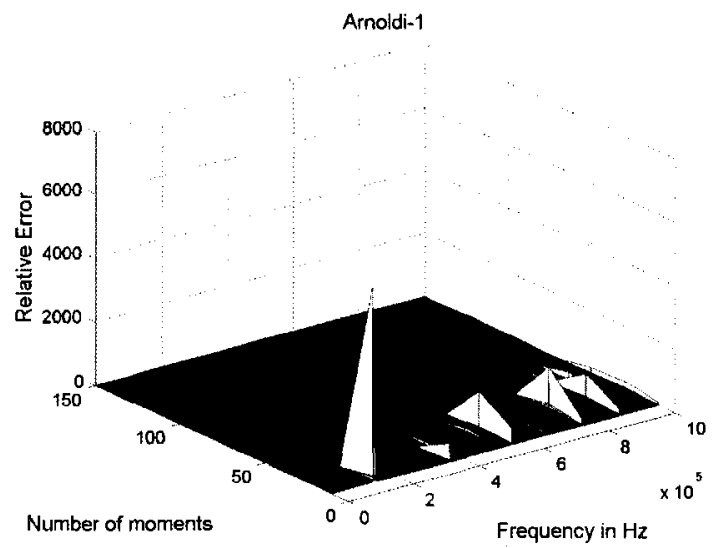

(a) Arnoldi-1

Arnoldi-3

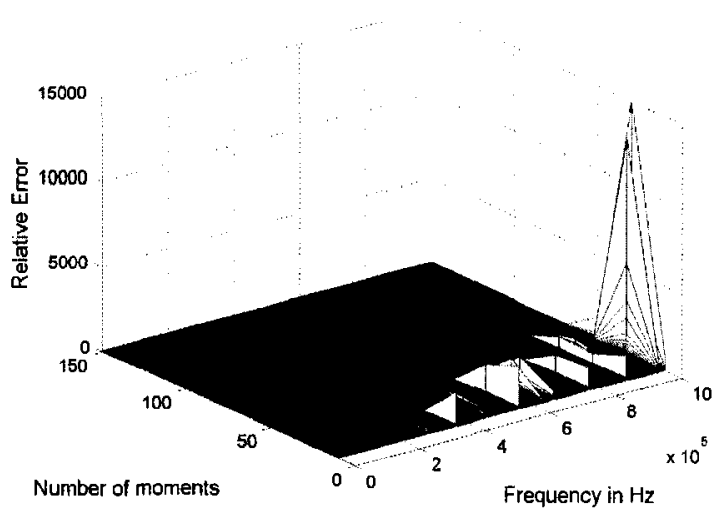

(c) Arnoldi-3

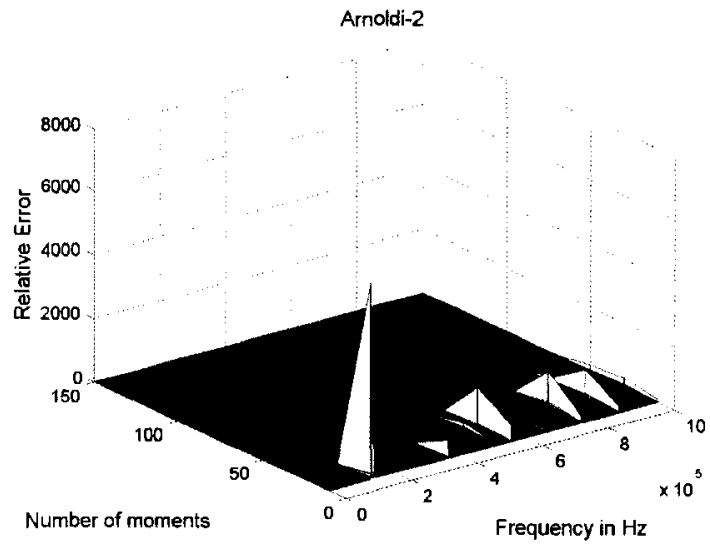

(b) Arnoldi-2

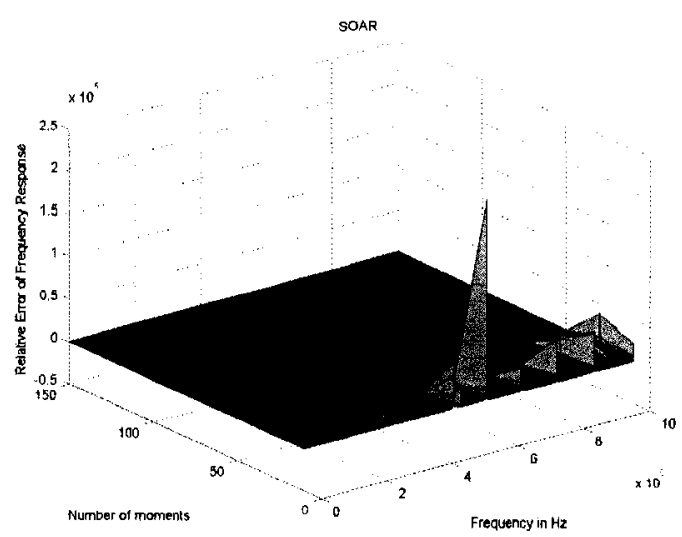

(d) SOAR

Figure C.22: Relative Error for the Frequency Response for 1350-node cantilever,Damping= $10^{-4} \times M$ 


\section{C.5.2 2700-node Cantilever}

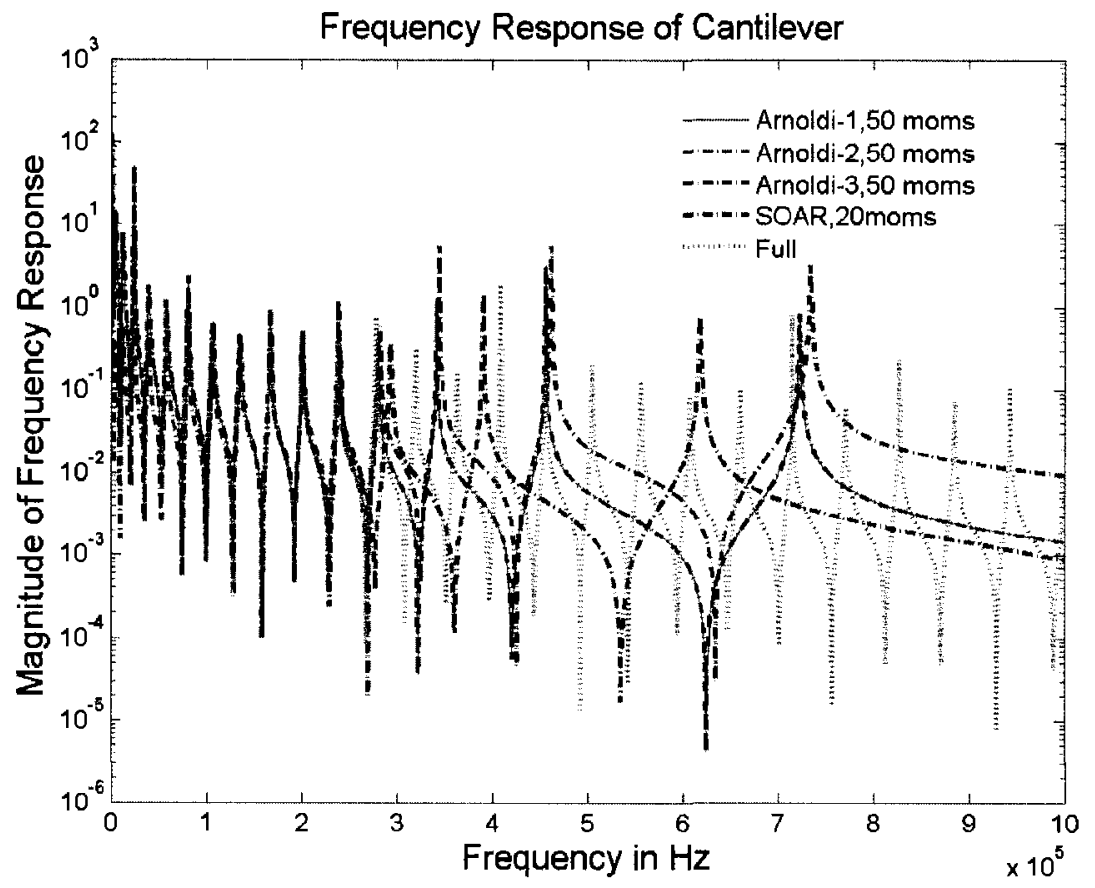

(a) Arnoldi-1, Arnoldi-2, Arnoldi-3, SOAR

Figure C.23: Comparison of frequency responses for the full system and reduced model of a 2700-node Cantilever with Damping $=10^{-4} \times M$ 


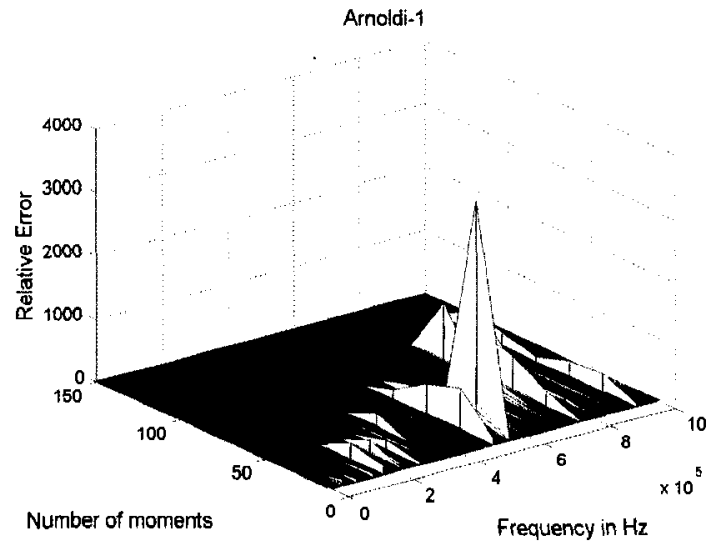

(a) Arnoldi-1

Arnoldi-3

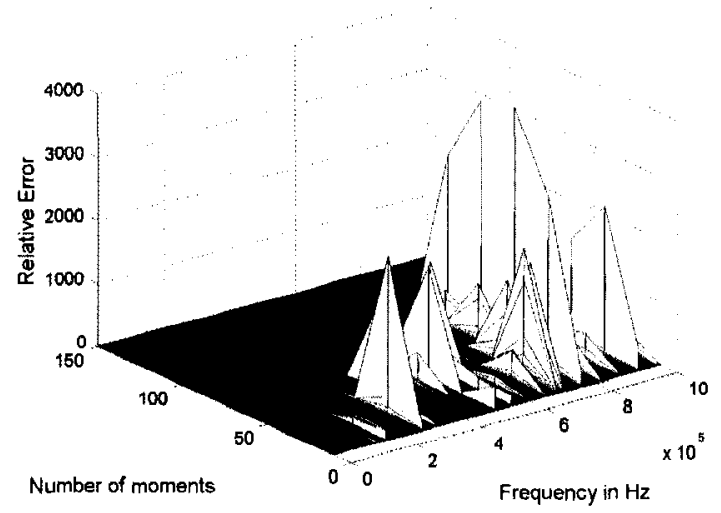

(c) Arnoldi-3

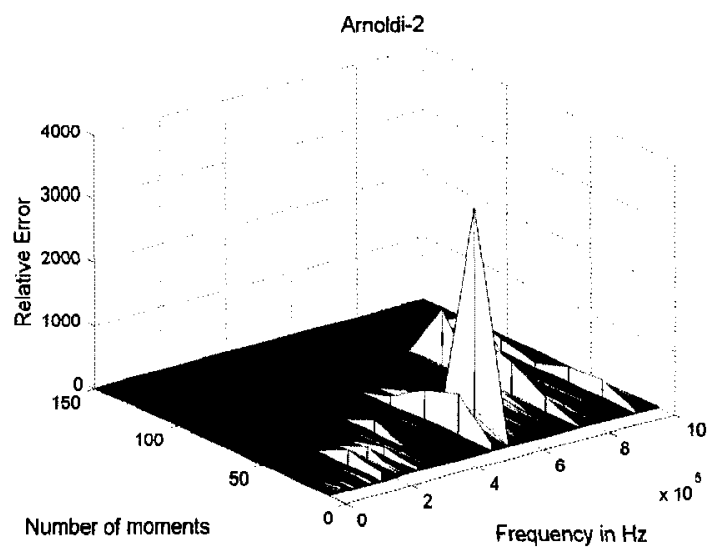

(b) Arnoldi-2

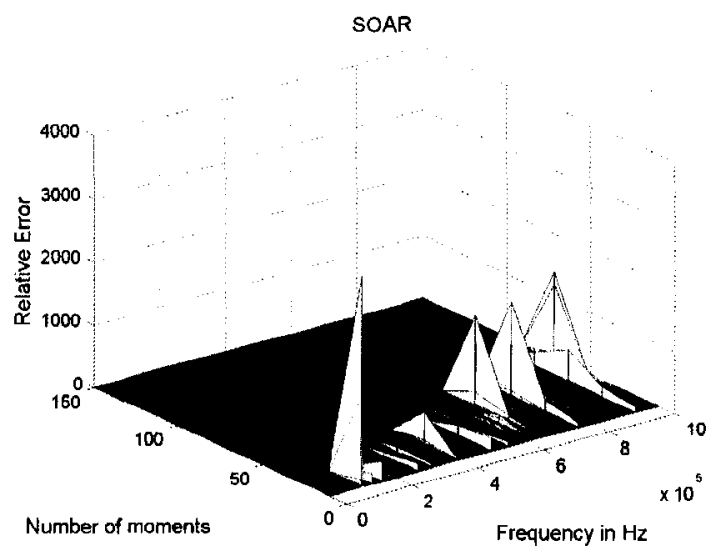

(d) SOAR

Figure C.24: Relative Error for the Frequency Response for 2700-node cantilever, Damping= $10^{-4} \times M$ 


\section{C.6 Cantilever Models with Damping $=10^{-5} \times M$}

\section{C.6.1 540-node Cantilever}

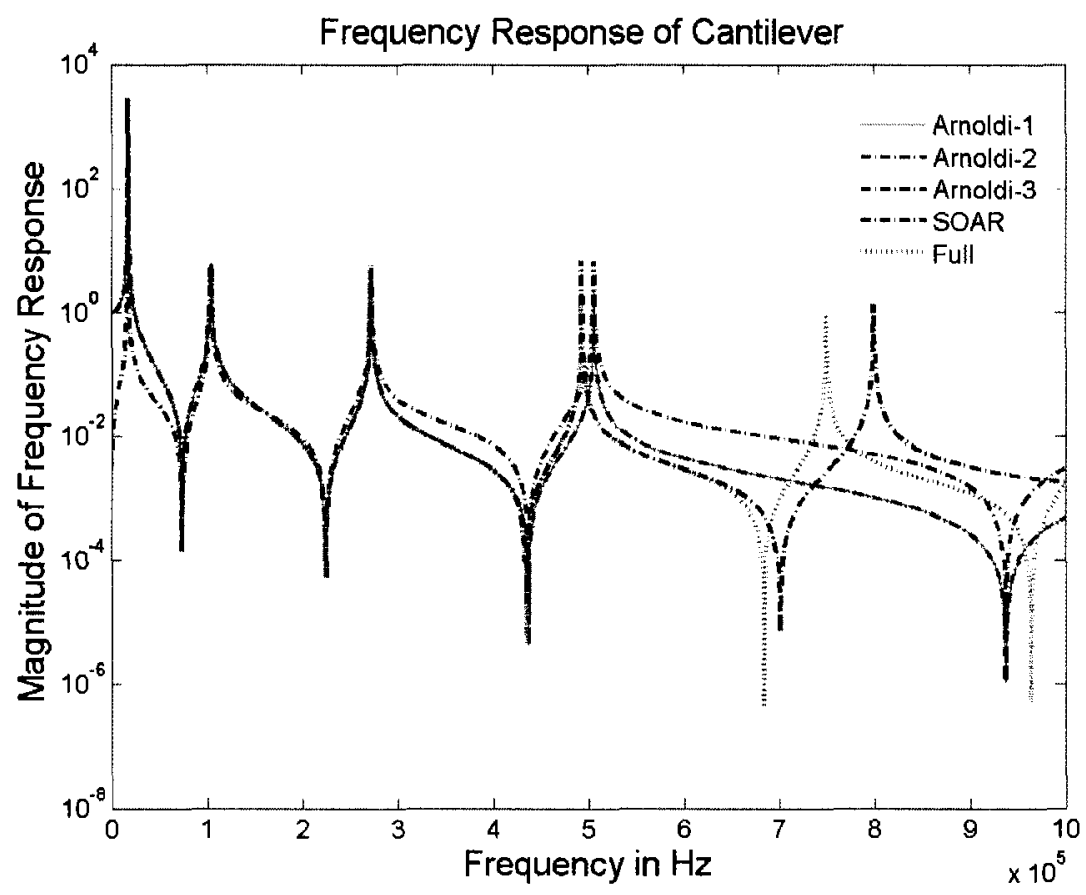

(a) Arnoldi-1, Arnoldi-2, Arnoldi-3, SOAR

Figure C.25: Comparison of frequency responses for the full system and reduced model of a 540-node Cantilever with Damping $=10^{-5} \times M$ 


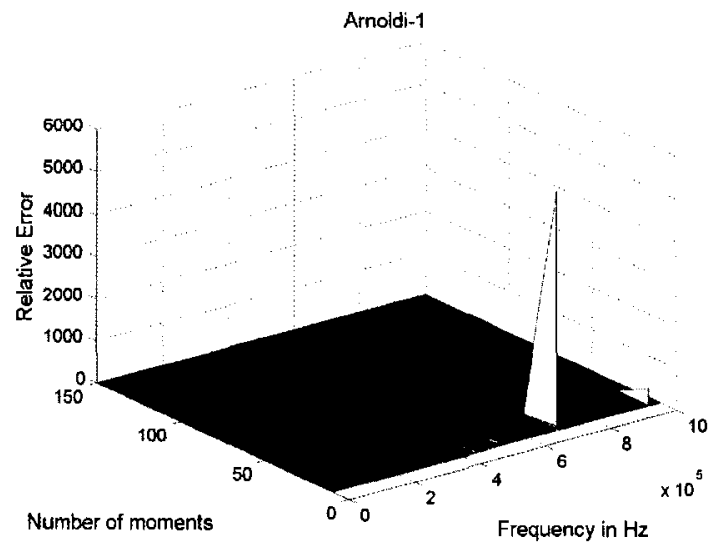

(a) Arnoldi-1

Arnoldi-3

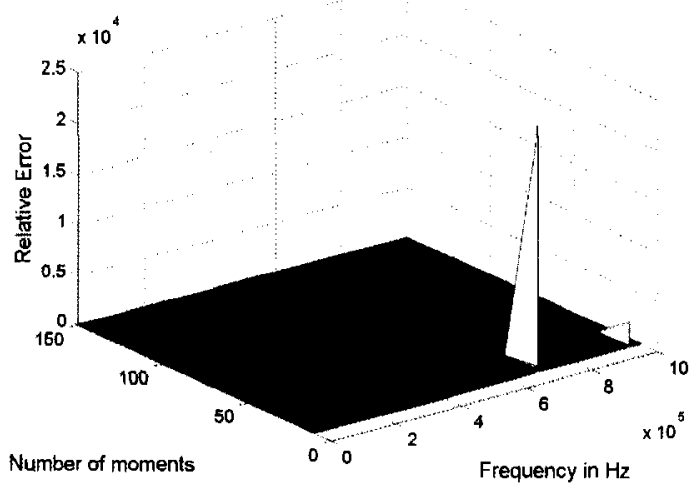

(c) Arnoldi-3

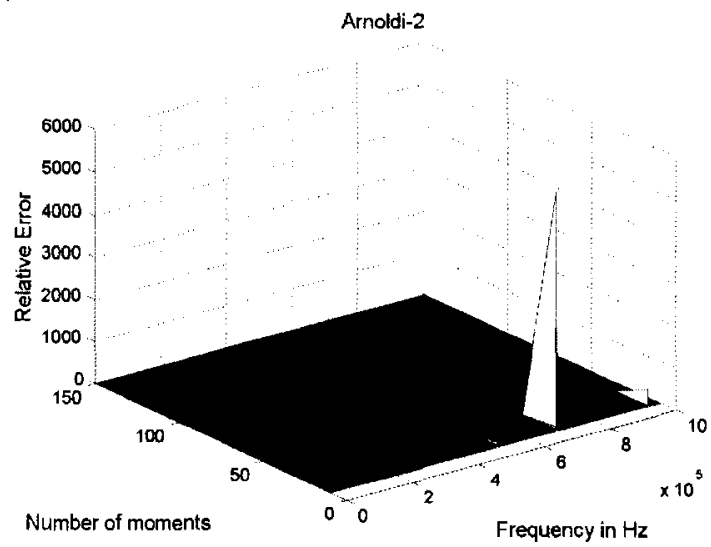

(b) Arnoldi-2

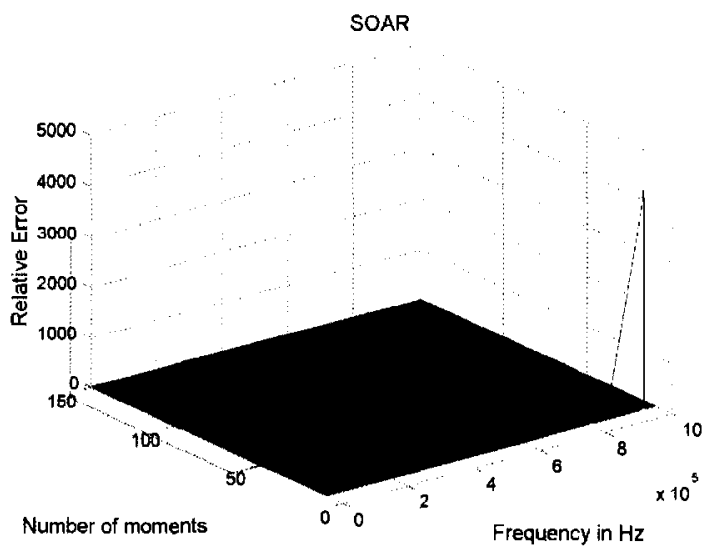

(d) SOAR

Figure C.26: Relative Error for the Frequency Response for 540-node cantilever, Damping= $10^{-5} \times M$ 


\section{C.6.2 1350-node Cantilever}

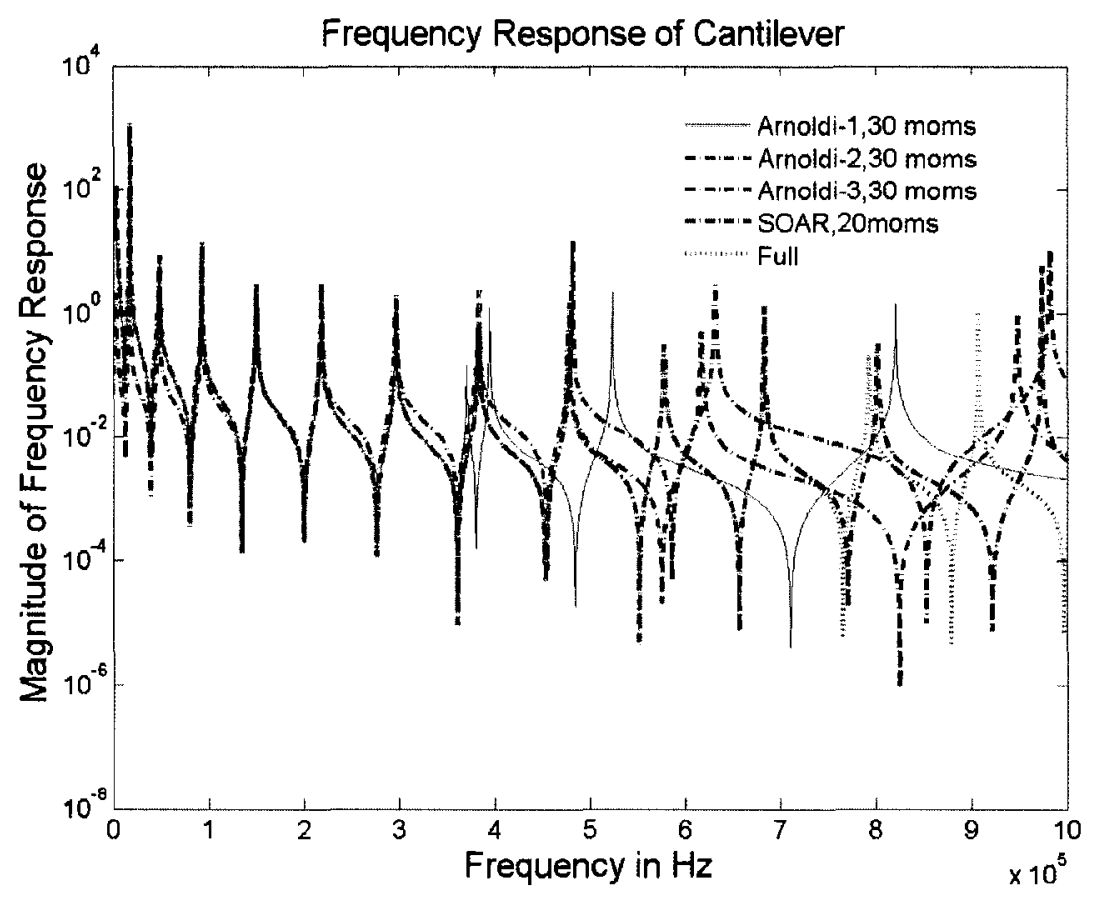

(a) Arnoldi-1, Arnoldi-2, Arnoldi-3, SOAR

Figure C.27: Comparison of frequency responses for the full system and reduced model of a 1350-node Cantilever with Damping $=10^{-5} \times M$ 


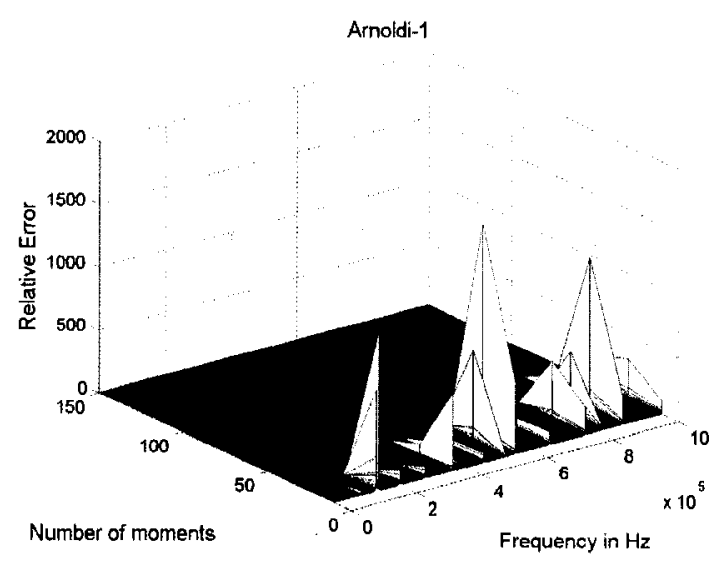

(a) Arnoldi-1

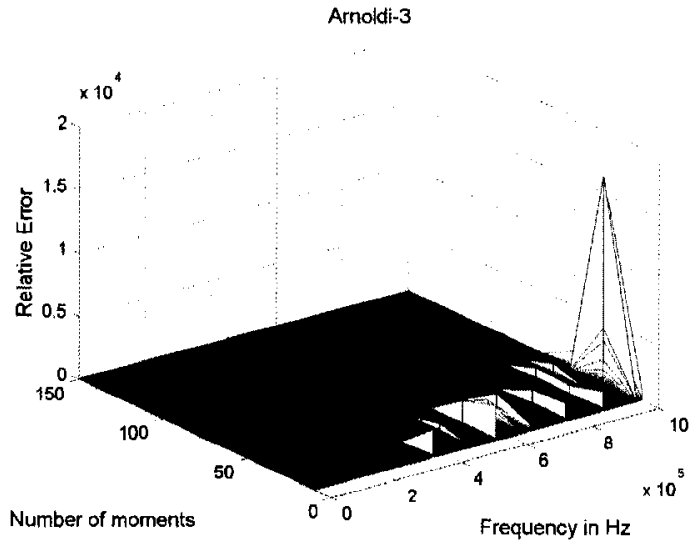

(c) Arnoldi-3

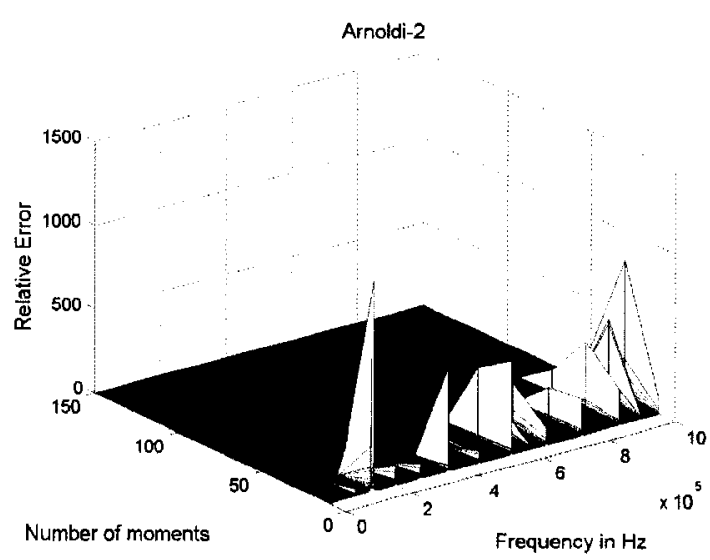

(b) Arnoldi-2

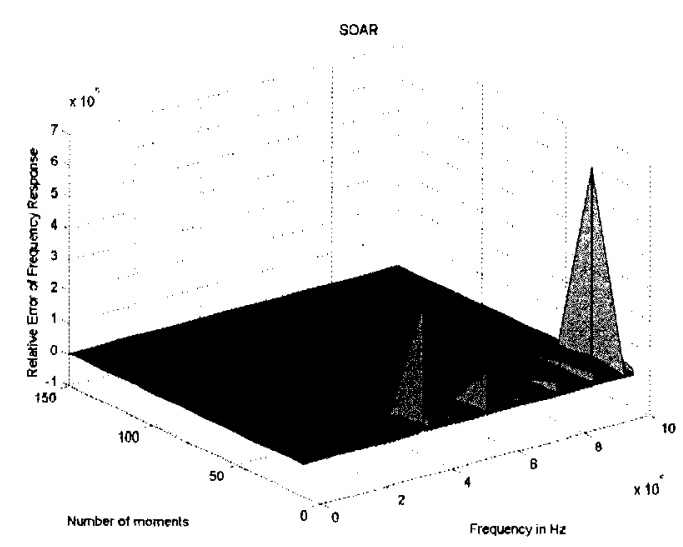

(d) SOAR

Figure C.28: Relative Error for the Frequency Response for 1350-node cantilever,Damping $=10^{-5} \times M$ 


\section{C.6.3 2700-node Cantilever}

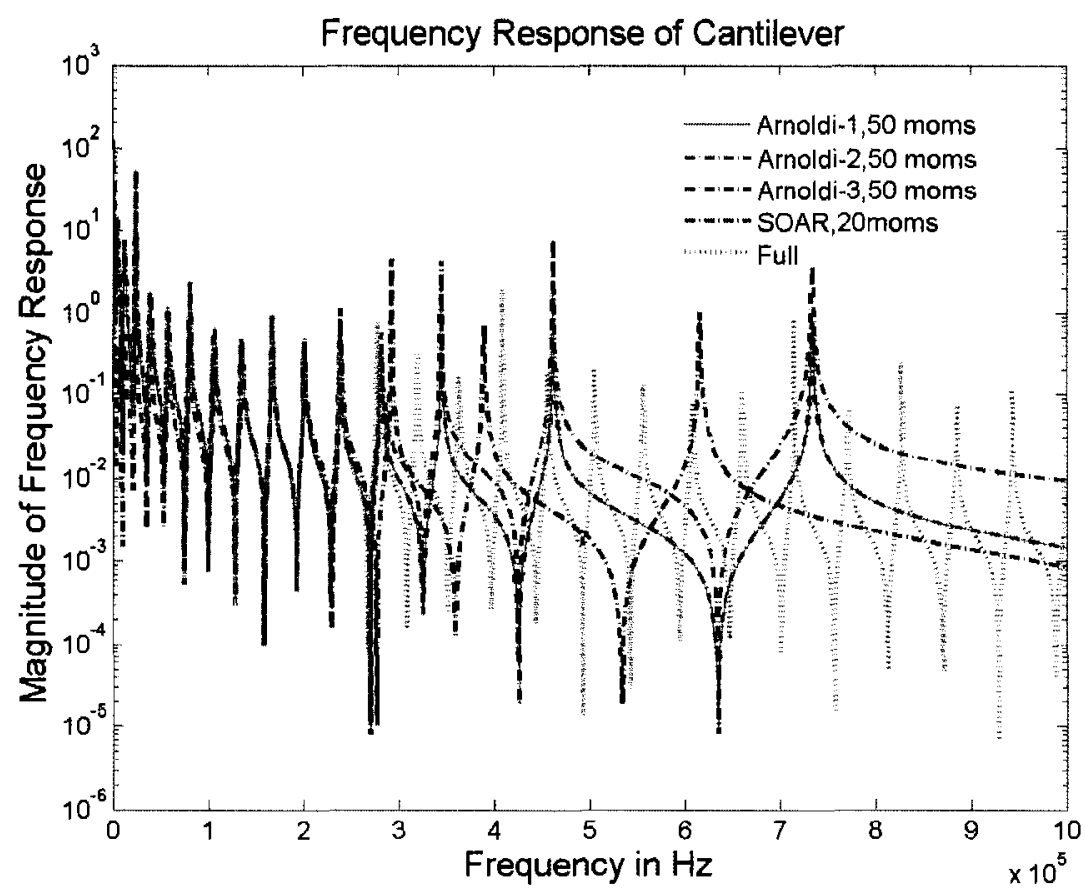

(a) Arnoldi-1, Arnoldi-2, Arnoldi-3, SOAR

Figure C.29: Comparison of frequency responses for the full system and reduced model of a 2700-node Cantilever with Damping $=10^{-5} \times M$ 


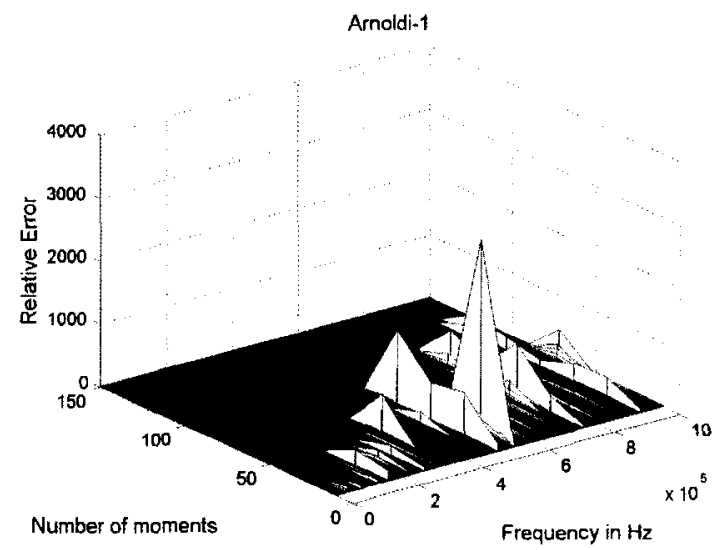

(a) Arnoldi-1

Arnoldi-3

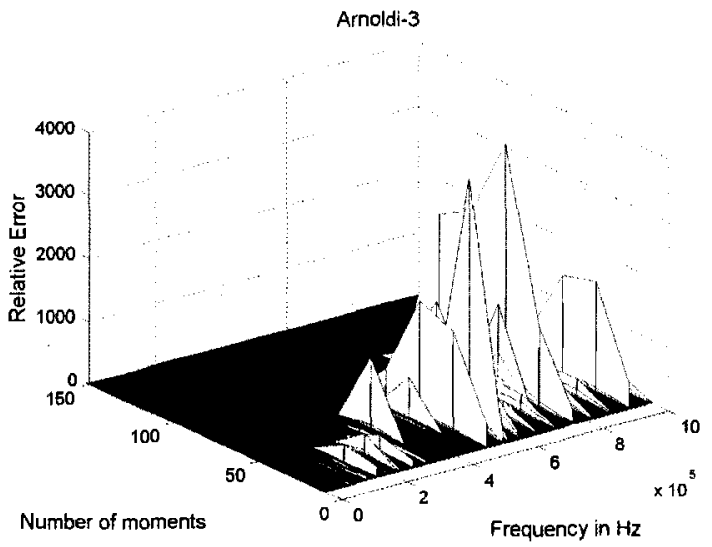

(c) Arnoldi-3

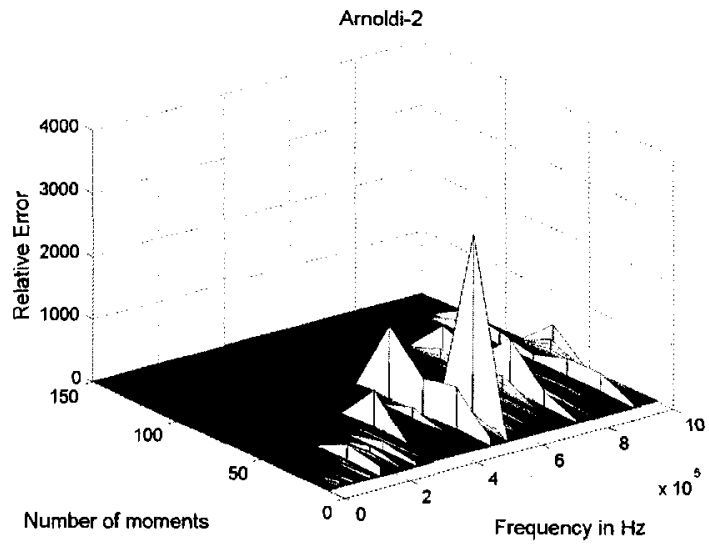

(b) Arnoldi-2

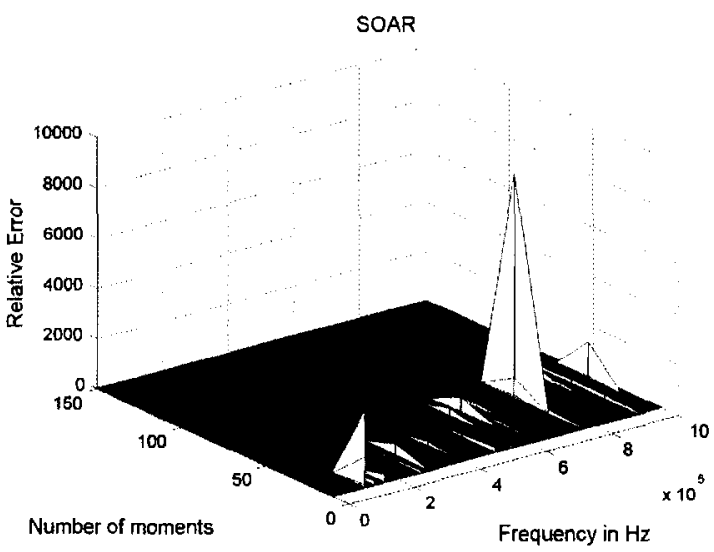

(d) SOAR

Figure C.30: Relative Error for the Frequency Response for 2700-node cantilever, Damping= $10^{-5} \times M$ 
Discussion: The frequency response for all the cantilevers with a damping lower than $10^{-3} \times$ $M$, all exhibited the same trend in that the reduced models provided a good match at lower frequencies, but would provide a better response if a larger number of moments were taken. All three companion forms of the Arnoldi algorithm gave a comparable response and level of error. 


\section{Appendix D}

\section{Additional Time Domain Response for the FEM Cantilever Model}

These are additional results for the time domain analysis not included in the main body of the thesis. All the results for the medium cantilever with 1350 nodes are listed in this chapter, and time domain analysis for all three cantilever models at other magnitudes of damping are also included

\section{D.1 FEM Cantilever Model with Damping $=0 \times M$}

Medium Cantilever built with 1350 tetrahedrons

The time domain response for the full system, the Arnoldi reduced system, and the SOAR reduced 1350-node cantilever is shown in Figures D.1 and D.2 


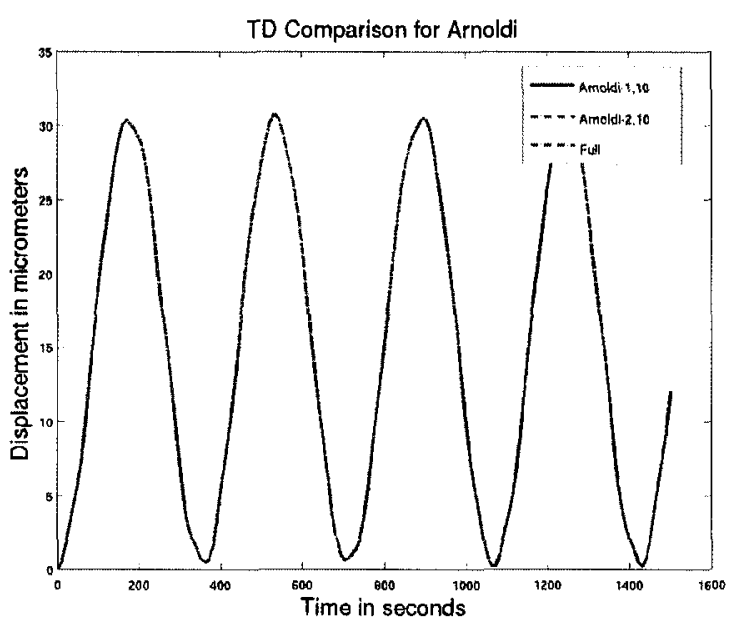

Figure D.1: Comparison of time domain response for the full system and Arnoldi reduced model of a 1350-node Cantilever with Damping $=0 \times M$

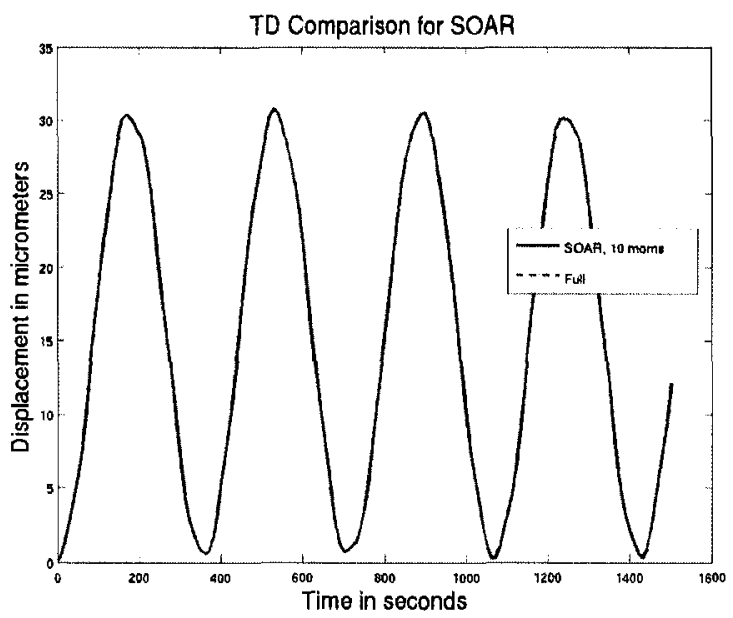

Figure D.2: Comparison of time domain response for the full system and SOAR reduced model of a 1350-node Cantilever with Damping $=0 \times M$ 
Discussion: Figures D.1 and D.2 illustrates that, at 10 moments, there is no discernible difference in the reduced and full time domain responses for both Arnoldi and SOAR.

The relative errors of performing model reduction using the Arnoldi and the SOAR algorithm can be observed in Figure D.3, and it was clearly shown that the SOAR Algorithm did have a lower error at fewer moments. However, the overall magnitude of the relative error must be taken into account as well and even at 10 moments, the error was so small that the benefit of using the SOAR model reduction over Arnoldi was non-existent at no damping. 

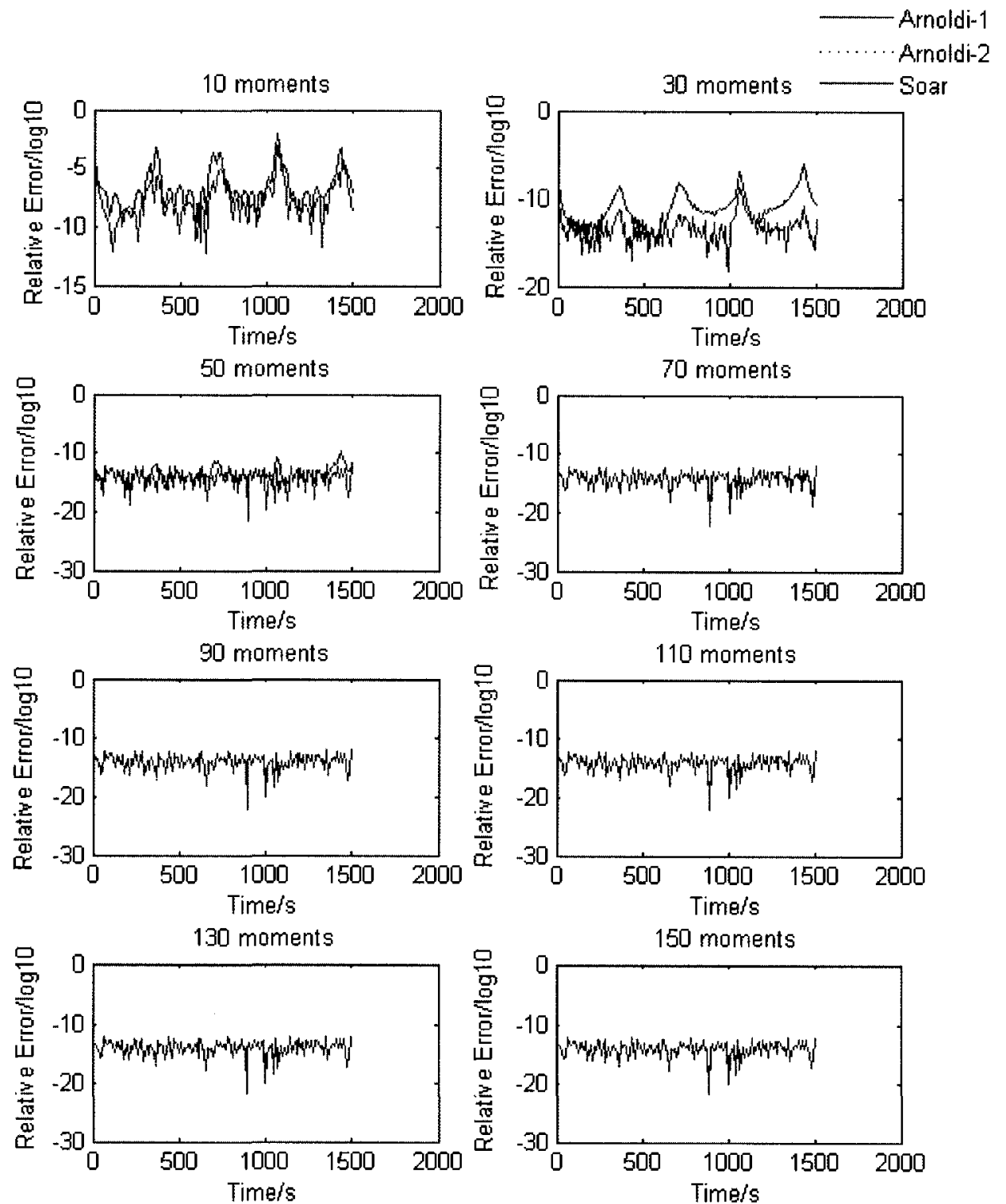

Figure D.3: Relative Error for 1350-node cantilever using Arnoldi at Damping $=0 \times M$ 


\section{D.2 FEM Cantilever Model with Damping $=10^{-2} \times M$}

\section{Medium Cantilever built with 1350-nodes}

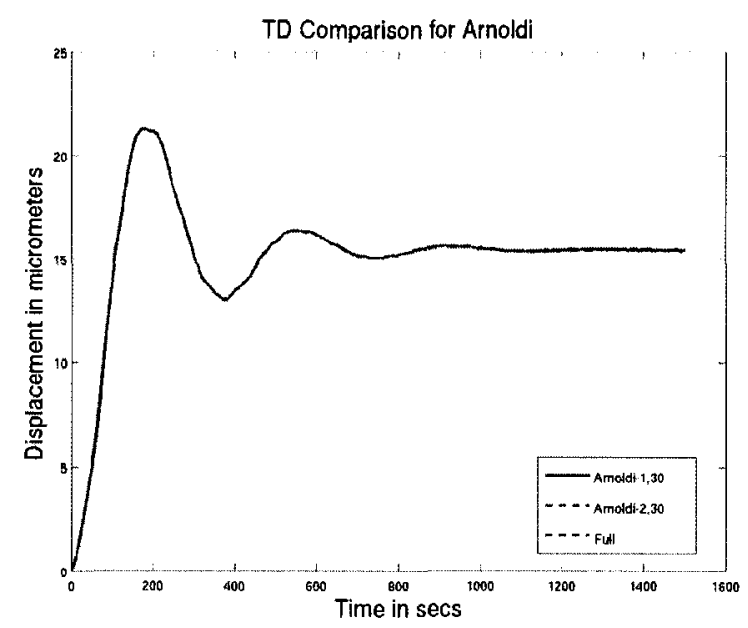

Figure D.4: Comparison of time domain response for the full system and Arnoldi reduced model of a 1350-node Cantilever with Damping $=10^{-2} \times M$, with 30 moments

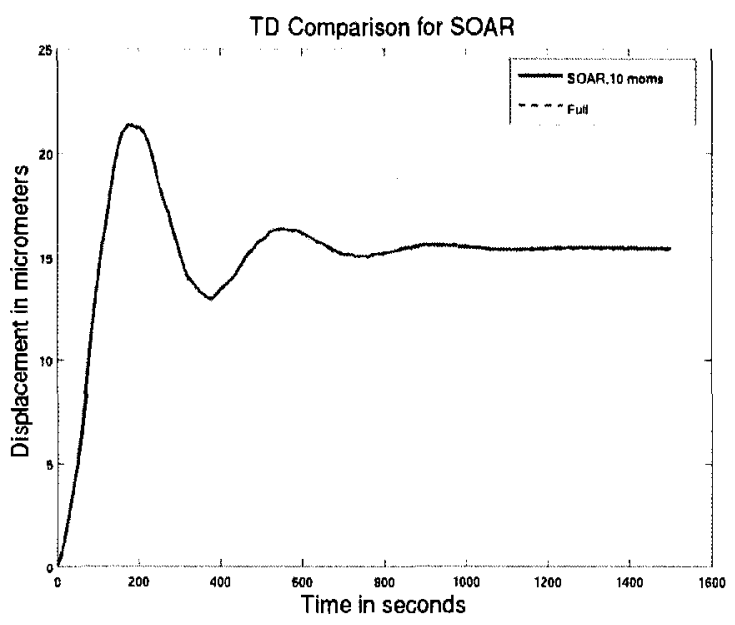

Figure D.5: Comparison of time domain response for the full system and SOAR reduced model of a 1350-node Cantilever with Damping $=10^{-2} \times M$, with 10 moments 
Discussion: For the medium cantilever of 1350-nodes, for a stable response, at least 30 moments was required for both companion forms using the Arnoldi algorithm as shown in Figure D.4 while, even at 10 moments, the SOAR algorithm continued to give a good match to the full model, Figure D.5.

The relative errors of performing model reduction using the Arnoldi and the SOAR algorithm could be observed in Figure D.6, and it was clearly shown that both forms for the Arnoldi algorithm went unstable at 10 moments, while SOAR remains stable and with very low relative error at 10 moments. 

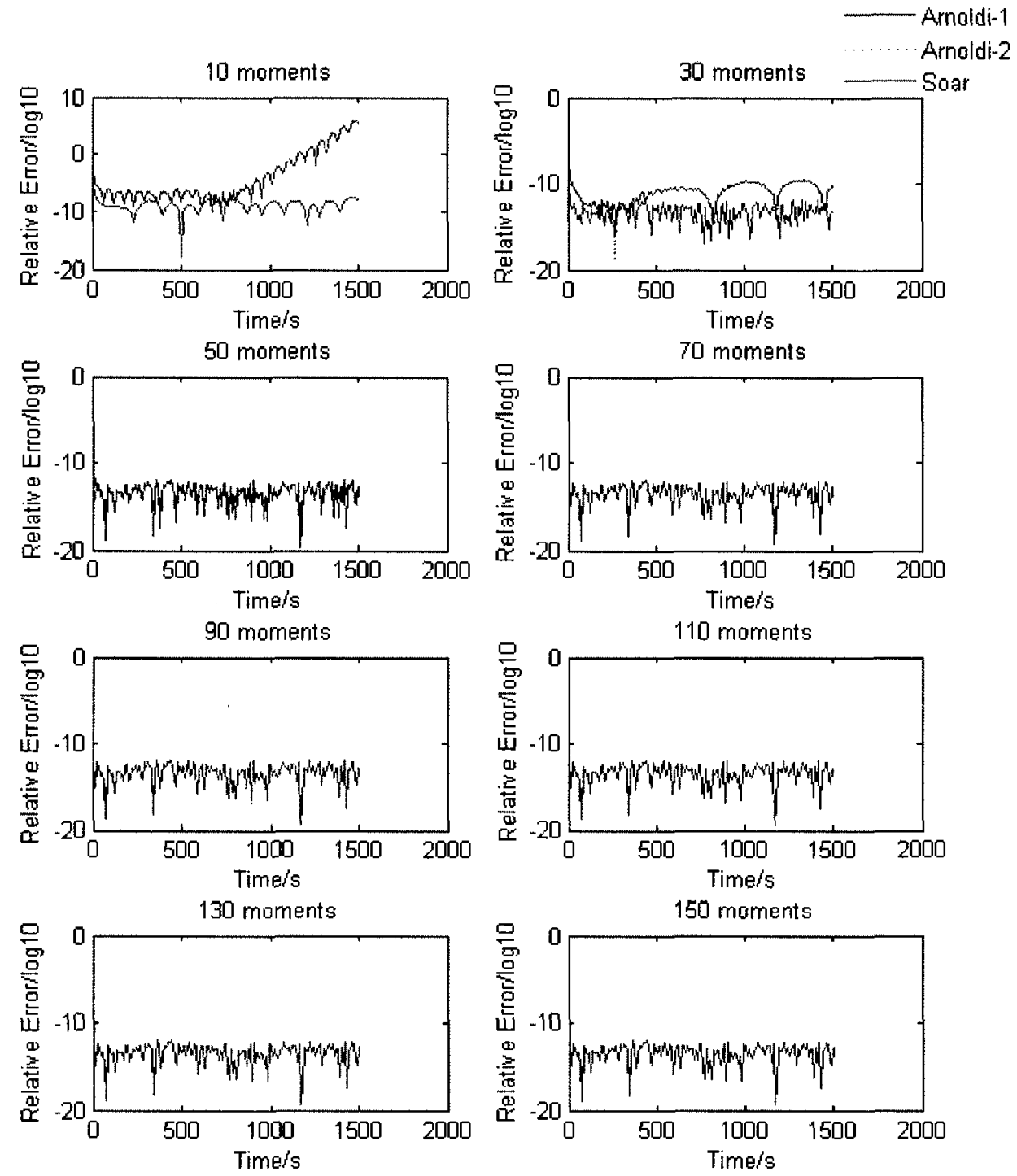

Figure D.6: Relative Error for 1350-node cantilever using Arnoldi at Damping $=10^{-2} \times M$ 


\section{D.3 FEM Cantilever Model with Damping $=10^{-1} \times M$}

\section{Medium Cantilever Built with 1350 tetrahedrons}

The time domain responses for the medium full system, the Arnoldi reduced systems, and the SOAR reduced system, are shown in Figures D.7 and D.8.

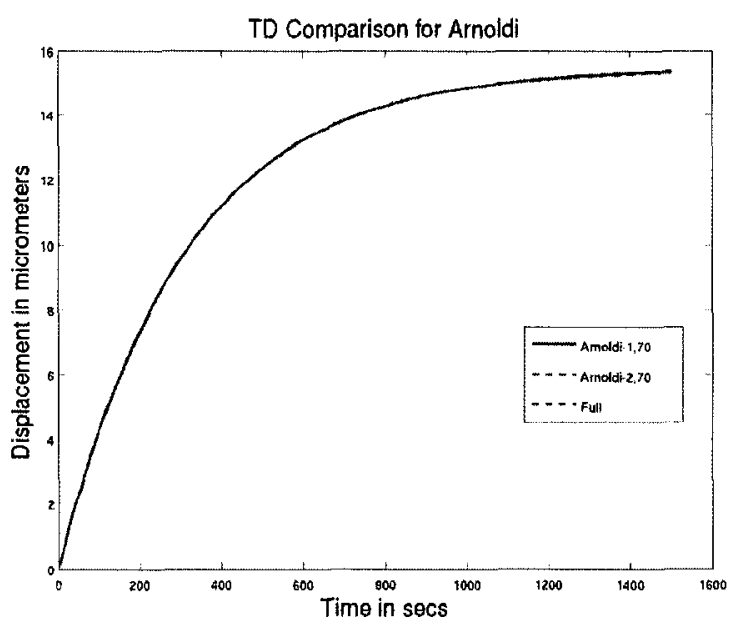

Figure D.7: Comparison of time domain response for the full system and Arnoldi reduced models of a 1350-node Cantilever with Damping $=0.1 \times M$, with 70 moments

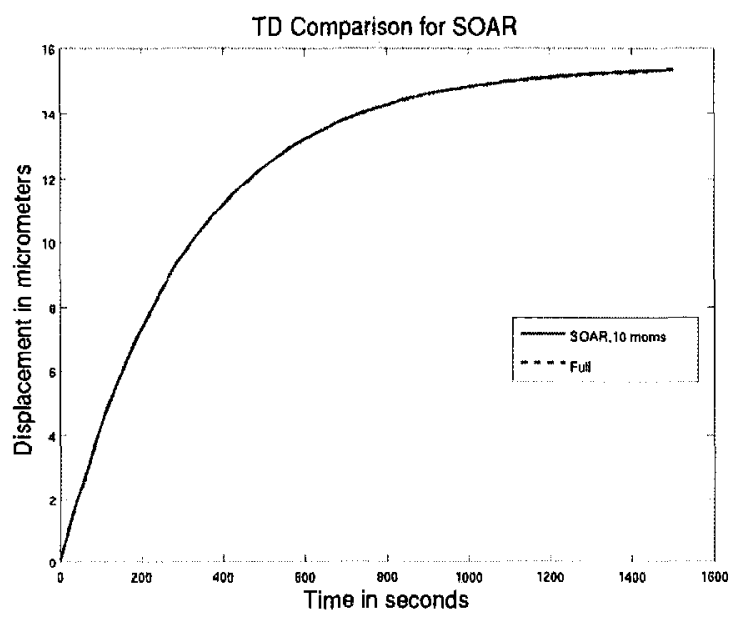

Figure D.8: Comparison of time domain response for the full system and SOAR reduced model of a 1350-node Cantilever with Damping $=0.1 \times M$, with 10 moments 
Discussion: The relative error of the model reductions as shown in Figure D.9 illustrated that the model reduction performed with the Arnoldi algorithm required at least 30 moments with the second companion form configuration, and 70 moments with the first, while SOAR continued to demonstrate sufficiently small error for the 1350 -node cantilever model, even at 10 moments. This was also seen in the Figure D. 7 where all the responses below 70 moments gave highly unstable results. 

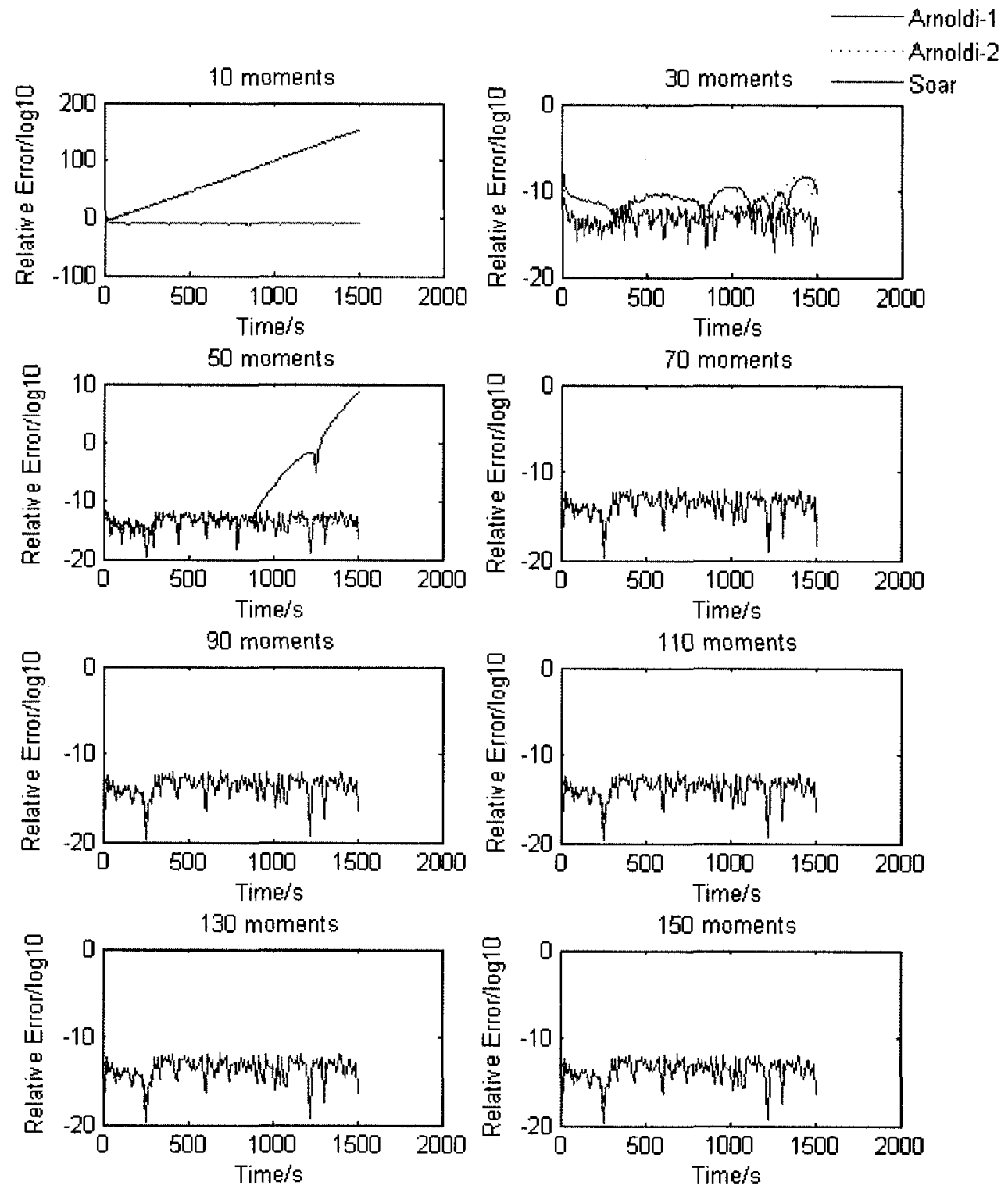

Figure D.9: Relative Error for 1350-node cantilever using Arnoldi at Damping $=0.1 \times M$ 


\section{D.4 FEM Cantilever Model with Damping $=1 \times M$}

When the damping is set to $D=1.0 \times M$, the system matrices are

$$
\underbrace{\left[\begin{array}{cc}
M & M \\
0 & I
\end{array}\right]}_{A}\left[\begin{array}{c}
\ddot{u}(t) \\
\dot{u}(t)
\end{array}\right]+\underbrace{\left[\begin{array}{cc}
0 & K \\
-I & 0
\end{array}\right]}_{B}\left[\begin{array}{c}
\dot{u}(t) \\
u(t)
\end{array}\right]=\underbrace{\left[\begin{array}{c}
F(t) \\
0
\end{array}\right]}_{E}, y(t)=\left[\begin{array}{ll}
0 & L
\end{array}\right]\left[\begin{array}{c}
\dot{u}(t) \\
u(t)
\end{array}\right]
$$

and

$$
\underbrace{\left[\begin{array}{cc}
M & 0 \\
0 & I
\end{array}\right]}_{A}\left[\begin{array}{c}
\ddot{u}(t) \\
\dot{u}(t)
\end{array}\right]+\underbrace{\left[\begin{array}{cc}
M & K \\
-I & 0
\end{array}\right]}_{B}\left[\begin{array}{c}
\dot{u}(t) \\
u(t)
\end{array}\right]=\underbrace{\left[\begin{array}{c}
F(t) \\
0
\end{array}\right]}_{E}, y(t)=\left[\begin{array}{ll}
0 & L
\end{array}\right]\left[\begin{array}{c}
\dot{u}(t) \\
u(t)
\end{array}\right]
$$

\section{D.4.1 Small Cantilever Built with 540 tetrahedrons}

The 540-node cantilever will now be considered with the damping set to $D=1.0 \times M$, and model reductions using Arnoldi and SOAR are performed again in the time domain.

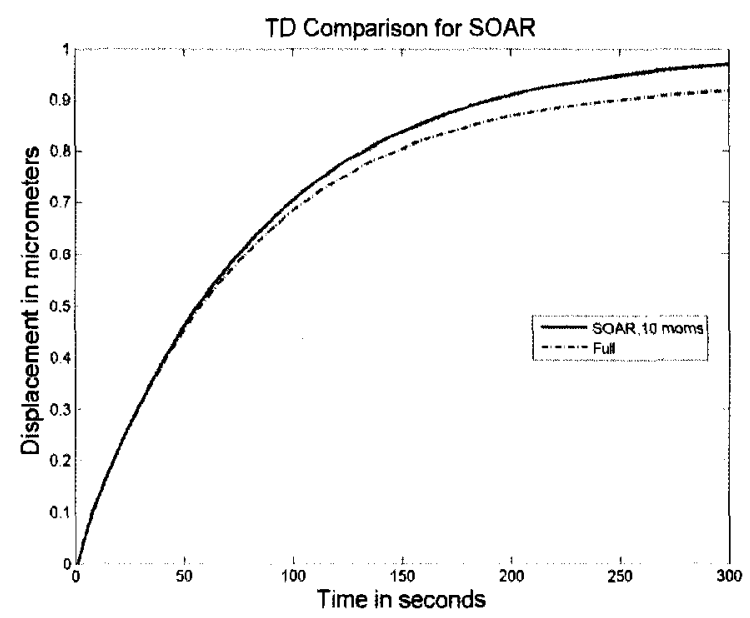

Figure D.10: Comparison of time domain response for the full system and SOAR reduced model of a 540-node Cantilever with Damping=1 $\times M$, with 10 moments

Discussion: The small cantilever, at this level of damping, was unable to achieve any stable time domain response using both companion forms of Arnoldi, while, at 10 moments, the 
SOAR algorithm gave a good match to the full model, Figure D.10. On evaluation of the relative errors in the model reductions using the Arnoldi and the SOAR algorithms, as can be observed in Figure D.11, and it was clearly shown that both forms for the Arnoldi algorithm goes unstable even at 150 moments, while SOAR remained stable and with very low relative error with very little moments. 

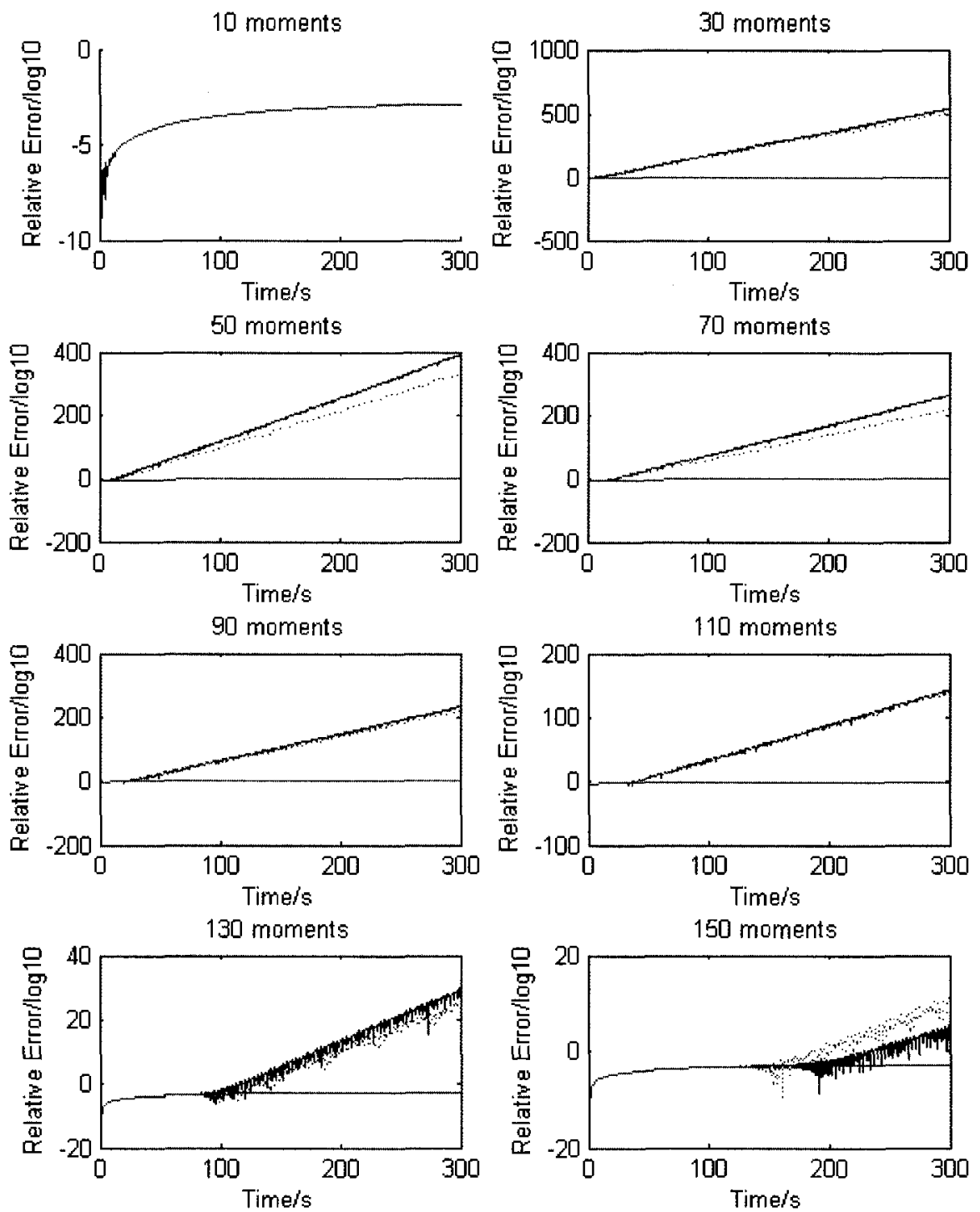

Figure D.11: Relative Error for 540-node cantilever using Arnoldi at Damping $=1 \times M$ 


\section{D.4.2 Medium Cantilever Built with 1350 tetrahedrons}

The damping is now set to $D=1.0 \times M$, and model reductions using Arnoldi and SOAR are performed again in the time domain.

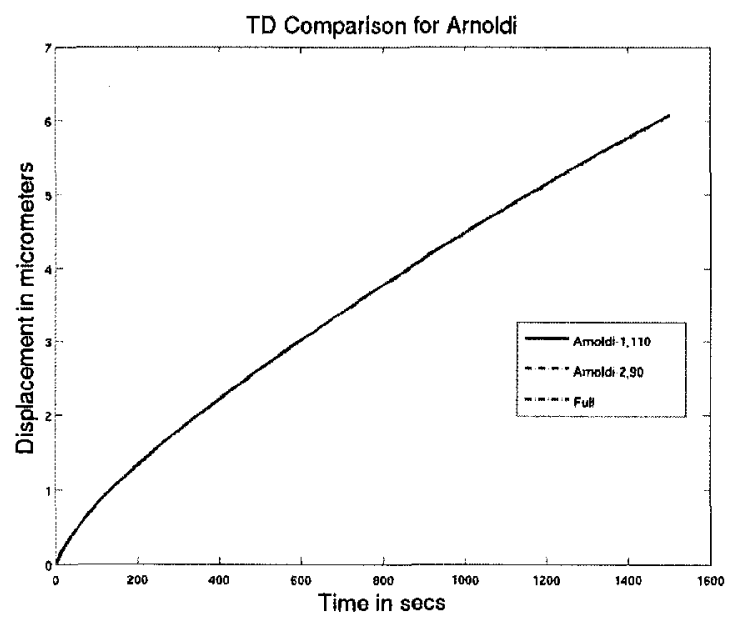

Figure D.12: Comparison of time domain response for the full system and Arnoldi reduced model of a 1350-node Cantilever with Damping $=1 \times M$, with 90 and 110 moments

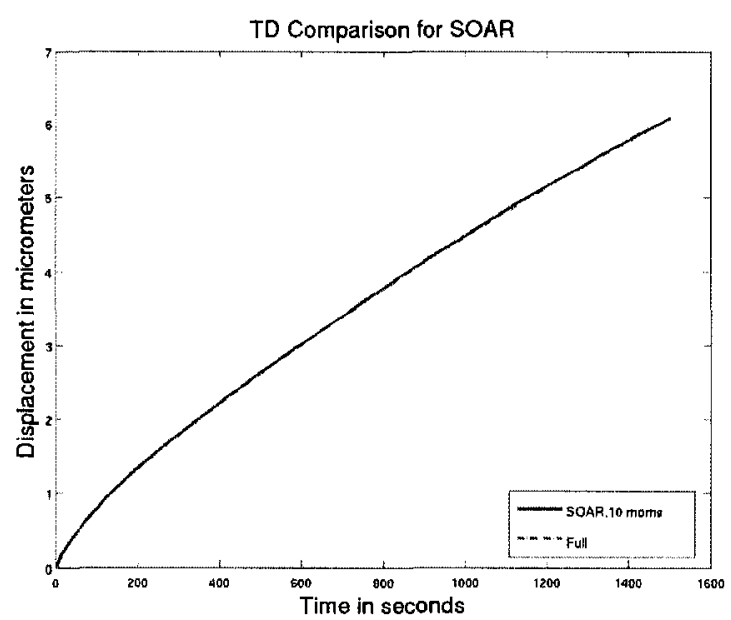

Figure D.13: Comparison of time domain response for the full system and SOAR reduced model of a 1350-node Cantilever with Damping $=1 * \mathrm{M}$, with 10 moments 
Discussion: For the medium beam of 1350-nodes, for a stable response, it appeared that at least 50 moments was required for both companion forms using the Arnoldi algorithm as shown in Figure D.12 while, even at 10 moments, the SOAR algorithm gave a good match to the full model, Figure D.13. However, on closer inspection of the relative errors in the model reductions using the Arnoldi and the SOAR algorithms as can be observed in Figure D.14, and it was clearly shown that both forms for the Arnoldi algorithm went unstable up to 90 moments, while SOAR remained stable and with very low relative error at 10 moments. 

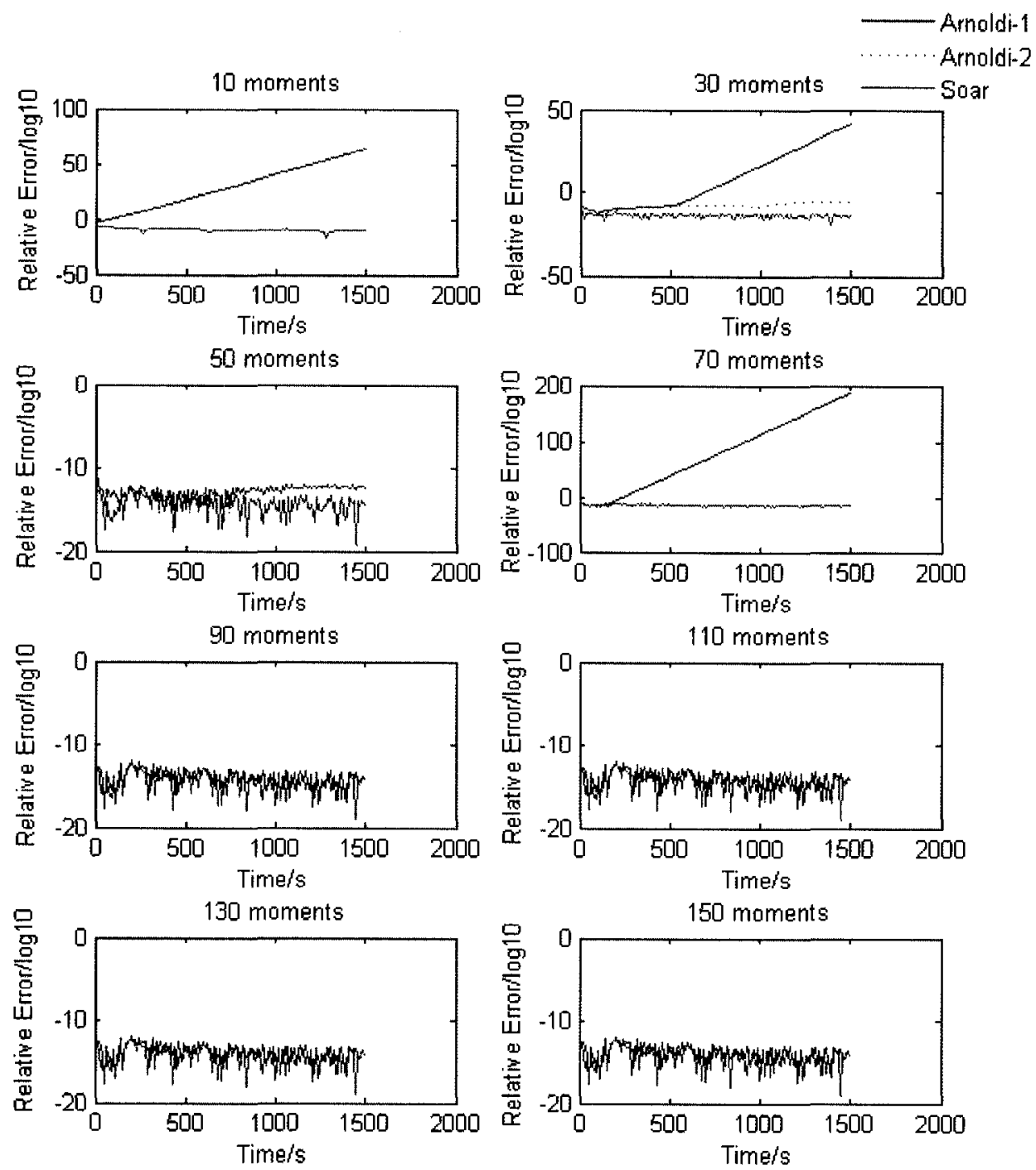

Figure D. 14: Relative Error for 1350-node cantilever using Arnoldi at Damping $=1 \times M$ 


\section{D.4.3 Large Cantilever Built with 2700 tetrahedrons}

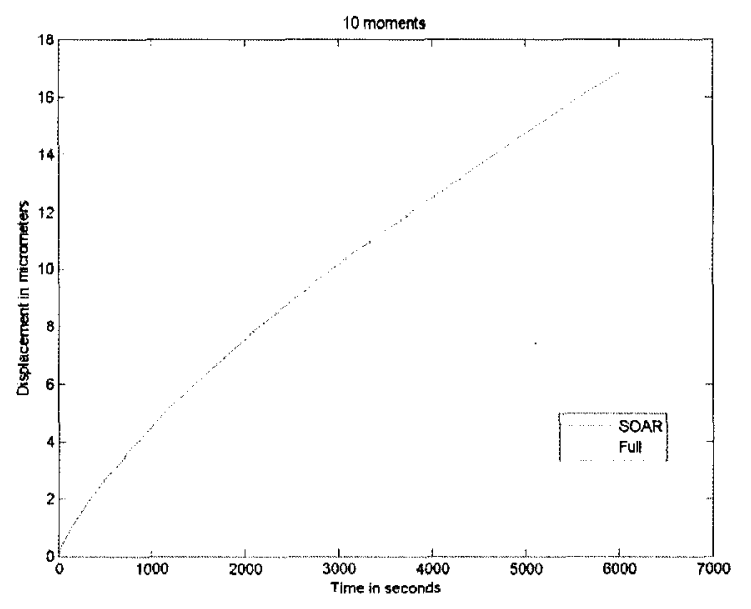

Figure D.15: Comparison of time domain response for the full system and SOAR reduced model of a 2700-node Cantilever with Damping $=1 \times M$, with 10 moments

Discussion: For the large beam of 2700-nodes, no stable response could be achieved up to 450 moments using the Arnoldi algorithm, at which point the benefits of using model reduction techniques became obsolete and the computational time to achieve the time domain response approached that of the full system. SOAR, on the other hand, gave an exceptional match to the full response at a low 10 moments, Figure D.15.

The relative errors in the model reductions using the Arnoldi and the SOAR algorithms as can be observed in Figure D.16, and it was clearly shown that both forms for the Arnoldi algorithm remained unstable at 150 moments, while SOAR remained stable and with very low relative error at 10 moments. 

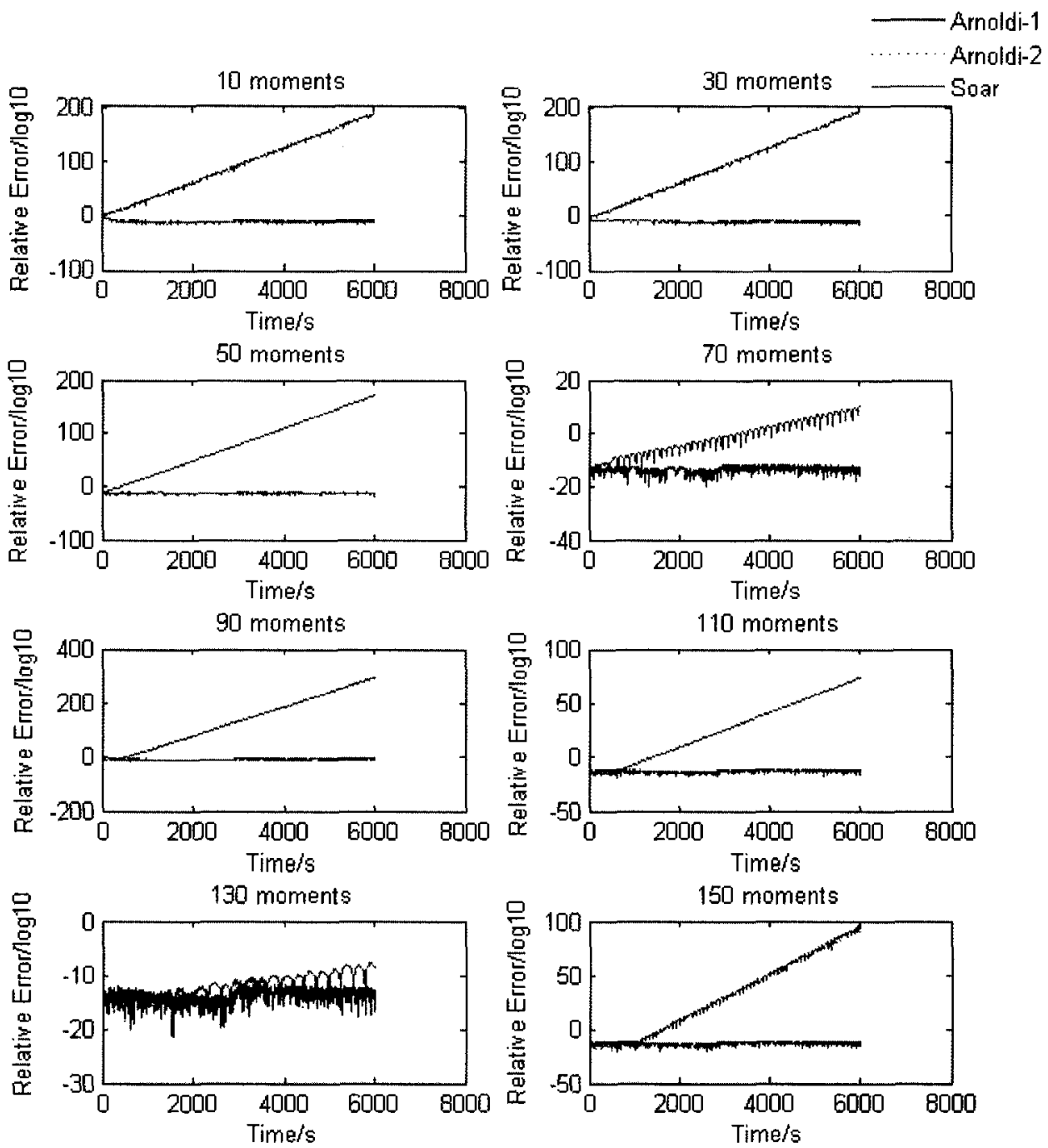

Figure D.16: Relative Error for 2700-node cantilever using Arnoldi at Damping $=1 \times M$ 


\section{D.5 Cantilever Model with Damping $\leq D=10^{-3} \times M$}

The Damping of the system is now set such that $D=10^{-3} \times M$, and the same comparisons were performed. The system matrices for the two stable Arnoldi configurations are now

$$
\underbrace{\left[\begin{array}{cc}
M & 10^{-3} * M \\
0 & I
\end{array}\right]}_{A}\left[\begin{array}{c}
\ddot{u}(t) \\
\dot{u}(t)
\end{array}\right]+\underbrace{\left[\begin{array}{cc}
0 & K \\
-I & 0
\end{array}\right]}_{B}\left[\begin{array}{c}
\dot{u}(t) \\
u(t)
\end{array}\right]=\underbrace{\left[\begin{array}{c}
F(t) \\
0
\end{array}\right]}_{E}, y(t)=\left[\begin{array}{ll}
0 & L
\end{array}\right]\left[\begin{array}{l}
\dot{u}(t) \\
u(t)
\end{array}\right]
$$

and

$$
\underbrace{\left[\begin{array}{cc}
M & 0 \\
0 & I
\end{array}\right]}_{A}\left[\begin{array}{c}
\ddot{u}(t) \\
\dot{u}(t)
\end{array}\right]+\underbrace{\left[\begin{array}{cc}
10^{-3} * M & K \\
-I & 0
\end{array}\right]}_{B}\left[\begin{array}{c}
\dot{u}(t) \\
u(t)
\end{array}\right]=\underbrace{\left[\begin{array}{c}
F(t) \\
0
\end{array}\right]}_{E}, y(t)=\left[\begin{array}{ll}
0 & L
\end{array}\right]\left[\begin{array}{c}
\dot{u}(t) \\
u(t)
\end{array}\right]
$$

\section{D.5.1 Small Cantilever Built with 540-nodes}

At this point, the comparison for the time domain response between the Arnoldi algorithms and SOAR and the full model, dampings ranging from $10^{-5} \geq$ damping $\leq 10^{-3}$ will be considered and the results are shown in Figures D.17 and D.18. 


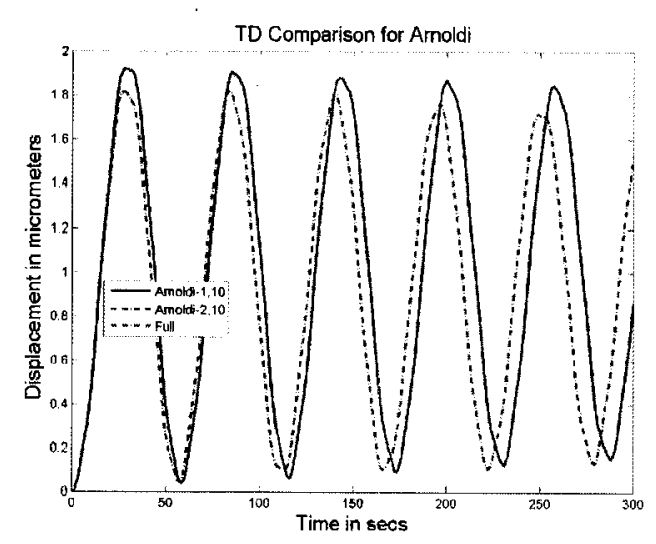

(a) Arnoldi at Damping $==10^{-3}$

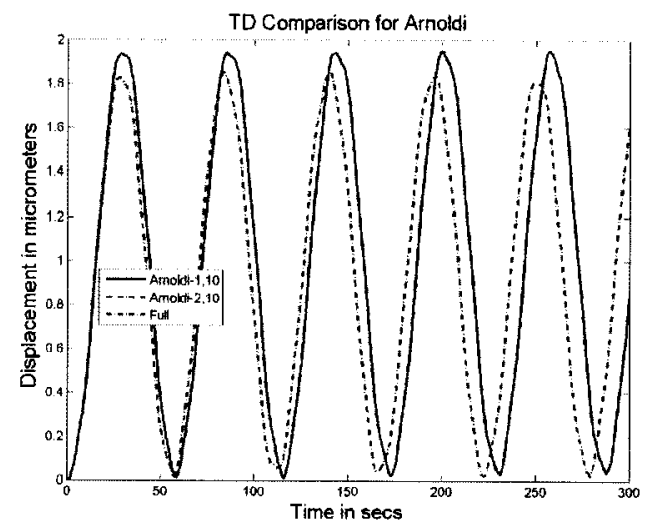

(b) Arnoldi at Damping $=10^{-4}$

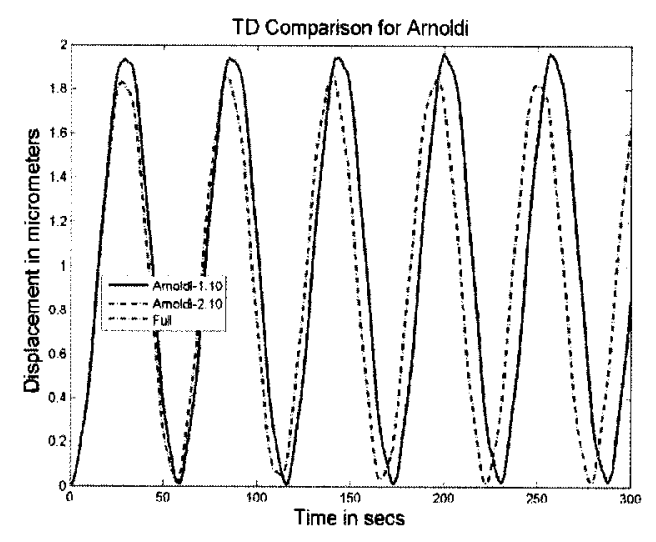

(c) Arnoldi at Damping $=10^{-5}$

Figure D.17: Comparison of time domain Response for the full system and Arnoldi reduced models of a 540-node Cantilever with Damping $=10^{-3} \times M, 10^{-4} \times M$ and $10^{-5} \times M$, with 10 moments 


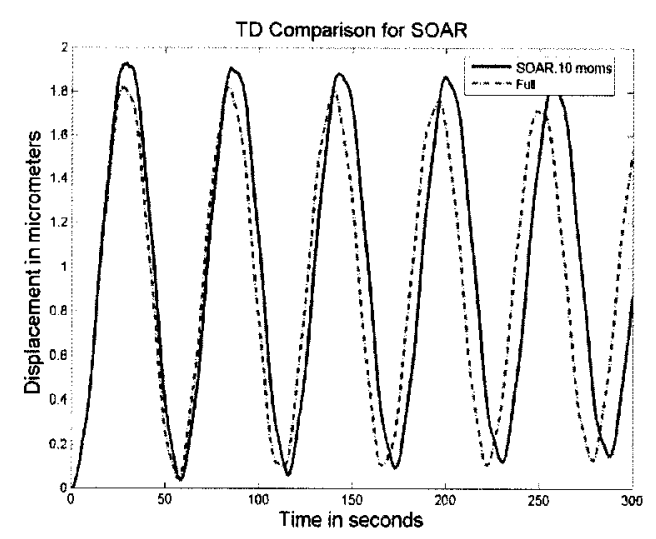

(a) SOAR at Damping $==10^{-3}$

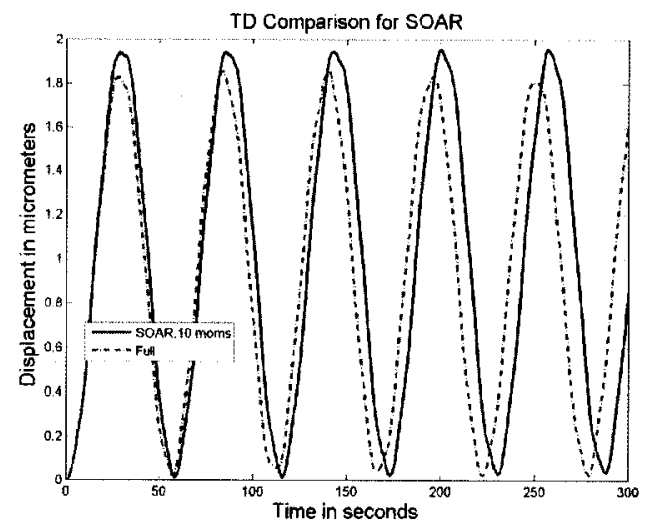

(b) SOAR at Damping $=10^{-4}$

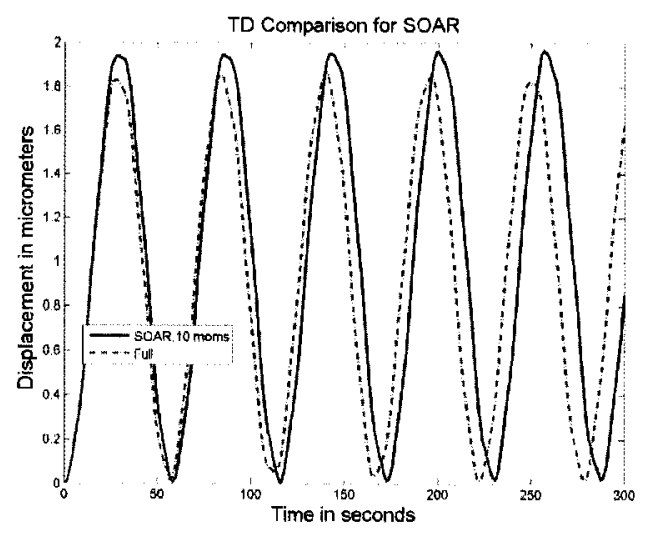

(c) SOAR at Damping $=10^{-5}$

Figure D.18: Comparison of time domain response for the full system and SOAR reduced model of a 540-node Cantilever with Damping $=10^{-3} \times M, 10^{-4} \times M$ and $10^{-5} \times M$, with 10 moments 
Discussion: At damping where $D \leq 0.01 \times M$, all model reduction techniques implemented match the full model with a very small number of moments, and as can be seen in Figures D.19, D.20, and D.21, the evaluation of the relative errors for all three model reductions performed here had minimal and comparable errors. 

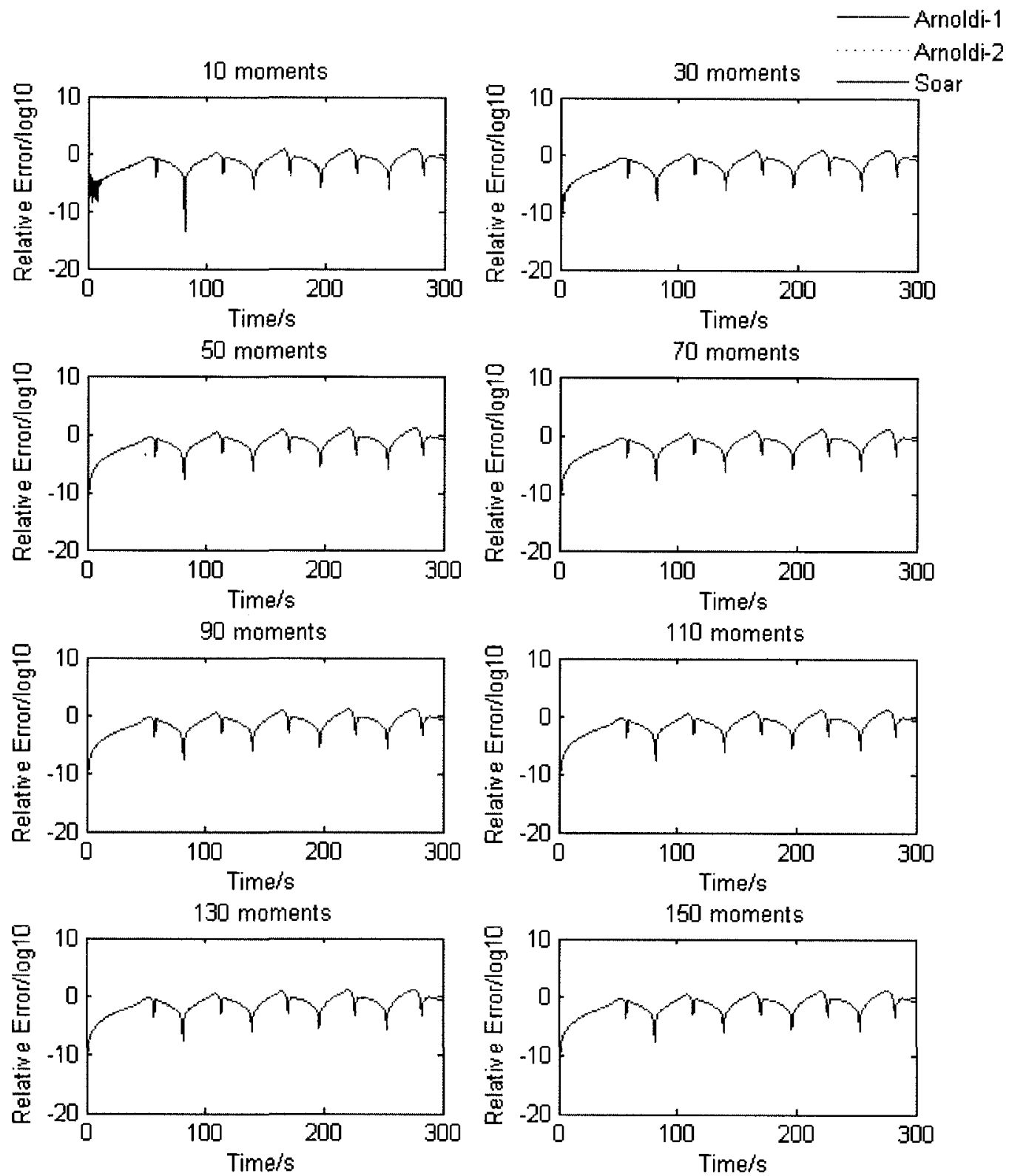

Figure D.19: Relative Error for 540-node cantilever using Arnoldi at Damping $=10^{-3} \times M$ 

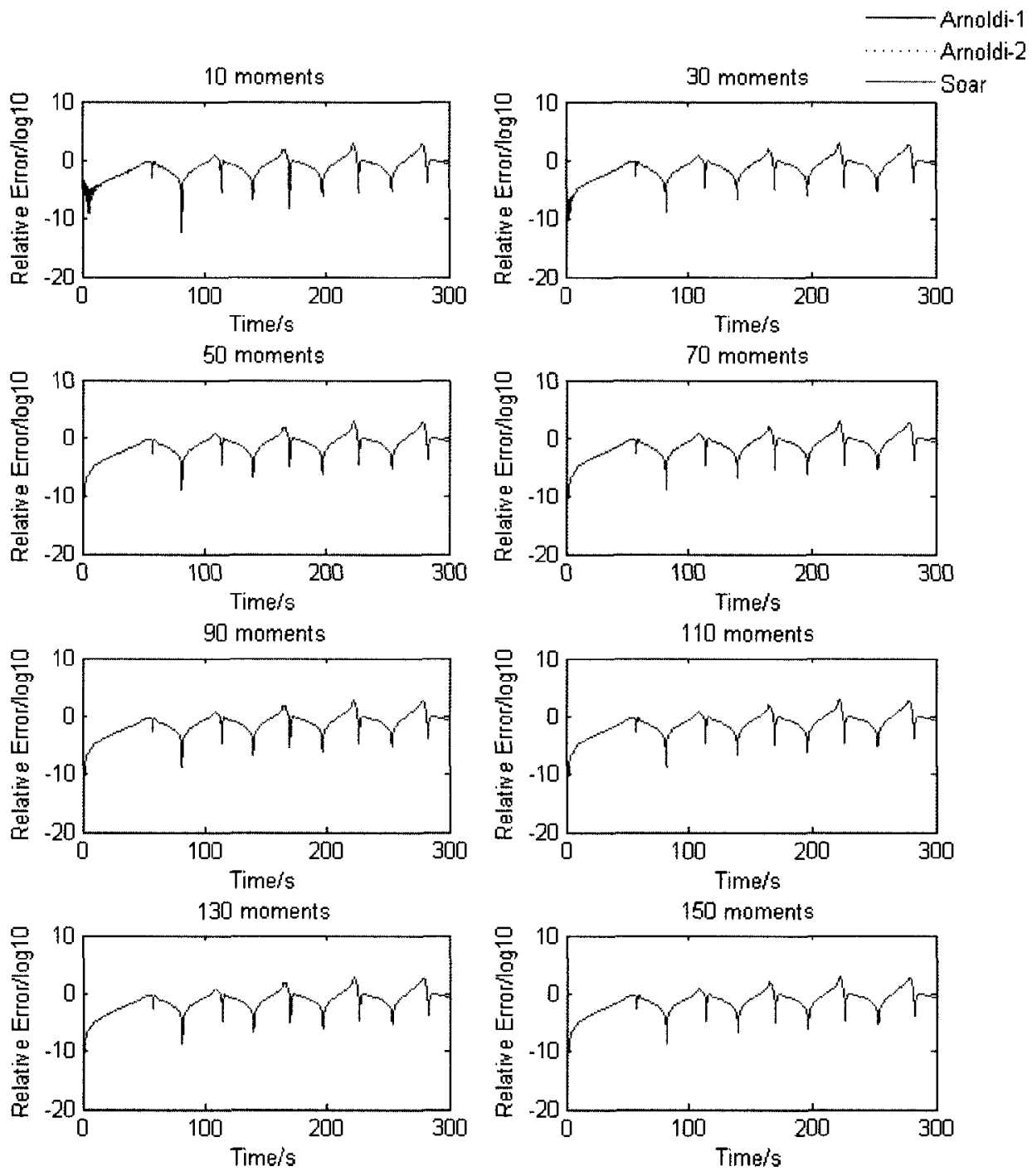

Figure D.20: Relative Error for 540-node cantilever using Arnoldi at Damping $=10^{-4} \times M$ 

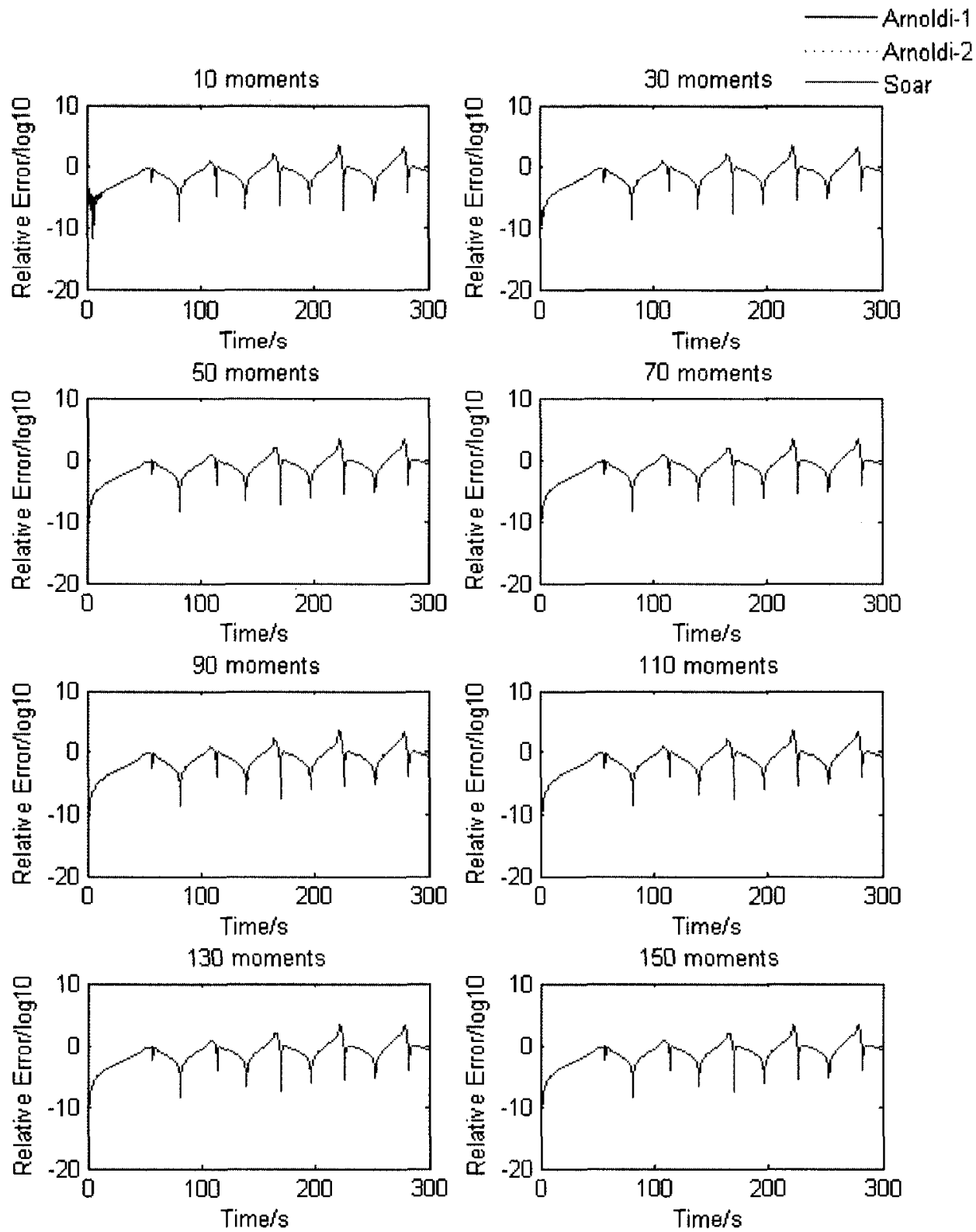

Figure D.21: Relative Error for 540-node cantilever using Arnoldi at Damping $=10^{-5} \times M$ 


\section{D.5.2 Medium Cantilever Built with 1350-nodes}

The time domain responses at dampings ranging from $10^{-5} \geq$ damping $\leq 10^{-3}$ between the Arnoldi algorithms and SOAR and the full model, are shown in Figures D.22 and D.23.

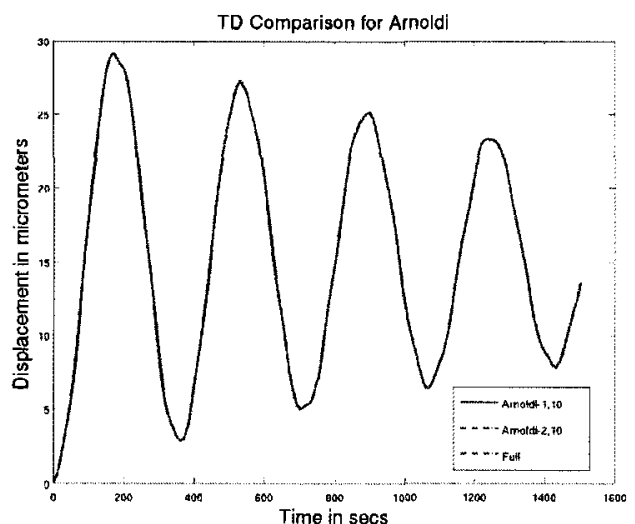

(a) Arnoldi at Damping $==10^{-3}$

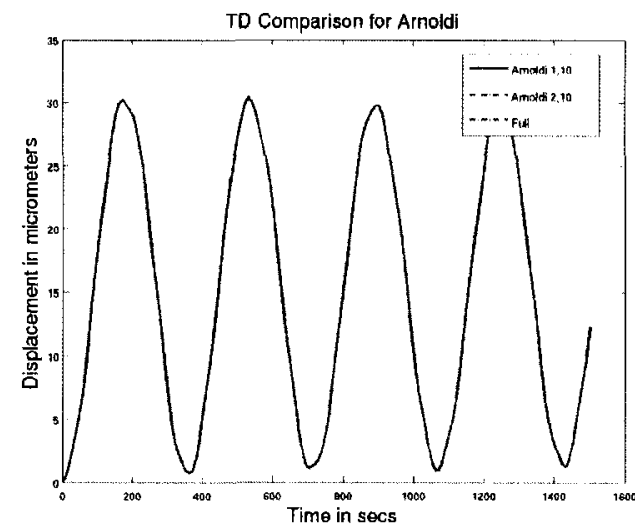

(b) Arnoldi at Damping $=10^{-4}$

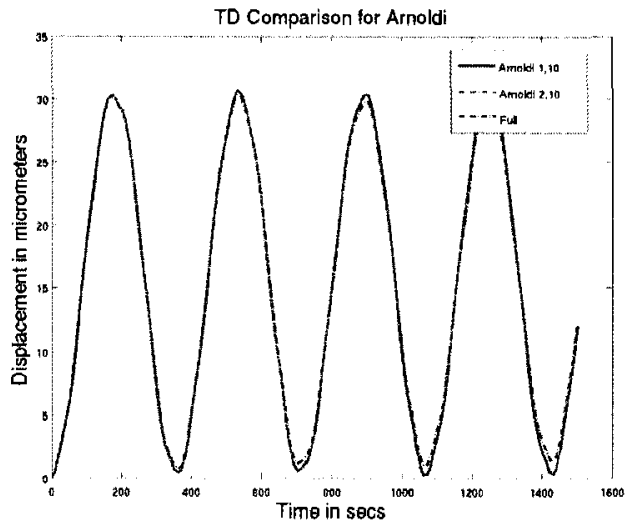

(c) Arnoldi at Damping $=10^{-5}$

Figure D.22: Comparison of time domain Response for the full system and Arnoldi reduced models of a 1350-node Cantilever with Damping $=10^{-3} \times M, 10^{-4} \times M$ and $10^{-5} \times M$, with 10 moments 


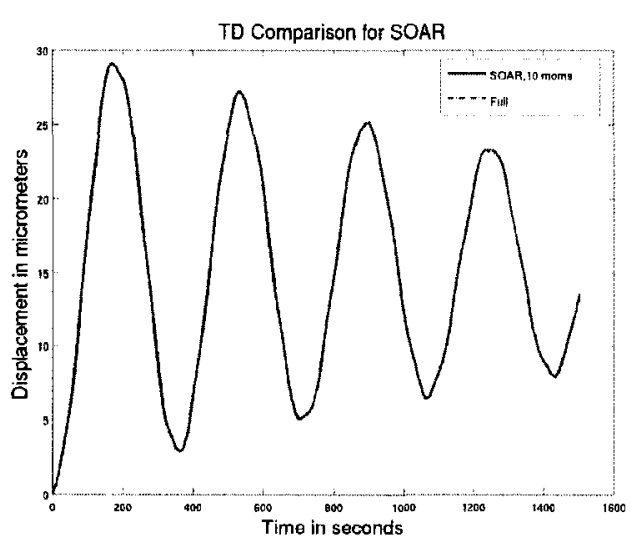

(a) SOAR at Damping $==10^{-3}$

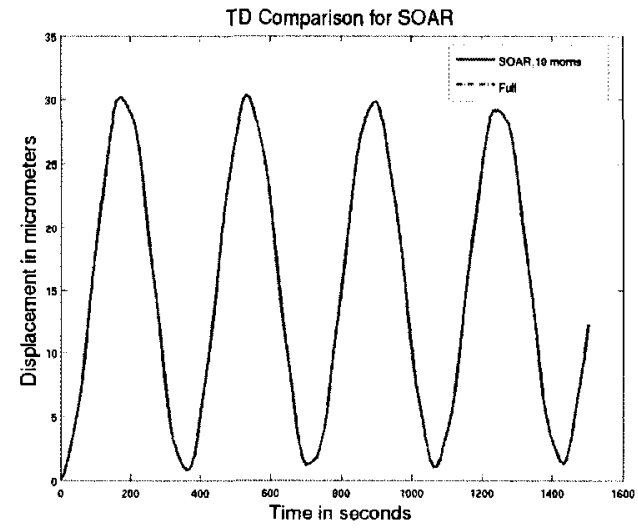

(b) SOAR at Damping $=10^{-4}$

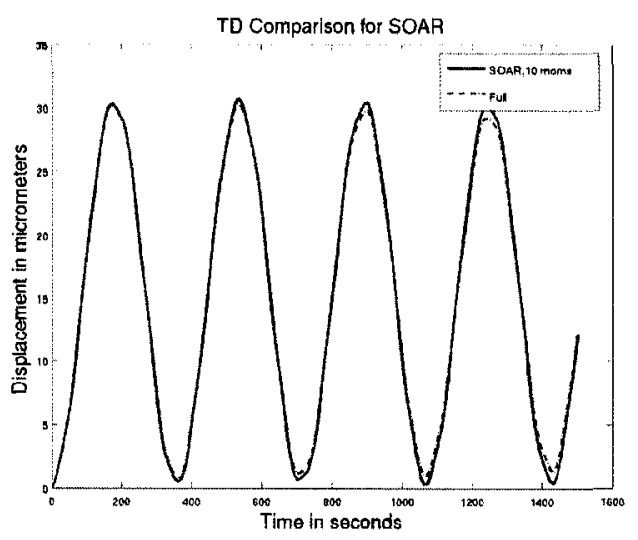

(c) SOAR at Damping $=10^{-5}$

Figure D.23: Comparison of time domain response for the full system and SOAR reduced model of a 1350-node Cantilever with Damping $=10^{-3} \times M, 10^{-4} \times M$ and $10^{-5} \times M$, with 10 moments 
Discussion: At damping where $D \leq 0.01 \times M$, all model reduction techniques implemented match the full model with a very small number of moments, and as can be seen in Figures D.24, D.25, and D.26, the comparison of relative errors for all three model reductions performed here had minimal error compared to the full response. 

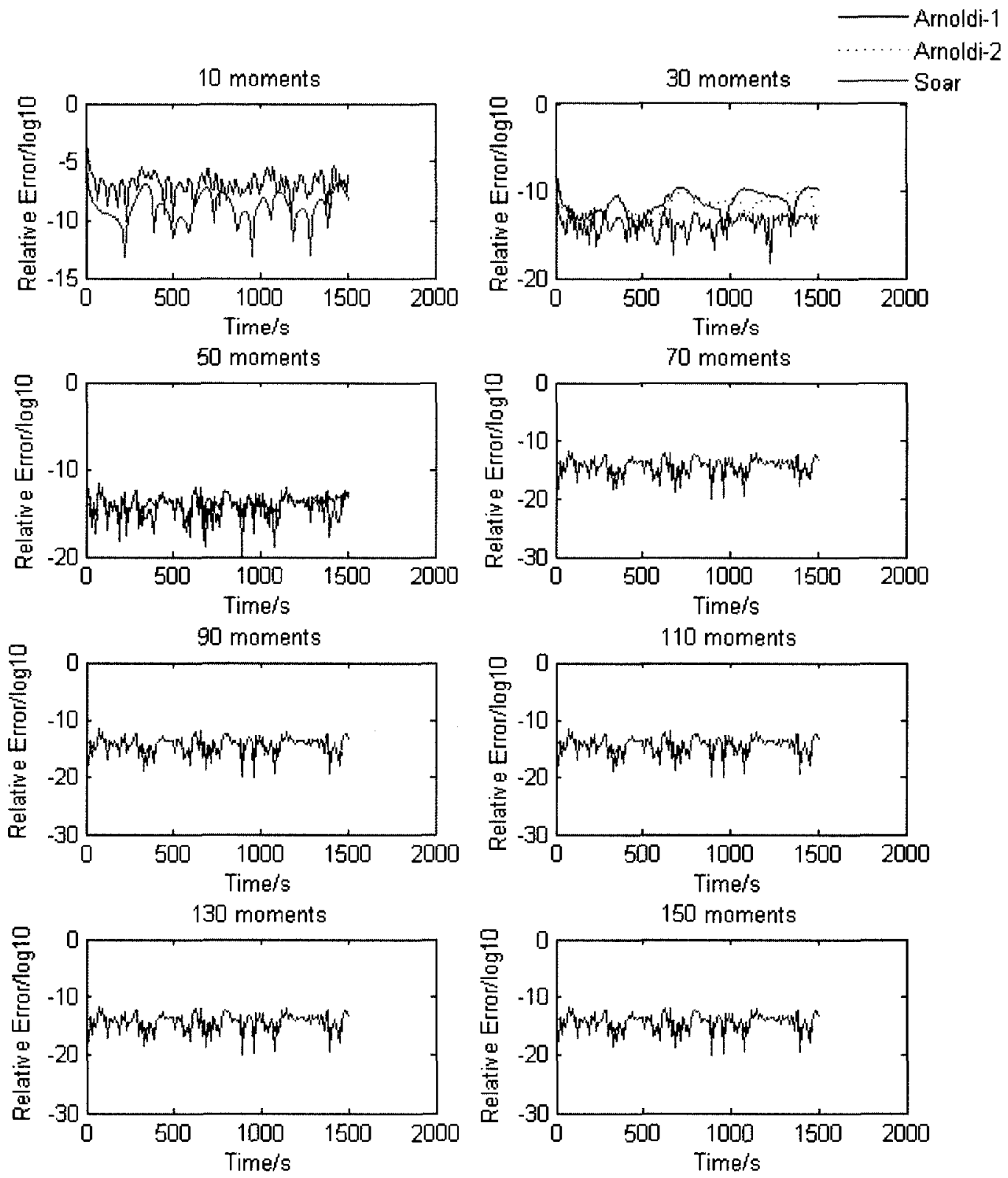

Figure D.24: Relative Error for 1350-node cantilever using Arnoldi at Damping $=10^{-3} \times M$ 

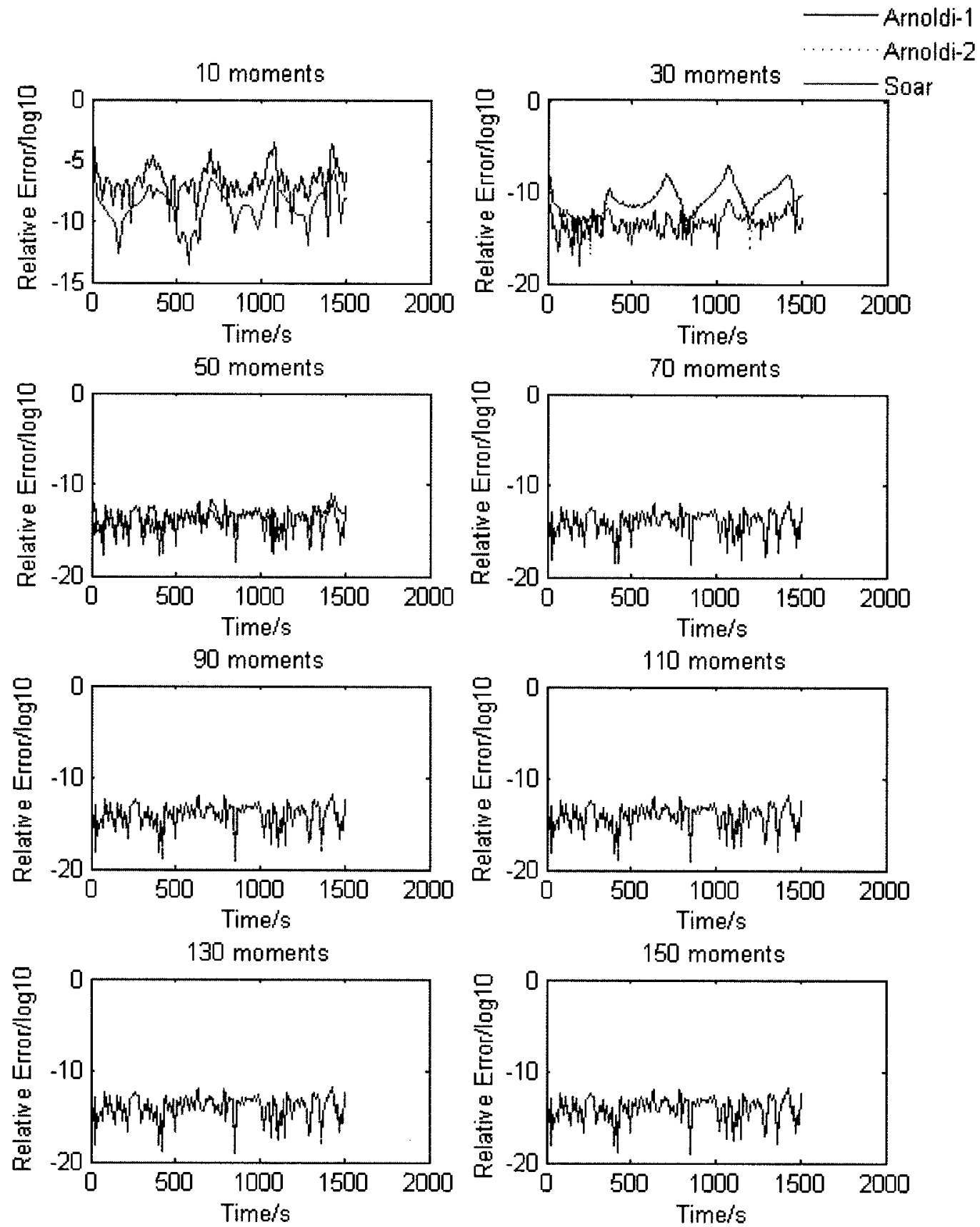

Figure D.25: Relative Error for 1350-node cantilever using Arnoldi at Damping $=10^{-4} \times M$ 

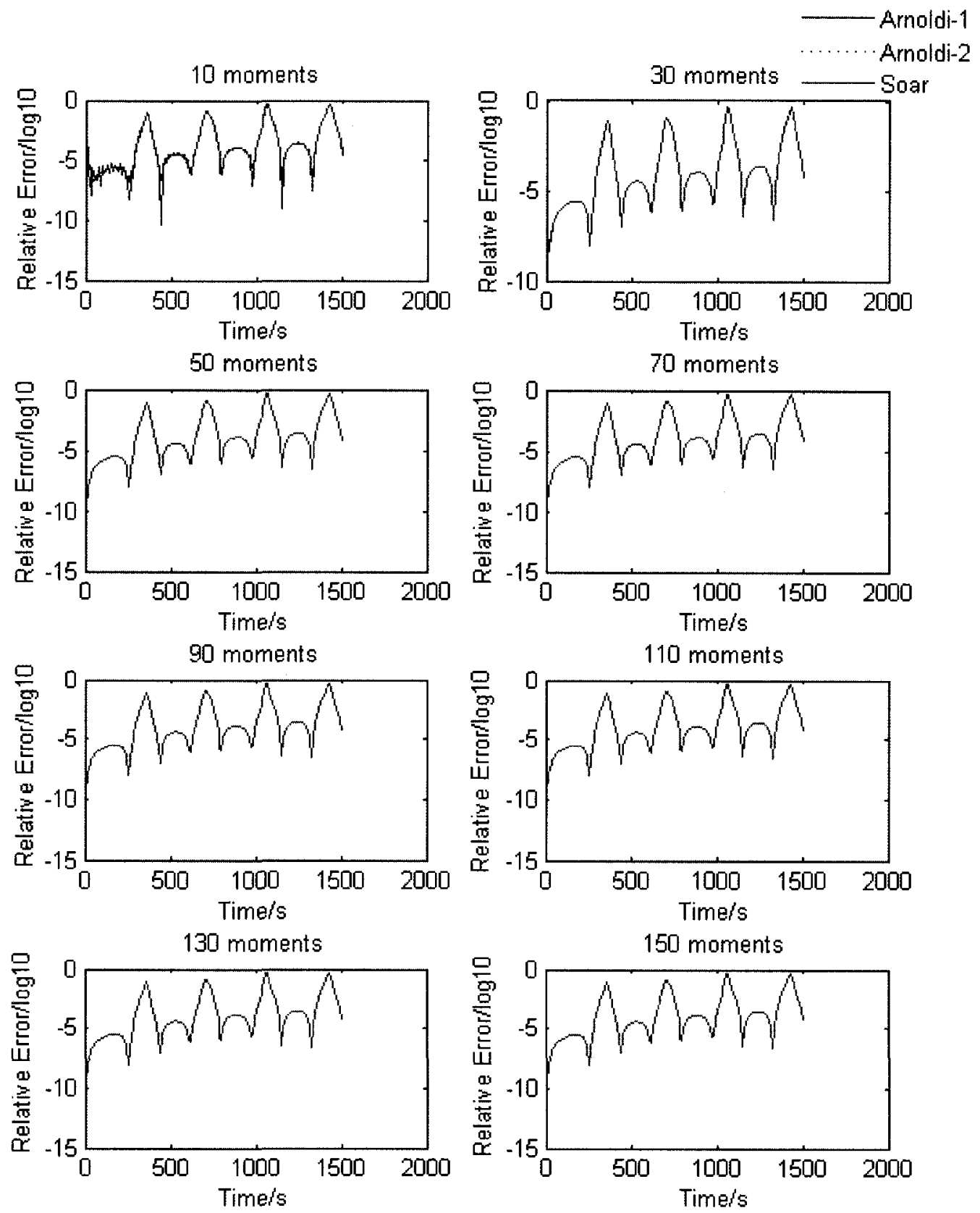

Figure D.26: Relative Error for 1350-node cantilever using Arnoldi at Damping $=10^{-5} \times M$ 


\section{D.5.3 Large Cantilever Built with 2700-nodes}

The time domain response between the Arnoldi algorithms and SOAR and the full model, dampings ranging from $10^{-5} \geq$ damping $\leq 10^{-3}$ will be considered for the large cantilever with 2700-nodes and the results are shown in Figures D.27 and D.28.

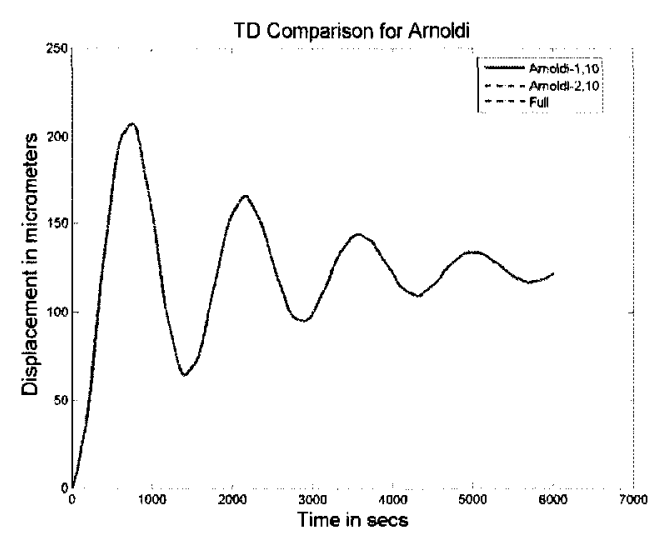

(a) Arnoldi at Damping $==10^{-3}$

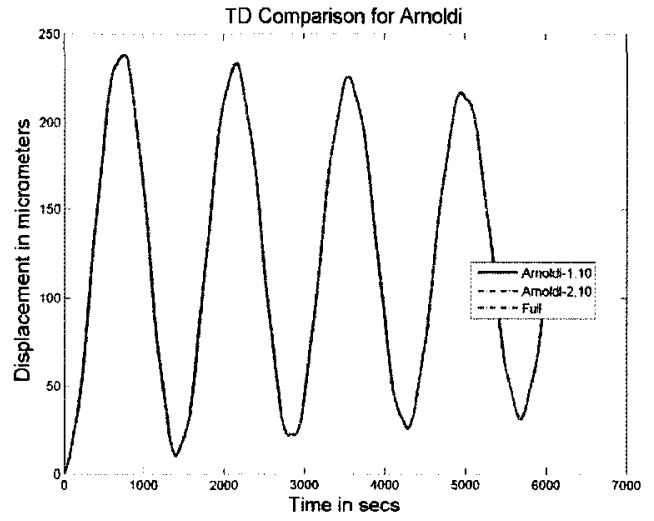

(b) Arnoldi at Damping $=10^{-4}$

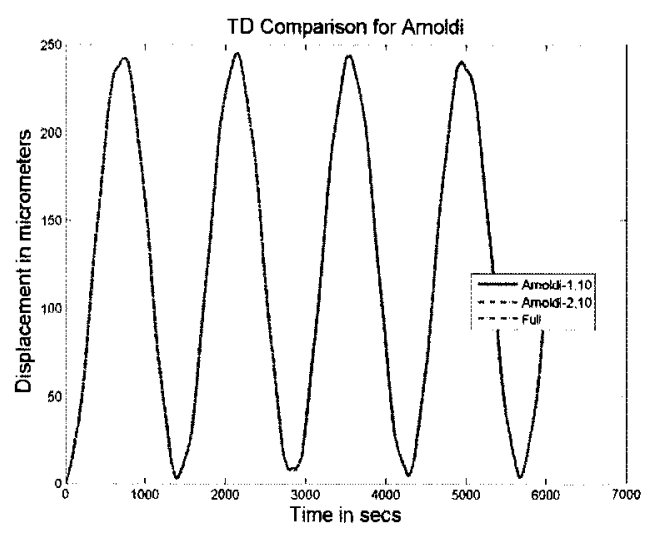

(c) Arnoldi at Damping $=10^{-5}$

Figure D.27: Comparison of time domain Response for the full system and Arnoldi reduced models of a 2700-node Cantilever with Damping $=10^{-3} \times M, 10^{-4} \times M$ and $10^{-5} \times M$, with 10 moments 


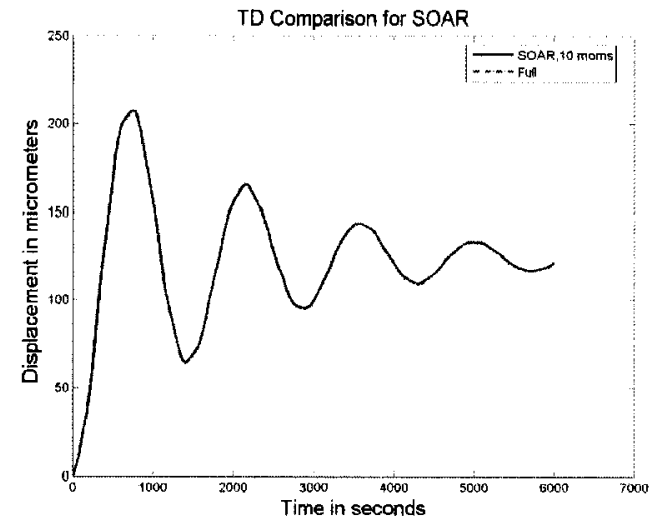

(a) SOAR at Damping $==10^{-3}$

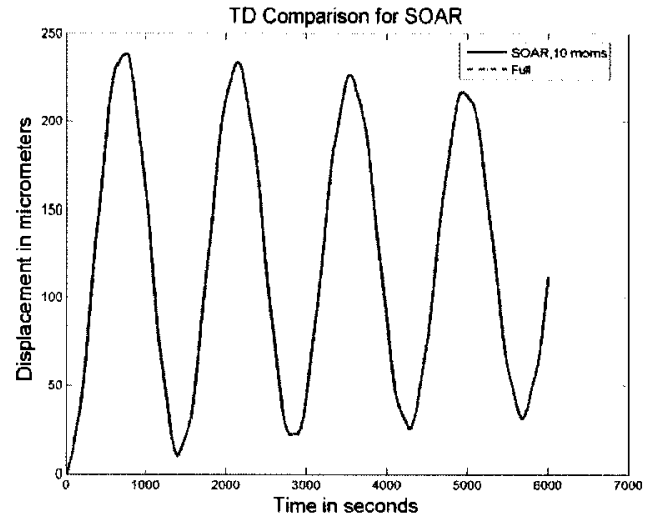

(b) SOAR at Damping $=10^{-4}$

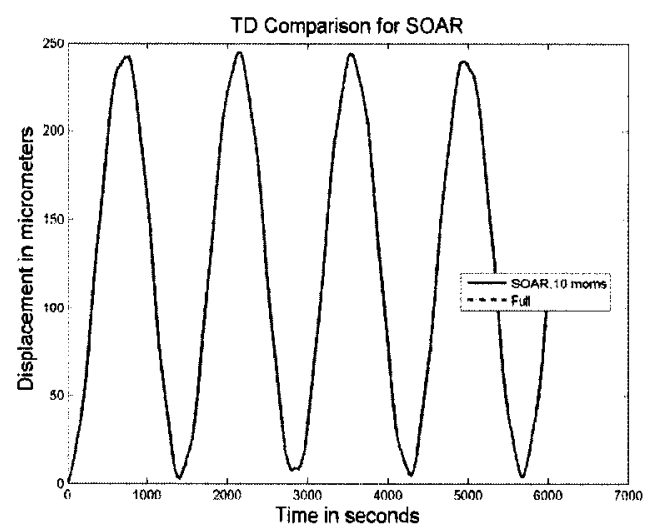

(c) SOAR at Damping $=10^{-5}$

Figure D.28: Comparison of time domain response for the full system and SOAR reduced model of a 2700-node Cantilever with Damping $=10^{-3} \times M, 10^{-4} \times M$ and $10^{-5} \times M$, with 10 moments 
Discussion: Here it was observed that, for Arnoldi, a good match was achievable at 10 moments but the system exhibits unstable properties until 70 moments for a damping $D=$ $10^{-3} \times M$ as shown in Figure D.29.

At dampings where $D \leq 10^{-3} \times M$, all model reduction techniques implemented match the full model with a very small number of moments, and as can be seen in Figures D.30, and D.31, the comparison of relative errors for the last two model reductions performed here had minimal error compared to the full response. 

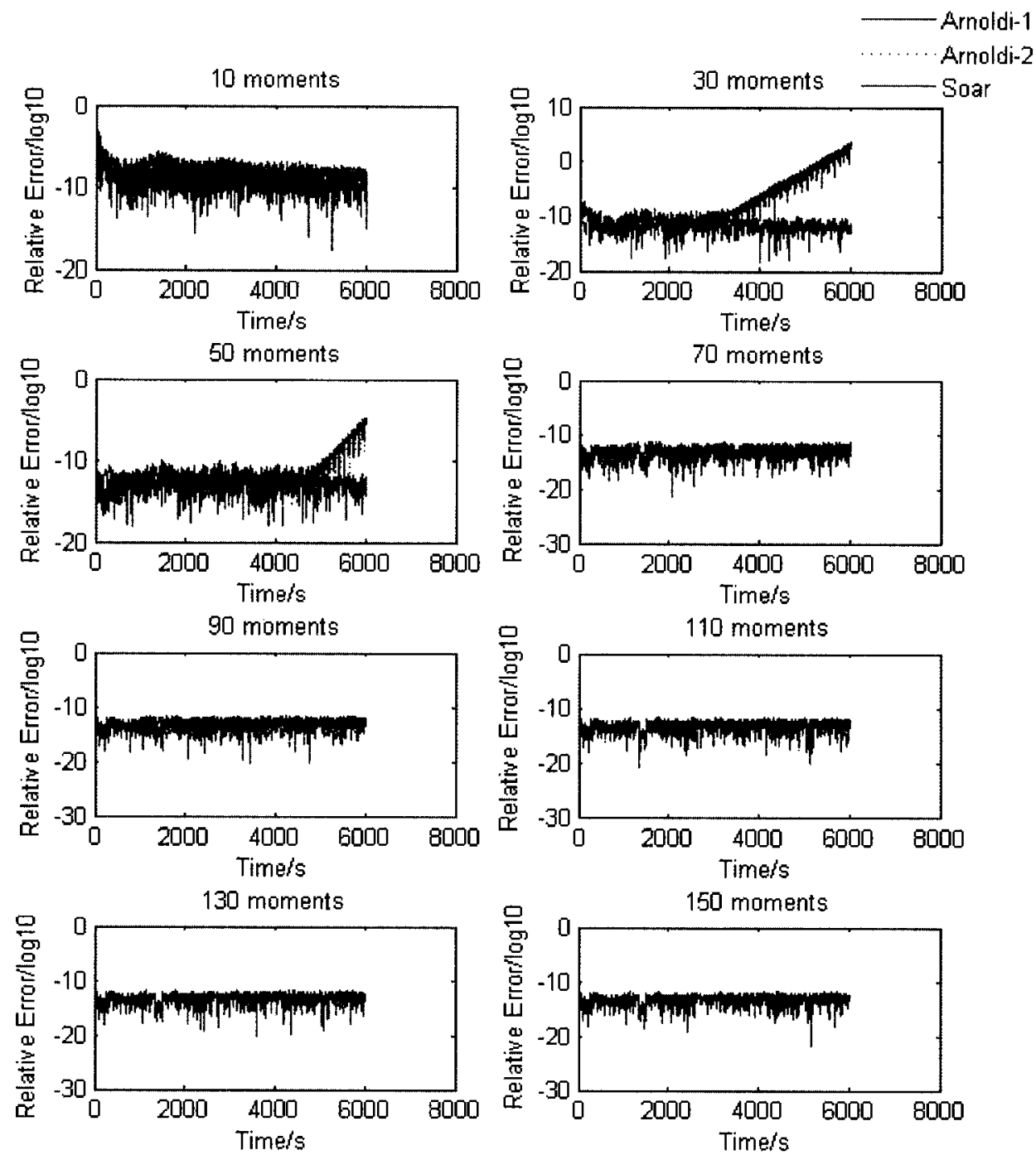

Figure D.29: Relative Error for 2700-node cantilever using Arnoldi at Damping $=10^{-3} \times M$ 

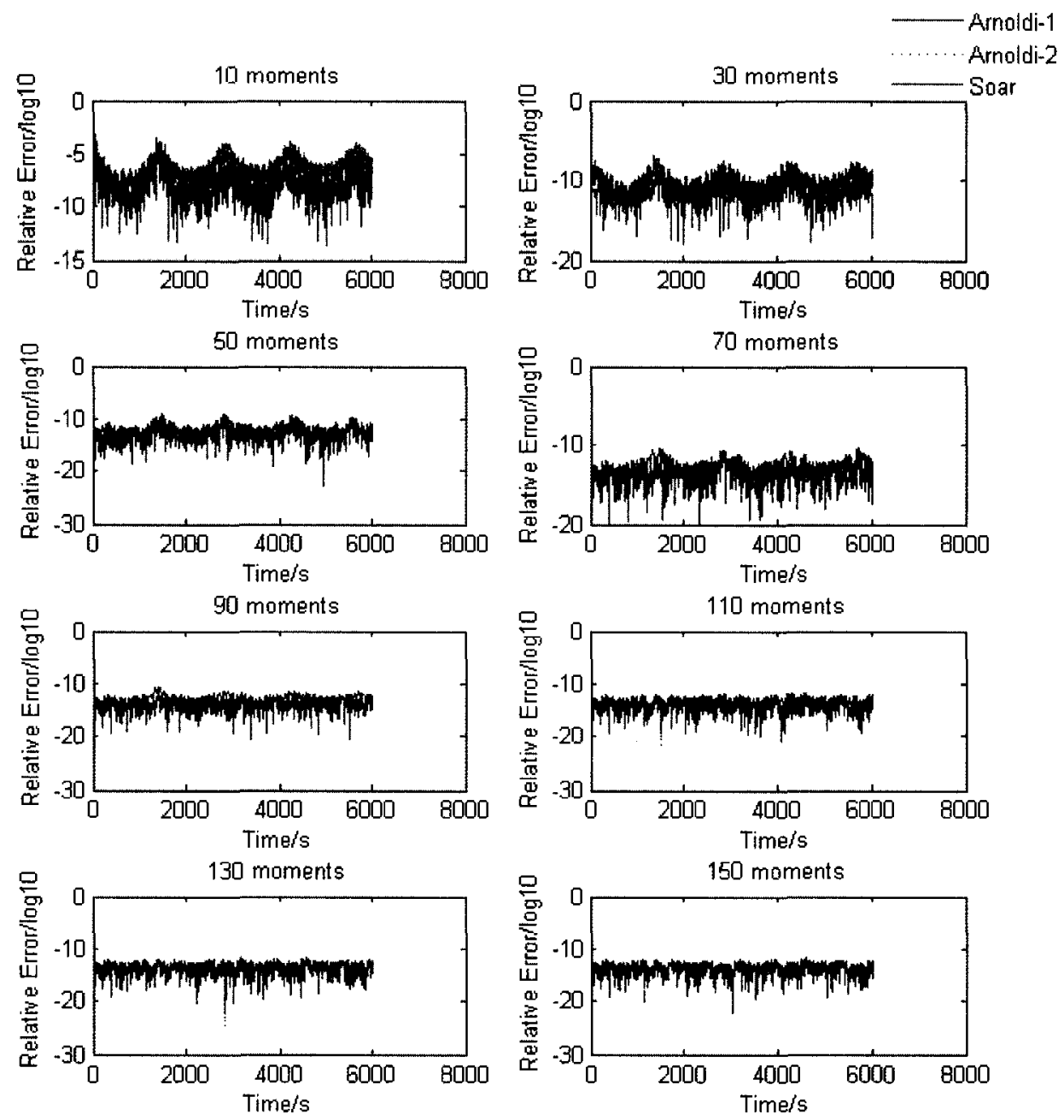

Figure D.30: Relative Error for 2700-node cantilever using Arnoldi at Damping $=10^{-4} \times M$ 

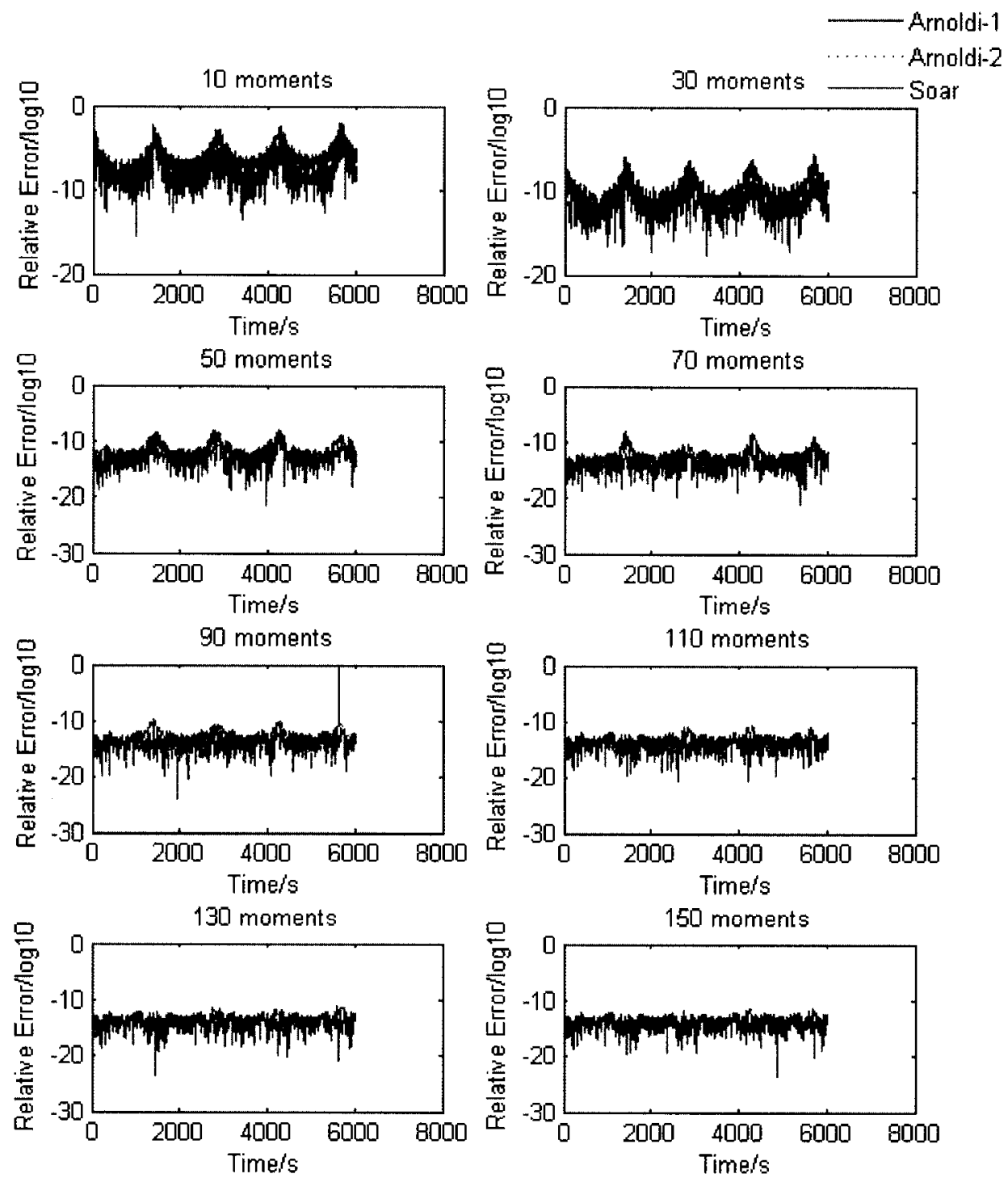

Figure D.31: Relative Error for 2700-node cantilever using Arnoldi at Damping $=10^{-5} \times M$ 


\section{Appendix E}

\section{Further Eigenvalue Numerical Results}

Shown below are further results that prove that, as discussed in Chapter 7, all the eigenvalues of reduced damped systems formed with an undamped orthogonal $Q$-matrix generated in the Arnoldi algorithm or the SOAR procedure lie on the stable left-hand side of the complex plane.

Recall, for stability there should be no poles of significant value in the right-hand side of the complex plane. 

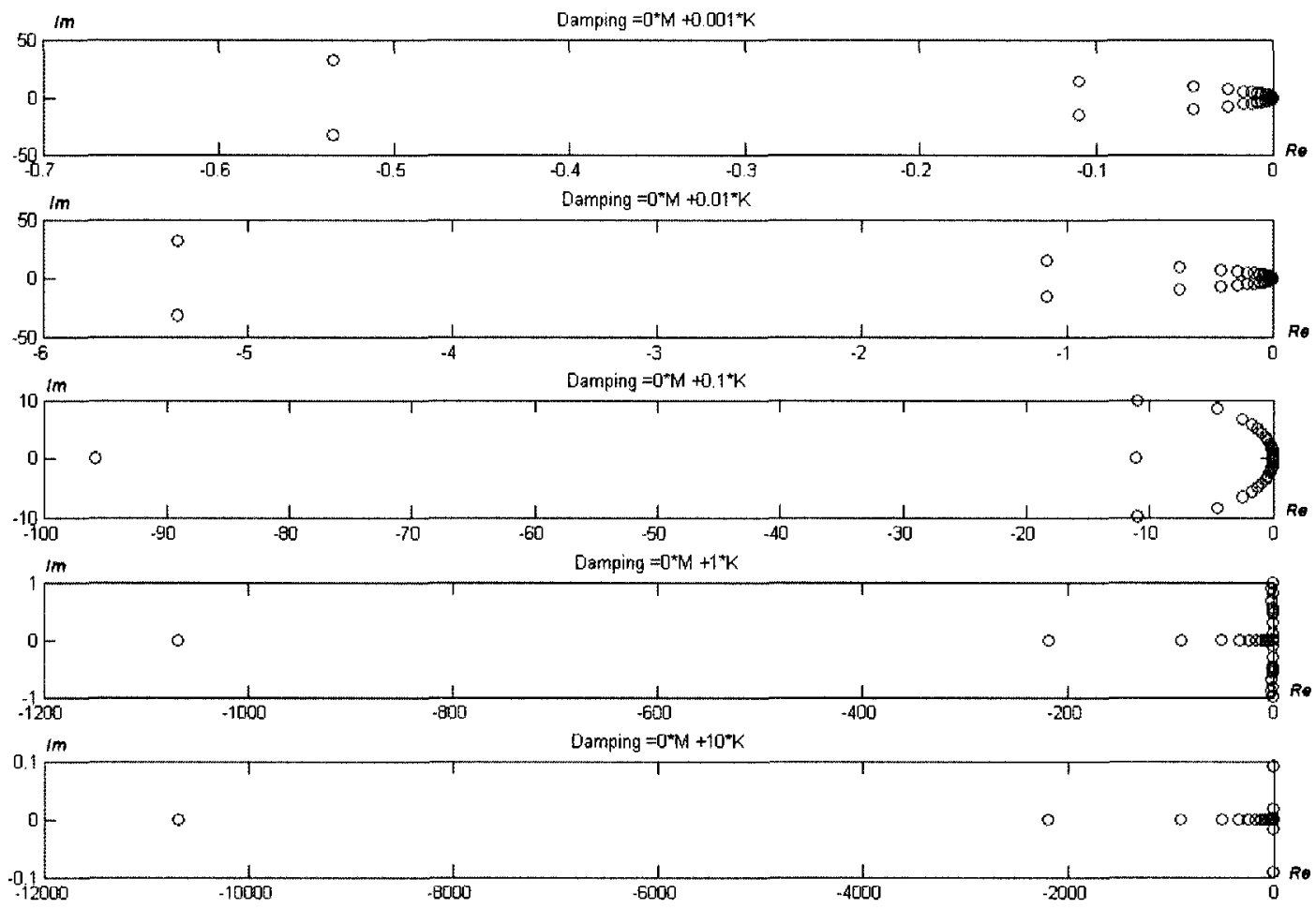

Figure E.1: Eigenvalues of Reduced Models using Rayleigh Damping (a) 

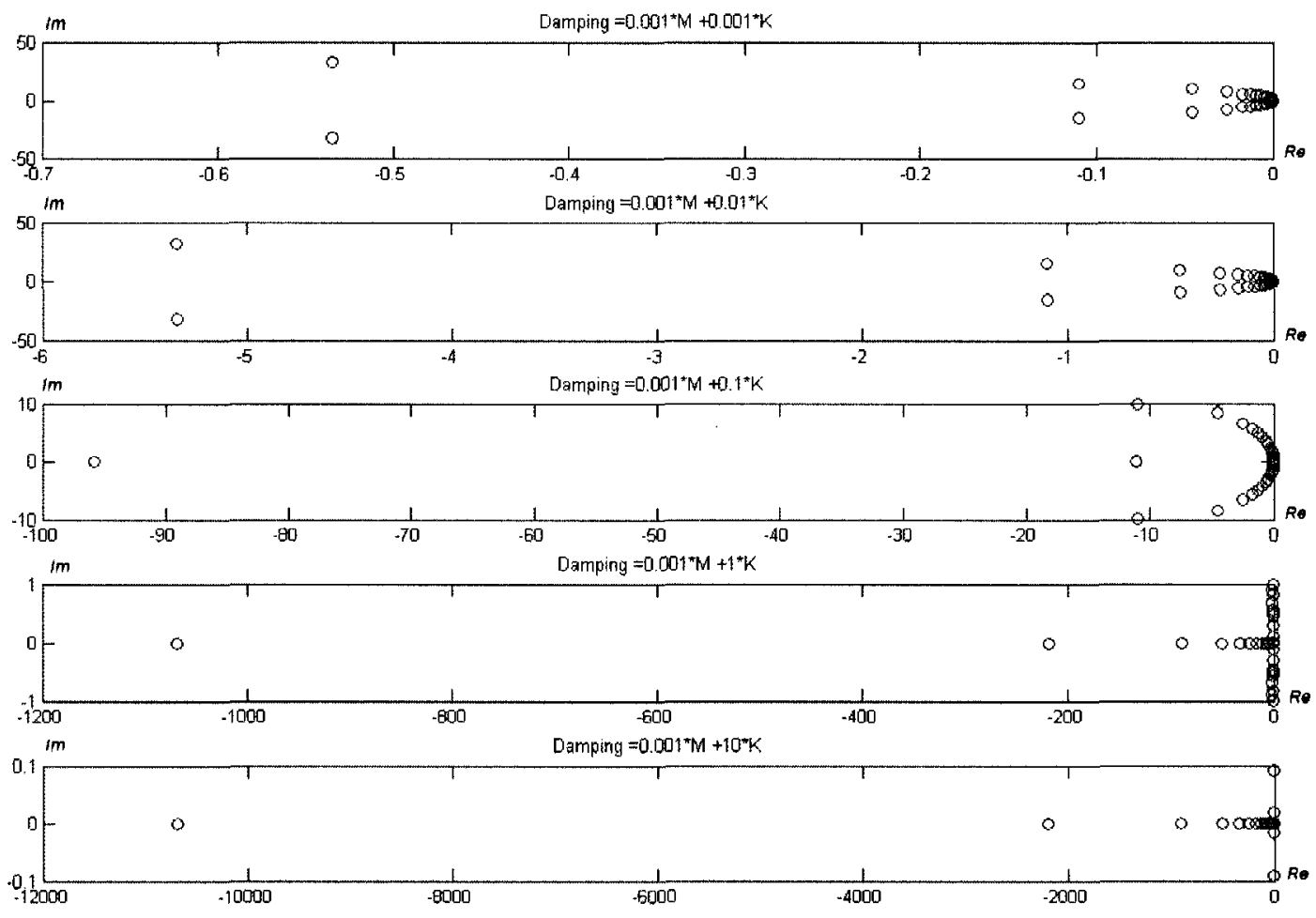

Figure E.2: Eigenvalues of Reduced Models using Rayleigh Damping (b) 

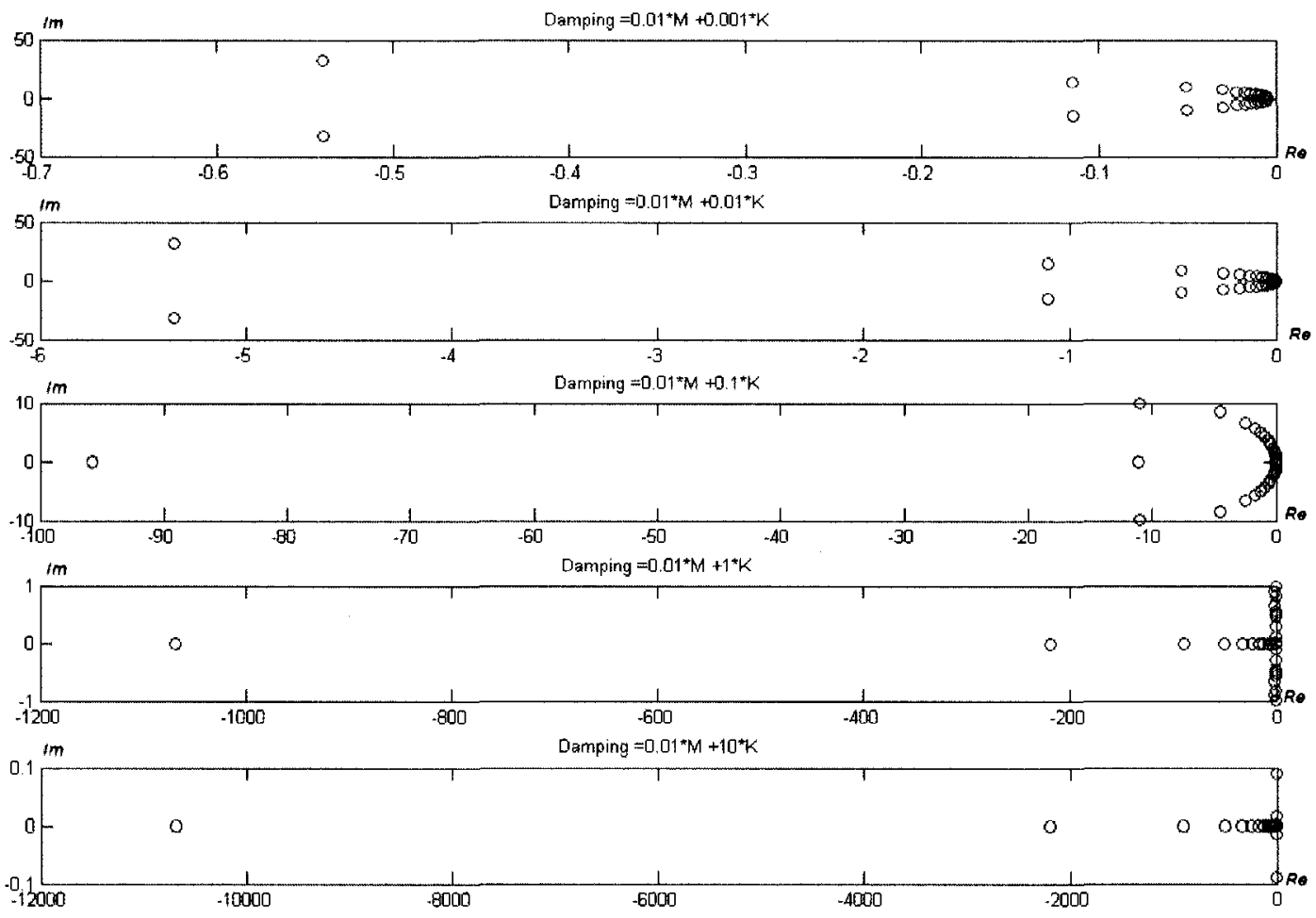

Figure E.3: Eigenvalues of Reduced Models using Rayleigh Damping (c) 

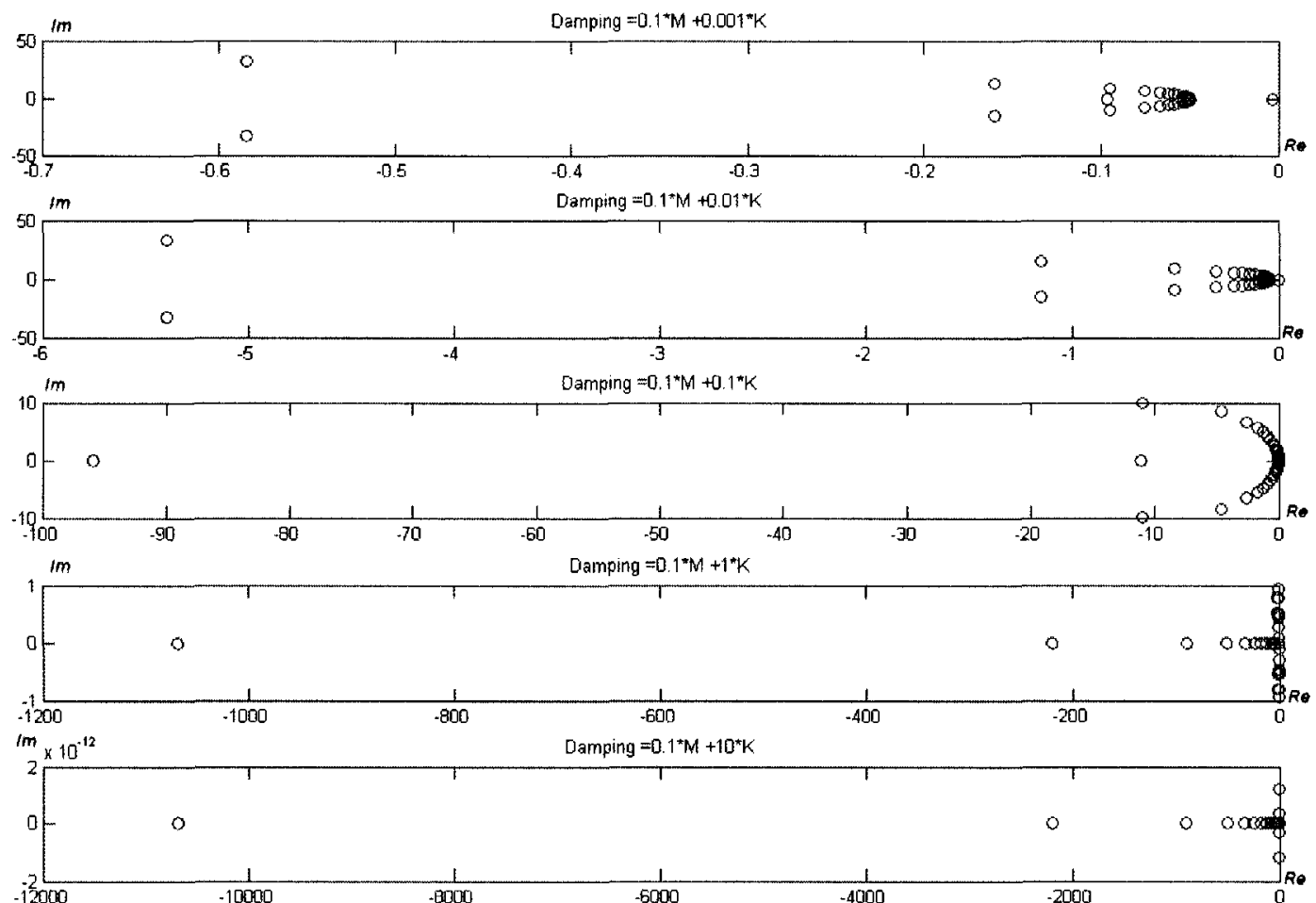

Figure E.4: Eigenvalues of Reduced Models using Rayleigh Damping (d) 


\section{References}

[1] J. Dongarra, Lecture Notes in Computer Science: Applied Parallel Computing. Springer Berlin/Heidelberg, 2006, vol. 3732.

[2] Y. Chahlaoui, D. Lemonnier, A. Vandendorpe, and P. V. Dooren, "Model Reduction of Second Order Systems," in Proceedings Fifteenth International Symposium on Mathematical Theory of Networks and Systems, 2002, pp. 1-15.

[3] "What is mems?" http://www.memsnet.org/.

[4] J. A. Pelesko and D. H. Bernstein, Modeling MEMS and NEMS. CRC Press, 2003.

[5] S. S. Rao, The Finite Element Method in Engineering. ButterWorth Heinemann, 1999.

[6] J. Clark, N. Zhou, and K. Pister, "MEMS simulation using SUGAR v0.5," In Technical Digest. Solid-State Sensor Workshop, pp. 191-196, 1998.

[7] Z. Bai, D. Bindel, J. V. Clark, J. Demmel, K. S. J. Pister, and N. Zhou, "New Numerical Techniques and Tools in Sugar for 3D MEMS Simulation," in Technical Proceedings of the Fourth International Conference on Modeling and Simulation of Microsystems, Hilton Head Island, SC, 2001, pp. 31-34.

[8] J. V. Clark, N. Zhou, D. Bindel, L. Schenato, W. Wu, J. Demmel, and K. S. J. Pister, "3D MEMS Simulation Modeling Using Modified Nodal Analysis," in Proceedings of the Microscale Systems: Mechanics and Measurements Symposium, Orlando, FL, 2000, pp. $68-75$.

[9] J. V. Clark, N. Zhou, and K. S. J. Pister, "Modified Nodal Analysis for MEMS with MultiEnergy Domains," International Conference on Modeling and Simulation of Microsystems, Semiconductors, Sensors and Actuators, pp. 31-34, 2000.

[10] E. Witmer, "Elementary Bernoulli-Euler Beam Theory," MIT Unified Engineering Course Notes: pp. 5-114 to 5-164, 1991-1992.

[11] C. W. D. Silva, Vibration: Fundamentals And Practice. CRC Press, 2006.

[12] I. Chowdhury and S. P. Dasgupta, "Computation of Rayleigh Damping Coefficients for Large Systems," The Electronic Journal of Geotechnical Engineering, www.ejge.com/2003/Ppr0318/Ppr0318.pdf, 2003. 
[13] L. Dumitriu and M. Iordache, "Order Reduction by Explicit Moment Matching Based on State Variable Approach," International Symposium on Signals, Circuits and Systems, 2007. ISSCS 2007, vol. 2, pp. 1-4, July 2007.

[14] P. K. Chan, "Comments on Asymptotic Waveform Evaluation for timing analysis," IEEE Transactions on Computer-Aided Design, pp. 1078-1079, August 1991.

[15] V. Balakrishnan, Q. Su, and C.-K. Koh, "Efficient Balance-and-Truncate Model Reduction for Large Scale Systems," in Proceedings of the American Control Conference, Arlington, VA, 2001, pp. 4746-4751.

[16] —, "Efficient Balance-and-Truncate Model Reduction for Large Scale Systems," in Model Reduction Using Balance-Truncation Method with Lower Error Bounds, vol. 2, Monterey, CA, USA, May 1991, pp. 934-938.

[17] R. Freund, "Reduced-order Modeling Techniques based on Krylov Subspaces and their use in Circuit Simulation," Bell Laboratories, Murray Hill, New Jersey, Tech. Rep., February 1998.

[18] B. Salimbahrami, R. Eid, and B. Lohmann, "Model Reduction by Second Order Krylov Subspaces: Extensions, Stability and Proportional Damping," in Proceedings of the 2006 IEEE Conference on Computer Aided Control Systems Design, Munich, Germany, October 2006, pp. 2997-3002.

[19] — - "Order Reduction of Large Scale Second-Order Systems using Krylov Subspace Methods," Linear Algebra and its Applications, pp. 385-405, 2006.

[20] T.-J. Su and R. R. C. Jr., "Model Reduction and Control of Flexible Structures Using Krylov Vectors," Journal of Guidance, Control, and Dynamics, vol. 14, no. 2, pp. 260$267,1991$.

[21] A. Ramchandra and M. Nakhla, "Simulation of High-Speed Interconnects," in Proceedings of the IEEE, vol. 89, no. 5, 2001, pp. 693-728.

[22] J. Y. Lee, "Efficient Pole Zero Sensitivity Analysis Using Asymptotic Waveform Evaluation (AWE)," Carnegie Mellon University, Pittsburgh,PA, USA, Tech. Rep., 1990.

[23] Y. I. Ismail, "Efficient Model Order Reduction via Multi-Node Moment Matching," in International Conference on Computer Aided Design, San Jose, California, 2002, pp. 767774.

[24] _ - "DTT: Direct Truncation of the Transfer FunctionAn Alternative to Moment Matching for Tree Structured Interconnect," IEEE Transactions on Computer-Aided Design of Integrated Circuits and Systems, vol. 21, no. 2, pp. 131-144, 2002.

[25] L. T. Pillage, R. A. Rohrer, and C. Visweswaria, Electronic Circuit and System Simulation Methods. New York: McGraw-Hill, 1994. 
[26] L. T. Pillage and R. A. Rohrer, "Asymptotic waveform evaluation for timing analysis," in IEEE Transactions on Computer-Aided Design, vol. 9, April 1990, pp. 352-366.

[27] D. F. Anastasakis, N. Gopal, S. Y. Kim, and L. T. Pillage, "On the Stability of Approximations in Asymptotic Waveform Evaluation," in Design Automation Conference, June 1992, pp. 207-212.

[28] L. He and S. Tan, Advanced Model Order Reduction Techniques in VLSI Design. Cambridge University Press, 2007.

[29] K. J. Kerns and A. T. Yang, "Stable and Efficient Reduction of Large, Multiport RC Networks by Pole Analysis via Congruence Transformations," in Annual ACM IEEE Design Automation Conference, 1996, pp. 280-285.

[30] H. A. van der Vorst, Iterative Krylov Methods for Large Linear Systems. Cambridge Press, 2003.

[31] Y. Saad, Iterative Methods for Sparse Linear Systems. Norwood, Mass.: Society for Industrial and Applied Math, 2003.

[32] I. C. Ipsen and C. D. Meyer, "The Idea Behind Krylov Methods," American Mathematical Monthly, vol. 105, no. 10, pp. 889-899, December 1998.

[33] G. H. Golub and C. F. van Loan, Matrix Computations. Oxford, England: North Oxford Academic, 1986.

[34] W. Arnoldi, "The Principle of Minimized Iterations in the Solution of the Matrix Eigenvalue Problem," Quarterly Applied Mathematics, vol. 9, p. 1729, 1951.

[35] S. B. Salimbahrami, "Structure Preserving Order Reduction of Large Scale Second Order Models," Ph.D. dissertation, Technische Universitat Munchen, Munich, Germany, October 2005.

[36] Z. Bai and Y. Su, "Dimension Reduction of Large Scale Second-order Dynamical Systems via a Second-order Arnoldi Method," SIAM Journal on Scientific Computing, vol. 26, pp. $1692-1709,2005$.

[37] E. B. Rudyni and J. G. Korvink, "Automatic Model Reduction for Transient Simulation of MEMS-based Devices," Sensors Update, vol. 11, pp. 2-33, 2002.

[38] R. W. Freund, "Padé-Type Model Reduction of Second-Order and Higher-Order Linear Dynamical Systems," in Dimension Reduction of Large-Scale Systems, ser. Lecture Notes in Computational Science and Engineering, P. Benner, V. Mehrmann, and D. Sorensen, Eds., vol. 45. Springer-Verlag, Berlin/Heidelberg, Germany, 2005, pp. 191-223.

[39] Z. Bai, K. Meerbergen, and Y. Su, "Arnoldi Methods for Structure-Preserving Dimension Reduction of Second-order Dynamical Systems," Springer Lecture Notes in Computational Science and Engineering, vol. 45, pp. 173-189, 2005. 
[40] Z. Bai and Y. Su, "SOAR: A Second-Order Arnoldi Method for the Solution of the Quadratic Eigenvalue Problem," SIAM Journal on Matrix Analysis and Applications, vol. 26, no. 3, pp. 640-659, 2005.

[41] D. S. Bindel, Z. Bai, and J. W. Demmel, "Model reduction for RF MEMS simulation," Applied Parallel Computing. State of the Art in Scientific Computing. 7th International Workshop, vol. 3732, pp. 286-295, 2004.

[42] J. Lampe and H. Voss, "Second Order Arnoldi Reduction: Application to Some Engineering Problems," Hamburg University of Technology, Tech. Rep., 2005.

[43] Z. Bai, "Krylov Subspace Techniques for Reduced-order Modeling of Large-Scale Dynamical Systems," Applied Numerical Mathematics, vol. 43, pp. 9-44, 2002.

[44] L. M. Silveira, M. Kamon, I. Elfadel, and J. White, "Coordinate-transformed Arnoldi algorithm for generating guaranteed stable reduced-order models of RLC circuits," IEEE/ACM International Conference on Computer-Aided Design, Digest of Technical Papers, pp. 288-294, 1996.

[45] J. Philips, L. Daniel, and L. M. Silveira, "Guaranteed Passive Balancing Transformations for Model Order Reduction," in Proceedings of the 39th Design Automation Conference, San Jose, 2002, pp. 52-57.

[46] I. Elfadel, L. Silveira, and J. White, "Stability Criteria for Arnoldi-Based Model-Order Reduction," in Acoustics, Speech, and Signal Processing, 1996. ICASSP-96 Conference Proceedings, vol. 5, May 1996, pp. 2642-2645.

[47] I. M. Elfadel and D. D. Ling, "Zeros and Passivity of Arnoldi-Reduced-Order Models for Interconnect Networks," in Design Automation Conference, 1997, pp. 28-33.

[48] R. W. Freund, "Krylov-subspace Methods for Reduced-order Modeling in Circuit Simulation," Journal of Computational and Applied Mathematics, vol. 123, pp. 395-421, November 2000.

[49] _ - "Reduced-order Modeling Techniques Based on Krylov Subspaces and their use in Circuit Simulation," in Applied and Computational Control, Signals, and Circuits, Boston, 1999, vol. 1, pp. 435-498.

[50] R. Li, “Test positive realness of a general transfer function matrix," Department of Mathematics, University of Kentucky, Lexington, KY, Tech. Rep., 2000.

[51] R. Freund, "Passive Reduced-Order Modeling via Krylov-Subspace Methods," in Proceedings of the 2000 IEEE International Symposium on Computer-Aided Control System Design, Anchorage, Alaska, 2000, pp. 261-266.

[52] J. Bastian and J. Haase, "Order Reduction For Second Order Systems," in Proceedings 4th MATHMOD, Vienna, Austria, 2003, pp. 418-424. 
[53] Z. Bai and R. Freund, "Eigenvalue-based Characterization and Test for Positive Realness of Scalar Transfer Functions," IEEE Transaction on Automatic Control, vol. 45, pp. 2396 $-2402,2000$.

[54] L. Knockaert, "A Note On Strict Passivity," Systems and Control Letters, vol. 54, pp. 865-869, 2005.

[55] N. Wong and C. Chu, "A Fast Passivity Test for Descriptor Systems Via StructurePreserving Transformations of Skew-Hamiltonian/Hamiltonian Matrix Pencils," 43rd ACM/IEEE Design Automation Conference, pp. 261-266, 2006.

[56] R. Freund and F. Jarre, "An Extension of the Positive Real Lemma to Descriptor Systems," Optimization Methods and Software, vol. 19, pp. 69-87, February 2004.

[57] Z. Bai and Y. Su, "Dimension Reduction of Second-Order Dynamical Systems via a Second-Order Arnoldi Method," SIAM Journal of Scientific Computing, vol. 26, no. 5, pp. 1692-1709, 2005.

[58] T. Stykel, "Balanced Truncation Model Reduction of Second-Order Systems," in Proceedings 5th MATHMOD, Vienna, Austria, 2006, pp. 1-9.

[59] P. Benner and D. Chu, "A New Test for Passivity of Descriptor Systems," in Oberwolfach Reports, vol. 2, no. 1, 2005, pp. 1-3.

[60] E. B. Rudyni, J. Lienemann, A. Greiner, and J. G. Korvink, "MOR4ANSYS: Generating Compact Models Directly from ANSYS models," in Technical Proceedings of the 2004 Nanotechnology Conference and Trade Show, Nanotech 2004, vol. 2, Bosten, Massachusetts, USA, 2004, pp. 279-282.

[61] J. S. Han, E. B. Rudyni, and J. G. Korvink, "Efficient Optimization of Transient Dynamic Problems in MEMS devices using Model Order Reduction," Journal of Micromechanics and Microengneering, vol. 15, pp. 822-832, 2005.

[62] M. Lehner and P. Eberhard, "A two-step Approach for Model Reduction in Flexible Multibody Dynamics," Multibody System Dynamics, vol. 17, pp. 157-176, 2007.

[63] A. Odabasioglu, M. Celik, and L. Pileggi, "PRIMA: Passive Reduced-Order Interconnect Macromodeling Algorithm," IEEE Transactions on Computer-Aided Design of Integrated Circuits and Systems, vol. 17, pp. 645-654, 1998.

[64] R. R. Craig and T. Su, "Krylov Model-Reduction Algorithm for Undamped Structural Dynamics," Journal of Guidance Control and Dynamics, vol. 14, pp. 1311-1313, 1991.

[65] H. J. D. L. Santos, "On the Ultimate Limits of IC Inductors An RF MEMS Perspective," in 52nd Proceedings of Electronic Components and Technology Conference, San Diego, CA, 2002, pp. 1027-1031. 
[66] E. Volterra and E. Zachmanoglou, Dynamics of Vibration. Columbus, Ohio: Charles E. Merrill Books Inc., 1965.

[67] W. T. Thomson, Theory of Vibration with Applications. Englewood Cliffs, New Jersey: Prentice Hall, 1972. 